\title{
Design and analysis of multilevel intervention studies
}

Citation for published version (APA):

Moerbeek, M. (2000). Design and analysis of multilevel intervention studies. [Doctoral Thesis, Maastricht University]. Universiteit Maastricht. https://doi.org/10.26481/dis.20000707mm

Document status and date:

Published: 01/01/2000

DOI:

10.26481/dis.20000707mm

Document Version:

Publisher's PDF, also known as Version of record

\section{Please check the document version of this publication:}

- A submitted manuscript is the version of the article upon submission and before peer-review. There can be important differences between the submitted version and the official published version of record.

People interested in the research are advised to contact the author for the final version of the publication, or visit the DOI to the publisher's website.

- The final author version and the galley proof are versions of the publication after peer review.

- The final published version features the final layout of the paper including the volume, issue and page numbers.

Link to publication

\footnotetext{
General rights rights.

- You may freely distribute the URL identifying the publication in the public portal. please follow below link for the End User Agreement:

www.umlib.nl/taverne-license

Take down policy

If you believe that this document breaches copyright please contact us at:

repository@maastrichtuniversity.nl

providing details and we will investigate your claim.
}

Copyright and moral rights for the publications made accessible in the public portal are retained by the authors and/or other copyright owners and it is a condition of accessing publications that users recognise and abide by the legal requirements associated with these

- Users may download and print one copy of any publication from the public portal for the purpose of private study or research.

- You may not further distribute the material or use it for any profit-making activity or commercial gain

If the publication is distributed under the terms of Article $25 \mathrm{fa}$ of the Dutch Copyright Act, indicated by the "Taverne" license above, 
Design and Analysis of Multilevel Intervention Studies 
(9) Mirjam Moerbeek, Maastricht 2000 ISBN 90-5681-074-X

Printing: Unigraphic, Maastricht University 


\section{Design and Analysis of Multilevel Intervention Studies}

\section{PROEFSCHRIFT}

ter verkrijging van de graad van doctor aan de Universiteit Maastricht, op gezag van de Rector Magnificus, Prof.dr. A.C. Nieuwenhuijzen Kruseman volgens het besluit van het College van Decanen, in het openbaar te verdedigen op vrijdag 7 juli 2000 om 14.00 uur

door

Mirjam Moerbeek

geboren op 13 februari 1973 te Westervoort 
Promotor:

Prof.dr. M.P.F. Berger

\section{Co-promotor:}

Dr. G.J.P. van Breukelen

\section{Beoordelingscommissie:}

Prof.dr. W.N.J. Groot (voorzitter)

Prof.dr.ir. P.A. van den Brandt

Dr. HJ.A. Schouten

Prof.dr. T.A.B. Snijders (Rijksuniversiteit Groningen)

Prof.dr. W.K. Wong (University of California at Los Angeles) 


\section{Acknowledgements}

This dissertation is the result of four years of work at the department of Methodology and Statistics of Maastricht University. The fact that my name is the only one appearing on the cover, does not mean that writing this dissertation was a solo performance. I have been fortunate to be surrounded by a number of people who supported me and gave critical but helpful comments on my research. First of all I wish to thank my supervisor Martijn Berger, not only for giving comments and advice on my research but also for creating a stimulating research environment by encouraging me to attend many scientific conferences and meetings. I thank my co-supervisor Gerard van Breukelen for his efforts he put into my research, for his detailed corrections of my papers and his help with the derivation of many formulae.

Martijn Berger encouraged me to spend a few months at the department of Biostatistics at the University of California at Los Angeles, where I worked together with Weng Kee Wong. I wish to thank him for allowing me to spend time at his department, for increasing my knowledge on optimal designs, and for his hospitality as well.

I wish to thank my colleagues Hubert Schouten and Frans Tan for our discussions on my research in particular and mixed effects models in general. Mario Ouwens worked on a PhD project closely related to mine, and all his questions not only helped both of us to progress further on our own respective papers. To any other colleagues who contributed to this thesis in one way or another: my sincere thanks!

I am very gratefull to my parents, for everything they have given me and have done for me, and to Martijn, whom I am glad to have as a brother. Writing a dissertation consumes an enormous amount of time. Luckily it did not take all my time, as I have been able to spend sufficient time socializing with friends.

Writing this thesis required much time and energy, and sometimes I wondered where it would all lead to, but $I$ have certainly leamed a lot these four years, and there is no doubt I would do it all over again if I would get another chance. 
a 


\section{Contents}

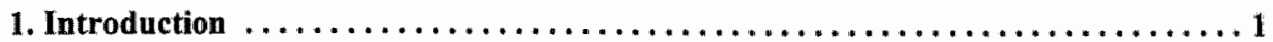

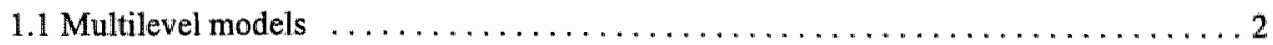

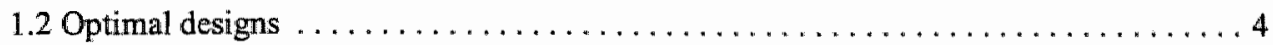

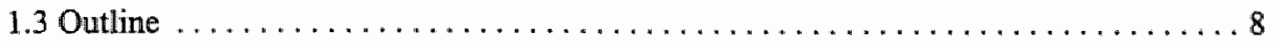

2. A review of four different methods for the analysis of multilevel experimental data $\mathbf{1 3}$

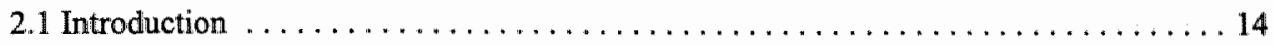

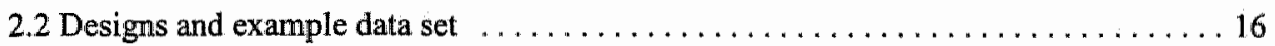

2.3 Multilevel regression model and mixed effects ANOVA model ............ 18

2.3.1 Design 1: Randomization at the pupil level $\ldots \ldots \ldots \ldots \ldots \ldots \ldots \ldots, 18$

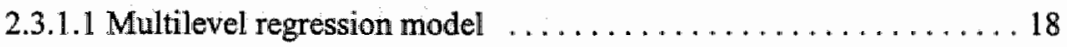

2.3.1.2 Mixed effects ANOVA model .......................... 20

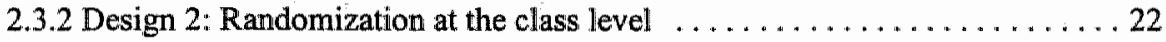

2.3.2.1 Multilevel regression model $\ldots \ldots \ldots \ldots \ldots \ldots \ldots \ldots \ldots 22$

2.3.2.2 Mixed effects ANOVA model $\ldots \ldots \ldots \ldots \ldots \ldots \ldots \ldots \ldots 22$

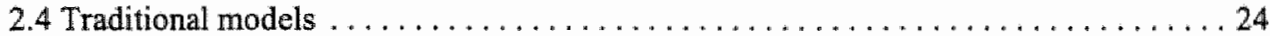

2.4.1 Fixed effects regression model and fixed effects ANOVA model ........ 24

2.4.2 Disaggregated data model and one-way ANOVA model $\ldots \ldots \ldots \ldots \ldots 25$

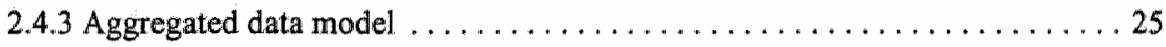

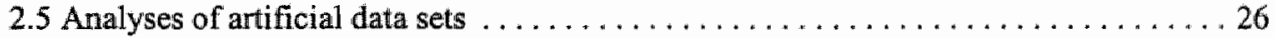

2.6 Comparison of the four methods based on analytical expressions $\ldots \ldots \ldots \ldots \ldots 27$

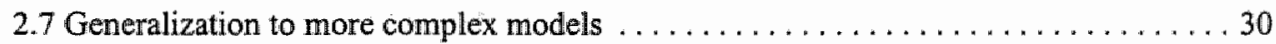

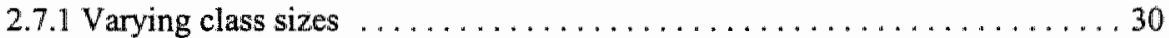

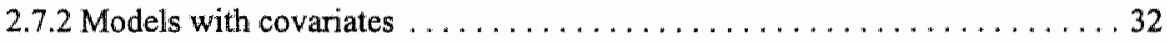

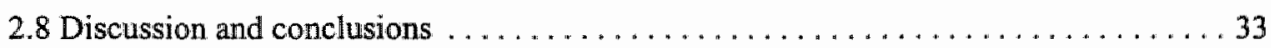


3. Optimal experimental designs for linear multilevel models $\ldots \ldots \ldots \ldots \ldots \ldots \ldots$

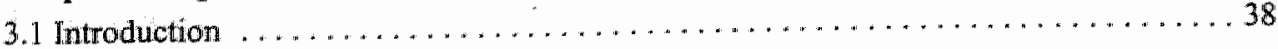

3.2 Models and estimators . . . . . . . . . . . . . . . . . . . . . . . . . . . . 39

3.3 Optimal experimental designs for the model with random intercept and fixed slope $\ldots 42$

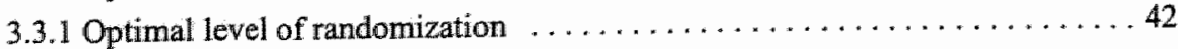

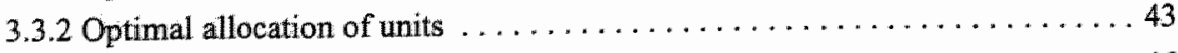

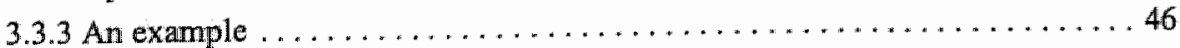

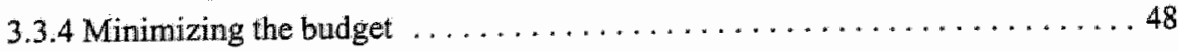

3.4 Optimal experimental designs for the model with random intercept and random slope . 49

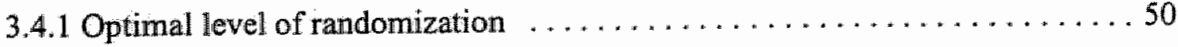

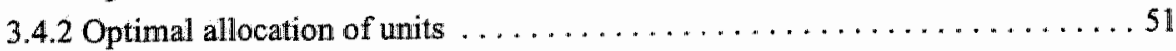

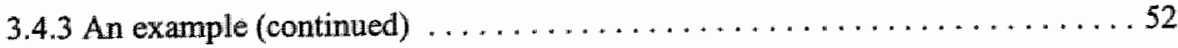

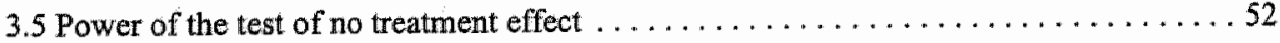

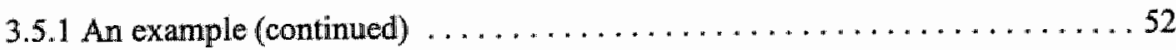

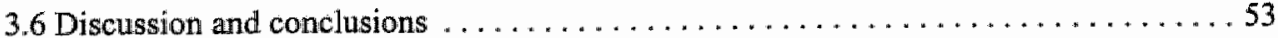

Appendix. Calculation of the $\operatorname{Var}\left(\beta_{1}\right)$ for known variance components $\ldots \ldots \ldots \ldots . \ldots 55$

4. Optimal experimental designs for linear multilevel models with covariates . . . . 57

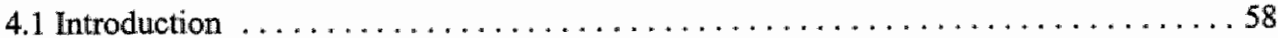

4.2 Models and estimators for a fixed treatment effect $\ldots \ldots \ldots \ldots \ldots \ldots \ldots \ldots \ldots . \ldots 59$

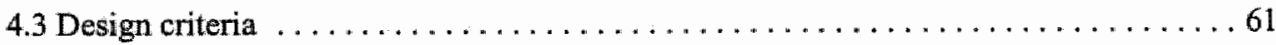

4.4 Optimal designs for models with a fixed treatment effect $\ldots \ldots \ldots \ldots \ldots \ldots \ldots \ldots$

4.5 Effects on the estimated variance components of excluding covariates from the model

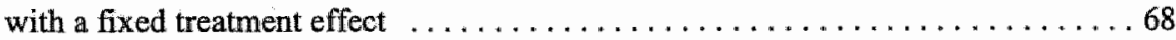

4.6 Optimal designs for models with a random treatment effect $\ldots \ldots \ldots \ldots \ldots \ldots \ldots$

4.7 Effects on the estimated variance components of excluding covariates from the model

with a random treatment effect $\ldots \ldots \ldots \ldots \ldots \ldots \ldots \ldots \ldots \ldots \ldots \ldots$

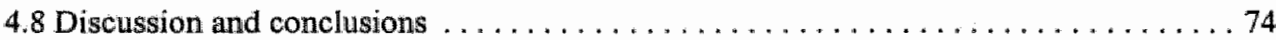




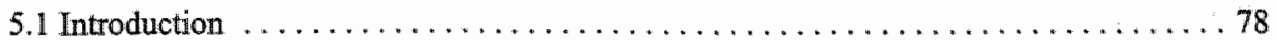

5.2 The multilevel logistic model and its estimation $\ldots \ldots \ldots \ldots \ldots \ldots \ldots \ldots$

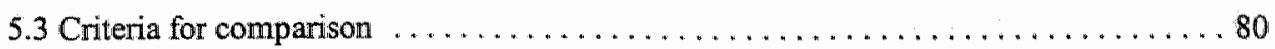

5.3 .1 Criteria concerning point estimation $\ldots \ldots \ldots \ldots \ldots \ldots \ldots \ldots \ldots$

5.3 .2 Criteria conceming testing of the treatment effect $\ldots \ldots \ldots \ldots \ldots \ldots$. . . 80

5.4 Convergence and behavior of the estimation procedure $\ldots \ldots \ldots \ldots \ldots \ldots \ldots \ldots$

5.5 Randomization at person level and assuming no treatment by cluster interaction $\ldots \ldots 82$

5.5 .1 Criteria concerning point estimation $\ldots \ldots \ldots \ldots \ldots \ldots \ldots \ldots \ldots$

5.5.2 Criteria concerning testing of the treatment effect $\ldots \ldots \ldots \ldots \ldots \ldots . \ldots 4$

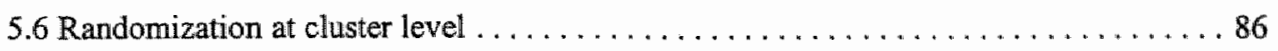

5.6 .1 Criteria concerning point estimation . . . . . . . . . . . . . . 86

5.6 .2 Criteria concerning testing of the treatment effect $\ldots \ldots \ldots \ldots \ldots \ldots$

5.7 Randomization at person level and assuming treatment by cluster interaction . . . . . 89

5.7.1 Criteria concerning point estimation . . . . . . . . . . . . . . 89

5.7.2 Criteria conceming testing of the treatment effect . . . . . . . . . . . 89

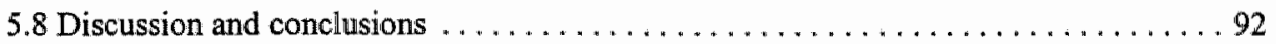

6. Optimal experimental designs for multilevel logistic models $\ldots \ldots \ldots \ldots \ldots \ldots$

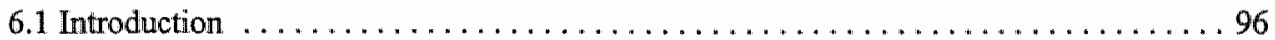

6.2 The multilevel logistic model and its estimation using Quasi Likelihood or numerical

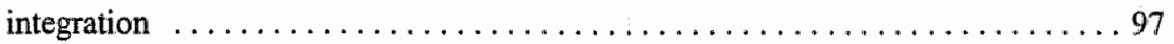

6.3 Optimal experimental designs for the model with random intercept and fixed slope . . . 99

6.3.1 Optimal experimental designs for first order $\mathrm{MQL}$ with known variance components ................................. 99

6.3.2 Optimal experimental designs for PQL and numerical integration . . . . . 102

6.3.3 An example: Planning of a school-based smoking prevention intervention . . 106

6.4 Optimal experimental designs for the model with random intercept and random slope 107

6.4.1 Optimal experimental designs for first order $\mathrm{MQL}$ with known variance components ................................... 107

6.4.2 Optimal experimental designs for PQL and numerical integration . . . . ... 108

6.4.3 An example: planning of a school-based smoking prevention intervention

(continued) . . . . . . . . . . . . . . . . . . . . . . . . . . . 109

6.5 Power of the test of no treatment effect $\ldots \ldots \ldots \ldots \ldots \ldots \ldots \ldots \ldots \ldots \ldots$

6.5.1 An example: planning of a school-based smoking prewention intervention

(continued) . ................................. 110

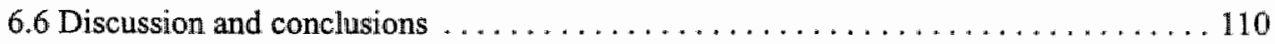

Appendix. Calculation of the $\operatorname{Var}\left(\beta_{1}\right)$ for known variance components $\ldots \ldots \ldots \ldots \ldots$ 
7. Optimal sample sizes for health education intervention studies

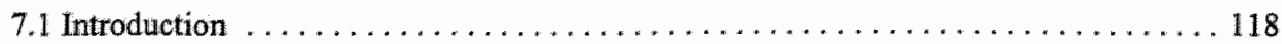

7.2 Analysis of the data from the Dutch smoking prevention intervention $\ldots \ldots \ldots 119$

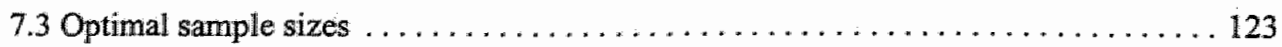

7.4 Optimal sample sizes for the Dutch smoking prevention intervention $\ldots \ldots \ldots 126$

7.4 .1 Optimal sample sizes for the outcome variable attitude $\ldots \ldots \ldots \ldots \ldots 127$

7.4.2 Optimal sample sizes for the outcome variable smoking behavior $\ldots \ldots \ldots 131$

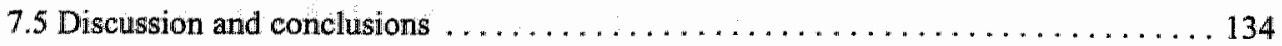

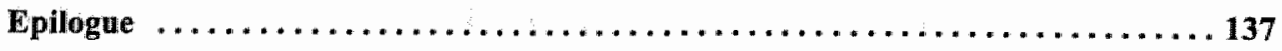

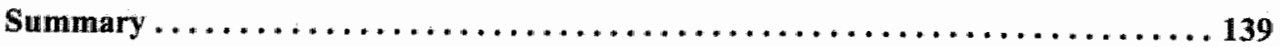

Samenvatting in het Nederlands (Dutch summary) $\ldots \ldots \ldots \ldots \ldots \ldots \ldots \ldots$

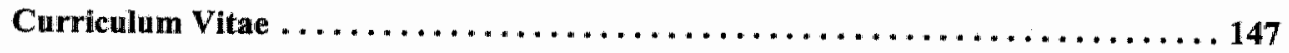




\section{Chapter 1}

\section{Introduction}

This thesis deals with the design and analysis of experiments in settings where individuals are nested within clusters, which may themselves be nested within higher order clusters. Examples are school-based smoking prevention interventions with pupills nested within classes within schools (e.g., Hedeker, Gibbons, and Flay, 1994), multi-center clinical trials with patients nested within clinics (Hedeker, Gibbons, and Davis, 1991), studies on absence due to illness with employees nested within companies, and studies on achievement with students nested within tutorial groups in problem-based learning. Such experiments generally demand a high amount of time, money, and labor, and the willingness of subjects to participate in the experiment is necessary. These efforts could be somewhat wasted if the experiment were not designed optimally. Different optimality criteria may be used to select such a design, and these criteria may lead to different optimal designs. Therefore, the optimality criterion has to be chosen carefully. The statistical model that will be used to analyze the data has to be chosen beforehand as well.

Traditionally, textbooks on the design and analysis of experiments mainly deal with the situation where outcomes of different individuals are assumed to be independent. This assumption, however, is not plausible in the situation where individuals are nested within clusters, since individuals within a cluster influence each others behavior and attitude by direct communication or shared group norms. Their behavior and attitude may also be influenced by, for example, cluster environment, cluster policy, or cluster leaders. Optimal designs for independent outcomes are not optimal for experiments in settings were individuals are nested within clusters, and the traditional regression model will lead to an incorrect data analysis, since they do not take into account the nesting of individuals within clusters and the dependency of outcomes within the same cluster. The general statistical model that is applicable to this kind of data is the multilevel regression model, which may be used when the clusters in the study are assumed to represent a random sample from some population. As will be shown in this thesis, multilevel models may lead to optimal designs and estimates of model parameters and 
corresponding standard errors that differ from those obtained by traditional statistical models, and this especially occurs when the dependency of outcomes within a cluster is large.

In the next section a short introduction to multilevel modeling will be given, as well as a brief historical sketch of multilevel models and their estimation. Thereafter the basic ideas of optimal designs will be given in Section 1.2. Finally, an overview and motivation of the contents of the chapters in this thesis will be presented.

\subsection{Multilevel models}

The terms multilevel model and hierarchical model are often used in educational sciences and sociology. In statistical literature, these kind of models are more often referred to as covariance components model and random coefficient model. The term random coefficient model is also common in econometrics, whereas the terms random effects models or mixed effects models are mostly used in biometrics. Mixed effect models contain both fixed and random effects. Fixed effects are effects that are attributable to a finite set of levels of a factor. These levels occur in the data since we are interested in only them, and not in any other levels of that factor. Random effects, on the other hand, are attributed to an infinite set of levels of a factor, of which only a random sample is included in the study at hand. The distinction between fixed, random, and mixed effects models seems to have been introduced by Eisenhart (1947).

Multilevel models for two levels of nesting will be presented in this section. The extension to more levels of nesting is straightforward. Individuals are the level-one or microlevel units, and clusters are the level-two or macro-level units. The basic idea of multilevel modeling is that regression coefficients may vary across clusters as a function of cluster level predictor variables and/or a random cluster effect. The model which relates the outcome $y_{i j}$ of person $i$ in cluster $j$ to an individual level predictor variable $x_{i j}$ is given by

$$
y_{i j}=\beta_{0 j}+\beta_{1 j} x_{i j}+e_{i i^{\prime}}
$$

where the random error term $e_{i j} \sim \mathrm{N}\left(0, \sigma_{e}^{2}\right)$ measures the discrepancy between the $y_{i j}$ as predicted from the $x_{i j}$ and the actual outcome $y_{w}$, and the regression coefficients $\beta_{0 j}$ and $\beta_{1 y}$ may vary across clusters so that each cluster may have its own regression equation. Model (1.1) is referred to as the individual level model since the variables $y_{i j}$ and $x_{i j}$ are both measured at that level. Suppose that both the intercept $\beta_{0 j}$ and slope $\beta_{1, j}$ vary as a function of a cluster level predictor variable $z_{j}$ and a random cluster effect, then we have the cluster level model

$$
\begin{aligned}
& \beta_{0 j}=\beta_{00}+\beta_{01} z_{j}+u_{0 j} \\
& \beta_{1 j}=\beta_{10}+\beta_{11} z_{j}+u_{1 j}
\end{aligned}
$$

where the random terms at the cluster level $u_{0 j} \sim \mathrm{N}\left(0, \sigma_{w 0}^{2}\right)$ and $u_{1 j} \sim \mathrm{N}\left(0, \sigma_{u 1}^{2}\right)$ may be correlated, 
and their covariance is denoted $\sigma_{w 01}$. The single equation model is obtained by substitution of (1.2) into (1.1):

$$
y_{i j}=\left(\beta_{00}+\beta_{01} z_{j}+\beta_{10} x_{i j}+\beta_{11} x_{i j} z_{j}\right)+\left(u_{0 j}+u_{i j} x_{i j}+e_{i j}\right)
$$

where the part between the first pair of brackets is referred to as the fixed part, and the part between the second pair of brackets is called the random part. The term $x_{i j} z_{j}$ is a cross-level interaction term since it is a product of predictor variables at level-one and level-two. Model (1.3) may be extended or simplified by including or excluding more individual and cluster level predictor variables and/or random terms. If necessary, more levels of nesting may be included as well.

Model (1.3) is an extension to the traditional regression model since it not only contains an individual level error term, but also random terms at the cluster level. As a consequence, the variance of the outcome for individual $i$ within cluster $j$ given the predictor variables is equal to

$$
\operatorname{Var}\left(y_{i j}\right)=\sigma_{w 0}^{2}+x_{i j}^{2} \sigma_{u 1}^{2}+2 x_{i j} \sigma_{u 01}+\sigma_{i}^{2}
$$

and so there is heteroscedasticity in the variances. Moreover, there is also correlation between outcomes of individuals $i$ and $i$ ' within the same cluster $j$ :

$$
\operatorname{Cov}\left(y_{i j^{\prime}}, y_{i, j}\right)=\sigma_{u 0^{2}}^{2}+x_{i j^{\prime}} x_{i j} \sigma_{u 1}^{2}+\left(x_{i j}+x_{i j}\right) \sigma_{u 01}
$$

So the ordinary least squares estimatior, which assumes homoscedasticity and uncorrelated outcomes, should not be used for the analysis of model (1 .3) since it will lead to incorrect results.

Although clustered data structures have since long been recognized, the estimation of mixed effects and random effects models was a difficulty because of the lack of suitable estimation methods and computer programs. For a long time the analysis of variance models, like the split-plot design madel, were the only models for the analysis of clustered data. Analysis of variance models generally work well for continuous outcomes, categorical predictor variables, and balanced designs. Estimation methods for unbalanced data were already developed by Henderson (1953), but these were restricted to categorical predictor variables. For models with continuous predictor variables no useful models and estimation methods were available at that time, and alternative models like the aggregated data or summary measures model, the disaggregated data model, or the fixed effects regression model were used instead. The aggregated data model aggregates data to the cluster level; and clusters are the unit of analysis. The disaggregated data model treats individuals as the unit of analysis, and their nesting within clusters and dependency of outcomes within the same cluster is ignored. The fixed effects model treats clusters as fixed and their differences are taken into account by dummy coding in the regression model. 
A general method for estimation of mixed and random effects models became available with the development of Maximum Likelihood (ML; Hartley and Rao, 1967), Restricted Maximum Likelihood estimation (REML; Patterson and Thompson, 1971), and Minimum Norm Quadratic Unbiased Estimation (MDNQUE; LaMotte, 1973; Rao, 1970, 1971a, b, 1972). Bayes estimates for multilevel models were presented by Lindley and Smith (1972). During the 1980s much research was done on the development of methods for the computation of ML and REML estimates for linear multilevel models, which resulted in (Iterative) Generalized Least Squares (IGLS and RIGLS; Goldstein, 1986, 1989), and the application of already existing methods like the Fisher scoring algorithm (Longford, 1987) and the EM algorithm (Dempster, Rubin, and Tsutakawa, 1981; Mason, Wong, and Entwisle, 1984). Estimation methods for non-linear multilevel models were developed during the 1990s, based on Quasi Likelihood methods (Goldstein, 1991; Goldstein and Rasbash, 1996) and on numerical integration of the likelihood function (Gíbbons and Hedeker, 1997; Hedeker and Gibbons, 1994).

Nowadays, estimation of multilevel models can be done by means of software packages like MLwiN (Goldstein et at., 1998), HLM (Bryk, Raudenbush, and Congdon, 1996), and MIXOR/MLXREG (Hedeker and Gibbons, 1996a, 1996b). Introductory and more advanced textbooks on multilevel analysis were written by Bryk and Raudenbush (1992), Goldstein (1995), Hox (1994), Kreft and De Leeuw (1998), Longford (1995), and Snijders and Bosker (1999).

The multilevel model may be used for the analysis of different kinds of clustered data. In this dissertation we will restrict ourselves to multilevel models for experimental data in a hierarchically structured population, such as school-based smoking prevention interventions with pupils nested within schools. In these kinds of studies the randomization to treatment conditions is under experimental control, which is not the case for covariates such as age and gender. Multilevel models may also be used for the analysis of observational studies where the data are not under experimental control, such as that obtained from school effectiveness studies (e.g., Aitkin and Longford, 1986) where pupils are nested within classes within schools. The last application of multilevel models to mention here is that to longitudinal data with repeated measurements nested within persons (e.g. Laird and Ware, 1982; Snijders, 1996).

\subsection{Optimal designs}

Choosing an optimal design means to choose a design that satisfies a statistical optimality criterion, given some pre-conditions. Examples of such preconditions are the precondition that the costs of the experiment may not exceed an available budget, and the precondition that the sample sizes at each level of the multilevel data structure have to be positive. Sometimes not all combinations of the levels of the treatment factors are feasible, and experiments are often limited by political or ethical considerations, the need to reduce administrative efforts, and the need to avoid treatment group contamination, which occurs when information leaks from the intervention group to the control group. 
Crucial to the planning of an optimal design is the choice of the analysis madel, since different models may lead to different optimal designs. In this section we will assume that the multilevel model in (1.3) is the correct analysis model, optimal design construction for other models is likewise. For the model (1.3) the parameter vector $\theta$ contains both fixed terms and variances and covariances of the random terms: $\theta=\left(\beta_{00,}, \beta_{01}, \beta_{10 *}, \beta_{11}, \sigma_{e}^{2}, \sigma_{w 0}^{2}, \sigma_{w 1}^{2}, \sigma_{w 01}\right)^{\prime}=$ $\left(\beta^{*}, \sigma^{2}\right)^{\prime}$. Information on these parameters is provided by the data, and may be captured by the Fisher information matrix $M(X, \theta)$. This matrix is defined as minus the expectation of the second derivative of the logarithm of the likelihood function $L(X, \theta)$ :

$$
\boldsymbol{M}(\boldsymbol{X}, \theta)=-E\left(\frac{\partial^{2} \log L\left(\boldsymbol{X}_{\mathrm{s}} \theta\right)}{\partial \theta \partial \theta^{\prime}}\right),
$$

where $\log L=-\frac{1}{2} \sum_{j} n_{1 j} \log 2 \pi-\frac{1}{2} \log \mid V-\frac{1}{2}(y-X \beta)^{\prime} V^{-1}(y-X \beta)$ and $n_{y}$ is the number of individuals in cluster $j$. The design matrix $\boldsymbol{X}$ contains the measures on the predictor variables, its row subscribed $i j$ corresponds to the predictor variables of individual $i$ in cluster $j$ and is equal to $\left(\begin{array}{llll}1 & z_{j} & x_{i j} & z_{j} x_{i j}\end{array}\right)$. The matrix $V$ is the covariance matrix of the outcome vector $y$. The Fisher information matrix contains information on each parameter and each combination of two parameters. Asymptotically it is equal to the inverse of the covariance matrix of the estimated parameters, which for model (1.3) is equal to

$$
M^{-1}(X, \theta)=\left(\begin{array}{ll}
M_{11}(X, \theta) & M_{12}(X, \theta) \\
M_{21}(X, \theta) & M_{22}(X, \theta)
\end{array}\right)^{-1}
$$

where $M_{11}^{1}(X, \theta)=\operatorname{Cov}(\hat{\beta}), M_{22}^{1}(X, \theta)=\operatorname{Cov}\left(\theta^{2}\right)$, and $M_{12}(X, \theta)=M_{21}(X, \theta)=0$ since $\operatorname{Cov}\left(\beta, \theta^{2}\right)=0$ for the linear model (1.3). The non-zero submatrices of $M(X, \theta)$ are equal to

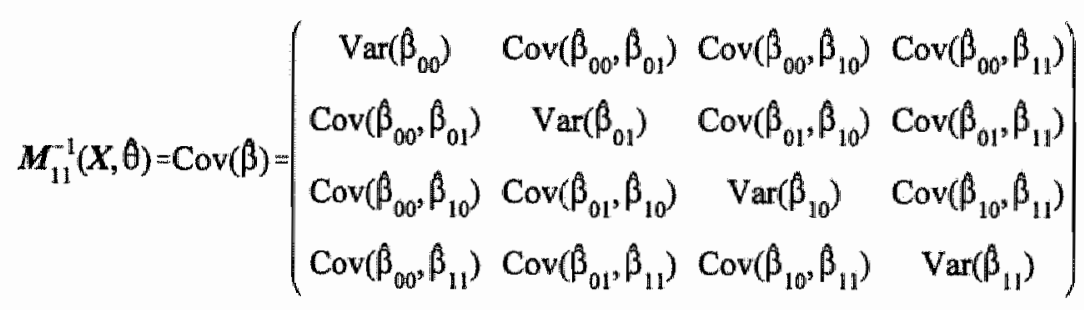

and 


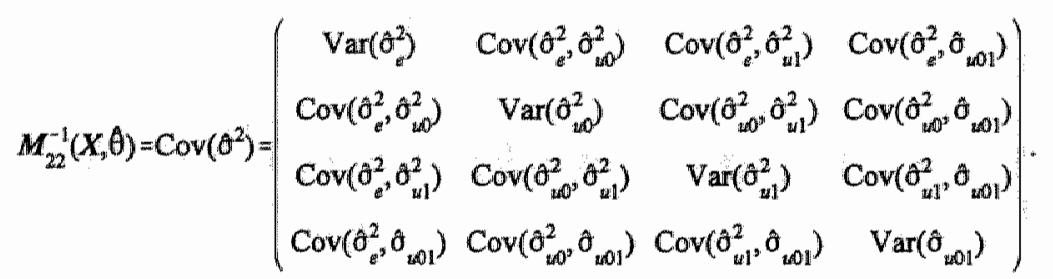

In the above the Fisher information matrix was written as $M(X, \theta)$ to stress that it is a function of the design matrix $X$, and the parameter vector $\theta$. In their turn, the elements of the design matrix $\boldsymbol{X}$ depend on the chosen regression model $\eta$, and on the design $\xi$, which provides information on the level of randomization and the number of individuals per treatment per cluster. To stress the dependency of the Fisher information matrix on the parameter vector, and on the design matrix, which in its turn depends on the model and the design, we will use the notation $M(X(\xi, \eta), \theta)$ in the remainder of this chapter, instead of the short notation $M(\xi)$, which is more common in optimal design literature.

Choosing an optimal design means to choose the design $\xi^{*}$ among all the designs $\xi$ in the design space $\chi$ that provides maximum information on the parameters that have to be estimated. So the Fisher information matrix $\boldsymbol{M}(\boldsymbol{X}(\xi, \eta), \theta)$ must be maximized in some way, and this is equivalent to minimizing its inverse $\boldsymbol{M}^{-1}(X(\xi, \eta), \theta)$. In this thesis we will restrict ourselves to the fixed parameters $\beta$, so $\boldsymbol{M}_{11}^{-1}(\boldsymbol{X}(\xi, \eta), \theta)$ has to be minimized. Since matrices cannot be ordered in a unique way, various functions have been proposed as optimality criteria, which at least have to be convex and differentiable. Depending on the research question, various functions $\Psi$ of the matrix $\boldsymbol{M}_{11}^{-1}(\boldsymbol{X}(\xi, \eta), \theta)$ are available. If, for example, interest only lies in the parameter $\beta_{k}$, one should minimize its corresponding variance:

$$
\text { minimize } \operatorname{Var}\left(\hat{\beta}_{k}\right) \text {. }
$$

As a result its confidence interval will be as narrow as possible, and the power of the corresponding test will be as high as possible. On the other hand, such a design may be far from optimal for the estimation of other parameters.

A more general optimality criterion is the $D$ - optimality criterion which minimizes the generalized variance of the parameter estimates:

$$
\text { minimize } \log \left|M_{11}^{-1}(X(\xi, \eta), \theta)\right| \text {. }
$$

The logarithm of the determinant is taken because this ensures numerical stability of the convex function. The $D$ - optimality criterion minimizes the wolume of the confidence ellipsoid of the parameters, and takes into account the variances and covariances of the parameter estimators simultaneously. A very long and thin ellipsoid that is oriented along or close to the parameter 
axes results in a poor estimate of one or more of the parameters since their corresponding variances are large.

The $D$ - optimality criterion takes into account both variances and covariances of parameter estimates. When interest only lies in the variances, the $A$ - optimality criterion may be more appropriate:

$$
\text { minimize } \operatorname{trace}\left(M_{11}^{1 !}(X(\xi, \eta), \theta)\right)
$$

The $A$ - optimality criterion minimizes the sum of the variances of the parameter estimators.

The last criterion to mention here is that of $c$ - optimality, which minimizes the variance of a linear combination $c^{\prime} \beta$ of the regression coefficients:

$$
\text { minimize } c^{\prime} M_{11}^{-1}(X(\xi, \eta), \theta) c
$$

Of course, the optimal designs obtained with this criterion strongly depend on the choice of the vector $c$. Note that the optimality criterion in (1.10) is a special case of the $c$ - optimality criterion, when the $(k+1)$-th element of the vector $c$ is equal to one, whereas the other elements are set equal to zero. This optimality criterion may also be used for contrasts. The $D-, A-$, and $c$-optimality criteria are most easily interpreted when all predictor variables are measured in the same unit. For other optimality criteria see, for example, Silvey (1980), and Atkinson and Donev (1996).

The optimal design $\xi$ may not always be feasible in practice. Then an alternative design $\xi$ may be chosen, and the efficiency of its corresponding design relative to the optimal design is equal to

$$
\text { relative efficiency }=\frac{\Psi\left(M_{11}^{-1}\left(X\left(\xi^{*}, \eta\right), \theta\right)\right)}{\Psi\left(M_{11}^{-1}(X(\xi, \eta), \theta)\right)}
$$

for $A$-, and $c$-optimality, and this ratio is always between zero and one. Its inverse gives the number of times the design $\xi$ needs to be replicated to be as efficient as the optimal design $\xi^{*}$. To maintain this nice interpretation, the $p$-th root of the right-hand side of (1.14) has to be taken for $D$ - optimality, where $p$ is the number of regression coefficients $\beta$. Ideally the relative efficiency of a design $\xi$ is close to one.

Optimal designs for non-linear models may be derived by linearizing the non-linear part of the model using a Taylor series expansion. The optimality criterion may then be applied to the linearized model. Of course, a higher order for the expansion leads to a better approximation to the optimal design, but also to more computational efforts. For mixed-effects models the linearization may be done around the fixed part only, or around both the fixed and random part. The first method may lead to seriously biased estimates, but for the latter method, analytical 
expressions for the optimal design cannot be obtained and a simulation study is needed to obtain the optimal design.

In this thesis optimal designs will be derived for linear and logistic multilevel models with a treatment factor having two levels. The treatment effect estimator is of main interest and its variance will be minimized to obtained smallest possible confidence intervals and highest power of the test on treatment effect. This optimality criterion is applied in Chapters 3,4, and 6. Other optimality criteria will be applied as well in Chapter 4. The level of randomization and allocation of units that minimize the optimality criterion will be established. It has to be noted that intervention studies often have different outcome variables. A design which is optimal for a certain outcome variable may be less optimal for other outcome variables, and therefore designs which are acceptable for all outcome variables of interest have to be chosen.

\subsection{Outline}

In this dissertation some contributions are given to the design of experiments for the situation where individuals are nested within clusters, and to the analysis of data obtained from such experiments. This thesis mainly contributes to stages $2 \mathrm{e}$ and 4 of the stages which are part of the experimental or empirical cycle:

\section{Pre-study}

\section{Design stage}

a. choice of interventions

b. choice of covariates

c. choice of outcome variables and analysis model

d. practical restrictions and limitations

e. optimality criterion and optimal design

3. Conduct of the experiment

4. Analysis and results

5. Suggestions for future research

The chapters in this thesis may be read as self-contained papers. The objectives and motivation of the chapters are as follows:

Chapter 2. The multilevel model is more complicated than traditional statistical models, and special software is needed when using such models. Therefore many applied researchers still use traditional models like the fixed effects model, the disaggregated data model, and the aggregated data model. A comparison is made in this chapter to show when and how these traditional models fail, and to convince applied researchers that traditional models are generally not a good alternative to the multilevel model. The comparison is made for experiments with continuous outcomes and two levels of nesting, and where randomization is done either at the individual level or at the cluster level. 
Chapter 3. Three questions that arise when planning experiments are introduced in this chapter. These issues will also be addressed in Chapters 4 and 6 in which more complicated models are used. The first question concerns the optimal level of randomization. Interventions are often randomized at the cluster level and one might wonder to what extent randomization at a lower level, if possible, may lead to a more efficient design. The second question concerns the optimal sample sizes. Many researchers calculate the optimal sample size ignoring the nesting of individuals within clusters. This may lead to sample sizes that are far from optimal. In fact, not only the optimal total number of individuals has to be established, but also the distribution of individuals across clusters. Do we need to sample many clusters with just a few individuals, or vice versa? The third question deals with the power of the test on treatment effect that may be obtained with a chosen design. In this chapter these questions will be addressed for models with continuous outcomes, without covariates, and two or three levels of nesting. The variance of the treatment effect estimator will be used as optimality criterion since this parameter is often of main interest in experiments. Sample sizes are often limited by financial supports, and this will be used as a precondition for calculating the optimal design.

Chapter 4. Often covariates are included into the model for several purposes, among. others to increase power by reducing unexplained variance, and in quasi-experiments also to adjust for group non-equivalence. Chapter 4 builds upon the results in Chapter 3 by considering models in which covariates at both the individual and cluster level may be included into the multilevel model. These covariates may or may not correlate with the treatment effect. Several optimality criteria will be used for establishing the optimal design. Also the effect of the inclusion or exclusion of covariates on the design will be discussed.

Chapter 5. In this chapter a comparison is made between two different estimation methods for multilevel logistic models, which may be used for binary outcome variables. This comparison is made to choose the best estimation method for multilevel logistic models. Furthermore, the methods are also compared on basis of the distribution under the null hypothesis of the test statistic for testing the treatment effect. This distribution is often assumed to be the standard normal. Since in Chapter 6 statistical power is calculated as a function of this distribution, it has to be determined whether this assumption is valid.

Chapter 6. Outcome variables of intervention studies are often measured on a binary scale, and typically analyzed with logistic regression to allow the inclusion of covariates into the model. The same three design issues as for linear regression in Chapter 3 can also be addressed. for logistic regression. The logistic regression model is non-linear, and optimal designs may be obtained by linearization. An analytical solution to the optimal design can only be obtained with the Marginal Quasi Likelihood linearization method, which is known to produce biased parameter estimates. Therefore a simulation study was performed to translate the analytical results to results for Penalized Quasi Likelihood and estimation by numerical integration.

Chapter 7. In this chapter the results on optimal designs presented in Chapter 3, 4, and 6 are illustrated with an example. It will be shown that statistically optimal designs are not 
always feasible in practice because of, for example, a limited number of clusters and limited cluster sizes. This chapter shows that different outcome variables may lead to different optimal designs. It will also be shown that some information on the budget and study costs, the parameter values; and the drop-out rate is necessary to calculate the optimal design. The optimal design will be compared with designs that follow from practical limitations and a less expensive design. It will be shown that suboptimally planned experiments may lead to lower statistical power to detect a relevant treatment effect.

\section{References}

Aitkin, M., \& Longford, N. (1986). Statistical modelling issues in school effectiveness studies. Journal of the Royal Statistical Society, 149, 1-43.

Atkinson, A. C. \& Donev, A. N. (1996). Optimum experimental design. Oxford: Clarendon Press.

Bryk. A. S., \& Raudenbush, S. W. (1992). Hierarchical linear models. Newbury Park: Sage Publications.

Bryk, A. S., Raudenbush, S. W., \& Congdon, R. T., Jr. (1996). HLM: Hierarchical Linear and Nonlinear Modeling with the HLM/2L and HLM/3L Programs. Chicago: Scientific Software Intemational.

Dempster, A. P., Rubin, D. B., \& Tsutakawa, R. K., (1981). Estimation in covariance component models. Journal of the American Statistical Association, 76, 341-353.

Eisenhart, C. (1947). The assumptions underlying the analysis of variance. Biometrics, 3, 1-21.

Gibbons, R. D., \& Hedeker, D. (1997). Random effects probit and logistic regression models for threelevel data. Biometrics, 53, 1527-1537.

Goldstein, H. (1986). Multillevel mixed linear model analysis using iterative generalized least squares, Biomelrika, 73, 43-56.

Goldstein, H. (1989). Restricted unbiased iterative generalized least squares estimation, Biometrika, 76, $622-623$.

Goldstein, $\mathbb{H}$. (1991). Nonlinear multilevel models, with an application to discrete response data. Biometrika, 78, 45-51.

Goldstein, H. (1995). Multilevel statistical models, 2nd ed. London: Edward Amold.

Goldstein, $H_{i}$, \& Rasbash, $J_{i}$ (1996). Improved approximations for multilevel models with binary responses. Jowrnal of the Royal Statistical Soctety, Series A, 159, 505-513.

Goldstein, $\mathrm{H}_{2}$ Rasbash, J, Plewis, I, Draper, D., Browne, W., Yang, M., Woodhouse, G., \& Healy, M. (1998). A user's guide 1o MLwiN. London: Institute of Education.

Hartley, H. O., \& Rao, J. N. K. (1967). Maximum likelihood estimation for the mixed analysis of variance model. Biometrics, 23, $93-108$.

Hedeker, D., \& Gibbons, R. D. (1994). A random-effects ordinal regression model for multillevel analysis. Biometrics, 50, 933-944.

Hedeker, D., \& Gibbons, R. D. (1996a). MLXREG: a computer program for mixed-effects regression analysis with autocorrelated errors. Computer Methods and Programs in Biomedicine, 49, 229252.

Hedeker, D., \& Gibbons, R. D. (1996b). MIXOR: a computer program for mixed-effects ordinal regression analysis. Computer Methods and Programs in Blomedicine, 49, 157-176.

Hedeker, D., Gibbons, R. D., \& Davis, J. M. (1991). Random regression models for multicenter clinical trials data, Psychopharmacology Bulletin, 27, 73-77.

Hedeker, D., Gibbons, R. D., \& Flay, B. R. (1994), Random-effects regression models for clustered data with an example from smoking prevention research. Jowrnal of Consulting and Clinical Psychology, 62, 757-765.

Henderson, C. R. (1953). Estimation of variance and covariance components. Biometrics, 9, 226-252. 
Hox, J. J. (1994) Applied multilevel analysis. Amsterdan: TT-Publikaties.

Kreft, I., \& De Leeuw, J. (1998). Introducing multilewel modelling. Sage Publications, Londion.

Laird, N. M., \& Ware, J. H. (1982). Random-effects models for longitudinal data. Biometrics, 38, 963 974.

LaMotte, L. R. (1973). Quadratic estimation of variance components. Biometrics, 29, $311-330$.

Lindley, D. V., \& Smith, A. F. M. (1972). Bayes estimates for the linear model. Journal of the Royal Statistical Society, Series $B, 34,1-41$.

Longford, N. T. (1987). A fast scoring algorithm for maximum likelihood estimation in unbalanced models with nested random effects. Biometrika, 74, 817-827.

Longford, N. T. (1995). Random coefficien models. Oxford: Clarendon Press.

Mason, W. M., Wong, G. Y., \& Entwisle, B. (1984). The multilevel linear model: A better way to do contextual analysis. In: K. F. Schuessler (Ed.), Sociological Methodology, (pp. 72-103). London: Jossey Press.

Patterson, H. D., \& Thompson, R. (1971). Recovery of inter-block information when block sizes are unequal. Biometrika, 58; 545-554.

Rao, C. R. (1970). Estimation of heteroscedastic variances in linear modelis. Joumal of the Anericam Statistical Association, 65, 161-172.

Rao, C. R. (1971a). Estimation of variance and covariance components - MINQUE theory. Journal of Multivariate Analysis, 1, 257-275.

Rao, C. R. (1971b). Minimum variance quadratic unbiased estimation of variance components. Journa! of Multivariate Analysis, 1, 445-456.

Rao, C. R. (1972). Estimation of variance and covariance components in linear models. Journal of the American Statistical Association, 67, 112-115.

Silvey, S. D. (1980). Optimal design: an introdwction to the theory for parameter estimation. London: Chapman and Hall.

Snijders, T. (1996). Analysis of longitudinal data using the hierarchical linear model. Quality and Quantity, 30, 405-426.

Snijders, T. A. B., \& Bosker, R. J. (1999). Multilevel analysis: An introduction to basic and advanced multilevel modelling. London: Sage Publications. 


\title{
Chapter 2
}

\section{A review of four different methods for the analysis of multilevel experimental data}

\begin{abstract}
This paper reviews three traditional methods, namely the fixed effects model, the disaggregated data model, and the aggregated data model for the analysis of experimental data where individuals are nested within clusters, and compares these methods with the multilevel (mixed effects) model. The comparison is made for continuous outcomes, and is based on the estimator of the treatment effect and its variance, since these usually are of main interest in experiments. When the results of the experiment have to be valid for a larger population of clusters, the clusters in the experiment have to represent a random sample from this population and the multilevel model is preferably used for the analysis of the data. The three traditional methods have the same treatment effect estimator as the multilevel model if cluster sizes do not vary and there are no covariates. The variance of this estimator, however, is often underestimated by the fixed effects model, and underestimated or owerestimated by the disaggregated data model. For unbalanced designs and models with covariates the treatment effect as well as its variance may be estimated incorrectly by the traditional models. Under certain conditions the aggregated data model may be used as an alternative for the multilevel model.
\end{abstract}

Key words: multillevel model, fixed effects model, disaggregated data model, aggregated data model, mixed effects ANOVA, mixed effects regression

Acknowledgments: We wish to thank Brian R. Flay for his permission to use the TVSFP data, which were collected with funding from the National Institute of Drug Abuse, Grant 1-R01-DA03468 to Brian R. Flay, W.B. Hansen, and C.A. Johnson. 


\subsection{Introduction}

Experiments are developed to compare different treatments in terms of outcome variables measuring the health or behavior of individuals. In this paper we will focus on experiments where the data have a nested or hierarchical structure in which individuals are nested within clusters. For example, in the clinical trial analyzed by Hedeker, Gibbons, and Davis (1991) on the effect of different antipsychotics on the mental health, patients were nested within centers. In the trial by Bass, McWinney, and Donner (1986) where a new approach for the detection and managing of hypertension was studied, patients were nested within family practices. In the study by Sommer et al. (1986) on the effect of vitamin A supplementation on childhood mortality in northern Sumatra, children were nested within villages, and in the smoking cessation intervention by Hedeker, McMahon, Jason, and Salina (1994) employees were nested within worksites. Outcomes of individuals within the same cluster are likely to be correlated, i.e. there will be intra-ciluster correlation.

The effect of an active treatment can be estimated with a regression model, in which the outcome variable is regressed on treatment condition and relevant covariates, or with an analysis of variance (ANOVA) model. In the literature, several types of regression models are being used for multilevel experimental data. Three traditional regression models are the fixed effects model, the disaggregated data model, and the aggregated data model In the fixed effects model ${ }_{y}$ clusters are treated as fixed and their differences are taken into account by dummy coding in the regression model. In the disaggregated data model individuals are the unit of analysis and their nesting within clusters, i.e. the dependency among the outcomes of individuals within a cluster, is ignored. The aggregated data model is based upon aggregation of data within the same treatment condition to the cluster level, and clusters are thus the unit of analysis.

The multilevel model (Goldstein, 1995; Hox, 1994; Kreft and De Leeuw, 1998; Snijders and Bosker, 1999) treats individuals as the unit of analysis, but also takes into account the dependency of outcomes of individuals nested within the same cluster. This model is also referred to as mixed effects regression, random coefficient model (Longford, 1995) or hierarchical linear model (Bryk and Raudenbush, 1992), and assumes the clusters and individuals to represent random samples from the corresponding populations. Under this assumption cluster and person effects must be treated as random effects in the regression model, while treatment condition and covariates may be included as fixed effects.

Ideally, the aim of experiments in a hierarchically structured population should be to produce results which are not only valid for the clusters involved in the experiment, but also for a larger population of clusters. In that case the clusters involved in the experiment have to represent a random sample from the population of clusters, and multilevel analysis is the best method of analysis. In practice, there may be good reasons for treating clusters as fixed, for instance when the number of clusters in the experiment is very small, for details see Senn (1998). In this paper, however, we will focus on the situation where the clusters involved in 
the experiment may be considered a random sample from a much larger population of clusters.

The multilevel model is more complex than the more traditional models, and consequently investigators may still want to use these traditional models, even if they want to generalize the results from their experiment to all clusters in the population. Therefore a comparison between the traditional models and the multilevel model for experiments in hierarchically structured populations is relevant. In this paper, the relationship between the four models will be given and it will be shown under which circumstances the traditional methods are acceptable, and when and how they may lead to incorrect results. This will be done both in terms of regression and ANOVA models. ANOVA is familiar to many researchers and works well when cluster sizes do not vary, but encounters more difficulties with varying cluster sizes. The comparison made in this paper is based on analytical expressions for the estimator of the treatment effect and its variance, since these are of main interest in experiments, and is done for models with continuous outcomes, two levels of nesting (individuals within clusters), and with randomization at either level. Clusters will be randomly allocated to the treatment conditions for randomization at the cluster level, and all individuals within each cluster receive the same treatment condition. For randomization at the individual level, half of the individuals within a cluster will be randomized to the treatment group while the others will be randomized to the control group.

Part of the comparison has already been made by others, but has been published fragmentarily in various papers. In the present paper, these results will be presented systematically, and some gaps in knowledge will be filled up. From the literature it is known that:

1. Multilevel analysis is equivalent to a mixed model ANOVA when cluster sizes do not vary (Raudenbush, 1993).

2. The fixed effects model gives a smaller variance of the treatment effect estimator than the multilevel model (Sern, 1998; Gould, 1998; Jones, Teather, and Lewis, 1998) when randomization is done at the individual level and there is interaction between treatment and cluster.

3. For individual level randomization and no interaction between treatment and cluster, the disaggregated data model overestimates the variance of the treatment effect (Parzen, Lipsitz, and Dear, 1998). A similar phenomenon has been shown to occur in longitudinal studies with repeated measurements nested within persons instead of individuals within clusters (Dunlop, 1994).

4. The aggregated data model and the multilevel model lead to the same resuits if the design is balanced and randomization is done at the cluster level (Hopkins, 1982).

5. The disaggregated data model underestimates the variance of the treatment effect (Hedeker et al., 1994; Longford, 1995) when randomization is done at the cluster level, especially when the number of individuals within clusters and/or the intra- 
cluster correlation is large (Barcikowski, 1981). Underestimation by the disaggregated data model may also occur in longitudinal studies (Dunlop, 1994).

6. For observational studies with unbalanced designs and covariates, it has been shown that the disaggregated data model underestimates variances of regression coefficients (Bryk and Raudenbush, 1992), and that the disaggregated and aggregated data model should not be used for the analysis of multilevel data (Aitkin and Longford, 1986).

Again, we want to stress that in this paper multilevel models and more traditional models for experimental data with persons within clusters are presented. Multilevel models may also be used for observational or longitudinal studies. Tutorials on multilevel models for observational studies (Sullivan, Dukes, and Losina, 1999) and longitudinal studies (Burton, Gurrin, and Sly, 1998) have recently appeared.

The remainder of this paper is as follows: in Section 2.2 an example data set of an experiment in a hierarchically structured population and two different designs for such experiments are given. In Section 2.3, the multilevel regression model is related to the mixed effects ANOVA model. The fixed effects model, the disaggregated data model and the aggregated data model are presented in Section 2.4 and related to their corresponding ANOVA models. In Section 2.5, the four models are used to analyze generated data sets and it is shown that these models lead to different results. This difference in results will be explained using analytical expressions in Section 2.6. In Sections 2.3 to 2.6 we assume balanced designs and no covariates, but in Section 2.7 these assumptions will be relaxed. In Section 2.8 some conclusions will be presented. The notation of Goldstein (1995) will be used throughout this paper.

\subsection{Designs and example data set}

In principle, randomization and implementation of the two treatments may be done at either level of the hierarchy. So two different designs may be distinguished: Design 1, where randomization is done at the individual level, and Design 2, where randomization is done at the cluster level. The latter is often referred to as cluster randomization. For non-varying cluster sizes we have a sample of $n_{2}$ clusters and $n_{1}$ individuals per cluster. In Design $1, \frac{1}{2} n_{1}$ individuals per cluster are randomized to the control group and the others are randomized to the treatment group; assume $n_{1}$ to be even. In Design $2, \frac{1}{2} n_{2}$ clusters are allocated to each treatment; assume $n_{2}$ to be even, and all individuals within the same cluster receive the same treatment. A graphical representation of these two designs is given in Figure 2.1 for four clusters. Data on both treatment conditions are available in each cluster for Design 1 and so the interaction between cluster and treatment condition can be estimated. This is not possible in Design 2, where data on only one treatment condition are available per cluster, i.e. the data on the other treatment condition are missing by design. So individual level randomization is 


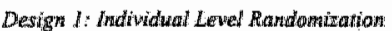

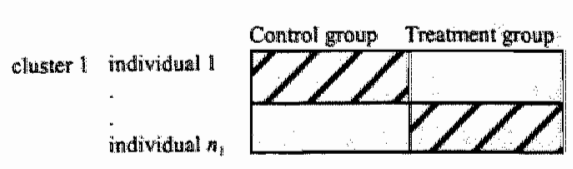

cluster 2 individual 1 individual $\boldsymbol{n}_{1}$

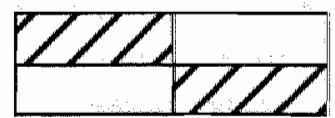

cluster 3 individual a

individual $m_{1}$

cluster 4 individual

individual $n_{8}$
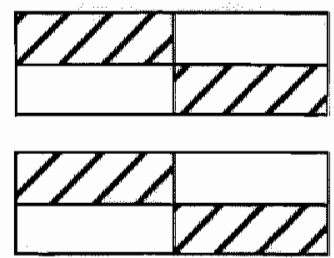

Design 2: Cluster Lewel Randontivention

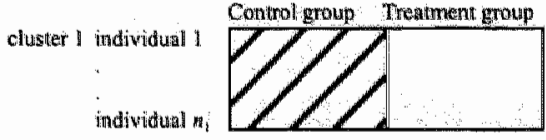

cluster 2 indiwidual I

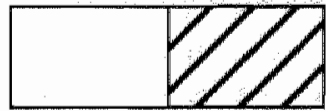

individual $n$

cluster 3 indixidual 1

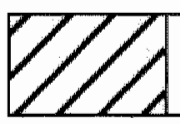

cluster 4 indiwidual 1

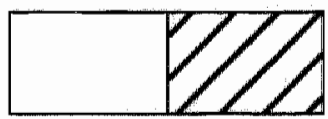

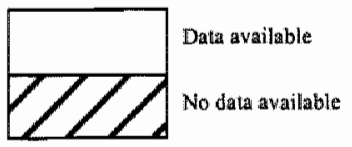

Figure 2.1. Graphical representation of Design 1 and Design 2.

to be preferred to cluster level randomization if treatment by cluster interaction is to be evaluated. Furthermore, randomization at the individual level results in more efficient estimates of the treatment effect (Moerbeek, Van Breukelen, and Berger, in press). Randomization at this level was done in, for example, the trial analyzed by Hedeker et al. (1991). In some experiments, however, randomization at the individual level is not possible and Design 2 will be the only alternative. For example, in the trial by Bass et al. (1986) randomization was done at the family practice level since it was recognized that the intervention would not function effectively if some patients in a practice were randomized to the treatment group and others not. In the study by Sommer et al. (1986), randomization was done at the village level since it was thought to be not acceptable to treat some children in a given village and others not. In the study by Hedeker et al. (1994) randomization at the employee levell would not have been possible because of treatment group contamination.

The results in this paper will be illustrated using a subset of data from the Television School and Family Smoking Prevention and Cessation Project (TVSFP) (Flay et al., 1988, 1995). This study was designed to test effects of a school-based social-resistance curriculum and a television-based program in terms of tobacco use prevention and cessation. Schools in Los Angeles and San Diego were randomized to one of five treatment conditions: (a) a socialresistance classroom curriculum, (b) a media (television) intervention, (c) a combination of 
these two, (d) a health-information-based attention-control curriculum, (e) a no-treatment control group. The dependent variable we used in the analyses is the post-intervention Tobacco and Health Knowledge Scale (THKS) score, which was the number of items that a student correctly answered in a seven item questionnaire to assess student tobacco and health knowledge. The data have a nested structure with pupils nested within classes within schools, and varying numbers of pupils per class and classes per school. In this paper we will restrict ourselves to two levels of nesting (pupils within classes) and two treatment conditions (media (television) intervention group and no-treatment control group), and only data from Los Angeles schools are considered. There were seventy classes and 837 pupils who met these conditions.

To illustrate the results in Sections 2.3 to 2.6 data sets with non-varying numbers of pupils per class were generated, using the parameter estimates from the analysis of the real data set as input values for the simulation. In Section 2.7, varying class-sizes and the use of covariates will be addressed and the real data will be analyzed.

\subsection{Multilevel regression model and mixed effects ANOVA model}

\subsubsection{Design 1: Randomization at the pupil level}

\subsubsection{Multilevel regression model}

In multilevel modelling, regression equations are formulated for each level of the multilevel data structure, and are then combined into a single equation model. For randomization at the pupil level, the THKS score denoted $y_{i j}$ and treatment condition denoted $x_{i j}$ of pupil $i$ in class $j$ are related by the pupil level model:

$$
y_{i j}=\beta_{0 j}+\beta_{1 j} x_{i j}+e_{i j}
$$

where $e_{i j}$ is a random error term at the pupil level. In this paper $x_{i j}=-1$ for the control group and $x_{i j}=+1$ for the media group. So, $\beta_{0 j}$ is the mean of $y_{i j}$ within class $j$ and $\beta_{1 j}$ is half the difference in outcorne between the two treatments within class $j$. The intercept and slope may vary across classes, randomly and/or as a function of class level covariates. This section will be restricted to models without any covariate, leaving the inclusion of covariates to Section 2.7. Thus, $\beta_{0 j}=\beta_{0}+u_{0 j}$, and $\beta_{1 j}=\beta_{1}+u_{1 j}$, where $\beta_{0}$ is the overall mean, $\beta_{1}$ is half the overall treatment effect, and $u_{0 j}$ and $u_{1 j}$ are random error terms representing the deviation of class $j$ from the overall mean and overall treatment effect, respectively. Substituting $\beta_{0 j}$ and $\beta_{1 j}$ into model (2.1) yields the single equation model:

$$
y_{i j}=\beta_{0}+\beta_{1} x_{i j}+u_{0 j}+u_{1 j} x_{i j}+e_{i j}
$$

The randorn effects $u_{0 j}, u_{1 j}$, and $e_{i j}$ are assumed to be independently and normally distributed with zero mean and variances $\sigma_{u 0}^{2}, \sigma_{u \mathrm{l}}^{2}$ and $\sigma_{e}^{2}$, respectively. To compare to ANOVA and for 
simplicity, a zero correlation between the random intercept and random slope is assumed in this paper (i.e. $\sigma_{u 01}=0$ ). But even when $\sigma_{u 01} \neq 0$ this independence restriction does not affect estimation and testing of the parameters if there are $\frac{1}{2} n_{1}$ pupils per treatment per class and if $x_{i j}$ in the multilevel model in (2.2) is centered around zero (Raudenbush, 1993). We will address this assumption further in Section 2.6. The inclusion of random effects at each level of the multilevel data structure leads to the decomposition of the variance of a pupils THKS score $y_{i j}$ into variance and covariance components, and correlated THKS scores of two pupils $i$ and $i$ ' within the same class:

$$
\begin{aligned}
& \operatorname{Var}\left(y_{i j}\right)=\sigma_{u 0}^{2}+\sigma_{u 1}^{2} x_{i j}^{2}+\sigma_{e}^{2} \text {, for each } i, j \text { if } \sigma_{u 01}=0 \\
& =\sigma_{u 0}^{2}+\sigma_{u 1}^{2}+\sigma_{e}^{2} \text { since } x_{i j}=-1 \text { or }+1 \\
& \operatorname{Cov}\left(y_{i j^{\prime}} y_{i j}\right)=\sigma_{w 0}^{2}+x_{i j^{\prime}} x_{i j} \sigma_{u 1}^{2} \text {, for each } i \neq i^{\prime} \text {, if } \sigma_{u 01}=0 \\
& =\sigma_{w 0}^{2}+\sigma_{u 1}^{2} \text { if } x_{i j}=x_{i^{\prime} j} \text { and } \sigma_{u 01}=0 \\
& =\sigma_{u 0}^{2}-\sigma_{u 1}^{2} \text { if } x_{y j^{\prime}} \neq x_{i^{\prime} j} \text { and } \sigma_{u 01}=0 \text {. }
\end{aligned}
$$

So, the Ordinary Least Squares (OLS) estimator of $\beta_{\|}$, which assumes independent THKS scores, should not be used, except if both $\sigma_{u 0}^{2}$ and $\sigma_{u 1}^{2}$ are equal to zero. The Generalized Least Squares (GLS) estimator of $\beta_{1}$ can be used if the variance and covariance components are known. In most cases, however, the variance components are unknown and Maximum Likelihood (ML, Hartley and Rao, 1967) or Restricted Maximum Likelihood (REML, Patterson and Thompson, 1971) estimation is required. See Searle, Casella and McCulloch (1992) for a description of these methods. It can be shown that in case of normally distributed outcomes ML and REML estimation correspond to Goldsteins Iterative Generalized Least Squares (IGLS, 1986) or Restricted Iterative Generalized Least Squares (RIGLS, 1989), respectively. Finally, the null hypothesis of no treatment effect may be tested using the test statistic $F=t^{2}=\beta_{1}^{2} / V \hat{a r r}\left(\beta_{1}\right)$, which, under the null hypothesis, has an $F$ distribution with 1 and $n_{2}-1$ degrees of freedom (Bryk and Raudenbush, 1992) when RIGLS is used. For models with a fixed slope $\beta_{1}$ (i.e. without interaction between class and treatment, $\sigma_{z 1}^{2}=0$ ), this test statistic has an $F$ distribution with 1 and $n_{1} n_{2}-n_{2}-1$ degrees of freedom under the null hypothesis when RIGLS is used. The degrees of freedom are equal to those for the ANOVA models in the next section since RIGLS estimates are equal to REML estimates under the normal case (see Goldstein, 1989), and for balanced data the solutions of the REML equations are equal to ANOVA estimators, whether normality is assumed or not (see for more details Searle, Casella, and McCulloch, 1992, Sections 4.8 and 6.6f). 


\subsubsection{Mixed effects ANOWA model}

The multillevel model in (2.2) can also be expressed as a mixed effects ANOVA model. We have a factorial design where classes and treatment conditions are crossed for Design 1. There are two treatments, $n_{2}$ classes, and $\frac{1}{2} n_{1}$ pupils per treatment in each class. The ANOVA model for pupil $i$ within class $j$ and treatment $t$ is given by

$$
y_{i j t}=\mu+\alpha_{t}+u_{j}+(\alpha u)_{j t}+e_{j j}
$$

where $\mu$ is the grand mean, $\alpha_{i}$ is the fixed effect associated with the $t$-th treatment, $u_{j}$ is the random effect associated with the $j$-th class, $(\alpha w)_{j t}$ is the random interaction effect, and $e_{j j t}$ is the random error term at the pupil level. If $t=2$ for the treatment group and $t=1$ for the control group, the correspondence between the parameters in the mixed effects ANOVA model (2.4) and the parameters in the multilevel regression model (2.2) is given by

$$
\mu=\beta_{0}, \quad \frac{\alpha_{2}-\alpha_{1}}{2}=\beta_{1}, \quad u_{j}=u_{0 j}, \quad \frac{(\alpha u)_{j}-(\alpha u)_{j 1}}{2}=u_{i j} \quad e_{i j t}=e_{i j}
$$

In principle there are two possibilities for the mixed effects ANOVA model: one without restrictions on the interaction terms, and one where $\Sigma_{t}(\alpha u)_{j t}=0$ for all $j$ (Searle et al., 1992, section 4.3). This paper assumes the latter because this also applies to the multilevel model (since $\Sigma_{i} x_{i j} u_{\mathrm{i} j}=0$ for all $j$ since there are only two treatment conditions which are coded -1 and +1 , and there are $\frac{1}{2} n_{1}$ pupils per treatment per class). As in the multilevel model in (2.2), the random terms $u_{j},(\alpha u)_{j l}$, and $e_{j y}$ are independently and normally distributed with zero mean and variances $\sigma_{w 0}^{2}, \sigma_{u 1}^{2}$, and $\sigma_{z}^{2}$, respectively. Note that the mixed effects ANOVA model assumes that the class effect and the treatment-by-class interaction are independent, which is not necessarily the case for multilevel models, where $\sigma_{w 01}$ may be unequal to zero. The variance components in ANOVA models are estimated by equating the Mean Squares (MS) to their expected values. In the lower half of Table 2.1, the expected Mean Squares $\mathrm{E}\left(M S_{\text {mixed }}\right)$ for the mixed effects factorial ANOVA model are given. The null hypothesis of no treatment effect can be tested using the test statistic $F=M S_{\text {treatment }} / M S_{\text {interaction }}$ which has an $F$ distribution with 1 and $n_{2} \mathbb{1}$ degrees of freedom under the null hypothesis. With no interaction between class and treatment (i.e. $\sigma_{u 1}^{2}=0$ ), the Sum of Squares $S S_{\text {interaction }}$ is pooled with $S S_{\text {errorn, }}$ and the test statistic becomes $F=M S_{\text {treatment }} / M S_{\text {error }}$ which has an $F$ distribution with 1 and $n_{1} n_{2}-n_{2}-1$ degrees of freedom under the null hypothesis. As will be shown in the following example, the mixed effects ANOVA model gives the same results as the multilevel regression model. 


\section{Example: Analyses of data set for Design 1}

In this paper we will restrict ourselves to those Los Angeles schools which were randomized to either the media or no-treatment intervention group. Analysis of the data with two levels of nesting (pupils within classes) gave $\beta_{0}=2.34, \hat{\beta}_{1}=0.12, \sigma_{t 0}^{2}+\sigma_{u l}^{2}=0.16$, $\hat{\sigma}_{e}^{2}=1.72$. Note that the variance components cannot be estimated separately as there was only one treatment condition per class, see Section 2.3.2. For illustrative purposes we generated data for $n_{2}=70$ classes with $n_{1}=12$ pupils each, using the estimated regression coefficients and variance components from the real data set (with $\sigma_{w 0}^{2}=0.1$ and $\sigma_{w 1}^{2}=0.06$ ). These data were analyzed using the multilevel model and with REML estimation and using ANOVA estimation. The results of the analyses are presented in Table 2.1, showing that both approaches give the same estimated regression coefficients and variance components, and both do not reject the null hypothesis of no treatment effect at the five percent level.

Table 2.1 Results for Data Set for Design 1

Results based on the multilevel model

Parameter $\quad$ Estimate Standard Error $\quad F=t^{2} \quad p$

Fixed effects:

Intercept, $\beta_{0}$

$2.304 \quad 0.058$

Treatment effect, $\beta_{1} \quad 0.097 \quad 0.050$

3.776

0.056

Random effects:

Random intercept, $\sigma^{2} \quad 0.096$

Random slope, $\sigma_{u 1}^{2} \quad 0.039$

Random error term, $\sigma_{g}^{2} \quad 1.634$

Results based on the mixed effects ANOVA model

\begin{tabular}{|c|c|c|c|c|c|c|c|}
\hline Source & $d f$ & & $S S$ & $M S=S S / d f$ & $\mathrm{E}\left(M S_{\text {mixed }}\right)$ & $F$ & $p$ \\
\hline Treatment & 1 & $=1$ & 7.947 & 7.947 & $\sigma_{e}^{2}+n_{1} \sigma_{u 1}^{2}+\frac{1}{2} n_{1} n_{2} \sigma_{\alpha}^{2}$ & 3.776 & 0.056 \\
\hline Class & $n_{2}-1$ & $=69$ & 192.148 & 2.785 & $\sigma_{0}^{2}+n_{1} \sigma_{u 0}^{2}$ & & \\
\hline Interaction & $n_{2}-1$ & $=69$ & 145.224 & 2.105 & $\sigma^{2}+n_{1} \sigma_{u 1}^{2}$ & & \\
\hline Error & $n_{1} n_{2}-2 n_{2}$ & $=700$ & 1144.088 & 1.634 & $\sigma^{2}$ & & \\
\hline Total & $n_{1} n_{2}-1$ & $=839$ & 1489.407 & & & & \\
\hline
\end{tabular}

$\hat{\sigma}_{\text {wi } 0}^{2}=\left(M S_{\text {cliass }}-M S_{\text {error }}\right) / 12=0.096, \quad \hat{\sigma}_{u \| l}^{2}=\left(M S_{\text {interaction }}-M S_{\text {ernor }} y / 12=0.039, \quad \hat{\sigma}^{2}=M S_{\text {error }}=1.634\right.$ $\hat{\mu}=\bar{y}_{y}=2.304, \quad\left(\hat{\alpha}_{2}-\hat{\alpha}_{1}\right) / 2=\left(\bar{y}_{.2}-\bar{y}_{.1}\right) / 2=0.097$

Note. It is assumed that $\Sigma_{i}(\alpha u)_{j t}=0$. Without this restriction $n_{1}$ has to be replaced by $\frac{1}{2} n_{1}$ in the $\mathrm{E}(M S)$ for treatment and interaction (Searle et al., 1992, p. 123-126). 


\subsubsection{Design 2: Randomization at the class level}

\subsubsection{Multilevel regression model}

The multilevel regression model for Design 2 is given by

$$
y_{b j}=\beta_{0}+\beta_{1 j} x_{i j}+u_{j}+e_{i j}
$$

where the random interaction term $x_{i j} u_{i j}$ in $(2.2)$ is omitted since all pupils within the same class receive the same treatment. As a result $\sigma_{w 0}^{2}$ and $\sigma_{t u}^{2}$ cannot be estimated separately. Instead, their sum is estimated which will be coded as $\sigma_{u}^{2}=\sigma_{u 0^{*}}^{2}+\sigma_{u 1}^{2}$ in this paper. Furthermore $x_{i j}$ may be replaced by $x_{j}$ since treatment condition does not vary within classes, and again treatment condition is coded as $x_{j}=+1$ for the treatment group and $x_{j}=-1$ for the control group. The null hypothesis of no treatment effect is tested by the test statistic $F=t^{2}=$ $\beta_{1}^{2} / \operatorname{Var}\left(\beta_{1}\right)$ which, under the null hypothesis, has an $F$ distribution with 1 and $n_{2}-2$ degrees of freedom (Bryk and Raudenbush, 1992) when RIGLS is used. The degrees of freedom are equal to those for the ANOVA model in the next section (see p. 19 for the explanation).

\subsubsection{Mixed effects ANOVA model}

For randomization at the class level, we have a mixed effects nested ANOVA model in which classes are nested within treatments:

$$
y_{i j t}^{\prime}=\mu+\alpha_{t}+u_{j t}+e_{i j t^{\prime}}
$$

where $\mu$ is the grand mean, $\alpha_{f}$ is the fixed effect associated with the $t$-th treatment, and $u_{j t}$ and $e_{i j}$ are the random terms at the class and pupil level which are assumed to be independently and normally distributed with zero mean and variances $\sigma_{u}^{2}$ and $\sigma_{e}^{2}$, respectively. There are $\frac{1}{2} n_{2}$ classes per treatment and $n_{1}$ pupils per class. If $t=2$ for the treatment group and $t=1$ for the control group, the correspondence between the mixed effects ANOVA model in (2.7) and the multilevel regression model in (2.6) is given by

$$
\mu=\beta_{0}, \quad \frac{\alpha_{2}-\alpha_{1}}{2}=\beta_{1}, \quad u_{j t}=u_{j}, \quad e_{i j t}=e_{i j t}
$$

The expected Mean Squares for the ANOVA model in (2.7), $\mathrm{E}\left(M S_{\text {mixed }}\right)$, are given in the lower half of Table 2.2. The test statistic for the null hypothesis of no treatment effect is given by $F=M S_{\text {treatment }} / M S_{\text {elass }}$ which, under the null hypothesis, has an $F$ distribution with 1 and $n_{2}-2$ degrees of freedom. 
Table 2.2 Results for Data Set for Design 2

\begin{tabular}{lllll}
\hline \hline \multicolumn{4}{l}{ Results based on the multilevel model } & \\
\hline Parameter & Estimate & Standard Error & $F=t^{2}$ & $p$ \\
\hline Fixed effects: & & & & \\
Intercept, $\beta_{0}$ & 2.256 & 0.073 & & \\
Treatment effect, $\beta_{1}$ & 0.166 & 0.073 & 5.221 & 0.025 \\
Random effects: & & & & \\
Random intercept, $\sigma_{u t}^{2}$ & 0.212 & & & \\
Random error term, $\sigma_{e}^{2}$ & 1.905 & & & \\
\hline
\end{tabular}

Results based on the mixed effects ANOVA model

\begin{tabular}{llllllll}
\hline Source & $d f$ & & $S S$ & $M S=S S / d f$ & $\mathrm{E}\left(M S_{\text {mixed }}\right)$ & $F$ & $p$ \\
\hline Treatment & 1 & $=1$ & 23.231 & 23.231 & $\sigma_{e}^{2}+n_{1} \sigma_{u}^{2}+\frac{1}{2} n_{1} n_{2} \sigma_{\alpha}^{2}$ & 5.221 & 0.025 \\
Classes within & $n_{2}-2$ & $=68$ & 302.599 & 4.450 & $\sigma_{e}^{2}+n_{1} \sigma_{u}^{2}$ & & \\
$\quad$ treatments & & & & & & & \\
Error & $n_{1} n_{2}-n_{2}=770$ & 1466.541 & 1.905 & $\sigma_{e}^{2}$ & & & \\
Total & $n_{1} n_{2}-1$ & $=839$ & 1792.371 & & & & \\
\hline
\end{tabular}

$\hat{\sigma}_{u}^{2}=\left(M S_{\text {class }}-M S_{\text {eтто }}\right) / 12=0.212, \quad \hat{\sigma}_{e}^{2}=M S_{\text {etror }}=1.905$.

$\hat{\mu}=\bar{y}_{\ldots}=2.256, \quad\left(\hat{\alpha}_{2}-\hat{\alpha}_{1}\right) / 2=\left(\bar{y}_{.2}-\bar{y}_{.1}\right) / 2=0.166$

Example: Analyses of data set for Design 2

A data set was also generated for randomization at the class level with $n_{1}=12$ and $n_{2}=70$, using the estimated parameter values from the analysis of the real data as input for the generation process. The data were analyzed using the multilevel model with REML estimation and using ANOVA estimation. Again both methods produce the same estimated parameter values and test statistic and both reject the null hypothesis of no treatment effect at the five percent level, see Table 2.2 for the numerical results. Note that Design 1 shows a non-significant effect, while Design 2 shows a significant effect, which is a consequence of the fact that the generated effect for Design 2 is larger than the generated effect for Design 1 . 


\subsection{Traditional models}

Three more traditional regression models for the analysis of multilevel experimenta] data are the fixed effects model, the disaggregated data model and the aggregated data model. These models are presented in this section, together with their equivalent ANOVA models.

\subsubsection{Fixed effects regression model and fixed effects ANOVA model}

In contrast to the multilevel regression model, the fixed effects regression model includes class and interaction effects as fixed effects. For randomization at the pupil level (Design 1) we have

$$
y_{i j}=\beta_{0}+\beta_{1} x_{i j}+\sum_{k=1}^{m_{2}-1} \beta_{h+1} d_{h j}+\sum_{h=1}^{n_{2}-1} \beta_{n_{2}+k^{\prime}} x_{j} d_{h j}+e_{i j^{*}}
$$

where $y_{i j}$ is the THKS score, $x_{i j}$ denotes treatment condition, and $e_{i j}$ is the random error term at the pupil level with zero mean and variance $\sigma_{e}^{2}$. The classes may be represented by $n_{2}-1$ dummy variables $d_{h}$ and the $n_{2}-t h$ class is the reference class. The dummy variables are coded such that $d_{h j}=+1$ if $h=j_{x} d_{h j}=-1$ if $h=n_{2}$, and $d_{h j}=0$ otherwise, so that they are centered and $x_{i j} d_{h j}$ is orthogonal to $x_{i j}$ and $d_{l j}$. The fixed effects ANOVA model is given by $(2.4)$, where $u_{j}$ is now regarded a fixed effect. The corresponding expected mean squares are equal to those for the mixed effects ANOVA model in Table 2.1 except that $E\left(M S_{\text {treatment }}\right)=\sigma_{e}^{2}+\frac{1}{2} n_{1} n_{2} \sigma_{a t}^{2}$. The test statistic for the null hypothesis of no treatment effect is calculated as $F=\hat{\beta}_{1}^{2} / V a ̂ r\left(\hat{\beta}_{1}\right)=M S_{\text {trestment }} / M S_{\text {error }}$ and has 1 and $n_{1} n_{2}-2 n_{2}$ degrees of freedom under the null hypothesis. The terms $x_{i j} d_{h i}$ may be deleted from model (2.9) if there is no interaction between treatment and class, and the $S S_{\text {interaction }}$ is pooled with the $S S_{\text {enror }}$ Then, the null hypothesis is

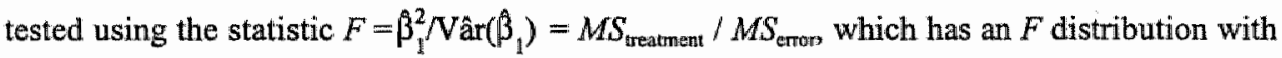
1 and $n_{1} n_{2}-n_{2}-1$ degrees of freedom under the null hypothesis.

There are one reference class and $\frac{1}{2} n_{2}-1$ centered dummy variables for each treatment condition and no interaction terms in the fixed effect regression model in (2.9) for randomization at the class level. The fixed effects ANOVA model is given by $(2.7)$, with $u_{j t}$ being a fixed effect. The corresponding expected mean squares are equal to those for the mixed effects ANOVA model in Table 2.2 except that $E\left(M S_{\text {treament }}\right)=\sigma_{*}^{2}+\frac{1}{2} n_{1} n_{2} \sigma_{\alpha}^{2}$. The test statistic for the null hypothesis is calcullated as $F=\hat{\beta}_{1}^{2} /$ Var $\left(\hat{\beta}_{1}\right)=M S_{\text {treatment }} / M S_{\text {error }}$ which has 1 and $n_{1} n_{2}-n_{2}$ degrees of freedom under the null hypothesis. 


\subsubsection{Disaggregated data model and one-way ANOVA model}

The disaggregated data model differs from the multilevel model in $(2.2)$ in that it contains only one random error term:

$$
y_{i j}=\beta_{0}+\beta_{1} x_{i j}+r_{i j}
$$

where $y_{i j}$ and $x_{i j}$ are the THKS score and treatment condition, respectively, and $r_{i j}$ is a random error term with zero mean and variance $\sigma_{r}^{2}$. Note that $r_{i j}=u_{0 j}+u_{i j} x_{i j}+e_{i j}$ if the mixed effect model is correct but the disaggregated data model is assumed. In that case the variance of a pupil's THKS score is equal to $\operatorname{Var}\left(y_{i j}\right)=\sigma_{r}^{2}=\sigma_{u 0}^{2}+\sigma_{u 1}^{2}+\sigma_{e}^{2}$, according to (2.3). However, the disaggregated data model. assumes that the $r_{i j}$ 's are independent, that is: $\operatorname{Cov}\left(y_{i j}, y_{i j}\right)=0$, for $i \neq i$, and so the disaggregated data model ignores dependence of THKS scores of pupils within the same class and the OLS estimator can be used in this case. Like in the muitilevel modelling approach, the test statistic for the test of no treatment effect is given by $F=t^{2}=$ $\hat{\beta}_{1}^{2} / \operatorname{Var}\left(\hat{\beta}_{1}\right)$, which is has an $F$ distribution with 1 and $n_{1} n_{2}-2$ degrees of freedom under the null hypothesis of no treatment effect.

The ANOVA model corresponding to the disaggregated data model in (2.10) is given by

$$
y_{i t}=\mu+\alpha_{t}+r_{i t}
$$

where $y_{i t}$ is the THKS score for the $i$-th pupil within the $t$-th treatment, $\mu$ is the grand mean, $\alpha_{t}$ is the fixed effect for the $t$-th treatment. The random error term at the pupil level $r_{i t}$ has variance $\sigma_{r}^{2}$. There are $\frac{1}{2} n_{1} n_{2}$ pupils per treatment. The null hypothesis of no treatment effect is now tested using the test statistic $F=M S_{\text {treatrent }} / M S_{\text {error }}$, which has an $F$ distribution with 1 and $n_{1} n_{2}-2$ degrees of freedom under the null hypothesis. Note that the disaggregated data model in (2.10) and the one-way ANOVA model in (2.11) both apply to Design 1 and Design 2 since they ignore class effects altogether.

\subsubsection{Aggregated data model}

The aggregated data model describes the data after aggregation (averaging) within the same treatment to the class level. This means that each cell in Figure 2.1 collapses to one average observation. Thus, if randomization is done at the pupill level, we have two correlated mean THKS scores per class, one for each treatment condition. Based upon (2.2) the regression models for the mean outcomes in the control and treatment group, denoted by $\bar{y}_{\text {.jc }}$ and $\bar{y}_{, j i}$, are given by

$$
\begin{aligned}
& \bar{y}_{j c}=\beta_{0}-\beta_{1}+u_{0 j}-u_{1 j}+\bar{e}_{j c} \\
& \bar{y}_{j t}=\beta_{0}+\beta_{1}+u_{0 j}+u_{1 j}+\bar{e}_{j k}
\end{aligned}
$$

respectively. The treatment effect per class can be estimated by 


$$
\hat{\beta}_{1 j}=\frac{\bar{y}_{j r}-\bar{y}_{j c}}{2},
$$

which has variance $\sigma_{u 1}^{2}+\sigma_{e}^{2} / n_{1}$. For equal class sizes the overall treatment effect is then estimated as the mean of the $\hat{\beta}_{1 y}$, which has variance $\left(n_{1} \sigma_{u 1}^{2}+\sigma_{e}^{2}\right) / n_{1} n_{2}$. To test the null hypothesis of no treatment effect the paired samples $t$-test with $n_{2}-1$ degrees of freedom under the null hypothesis can be used.

If randomization is done at the class level, there is one mean THKS score per class, $\bar{y}_{j,}$, which is related to treatment condition $x_{j}$, by

$$
\bar{y}_{j, j}=\beta_{0}+\beta_{1} x_{j}+u_{j}+\bar{e}_{j}
$$

where $\vec{e}_{j}$ is the class mean of the random effect at the pupil level. The mean THKS scores per class are assumed to be independently distributed with variance

$$
\operatorname{Var}\left(\bar{y}_{j}\right)=\operatorname{Var}\left(u_{j}+\bar{e}_{j}\right)=\sigma_{u}^{2}+\frac{\sigma_{e}^{2}}{n_{1}},
$$

and the OLS estimator can be used for the aggregated data model. The null hypothesis of no treatment effect can be tested with the independent samples $t$-test with $n_{2}-2$ degrees of freedom under the null hypothesis.

\subsection{Analyses of artificial data sets}

The two data sets from Section 2.3 were analyzed with the multilevel model, the fixed effects model, the disaggregated data model, and the aggregated data model. REML estimation was used for the multilevel model, and OLS estimation for all other models.

The results for Design 1 are given in the upper part of Table 2.3. This table shows that all models produce the same estimated treatment effect $\beta_{1}$, but that its standard error is underestimated by the disaggregated data model and the fixed effects model. As a result, the test statistics for these two models are somewhat larger than those for the multilevel model and the aggregated data model, and $p$-values for the fixed effects model and the disaggregated data model are too small. Note that for the aggregated data model $\sigma_{\varepsilon}^{2}$ cannot be disentangled from $\sigma_{u 1}^{2}$ since there are only two mean THKS scores within each class.

The results of the analysis for Design 2 are given in the lower part of Table 2.3, showing that the multilevel model and aggregated data model produce the same estimated treatment effect and standard error, whereas the latter is too small for the fixed effects model and the disaggregated data model. All models reject the null hypothesis of no treatment effect at the five percent level. Note that $\sigma_{e}^{2}$ cannot be disentangled from $\sigma_{u}^{2}$ for the aggregated data model since there is only one mean THKS score per class. 
Table 2.3 Results of Multilevel and Traditional Analyses of Data Sets

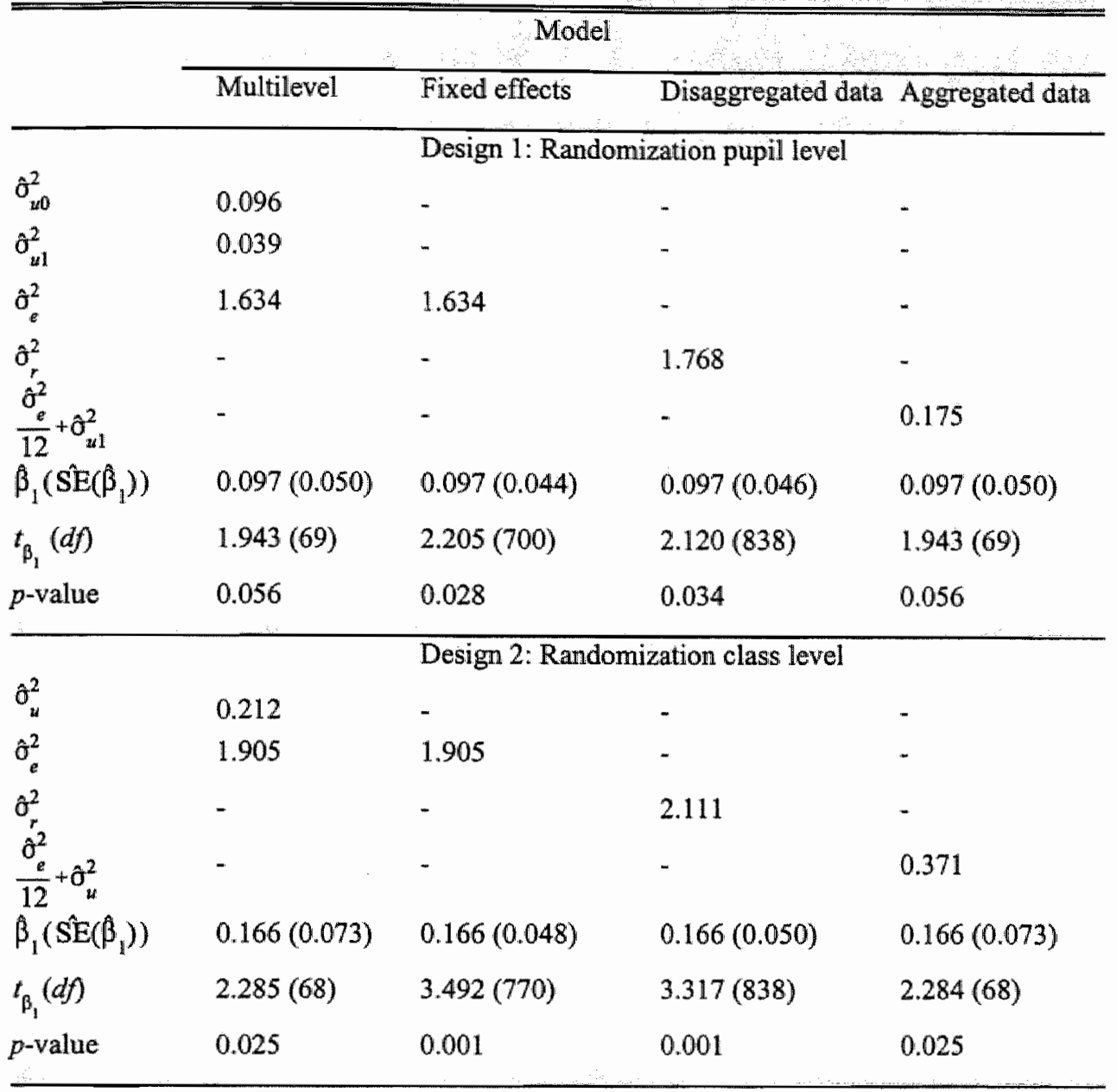

\subsection{Comparison of the four methods based on analytical expressions}

In this section the results in the previous section will be explained by means of analytical expressions. The four estimation methods will be compared with each other assuming non-varying class sizes and no covariates. When control and treatment groups are coded by $x_{i j}=-1$ and $x_{i j}=+1$, the estimator of the treatment effect $\beta_{1}$ is given by

$$
\hat{\beta}_{1}=\frac{\sum_{i j} x_{i j} y_{i j}}{n_{1} n_{2}}=\frac{\bar{y}_{. i}-\bar{y}_{. c}}{2},
$$

for each of the four models and for both levels of randomization. The means $\bar{y}_{. c}$ and $\bar{y}_{\ldots}$ are the mean THKS scores in the control and treatment groups, respectively. 
Table 2.4. Vâr $(\hat{\beta})$ for the four Regression Modells

\section{Model}

Level of randomization Multilevel Fixed effects Disaggregated data Aggregated data

Pupil (interaction treatment by class)

Pupil (no interaction treatment by class)

Class

Multilevel Fixed effects Disaggregated data

$\frac{\partial_{r}^{2}}{n_{1} n_{2}}=\frac{\sigma_{u 0}^{2}+\sigma_{u 1}^{2}+\sigma_{e}^{2}}{n_{1} n_{2}}$

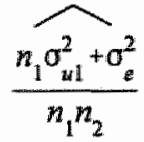

$$
\frac{\hat{\sigma}_{r}^{2}}{n_{1} n_{2}}=\frac{\widehat{\sigma_{u 0}^{2}+\sigma_{e}^{2}}}{n_{1} n_{2}}
$$$$
\frac{\widehat{n_{1} \sigma_{w 1}^{2}+\sigma_{e}^{2}}}{n_{1} n_{2}}
$$

$$
\frac{n_{1}^{\sigma_{0}^{2}+\sigma_{0}^{2}}}{n_{1} n_{2}}
$$

Note. Control and treatment group are denoted by $x_{i j}=-1$ and $x_{i j}=+1$, respectively.

For class level randomization $\sigma_{u}^{2}=\sigma_{u p}^{2}+\sigma_{u 1}^{2}$.

For the aggregated data model the variance components cannot be estimated separately.

Furthermore, for randomization at the pupil level the aggregated data model always assumes treatment by class interaction.

In Table 2.4 the $\operatorname{Var}\left(\hat{\beta}_{1}\right)$ for the four models are given. For randomization at the class level (Design 2) the aggregated data model gives the same $\operatorname{Var}\left(\hat{\beta}_{1}\right)$ and degrees of freedom as the multilevel model although the variance components cannot be estimated separately by the aggregated data model, while $\operatorname{Var}\left(\hat{\beta}_{1}\right)$ is underestimated by the fixed effects model and the disaggregated data model. The amount of underestimation by the disaggregated data model depends on $n_{1}$ and the intra-class correlation coefficient $\rho_{c}$, which measures the amount of variance between classes, i.e. $\rho_{c}=\sigma_{u}^{2} /\left(\sigma_{u}^{2}+\sigma_{v}^{2}\right)$. The ratio of $\operatorname{Var}\left(\beta_{1}\right)$ obtained with the multilevel model and $\operatorname{Var}\left(\beta_{1}\right)$ obtained with the disaggregated data model is given by

$$
\text { variance ratio }=\frac{\operatorname{Vâr}\left(\hat{\beta}_{1}\right)_{\text {multitevel }}}{\operatorname{Vâr}\left(\hat{\beta}_{1}\right)_{\text {disaggregated data }}}=\frac{n_{1} \hat{\sigma}_{u}^{2}+\hat{\sigma}_{e}^{2}}{\partial_{r}^{2}}=n_{1} \hat{\rho}_{c}+\left(1-\hat{\rho}_{c}\right)=\left(n_{1}-1\right) \hat{\rho}_{c}+1 \ldots
$$

From (2.17) it is clear that when $n_{1}$ and/or $\rho_{\mathrm{c}}$ increase, this ratio will also increase. Even for small intra-class correlation coefficients the underestimation of the variance of the treatment effect estimator with the disaggregated data model is considerable. For example, for $\rho_{c}=0.1$ and $n_{1}=30$, the $\operatorname{Var}\left(\beta_{1}\right)$ from the disaggregated data model is about four times as small as the $\operatorname{Var}\left(\beta_{1}\right)$ from the multilevel model. So, the confidence interval for $\beta_{1}$ in the disaggregated data model will be about twice as small as that obtained under the multilevel model, and the null hypothesis of no treatment effect will be rejected too often, leading to an inflation of the 
type I error rate. The amount of underestimation for the fixed effects model depends on the number of pupils per class and the intra-class correlation coefficient as well, as is shown by the variance ratio:

$$
\text { variance ratio } \equiv \frac{\operatorname{Vâr}\left(\hat{\beta}_{1}\right)_{\text {multilevel }}}{\operatorname{Vâr}\left(\hat{\beta}_{1}\right)_{\text {fixed effects }}}=\frac{n_{1} \hat{\sigma}_{z}^{2}+\hat{\sigma}_{c}^{2}}{\hat{\sigma}_{e}^{2}}=\frac{n_{1} \hat{\rho}_{c}+\left(1-\hat{\rho}_{c}\right)}{1-\hat{\rho}_{c}}=n_{1}\left(\frac{\hat{\rho}_{c}}{1-\hat{\rho}_{t a}}\right)+1 \text {, }
$$

and again the variance ratio increases with $n_{1}$ and $\rho_{c}$.

For randomization at the pupil level (Design 1) and assuming treatment by class interaction (i.e. a random slope), the aggregated data model produces the same $V a ̂ r\left(\hat{\beta}_{1}\right)$ and degrees of freedom as the multilevel model, although the variance components cannot be estimated separately by the aggregated data model. The $\operatorname{Var}\left(\hat{\beta}_{1}\right)$ is underestimated by the fixed effects model, and underestimated or overestimated by the disaggregated data model, depending on the number of pupils per class and the values of the variance components. So far, we assumed $\sigma_{w 01}=0$, but it can be shown that when the random intercept and slope are correlated the formulas in Table 2.5 still hold if there are $\frac{1}{2} n_{1}$ pupils per treatment per class and the $x_{i j}$ in (2.3) are centered around zero.

If randomization is done at the pupil level and there is no class by treatment interaction (i.e. no random slope: $\sigma_{u 1}^{2}=0$ ) then the $\operatorname{Vat}\left(\beta_{1}\right)$ for the disaggregated data model is greater than the $\operatorname{Var}\left(\hat{\beta}_{1}\right)$ for the multilevel model. As a result, confidence intervals for $\beta_{1}$ obtained with the disaggregated data model will be too wide and the null hypothesis that $\beta_{1}=0$ will be rejected less often, inflating the type II enror rate. The variance ratio is given by

$$
\text { variance ratio } \equiv \frac{\operatorname{Vâr}\left(\hat{\beta}_{1}\right)_{\text {multilevel }}}{\operatorname{Vâr}\left(\hat{\beta}_{1}\right)_{\text {disaggregated data }}}=\frac{\hat{\sigma}_{e}^{2}}{\hat{\sigma}_{r}^{2}}=1-\hat{\rho}_{c}
$$

which depends on $\rho_{c}$ but not on $n_{1}$. So, the overestimation of $\operatorname{Var}\left(\hat{\beta}_{1}\right)$ by the disaggregated data model may be ignored for small intra-class correlation coefficients (say $\rho_{c}<0.05$ ). Furthermore the fixed effects model produces the same results as the multilevel model, whereas the results for the aggregated data model (i.e. the paired samples $t$-lest) are inefficient. In fact the aggregated data model assumes interaction between treatment and class

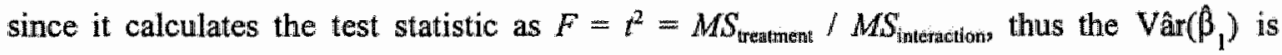
inefficiently but unbiasedly estimated and the degrees of freedom for the denominator for the test statistic are too low.

The conclusions in this section are presented schematically in Table 2.5, which also gives references where some of the conclusions have also been presented. 
Tabie 2.5. Comparison of Traditional Models to Multilevel Model with Respect to Estimated $\operatorname{Var}\left(\beta_{1}\right)$ and Degrees of Freedom of the Denominator for the F Distribution of the Test Statistic under the Null Hypothesis, Assuming Equal Class Sizes and Classes Represent a Random Sample

Analysis model

\begin{tabular}{|c|c|c|c|}
\hline Lewel of randomization & Fixed effects & Disaggregated data & Aggregated data \\
\hline $\begin{array}{l}\text { Pupil (initeraction } \\
\text { treatment by class) }\end{array}$ & $\begin{array}{l}\text { Underestimated } \operatorname{Var}\left(\beta_{1}\right), \\
\text { Senn (1998), Gould (1998), } \\
\text { Jones et al. (1998) } \\
\text { incorrect df: } n_{1} n_{2}-2 n_{2}\end{array}$ & $\begin{array}{l}\text { Underestimated or } \\
\text { overestimated } \operatorname{Var}\left(\beta_{1}\right) \text {, } \\
\text { depending on values } \\
\text { variance components. } \\
\text { incontect df: } n_{1} n_{2}-2\end{array}$ & $\begin{array}{l}\text { Correctly estimated } \operatorname{Var}\left(\beta_{1}\right) \\
\text { Equal to paired samples } t \text {-test } \\
\text { on class by treatment means. } \\
\text { correct df: } n_{2}-1\end{array}$ \\
\hline $\begin{array}{l}\text { Pupil (no interaction } \\
\text { treatment by chass) }\end{array}$ & $\begin{array}{l}\text { Correctly estimated } \\
\operatorname{Var}\left(\phi_{1}\right)\end{array}$ & $\begin{array}{l}\text { Overestimated } \operatorname{Var}\left(\beta_{1}\right) \text {, } \\
\text { Parzen el al }(1998)\end{array}$ & $\begin{array}{l}\text { Inefficiently estimated } \\
\operatorname{Var}\left(\beta_{1}\right) \text { because equal to } \\
\text { paired samples } t \text {-test on class } \\
\text { by treatment means which } \\
\text { assumes interaction. } \\
\text { unnecessarily low df: } n_{2}-1\end{array}$ \\
\hline \multirow[t]{2}{*}{ Class } & Underestimated $\operatorname{Var}\left(\beta_{1}\right)$ & $\begin{array}{l}\text { Underestimated } \operatorname{Var}\left(\beta_{1}\right) \text {, } \\
\text { Hedeker et al. }(1994) \text {, } \\
\text { Longford }(1995) \text {, } \\
\text { Barcikowski }(1981)\end{array}$ & $\begin{array}{l}\text { Correctly estimated } \operatorname{Var}\left(\beta_{1}\right) \\
\text { Hopkins (1982). Equal to } \\
\text { independent samples } t \text {-test on } \\
\text { class means. }\end{array}$ \\
\hline & uncorrect df: $n_{1} n_{2}-n_{2}$ & incorrect df: $n_{1} n_{2}-2$ & correct df: $n_{z}-2$ \\
\hline
\end{tabular}

\subsection{Generalization to more complex models}

The results in the previous section are limited to equal class sizes and models with no covariates. Equal class sizes may not always be feasible in practice, and in some cases covariates have to be included into the model. In this section, these restrictions will be relaxed one at a time.

\subsubsection{Varying class sizes}

In this section we will assume varying class sizes $n_{1, j}$, but $50: 50$ randomization to the treatment and control group. So, there are $\frac{1}{2} n_{1 j}$ pupils per treatment in class $j$ for randomization at the pupil level, and $\frac{1}{2} n_{2}$ classes per treatment for randomization at the class level. The fixed effects model and the disaggregated data model differ from the multilevel model with respect to $\operatorname{Var}\left(\hat{\beta}_{1}\right)$ but not necessarily with respect to $\hat{\beta}_{1}$ itself. The results for the aggregated data model correspond to those of the multilevel model if weighting of class $j$ is done by the factor $w_{\mathrm{R}}=\left(\sigma_{e}^{2} / n_{1 j}+\sigma_{u}^{2}\right)^{-1 / C}$ for Design 2, where $\mathrm{C}=\sum_{k x_{k}=x_{j}}\left(\sigma_{e}^{2} / n_{1 k}+\sigma_{y j}^{2}\right)^{-1}$, and $w_{\mathrm{R}}=\left(\sigma_{e}^{2} / n_{1 j}+\sigma_{\mathrm{w}}^{2}\right)^{-1 / C}$ for Design 1 and assuming treatment by class interaction, where $\mathrm{C}=\sum_{j}\left(\sigma_{e}^{2} / n_{j}+\sigma_{w 1}^{2}\right)^{-1}$. However data aggregation with these weights is hardly a simple alternative to multilevel analysis if the variance components are unknown and have to be 
estimated. When $\sigma_{\varepsilon}^{2} / n_{1 j}$ is large compared with $\sigma_{z i}^{2}$ or $\sigma_{u 1}^{2}$, these weights are almost equal to $w_{n}=n_{\mathrm{y}} / \Sigma_{j} n_{1 j}$ and weighting is done according to the number of pupils per class. On the other hand if $\sigma_{e}^{2} / n_{1 j}$ is small these weights are almost equal to $w_{\mathrm{m}}=1 / n_{2}$ which implies that no weighting is done. The treatment effect estimator with weighting by $w_{\mathrm{R}}$ will be bounded by the estimated treatment effects with weighting by $w_{\mathrm{II}}$ and $w_{\mathrm{III}}$. It can be shown (Bloch and Moses, 1988) that an unweighted analysis is at most $12.5 \%$ less efficient than weighting by the proper weights $w_{\mathrm{R}}$ if $\sigma_{\mathrm{u}}^{2} \geq \sigma_{\mathrm{e}}^{2}\left(\left(\min n_{1, j}\right)^{-1}-2\left(\max n_{1 j}\right)^{-1}\right)$ within each treatment condition for class level randomization, or $\sigma_{u 1}^{2} \geq \sigma_{e}^{2}\left(\left(\min n_{1 j}\right)^{-1}-2\left(\max n_{1,}\right)^{-1}\right)$ overall for randomization at the pupil level. A sufficient but not necessary condition is $\left(\max n_{1 j}\right) /\left(\min n_{1 j}\right) \leq 2$ overall for pupil level randomization, or within each treatment condition for class level randomization. On the other hand, the weights $w_{\text {II }}$ are generally preferred when treating classes as fixed (Lin, 1999).

\section{Example: Analysis of TVSFP data}

To compare the traditional models with the multilevel model in the case of varying class sizes the TVSFP data, with restriction to the Los Angeles pupils in the media or no-treatment control group, were analyzed. In the analyses two levels of nesting are taken into account: pupils within classes. Class sizes ranged from 1 till 27 with a mean of 12 pupils per class. All pupils within a class received the same treatment condition and the interaction between treatment condition and class cannot be estimated. Treatment condition was used as the only explanatory variable to model the outcome THKS, leaving the inclusion of the pretreatment THKS to Section 2.7.2.

The results of the analyses are presented in the upper half of Table 2.6. Compared with the multilevel model, the fixed effects model and the disaggregated data model both produce too large estimates of the treatment effect and too small standard errors. As a result test statistics are too large and $p$-values are too small, which was also true for non-varying class sizes (see Section 2.6). Furthermore the estimated treatment effect of the multilevel model is bounded by those of the aggregated data models with weighting according to cluster size and without weighting. The latter even produces an estimated treatment effect below zero which in this case is a result of not taking class sizes into account. Weighting according to class size results in a standard error which is smaller than that for the multilevel model due to the fact that this type of weighting ignores intra class correlation. The estimated treatment effect of the fixed effects model corresponds to that of the disaggregated data model and the aggregated data model with weighting according to class size since the dummy variables of the fixed effects model are coded such that they are orthogonal to treatment $x_{j}$. 
Table 2.6. Results of multilevel and traditional analyses of TVSFP data

Model

\begin{tabular}{|c|c|c|c|}
\hline Mult & Fixed effects & $\begin{array}{l}\text { Disaggregated } \\
\text { data }\end{array}$ & $\begin{array}{l}\text { Aggregated data Aggregated data } \\
\text { weighting by } w_{11} \text { no weighting }\left(w_{m}\right)\end{array}$ \\
\hline
\end{tabular}

Model without pre-test THKS

$\begin{array}{llllll}\hat{\sigma}_{u}^{2} & 0.166 & - & - & - & - \\ \hat{\sigma}_{e}^{2} & 1.718 & 1.708 & - & - & - \\ \hat{\sigma}_{r}^{2} & - & - & 1.871 & - & - \\ \hat{\beta}_{1}\left(\operatorname{SE}\left(\beta_{1}\right)\right) 0.056(0.070) & 0.089(0.045) & 0.089(0.047) & 0.089(0.067) & -0.041(0.082) \\ t_{\beta_{1}}(d f) & 0.8011(68) & 1.964(767) & 1.876(835) & 1.331(68) & -0.498(68) \\ p \text {-value } & 0.426 & 0.050 & 0.061 & 0.188 & 0.620\end{array}$

Model with pre-test THKS

\begin{tabular}{llllll}
$\hat{\sigma}_{u}^{2}$ & 0.107 & - & - & - & - \\
$\hat{\sigma}_{e}^{2}$ & 1.573 & 1.557 & - & - & - \\
$\hat{\sigma}_{r}^{2}$ & - & - & 1.674 & - & - \\
$\hat{\beta}_{1}\left(\hat{S E}\left(\hat{\beta}_{1}\right)\right) 0.085(0.061)$ & $0.138(0.046)$ & $0.106(0.045)$ & $0.106(0.060)$ & $-0.018(0.079)$ \\
$t_{\beta_{1}}(d f)$ & $1.340(66)$ & $2.966(766)$ & $2.369(833)$ & $1.727(67)$ & $-0.229(67)$ \\
$p$-value & 0.168 & 0.003 & 0.018 & 0.082 & 0.819 \\
\hline
\end{tabular}

\subsubsection{Models with covariates}

A covariate $c_{i f}$ may be split into a component $\bar{c}_{j}$ which varies only between classes, and a component $c_{i j}-\bar{c}_{\ell}$ which waries only within classes (Neuhaus and Kalbfleisch, 1998). These two components may then be added to the multilevel model (2.2), the fixed effects model (2.9), the disaggregated data model (2.10), and the aggregated data models (2.12) and (2.14). Then it can be shown that the $\operatorname{Var}\left(\hat{\beta}_{1}\right)$ given in Table 2.6 have to be divided by $\left(1-R^{2}\right)$, where $R^{2}$ is the squared multiple correlation between treatment condition and the other independent variables in the model, i.e. the two components of the covariate, and for the fixed effects model the dumny variables as well.

For randomization at the class level $\left(1-R^{2}\right)$ is equal to $\left(1-r_{x_{f}}^{2} \bar{c}\right)$ for the multilevel model, the disaggregated data model, and the aggregated data model, and the comparison for these models made in the previous section will roughly hold. Since there is multicollinearity between treatment condition, the $n_{2}-2$ dummy variables, and the class component of the covariate $\bar{c}_{j}$, the latter cannot be added to the fixed effects model. As the dummy variables 
(which have been centered) and the pupil component of the covariate $c_{i j}-\bar{c}_{j}$ are orthogonal to the treatment effect, $\left(1-R^{2}\right)$ is equal to 1 for the fixed effects model.

For randomization at the pupil level and assuming no treatment by class interaction, the (1-R $\left.{ }^{2}\right)$ equals $\left(1-r_{x_{i j}}^{2} c_{i j}-c_{j}\right)$ for the multilevel model and the disaggregated data model, and the comparison for these two models as made in Section 2.5 will still hold. Since there is multicollinearity between the $n_{2}-1$ dummy variables, and the class component of the covariate $\bar{c}_{j}$, the latter cannot be added to the fixed effects model. As the dummy variables are orthogonal to the treatment effect, $\left(1-R^{2}\right)$ is the same as for the multilevel model. The pupil level component $c_{i j}-\bar{c}_{j}$ of the covariate will is equal to zero in the aggregated data model, so the pupil level variance for the aggregated data model will be larger than for the other models, but also will the term $\left(1-\mathrm{R}^{2}\right)$ be equal to zero for the aggregated data model. Assuming treatment by class interaction, however, the formula for the $\operatorname{Var}\left(\hat{\beta}_{1}\right)$ in the presence of covariates will become more complex and are beyond the scope of this paper.

\section{Example: Analysis of TVSFP data (continued)}

The pre-treatment THKS was split into a component which varies at the class level and one which varies at the pupil level, and both components were added to the model as covariates. As a result, the estimated variance components at both levels will decrease. The results of the analyses are given in the lower part of Table 2.6. Observed $p$-values were too low for the fixed effects model, the disaggregated data model and the aggregated data model with weighting according to class size, leading to an incorrect rejection of the null hypothesis. Note that the treatment effect estimate according to the fixed effects model differs from the estimate by the disaggregated data model although the dummy variables are orthogonal to the treatment factor. This is due to the fact that both dummy variables and treatment factor slightly correlate with the class level covariate. The estimated treatment effect of the disaggregated data model corresponds to that of the aggregated data model with weighting according to class size, whereas the estimated treatment effect for the multilevel model is bounded by those of the aggregated data model with and without weighting.

\subsection{Discussion and conclusions}

In this study four regression models for the analysis of multilevel experimental data were compared: the multilevel model, the fixed effects model, the disaggregated data model, and the aggregated data model. To show the similarities with familliar ANOVA models, these models were also presented in terms of ANOVA notation. It was assumed that the conditions for random sampling of clusters from a larger population of clusters were satisfied, so that the experimental results were not only valid for the clusters involved in the study, but could also be generalized to the population of clusters. In that case the multillevel model should be used for the data analysis, but as this model is relatively new and rather complex, it was 
investigated whether the fixed effects model, the disaggregated data model, and the aggregated data model could be used as an altemative to the multilevel model. As criterion for the comparison the estimator of the treatment effect $\hat{\beta}_{1}$ and its variance $\operatorname{Var}\left(\hat{\beta}_{1}\right)$ were used, since these are generally of main interest in such experimental evaluations of treatments.

Randomization should be done at the person level if interaction between treatment condition and cluster is to be evaluated, and results in more efficient estimates of the treatment effect whether interaction is present or not. Analysis of data should then be done using the multilevel model in (2.2). This corresponds to a paired samples $t$-test after data aggregation for the case of equal cluster sizes and models without covariates, and this simple approach may thus be used as an alternative to the multilevel model. The treatment effect is correctly estimated by the fixed effects model and the disaggregated data model in the case of equal cluster sizes and models without covariates, but these two models generally underestimate its variance and so the null hypothesis of no treatment effect will be rejected too often if clusters are considered random and the results of the study have to be generalized to a larger population of clusters. Data aggregation is not a simple alternative to the multilevel model for varying cluster sizes since the weights are then a function of the variance components which are generally unknown, but it can be shown that an unweighted analysis is at most $12.5 \%$ less efficient if the maximum cluster size is at most twice the minimum cluster size for all the values of the variance components.

For person level randomization and no interaction between treatment condition and cluster, the fixed effects model may be used as an altemative to the multilevel model at least if no covariates are included in the model. The disaggregated data model slightly overestimates the variance of the treatment effect. So, contrary to the widespread belief the use of the disaggregated data model does not always result in an inflated type I error rate. Furthermore, the paired samples $t$-test always assumes treatment by cluster interaction and is less efficient than multilevel analysis or the use of the fixed effects model.

For randomization at the cluster level data aggregation followed by the independent samples $t$-test is equivalent to the multilevel model if cluster sizes do not vary and there are no covariates. The variance components have to be known to compute the proper weights for an aggregated analysis when cluster sizes vary, but an unweighted analysis may be useful if within both treatment conditions the maximum cluster size is at most twice the minimum cluster size. Furthermore, the fixed effects model and disaggregated data model will underestimate the variance of the treatment effect estimator, leading to an inflation of the type I error rate if clusters are assumed to be random. 


\section{References}

Aitkin, M., \& Longford, N. (1986). Statistical modelling issues in school effectiveness studies, Journal of the Royal Statistical Saciety, Series A, 149, 1-43.

Barcikowski, R. S. (1981). Statistical power with group mean as the unit of analysis, Journal of Educational Statistics, 6, 267-285.

Bass, M. J., McWinney, I. R., \& Donner, A. (1986). Do family physicians need medical assistance to detect and manage hypertension?, Canadian Medical Association Joumal, 134, 1247-1255.

Bloch, D. A., \& Moses, L. E. (1988). Nonoptimally weighted least squares, The American Statistician, 42, 50-53.

Bryk, A. S., \& Raudenbush, S. W. (1992). Hierarchical linear models. Newbury Park: Sage Publications.

Burton, P., Gurrin, L., \& Sly, P. (1998). Extending the simple linear regression model to account for correlated responses: An introduction to generalized estimating equations and multi-level mixed modelling, Statistics in Medicine, 17, 1261-1291.

Dunlop, D. D. (1994). Regression for longitudinal data: A bridge from least squares regression, The American Statistician, 48, 299-303.

Flay, B. R., Brannon, B. R., Johnson, C. A., Hansen, W. B., Ulene, A. L., Whitney- Santiel, D. A., Gleason, L. R., Sussman, S., Gavin, M. D., Glowacz, K. M., Sobol, D. F. \& Spiegel, D. C. (1988). The television school and family smoking prevention and cessation project. $\mathbb{I}$. Theoretical basis and program development, Preventive Medicine, 17, 585-607.

Flay, B. R., Miller, T. Q., Hedeker, D., Siddiqui, O., Britton, C. F., Brannon, B. R., Johnson, C. A.; Hansen, W. B., Sussman, S., \& Dent C. (1995). The television, school, and family smoking prevention and cessation project. VIII. Student outcomes and mediating variables, Preventive Medicine, 24, $29-40$.

Goldstein, H. (1986). Multilevel mixed linear model analysis using iterative generalized least squares, Biometrika, 73, 43-56.

Goldstein, H. (1989). Restricted unbiased iterative generalized least squares estimation, Biometrika, $76,622-623$.

Goldstein, H. (1995). Multilevel statistical models (2nd ed.). London: Edward Amold.

Gould, A. L. (1998), Multi-centre trial analysis revisited, Statistics in Medicine, 17, 1779-1797

Hartley, H. O., \& Rao, J. N. K. (1967). Maximum likelihood estimation for the mixed analysis of variance model, Biometrika, 54, 93-108.

Hedeker, D., Gibbons, R. D., \& Davis, J. M. (1991). Random regression models for multicenter clinical trials data, Psychopharmacology Bulletin, 27, 73-77.

Hedeker, D., McMahon, S. D., Jason, L. A., \& Salina, D. (1994). Analysis of clustered data in community psychology: With an example from a worksite smoking cessation project, American Journal of Community Psychology, 22, 595-615.

Hopkins, K. D. (1982). The unit of analysis: group means versus individual observations, American Educational Research Joumal, 19, 5-18.

Hox, J. J. (1994). Applied multilevel analysis. Amsterdam: TT-Publikaties.

Jones, B., Teather, J. W., \& Lewis, J. A. (1998). A comparison of various estimator of treatment difference for a multi-centre clinical trial, Statistics in Medicine, 17, 1767-1777.

Kreft, I., \& De Leeuw, J. (1998). Introducing multilevel modelling. London: Sage Publications.

Lin, Z. (1999). An issue of statistical analysis in controlled multi-centre studies: how shall we weight the centres?, Statistics in Medicine, 18, 365-373.

Longford, N. T. (1995). Random coefficient models. Oxford: Clarendon Press.

Moerbeek, M., Van Breukelen, G. J. P., \& Berger, M. P. F. (in press). Design issues for experiments in multilevel populations, Journal of Educational and Behavioral Statistics.

Neuhaus, J. M., \& Kalbfleiseh, J. D. (1998). Between- and within-cluster covariate effects in the 
anallysis of clustered data, Biometrics, 54, 638-645.

Parzen, M., Lipsitz, S. R. \& Dear, K. B. G. (1998). Does clustering affect the usual test statistics of no treatment effect in a randomized clinical trial?, Biometrical Journal, 40, 385-402.

Patterson, H. D., \& Thompson, R. (1971). Maximum likelihood estimation of components of variance. In $\mathbb{L} . \mathrm{C}, \mathrm{A}$. Corsten, \& T. Postelnicu (Eds.), Proceedings of the $8^{\text {th }}$ international biometric conference, Editura Academica Republicii Socialite România, Bucureşti, pp. 197207.

Raudenbush, S. W. (1993). Hierarchical linear models and experimental design. In L. K. Edwards (Ed), Applied analysis of variance in behavioral science (pp. 459-496). New York: Marcel Dekker.

Searle, S. R., Casella, G., \& McCulloch, C. E. (1992). Variance components. New York: John Wiley \& Sons.

Senn, S. (1998). Some controversies in planning and analysing multi-centre trials, Statistics in Medicine, 17, 1753-1765.

Snijders, T. A. B., \& Bosker, R. J. (1999). Multilevel analysis: An introduction to basic and advanced multilevel modelling. London: Sage Publications.

Sommer, A., Tarwotjo, I., Djunaedi, E., West, K. P., Loeden, A. A., Tilden, R., \& Mele, L. (1986). Impact of vitamin A supplementation on childhood mortality. A randomised controlled community trial, Lancet, 1, 1169-1173.

Sullivan, L. M., Dukes, K. A. and Losina, E. (1999). An introduction to hierarchical linear modelling, Statistics in Medicine, 18, 855-888. 


\title{
Chapter 3
}

\section{Optimal experimental designs for linear multilevel models}

\begin{abstract}
For the design of experiments in multilevel populations the following questions may arise: what is the optimal level of randomization, what is the optimal allocation of units, given a certain budget for sampling and measuring, and what is the required budget for obtaining a certain power of the test of no treatment effeet? In this article these questions will be dealt with for populations with two or three levels of nesting and continuous outcomes. Multilevel models are used to model the relationship between experimental condition and the outcome variable. The estimator of the regression coefficient associated with treatment condition is of main interest and should be estimated as efficiently as possible. Therefore, its variance is used as a criterion for optimizing the level of randomization and the allocation of units.
\end{abstract}

Key words: multilevel models, experimental designs, level of randomization, allocation of units, power

Acknowledgments: We wish to thank Ton Ambergen for checking the formulas in Table 3.2 This paper has been accepted for publication in Journal of Educational and Behavioral Statistics (in reduced form and under the tifle 'Design issues for experiments in multilevel populations'). 


\subsection{Introduction}

In many sciences, experiments are carried out to assess the effect of different treatments on outcome variables of individuals. In such experiments generally two treatment conditions are compared, for example an intervention and a control. The aim of the experiment is to estimate and test the between-group difference in outcomes. In health sciences, for example, experiments can be carried out to assess the effect of a newly developed smoking prevention program relative to a control on smoking behavior of adolescents. In medical sciences, the effect of a new medicine relative to an old medicine on the recovery of patients may be assessed. In educational sciences, the effect of a new curriculum on the math achievement of pupils may be established by comparing it with an old curriculum. When individuals are not nested within cllusters, an important design issue concerns the required number of observations in each treatment group in order to achieve a certain power for the test on treatment effect. The formula for this number of experimental units can be found in, for example, Hsieh (1988). In practice, however, populations often have a multilevel structure with individuals nested within clusters which may themselves be nested within higher order clusters. In the smoking prevention example, adolescents might be nested within schools or neighborhoods. For such experiments three design issues may arise.

The first issue concerns the optimal level of randomization to treatment conditions. In principle, randomization can be done at any level of the multilevel data structure. For example, in smoking prevention interventions with pupils nested within classes and classes nested within schools, we may choose to randomize pupils, classes or even entire schools to treatment conditions. Although the purpose of a treatment is mostly to produce an effect on individuals, in practice often entire clusters are randomized to treatment conditions. Reasons for randomization at the cluster level are ethical concerns, political and administrative factors, and the need to reduce costs and treatment group contamination, which occurs when information leaks from the intervention group to the control group (e.g., Donner, Brown and Brasher, 1990). Apart from these, in community-based interventions, in which the intervention will necessarily affect all members of a cluster, there is no alternative to cluster randomization (e.g., Gail, Mark, Carroll, Green and Pee, 1996).

The second design issue concerns the optimal allocation of units, that is the optimal sample sizes at each level of the multilevel data structure, given the budget for sampling and measuring units and the level of randomization. Is it preferable to sample a few clusters with a lot of individuals per cluster, or to sample many clusters with just a few individuals per cluster? In practice, a design with few clusters and many individuals per cluster is easier to obtain than a design with many clusters and a small number of individuals per cluster, because sampling in an already selected cluster may be less expensive than sampling in a new cluster, and often only a few clusters are willing to participate in a study. On the other hand the number of individuals to be sampled within a cluster can, of course, not be larger than the total number of individuals in that cluster. 
The third design issue is related to the second and concems the required budget in order to obtain a certain power of the test of no treatment effect, given the level of randomization.

So far, little statistical research on these three design issues has been done; see Goldstein (1995), Section 11.2. Donner, Birkett and Buck (1981) and Hsieh (1988) showed that randomization at the cluster level is less efficient in terms of estimator variance than randomization at the individual level and thus tests on treatment effect have less power to detect a real intervention effect. Raudenbush (1997) derived optimal allocations of units for cluster randomized designs with and without covariates and compared the efficiency of these two designs. Snijders and Bosker (1993) derived optimal allocations of units for two level designs with any number of explanatory variables at both levels. Mok (1996) compared two level designs in which the number of individuals per cluster is larger than, equal to, or smaller than the number of clusters by means of simulation studies. She based the comparisons on bias, sampling variance, and MSE of the parameter estimates. Domner, Birkett and Buck (1981) and Hsieh (1988) give sample size formulas for obtaining a certain power of the test concerning treatment effect for experiments in populations with two levels of nesting and with randomization to treatment conditions at the cluster level.

These results will be expanded in this study and the three design issues will be dealt with for populations with two and three levels of nesting and for continuous outcomes. The linear multilevel regression model is used to describe the relation between treatment condition and outcome. Although covariates may be included in the models, in experimental designs the regression coefficient associated with treatment condition is generally of main interest and has to be estimated as efficiently as possible to achieve a maximum power of the test of treatment effect. The optimal level of randomization that minimizes its variance will be presented and the increase in variance if randomization is done at another level will be studied in terms of relative efficiency. Next, the optimal allocation of units at which the variance of this estimator is minimized will be established, given the budget for measuring and sampling and the level of randomization. This will be done for models with and without a random slope. Thereafter, the required budget to achieve a certain power of the test of the treatment effect will be given. Finally some conclusions and suggestions for further research will be given.

\subsection{Models and estimators}

To help understanding of the application of the methods in practice, an example is used throughout this paper, namely a smoking prevention intervention study with pupils nested within classes and classes nested within schools. Pupils are referred to as level one units, classes as level two units, and schools as level three units and we thus have three levels of nesting. Ignoring the nesting of classes within schools results in two levels of nesting. 
Pupils are indexed by $i$, classes by $j$ and schools by $k$. Throughout this paper, a balanced design will be assumed; that is we have a sample of $n_{3}$ schools, $n_{2}$ classes per school and $n_{1}$ pupills per class. Furthermore, it will be assumed that the variances of random effects are known. The models that will be introduced in this section can be used for the comparison of the effects of two different treatments (the smoking prevention intervention and a control) on the outcome of smoking behavior, which is assumed to be measured on a continuous scale. Randomization to these two treatment conditions can be done at the school level, the class level, or the pupil level. If randomization is done at the pupil level, $\frac{1}{2} n_{1}$ pupils are randomized to the control group and $\frac{1}{2} n_{1}$ pupils are randomized to the intervention group within each class, and thus $n_{1}$ has to be even. Similarly, if randomization is done at the class level, the number of classes per school has to be even, and with randomization at the school level, the number of schools has to be even.

Our derivations will be based upon multilevel models (e.g., Goldstein, 1995), also referred to as hierarchical linear models (e.g., Bryk and Raudenbush, 1992), or random coefficient models (e.g., Longford, 1995). Multilevel models can be used when we have a random sample at each level of the multilevel data structure. In the smoking prevention intervention example, we have a sample of schools from the entire population of schools, a sample of classes within each sampled school, and a samplle of pupils within each sampled class. Since schools, classes and pupils are assumed to be sampled randomly, these have to be included as random effects in our model. Treatment condition and covariates, however, are included as fixed effects. So our model contains both fixed and random effects and is a mixed effect model.

In this paper, we deal with models without covariates (but see the end of this section). For three levels of nesting the model which relates smoking behavior, denoted by $y$, to treatment condition, denoted by $x$; is given by

$$
y_{i j k}=\beta_{0}+\beta_{1} x_{i j k}+v_{0 k}+u_{0 j k}+e_{0 i j k}
$$

where $v_{0 j}, u_{0 j k}$ and $e_{j i k}$ are random effects at the school $(k)$, class $(j)$, and pupil $(i)$ level, respectively. These random effects are independently distributed with zero mean and variances $\sigma_{w 0}^{2}, \sigma_{w 0}^{2}$, and $\sigma_{a}^{2}$, respectively. If randomization is done at the class level the $i$ can be left out of the subscript of $x$ in (3.1). Similarly, if randomization is done at the school level the $i$ and $j$ can be left out of the subscript of $x . \beta_{\mathbb{N}}$ is the regression coefficient associated with treatment condition and is considered to be fixed in this section and Section 3.3. In Section 3.4 some attention will be paid to optimal designs for models with random slopes.

For each level of randomization, the variance of a pupil's outcome, given the fixed part $\beta_{0}+\beta_{1} x_{i j k}$, consists of three components, the so-called variance components:

$$
\operatorname{Var}\left(y_{i j k}\right)=\sigma_{w 0}^{2}+\sigma^{2}+\sigma_{e}^{2}
$$


The outcomes of pupils $i$ and $i$ within the same class $j$ are dependent, and their covariance is:

$$
\operatorname{Cov}\left(y_{i j k} y_{i j k}\right)=\sigma_{w 0}^{2}+\sigma_{w 0}^{2} \text {, for } i \neq i \text {. }
$$

Similarly the dependency of outcomes of pupils $i$ and $i$ " within the same school $k$ but within different classes $j$ and $j$ ' is given by

$$
\operatorname{Cov}\left(y_{i j k^{2}} y_{i j^{\prime} k^{\prime}}\right)=\sigma_{w 0^{\prime}}^{2} \text { for } j \neq j^{\prime \prime} \text {. }
$$

With two levels of nesting the subscript $k$, the random effect $v_{0 k}$, and the variance component $\sigma_{v 0}^{2}$ can be left out of (3.1), (3.2) and (3.3), and (3.4) does not apply.

Since the outcomes are correlated and the variances of the random effects are assumed to be known, the generalized least squares estimator is used for the multilevel model in (3.1). For a three level design $\beta_{1}$ is estimated unbiasedly by

$$
\hat{\beta}_{1}=\frac{\sum_{i j k} x_{i j k} y_{i j k}}{n_{1} n_{2} n_{3}}=\frac{\bar{y}_{\ldots t}-\bar{y}_{\ldots c}}{2},
$$

in which $\bar{y}_{\ldots, t}$ and $\bar{y}_{\ldots e}$ are the mean responses in the intervention and control group, respectively. Formula (3.5) holds if treatment groups are coded by -1 and +1 . If they are coded by 0 and +1 , the estimator of $\beta_{1}$ is equal to $\bar{y}_{\ldots l} \bar{y}_{\ldots .}$. For a two level design, the subscript $k$ is left out and $n_{3}$ is set equal to unity. For both two and three levels of nesting and for each level of randomization the variance of the estimator associated with treatment condition, that is $\operatorname{Var}\left(\hat{\beta}_{1}\right)$, is given in Table 3.1. For the derivation we refer to the appendix. These variances only hold for treatment groups coded by -1 and +1 . If they are coded by 0 and +1 , the variances as given in Table 3.1 have to be multiplied by four. Note that $\operatorname{Var}\left(\hat{\beta}_{1}\right)$ for two levels of nesting and randomization at the class levell is a special case of Equation (37) in Snijders and Bosker (1993).

In our discussion we have not included covariates in our models. In general, however, the outcome variable smoking behavior will be influenced by covariates such as pretreatment smoking behavior or school type. It can be shown that if such covariates are grandmean centered and if their coefficients are fixed parameters, the results in Table 3.1 hold approximately, especially if the sample sizes at each level are not too small. This is due to the experimental (randomized) design, which ensures that treatment condition and covariate are uncorre]ated, apart from some sampling error (Moerbeek, Van Breukelen and Berger, 1999). Consequently, the results in the following sections also hold approximately. 
Table 3.I $\operatorname{Var}\left(\beta_{1}\right)$ for each Level of Randomization and Assuming a Fixed Slope

\begin{tabular}{lll}
\hline \hline Number of levels & Level of randomization & $\operatorname{Var}\left(\hat{\beta}_{1}\right)$ \\
\hline three & pupil & $\frac{\sigma_{e}^{2}}{n_{1} n_{2} n_{3}}$ \\
three & class & $\frac{n_{1} \sigma_{u 0}^{2}+\sigma_{e}^{2}}{n_{1} n_{2} n_{3}}$ \\
three & school & $\frac{n_{1} n_{2} \sigma_{w 0}^{2}+n_{1} \sigma_{u 0}^{2}+\sigma_{e}^{2}}{n_{1} n_{2} n_{3}}$ \\
two & pupil & $\frac{\sigma_{e}^{2}}{n_{1} n_{2}}$ \\
two & class & $\frac{n_{1} \sigma_{u 0}^{2}+\sigma_{e}^{2}}{n_{1} n_{2}}$ \\
\hline
\end{tabular}

Note. These variances hold if treatment conditions are coded -1 and +1 .

\subsection{Optimal experimental designs for the model with random intercept and} fixed slope

\subsubsection{Optimal level of randomization}

As written in the introduction, the optimal level of randomization is that level for which $\operatorname{Var}\left(\beta_{1}\right)$ is minimized. From Table 3.1 it is clear that $\operatorname{Var}\left(\beta_{1}\right)$ decreases if randomization to treatment conditions is done at a lower level and thus the pupil level is the optimal level of randomization. Randonization at a higher level often leads to higher values for $\operatorname{Var}\left(\beta_{1}\right)$. This can be seen by considering the relative efficiency of $\hat{\beta}_{1}$. The relative efficiency of two unbiased estimators of $\beta_{1}$ is defined as the ratio of the reciprocal of their variances. For two levels of nesting

$$
\text { relative efficiency }=\frac{\sigma_{e}^{2}}{n_{1} \sigma_{t+0}^{2}+\sigma_{e}^{2}}=\frac{1-\rho}{\left(n_{1}-1\right) p+1},
$$

where the $\operatorname{Var}\left(\beta_{1}\right)$ for randomization at the class level is related to $\operatorname{Var}\left(\beta_{1}\right)$ for randomization at the pupil level. The intra-class correlation coefficient $\rho$ measures the amount of variance between classes, that is $\rho=\sigma_{u 0}^{2} /\left(\sigma_{u 0}^{2}+\sigma_{e}^{2}\right)$. The inverse of the relative efficiency gives the number of times the design for randomization at the class level needs to be replicated to be as efficient as randomization at the pupil level. In Figure 3.1 the relative efficiency is plotted as a function of $n_{1}$ and the intra-class correlation coefficient. Figure 3.1 shows that if $\rho$ or $n_{1}$ 
increase, the relative efficiency decreases. For low values of $\rho$ the relative efficiency is already considerably small. For $p=0.1$ it is equal to $0.47,0.31$, and 0.18 , for $n_{1}=10, m_{1}=20$, and $n_{1}=40$, respectively. When $\rho$ approaches unity the relative efficiency goes to zero. Similarly, it can be inferred from Table 3.1 that for three levels of nesting the relative efficiency of $\hat{\beta}_{1}$ obtained with randomization at the pupil level versus randomization at the school level decreases with $n_{1}, n_{2}$, the intra-class correlation coefficient, and the intra-school correlation coefficient, which measures the amount of variance between schools.

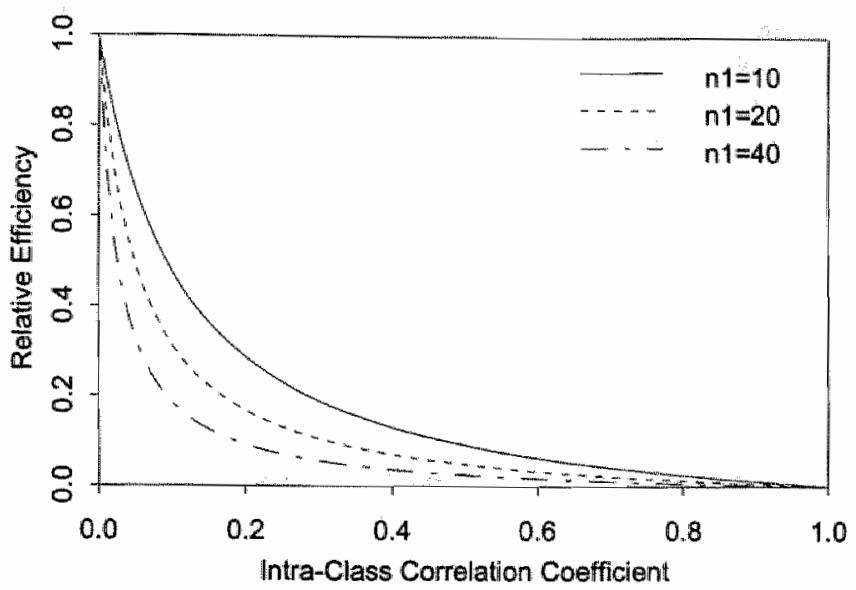

Figure 3.1. Relative efficiency of $\hat{\beta}_{1}$ for randomization at the class level versus randomization at the pupil level as a function of the intra-class correlation coefficient and the number of pupils per class.

\subsubsection{Optimal allocation of units}

Although the lowest level of the multilevel data structure is the optimal level of randomization, randomization at this level is not always possible. Therefore, the optimal allocation of units will be derived for each level of randomization.

As written in the introduction, the optimal allocation of units consists of those values $n_{1}, n_{2}$, and $n_{3}$ for which $\operatorname{Var}\left(\beta_{1}\right)$ is minimized. Optimal allocations are now derived under the condition that the budget for sampling and measuring units is fixed to $C$. We require to

$$
\text { minimize } \operatorname{Var}\left(\hat{\beta}_{1}\right)
$$

subject to the condition 


$$
C=c_{1} n_{1} n_{2} n_{3}+c_{2} n_{2} n_{3}+c_{3} n_{3} \quad\left(c_{l}>0, n_{l} \geq 2 \text { for } l=1,2,3\right)
$$

for three levels of nesting, or to the condition

$$
C=c_{1} n_{1} n_{2}+c_{2} n_{2} \quad\left(c_{l}>0, n_{l} \geq 2 \text { for } l=1,2\right)
$$

for two levels. Here $c_{3}$ are the costs for sampling and measuring a school, $c_{2}$ are the costs for sampling and measuring a class in an already sampled school, and $c_{1}$ are the costs for sampling and measuring a pupil in an already sampled class. $c_{3}$ may vary across schools, for instance because of different travel times. However, at the time of planning the experiment, it is not known which schools will be sampled. Therefore an average value of $c_{3}$ has to be used for planning the experiment. In this article $C, c_{\|}, c_{2}$, and $c_{3}$ are the budget and costs in dollars, but of course any other currency can be used. We restrict $n_{1}, n_{2}$, and $n_{3}$ to be at least two in order to obtain a multilevel data structure, and to be able to estimate the variance components at the class and school level. For three levels of nesting the optimal $n_{l}$ can be obtained by

Table 3.2 Optimal Allocation of Units for each Level of Randomization and Assuming a Fixed Slope

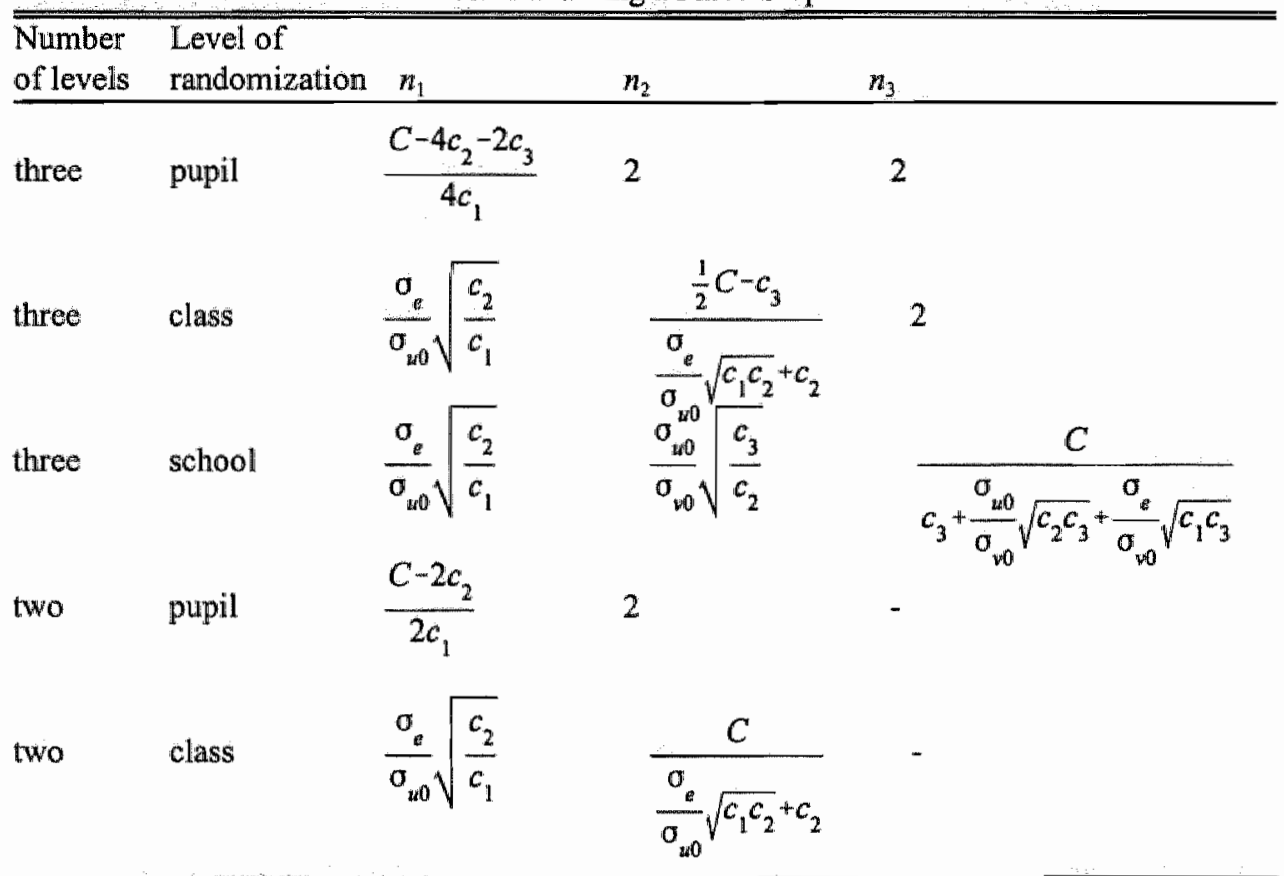

Note. For two level designs the nesting of classes within schools is omitted and thus the optimal $n_{3}$ is not given. Treatment conditions are coded -1 and +1 . 
expressing either $n_{1}$, or $n_{2}$, or $n_{3}$ in terms of the other two $n_{l}$ and the costs using (3.8), substituting this expression into the formula for $\operatorname{Var}\left(\beta_{1}\right)$ as given in Table 3.1 , and solving for the other two $n_{f}$. For two levels of nesting, the procedure is comparable. The optimal values of the $n_{l}$ thus obtained, and the minimum $\operatorname{Var}\left(\hat{\beta}_{1}\right)$ under the optimal allocation are given in Table 3.2 and Table 3.3, respectively. A lower $\operatorname{Var}\left(\beta_{1}\right)$ can be obtained by using a higher budget $C$, which results in sampling more units only at the level at which randomization is done since the sample sizes at the other levels are independent of $C$, see Table 3.2. For two levels of nesting with randomization at the class level, the optimal allocation corresponds with equation (39) in Snijders and Bosker (1993). Note that the values of $n_{l}$ other than 2 in Table 3.2 are obtained by treating the $n_{l}$ as continuous variables, while actually they are integers. Thus the values of the $n$ 's obtained with the formulas in Table 3.2 meed to be rounded off to the nearest integer in such a way that the budget is not exceeded; and $\operatorname{Var}\left(\hat{\beta}_{1}\right)$ may somewhat exceed the minimum value given by Table 3.3 . In the next section an example will illustrate these results.

Table 3.3 $\operatorname{Var}\left(\hat{\beta}_{1}\right)$ under the Optimal Allocation of Units for each Level of Randomization and Assuming a Fixed Slope

\begin{tabular}{|c|c|c|}
\hline Number of levels & Level of randomization & $\operatorname{Var}\left(\hat{\beta}_{1}\right)$ \\
\hline three & pupil & $\frac{\sigma^{2} c_{1}}{C-4 c_{2}-2 c_{3}}$ \\
\hline three & class & $\frac{\left(o_{40} c_{2}+a_{d} c_{1}\right)}{C-2 c_{3}}$ \\
\hline three & school & 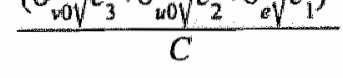 \\
\hline two & pupil & $\frac{\sigma_{e}^{2} c_{1}}{C-2 c_{2}}$ \\
\hline two & class & $\frac{\left(w 0 V^{2} e V^{2}\right.}{C}$ \\
\hline
\end{tabular}

Note. Treatment conditions are coded -1 and +1 . 


\subsubsection{An example}

In a multilevel design the costs of sampling and measuring at a higher level are often larger than the costs of sampling and measuring at a lower level. Furthermore, there is often more variation between lower level units than between higher level units. The following set of values is an example of these cases: $c_{1}=\$ 100, c_{2}=\$ 200, c_{3}=\$ 300, C=\$ 20000, \sigma_{e}^{2}=16$, $\sigma_{u 0}^{2}=2$, and $\sigma_{v 0}^{2}=0.5$. For these values the optimal allocation of units can be calculated from Table 3.2 and is shown in Figures 3.2, 3.3, and 3.4.

In Figure 3.2 contour lines of $\operatorname{Var}\left(\hat{\beta}_{1}\right)$ for randomization at the pupil level are plotted in the $\left(n_{1}, n_{2}\right)$ plane for the following values of $\operatorname{Var}\left(\beta_{1}\right): 0.09,0.095,0.10,0.15,0.11$. The lines $n_{1}=2, n_{2}=2$ and $n_{3}=2$ are shown as dotted lines. In the area enclosed by these three dotted lines the conditions given in (3.8) are satisfied; thus the optimal allocation of units under these conditions is located within this area. Clearly, $\operatorname{Var}\left(\beta_{1}\right)$ decreases as $n_{1}$ increases, and the optimal allocation is given by point $A$ in Figure 3.2, where $n_{2}=n_{3}=2, n_{1}=46.5$, and $\operatorname{Var}\left(\beta_{1}\right)=0.086$ from Table 3.3. We take $n_{1}=46$ as it has to be an integer, giving the costs are $c_{1} n_{1} n_{2} n_{3}+c_{2} n_{2} n_{3}+c_{3} n_{3}=\$ 19800$ and $\operatorname{Var}\left(\hat{\beta}_{1}\right)=0.087$, virtually unchanged. However, a value of 46 pupils per class may be too large for practical purposes and different allocations of units may be more realistic. In this example the minimum is a flat one, and so certain other allocations of units for which the budget is not exceeded will give much the same values for $\operatorname{Var}\left(\beta_{1}\right)$. For example, if $n_{1}=21, n_{2}=2, n_{3}=4$, then the costs are $\$ 19600$ and $\operatorname{Var}\left(\hat{\beta}_{1}\right)=0.095$; while if $n_{1}=10, n_{2}=8, n_{3}=2$, then the costs are $\$ 19800$ and $\operatorname{Var}\left(\hat{\beta}_{1}\right)=$ 0.100 . If the difference between a value such as 0.095 or 0.100 and the formal minimum 0.087 is not worth bothering, these allocations may be considered as an alternative to the formal optimum $(46,2,2)$.

In Figure 3.3 contour lines of $\operatorname{Var}\left(\beta_{1}\right)$ for randomization at the class level are plotted for $\operatorname{Var}\left(\hat{\beta}_{\|}\right)=0.19,0.20,0.21,0.22$, and 0.23 . Again, the conditions given in (3.8) are satisfied in the area enclosed by the dotted lines. Figure 3.3 shows that $\operatorname{Var}\left(\hat{\beta}_{1}\right)$ decreases as $n_{1}$ increases and then increases; while for given $n_{1}, \operatorname{Var}\left(\beta_{1}\right)$ decreases as $n_{2}$ increases. The optimal allocation is given by point $\mathrm{A}$ where $n_{1}=4, n_{2}=16.2, n_{3}=2$, and $\operatorname{Var}\left(\beta_{1}\right)=0.186$, as follows from Table 3.2 and Table 3.3. Rounding off to $n_{2}=16$ gives costs $c_{1} n_{1} n_{2} n_{3}+c_{2} n_{2} n_{3}+c_{3} n_{3}=\$ 19800$ and $\operatorname{Var}\left(\beta_{1}\right)$ increases somewhat to 0.188 .

For randomization at the school level, contour lines are plotted in Figure 3.4 for the following values of $\operatorname{Var}\left(\beta_{1}\right): 0.27,0.30,0.33$, and 0.36 . The optimal allocation of units is given by point $\mathrm{A}$ where $n_{1}=4, n_{2}=2.4, n_{3}=11.3$, and $\operatorname{Var}\left(\hat{\beta}_{1}\right)=0.261$, see Table 3.2 and Table 3.3. As $n_{1}, n_{2}$, and $n_{3}$ have to be integers, we take $n_{2}=2$ and $n_{3}=12$ for which the costs are $c_{1} n_{1} n_{2} n_{3}+c_{2} n_{2} n_{3}+c_{3} n_{3}=\$ 18000$ and $\operatorname{Var}\left(\hat{\beta}_{1}\right)=0.292$. However, a lower $\operatorname{Var}\left(\beta_{1}\right)$ can be obtained with $n_{1}=6, n_{2}=2$ and $n_{3}=10$, giving costs of $\$ 19000$ and $\operatorname{Var}\left(\beta_{1}\right)=0.283$. 


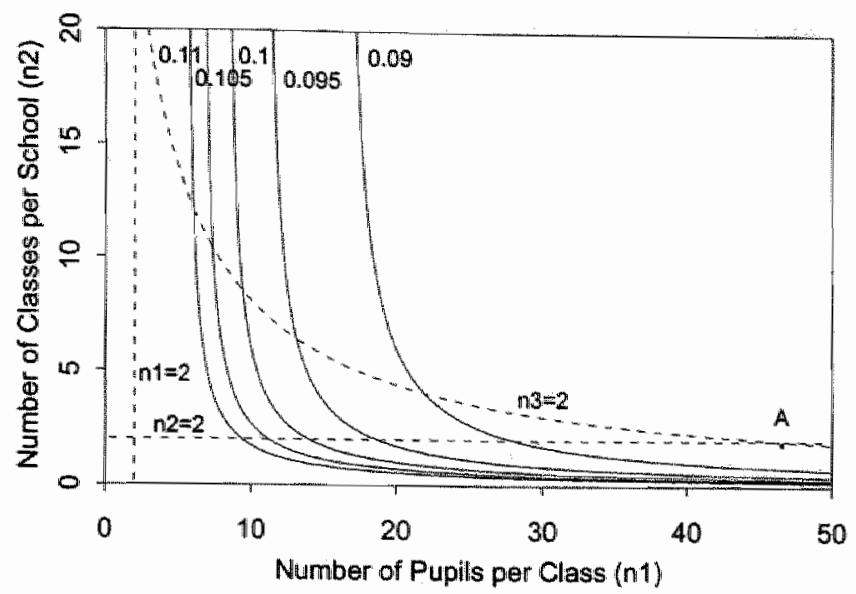

Figure 3.2. Preconditions (dashed lines) and contour lines for $\operatorname{Var}\left(\beta_{1}\right)$ (solid lines) in the $\left(n_{1}, n_{2}\right)$-plane. Three levels of nesting, randomization at the pupil level, and $c_{1}=\$ 100$, $c_{2}=\$ 200, c_{3}=\$ 300, C=\$ 20000, \sigma_{e}^{2}=16, \sigma_{u 0}^{2}=2, \sigma_{v 0}^{2}=0.5$.

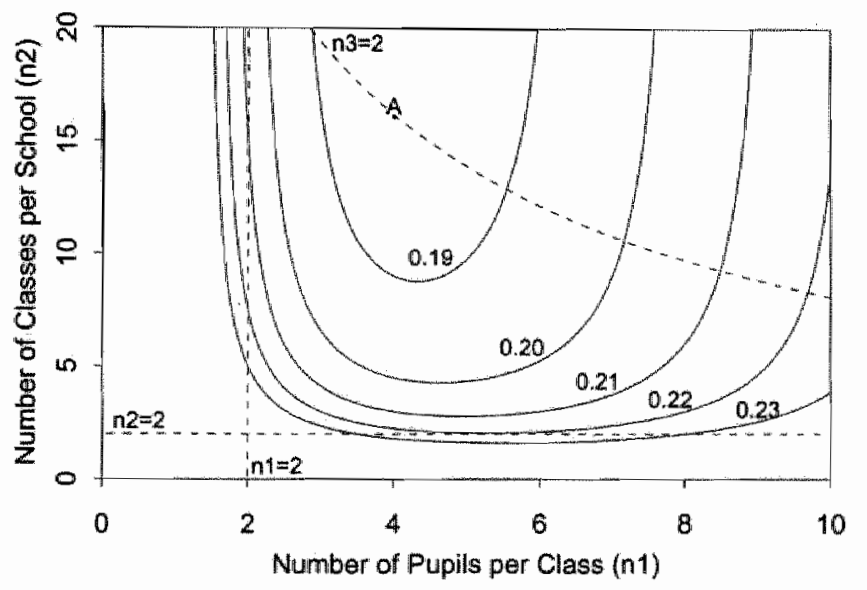

Figure 3.3. Preconditions (dashed lines) and contour lines for $\operatorname{Var}\left(\beta_{1}\right)$ (solid lines) in the $\left(n_{1}, n_{2}\right)$-plane. Three levels of nesting, randomization at the class level, and $c_{1}=\$ 100$, $c_{2}=\$ 200, c_{3}=\$ 300, C=\$ 20000, \sigma_{e}^{2}=16, \sigma_{u 0}^{2}=2, \sigma_{v 0}^{2}=0.5$. 


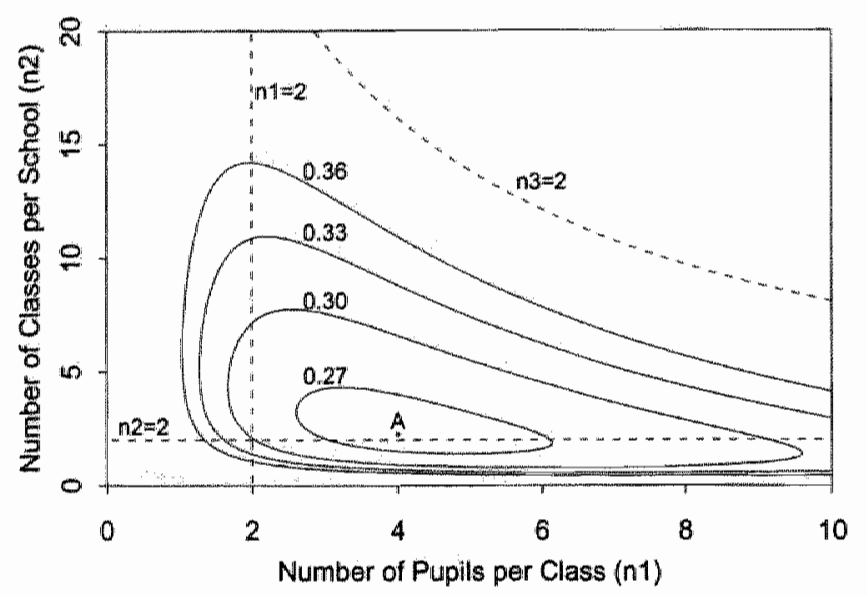

Figure 3.4. Preconditions (dashed lines) and contour lines for $\operatorname{Var}\left(\hat{\beta}_{1}\right)$ (solid lines) in the $\left(n_{1}, n_{2}\right)$-plane. Three levels of nesting, randomization at the school level, and $c_{1}=\$ 100$, $c_{2}=\$ 200, c_{3}=\$ 300, C=\$ 20000, \sigma_{e}^{2}=16, \sigma_{t 0}^{2}=2, \sigma_{N 0}^{2}=0.5$.

\subsubsection{Minimizing the budget}

So far the allocation of units for minimizing $\operatorname{Var}\left(\beta_{1}\right)$ under the condition that the budget for measuring and sampling is fixed to a certain value, was derived. On the other hand, it may also be worthwhile to derive the allocation of units for minimizing the budget for measuring and sampling to obtain a certain value $\operatorname{Var}\left(\hat{\beta}_{1}\right)$. Again we have an optimization problem: the budget for measuring and sampling as given in (3.8) or (3.9) has to be minimized under the condition that $\operatorname{Var}\left(\hat{\beta}_{1}\right)$ as given in Table 3.1 is fixed. The solution to this problem can directly be derived from Table 3.2 and Table 3.3 by considering the following. If the budget for measuring and sampling is equal to $C$, the allocation of units as given in Table 3.2 results in minimal value of $\operatorname{Var}\left(\beta_{11}\right)$ as given in Table 3.3. So, this budget $C$ is also the minimal budget to obtain that particular value of $\operatorname{var}\left(\beta_{1}\right)$, since if there existed a smaller budget with other allocation yielding the same $\operatorname{Var}\left(\beta_{1}\right)$, then our allocation given $C$ would not be optimal. Thus if a value of $\operatorname{Var}\left(\beta_{1}\right)$ equal to $V$ is required, the minimal budget to obtain this $\operatorname{Var}\left(\beta_{1}\right)$ follows by setting $\operatorname{Var}\left(\hat{\beta}_{1}\right)$ as given in Table 3.3 equal to $V$ and solving for the budget $C$. The corresponding optimal allocation of units then follows from Table 3.2. For example, assume that randomization is done at the school level, $c_{1}=\$ 100, c_{2}=\$ 200$, $c_{3}=\$ 300, \sigma_{e}^{2}=16, \sigma_{w 0}^{2}=2$, and $\sigma_{v 0}^{2}=0.5$, and $\operatorname{Var}\left(\hat{\beta}_{1}\right)$ has to be equal to 0.2 . Then the budget has to be equal to $\$ 26100$ with corresponding optimal allocation (before rounding off) $n_{1}=4, n_{2}=2.4$, and $n_{3}=14.7$. 


\subsection{Optimal experimental designs for the model with random intercept and random slope}

We will now assume that there is an interaction between treatment condition and higher levels units (classes and schools). This can be achieved by including random slopes into the multilevel model. So for randomization at the pupil level we have

$$
y_{i j k}=\beta_{0}+\beta_{1} x_{i j k}+v_{0 k}+u_{0 j k}+v_{1 k} x_{i j k}+u_{1 j, k} x_{i j k}+e_{i j k}
$$

where $v_{0 k} \sim \mathrm{N}\left(0, \sigma_{v 0}^{2}\right)$ and $v_{i k} \sim \mathrm{N}\left(0, \sigma_{\mathrm{v} 1}^{2}\right)$ are random effects at the school level corresponding to the intercept and slope, respectively, and their covariance is denoted $\sigma_{v 01}$. Likewise, $u_{0 j k} \sim \mathrm{N}\left(0, \sigma_{u 0}^{2}\right)$ and $u_{j \mathrm{jk}} \sim \mathrm{N}\left(0, \sigma_{u 1}^{2}\right)$ are random effects at the class level corresponding to the intercept and slope, respectively, and their covariance is denoted $\sigma_{u 00}$. As in Section 3.3 $e_{i j k} \sim \mathrm{N}\left(0, \sigma_{e}^{2}\right)$ is the random effect at the pupil level. For randomization at the pupil level there are two treatment conditions per class and all variance components can be estimated. For randomization at the class level there is just one treatment condition per class and so $\sigma_{t 00}^{2}$ and $\sigma_{u 1}^{2}$ cannot be estimated separately. Instead their sum will be estimated which will be denoted $\sigma_{u}^{2}$. Similarly, there is only one treatment condition per school for randomization at the school level, and the sums $\sigma_{u}^{2}=\sigma_{u 0}^{2}+\sigma_{u 1}^{2}$ and $\sigma_{v}^{2}=\sigma_{v 0}^{2}+\sigma_{v 1}^{2}$ will be estimated.

Table 3.4 $\operatorname{Var}\left(\beta_{1}\right)$ for each Level of Randomization and Assuming a Random Slope

\begin{tabular}{lll}
\hline \hline Number of levels & Level of randomization & $\operatorname{Var}\left(\beta_{1}\right)$ \\
\hline three & pupil & $\frac{n_{1} n_{2} \sigma_{v 1}^{2}+n_{1} \sigma_{u 1}^{2}+\sigma_{e}^{2}}{n_{1} n_{2} n_{3}}$ \\
three & class & $\frac{n_{1} n_{2} \sigma_{v 1}^{2}+n_{1} \sigma_{u}^{2}+\sigma_{e}^{2}}{n_{1} n_{2} n_{3}}$ \\
three & school & $\frac{n_{1} n_{2} \sigma_{v}^{2}+n_{1} \sigma_{u}^{2}+\sigma_{u}^{2}}{n_{1} n_{2} n_{3}}$ \\
two & pupil & $\frac{n_{1} \sigma_{u 1}^{2}+\sigma_{e}^{2}}{n_{1} n_{2}}$ \\
& & $\frac{n_{1} \sigma_{u}^{2}+\sigma_{s}^{2}}{n_{1} n_{2}}$ \\
two & class &
\end{tabular}

Note. These variances hold if treatment conditions are coded -1 and +1 . $\sigma_{u}^{2}=\sigma_{u 0}^{2}+\sigma_{u 1}^{2}, \quad \sigma_{v}^{2}=\sigma_{v 0}^{2}+\sigma_{v 1}^{2}$ 


\subsubsection{Optimal level of randomization}

Table 3.4 shows the $\operatorname{Var}\left(\beta_{1}\right)$ for each level of randomization and for models with two and three levels of nesting. See the appendix for the derivations. From this table it is clear that the pupil level is still the optimal level of randomization. But since the variances corresponding to random slopes at higher levels are included in the $\operatorname{Var}\left(\beta_{1}\right)$ for randomization at the pupil level, the efficiency for randomization at the class or school level relative to randomization at the pupil will be higher for models with random slopes than for models without random slopes.

Table 3.5 Optimal Allocation of Units for each Level of Randomization and Assuming a Random Slope

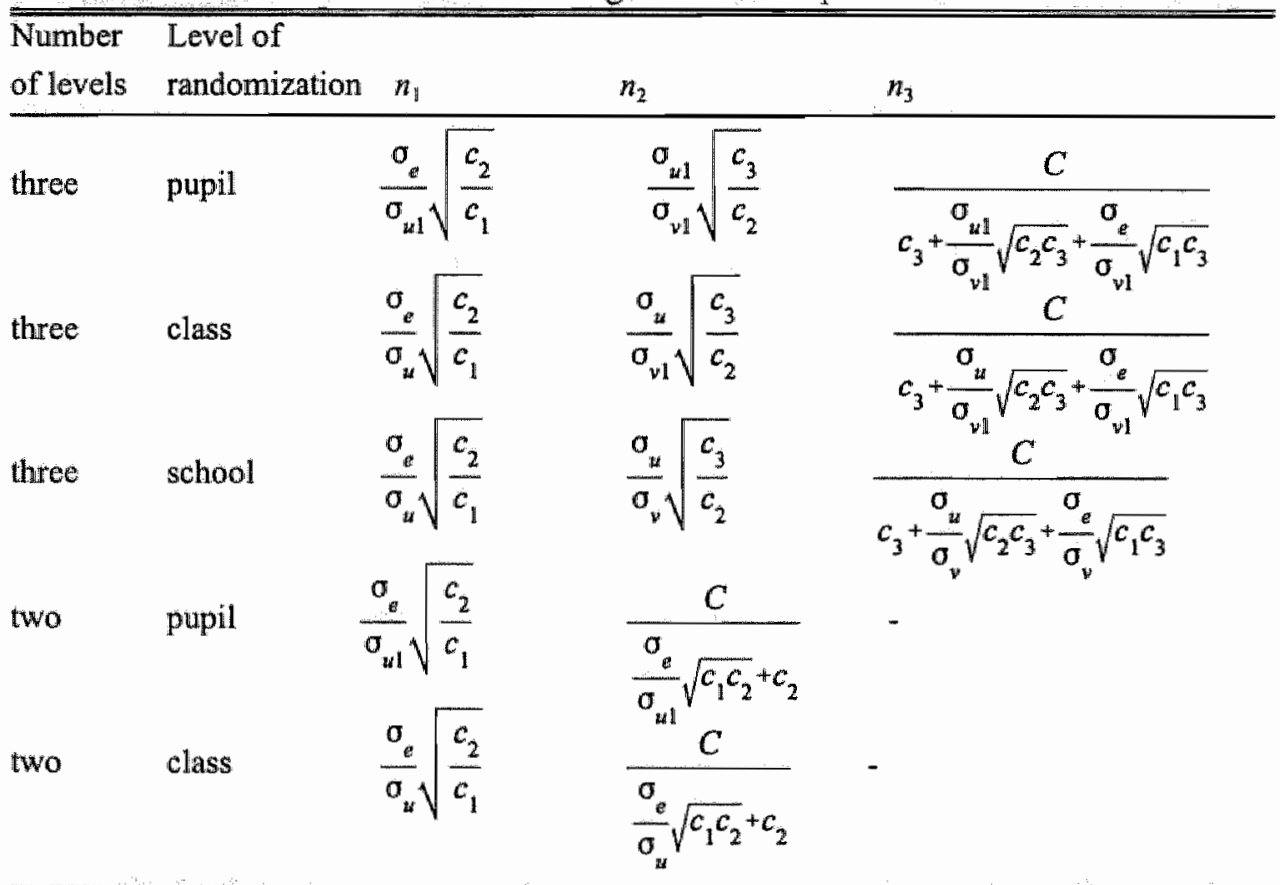

Note. For two level designs the nesting of classes within schools is omitted and thus the optimal $n_{3}$ is not given.

Treatment conditions are coded -1 and +1 . $\sigma_{u}^{2}=\sigma_{u 0}^{2}+\sigma_{w 1}^{2}, \quad \sigma_{v}^{2}=\sigma_{v 0}^{2}+\sigma_{v 1}^{2}$ 


\subsubsection{Optimal allocation of units}

Table 3.5 and 3.6 show the optimal allocation of units and the $\operatorname{Var}\left(\beta_{1}\right)$ given the optimal allocation of units. It follows from these tables that the optimal number of classes and schools for randomization at the pupil level is larger than for models without random slopes. Furthermore, a larger budget for sampling and measuring $C$ will lead to sampling more units at the highest level of the multilevel data structure, regardless of the level of randomization.

Note that Table 3.5 does not reduce to Table 3.2 when $\sigma_{u 1}^{2}$ and $\sigma_{v 1}^{2}$ go to zero, except when randomization is done at the highest level. This is a consequence of the fact that the optimal sample sizes in Table 3.2 were calculated such that $n_{1}, n_{2}$, and $n_{3}$ are at least two, which is not necessarily the case for the optimal sample sizes in Table 3.5. A same reasoning holds for the reduction of Table 3.6 to Table 3.3 when $\sigma_{u 1}^{2}$ and $\sigma_{v 1}^{2}$ go to zero.

Table 3.6 $\operatorname{Var}\left(\hat{\beta}_{1}\right)$ under the Optimal Allocation of Units for each Level of Randomization and Assuming a Random Slope

\begin{tabular}{|c|c|c|}
\hline Number of levels & Level of randomization & $\operatorname{Var}\left(\hat{\beta}_{b}\right)$ \\
\hline three & pupil & $\frac{\left(\sigma_{v 1} \sqrt{c_{3}}+\sigma_{u 1} \sqrt{c_{2}}+\sigma_{e d} \sqrt{c_{11}}\right)^{2}}{C}$ \\
\hline three & class & $\frac{\left(\sigma_{11} \sqrt{c_{3}}+\sigma_{u} \sqrt{c_{2}}+\sigma_{e} \sqrt{c_{1}}\right)^{2}}{C}$ \\
\hline three & school & $\frac{\left(\sigma_{w} \sqrt{c_{3}}+\sigma_{u} \sqrt{c_{2}}+\sigma_{a} \sqrt{c_{1}}\right)^{2}}{C}$ \\
\hline two & pupil & $\frac{\left(\sigma_{u 1} \sqrt{c_{2}}+\sigma_{u} \sqrt{c_{1}}\right)^{2}}{C}$ \\
\hline two & class & $\frac{\left(\sigma_{u} \sqrt{c_{2}}+\sigma_{e} \sqrt{c_{1}}\right)^{2}}{C}$ \\
\hline
\end{tabular}

Note. Treatment conditions are coded -1 and +1 .

$\sigma_{u}^{2}=\sigma_{u 0}^{2}+\sigma_{u 1}^{2}, \quad \sigma_{v}^{2}=\sigma_{v 0}^{2}+\sigma_{v 1}^{2}$ 


\subsubsection{An example (continued)}

Suppose now that there are a random slope at both the pupil and class level. The values of the variance components are $\sigma_{u 0}^{2}=1.5, \sigma_{u 1}^{2}=0.5, \sigma_{v 0}^{2}=0.3, \sigma_{v 1}^{2}=0.2$, and $\sigma_{e}^{2}=16$. The costs and budget are equal to those in Section 3.3.3: $c_{1}=\$ 100, c_{2}=\$ 200, c_{3}=300$, and $C=\$ 20000$. Then the optimal allocation for randomization at the pupil level is equal to $n_{1}=8, n_{2}=1.94, n_{3}=8.94$ and $\operatorname{Var}\left(\hat{\beta}_{1}\right)=0.167$. For integer values the optimal samples sizes are $n_{1}=8, n_{2}=3, n_{3}=6$, the costs are equal to $\$ 19800$, and $\operatorname{Var}\left(\beta_{1}\right)=0.172$. For randomization at the cllass level a $\operatorname{Var}\left(\hat{\beta}_{1}\right)=0.229$ is achieved by the optimal allocation $n_{1}=4, n_{2}=3.87, n_{3}=7.62$. For integer values the optimal sample sizes are $n_{1}=4, n_{2}=6$, $n_{3}=5$, which gives costs equal to $\$ 19500$ and $\operatorname{Var}\left(\beta_{1}\right)=0.240$. Finally the optimal allocation of units for randomization at the school level is equal to $n_{1}=4, n_{2}=2.4$, and $n_{3}=11.3$ and $\operatorname{Var}\left(\beta_{1}\right)=0.261$. For integer values $n_{1}=6, n_{2}=2$, and $n_{3}=10$, the costs are equal to $\$ 19000$ and $\operatorname{Var}\left(\beta_{1}\right)=0.283$.

\subsection{Power of the test of no treatment effect}

Suppose we are testing the hypothesis that the population value for the regression coefficient $\beta_{1}$ associated with treatment effect is zero; that is $\mathrm{H}_{0}: \beta_{1}=0$. The third design issue is the budget required for this test to have a specified power when $\beta_{1}$ has a given value, say $\beta_{1}=\Delta$. The test statistic is $z=\hat{\beta}_{1} / \sqrt{\operatorname{Var}\left(\beta_{1}\right)}$; under $H_{0,} z$ has the standard normal distribution since the variance components are assumed known. For one-sided tests with alternatives $\mathrm{H}_{1}: \beta_{1}>0$ or $\mathrm{H}_{2}: \beta_{1}<0$, with power $1-\gamma$ at $\beta_{1}=\Delta$ and with significance level $\alpha$, we have (e.g., Cochran, 1983):

$$
\sqrt{\operatorname{Var}\left(\hat{\beta}_{1}\right)}=\frac{|\Delta|}{z_{1-\alpha}+z_{1-\gamma}}
$$

where $z_{1-\alpha}$ and $z_{1-\gamma}$ are the $100(1-\alpha) \%$ and $100(1-\gamma) \%$ standard normal deviates; $\Delta$ is of course positive for alternative $\mathrm{H}_{1}$ and negative for alternative $\mathrm{H}_{2}$. For two-sided tests with altemative $H_{3}: \beta_{1} \neq 0, z_{1-\alpha}$ in (3.11) is replaced by $z_{1-(\alpha-2) \text {. }}$.

In planning the experiment $\Delta$ is first chosen (bearing in mind that $\beta_{1}$ denotes half of the true between-group difference, because treatment conditions are coded -1 , and +1 ); the required value of $\operatorname{Var}\left(\hat{\beta}_{1}\right)$ is then given by $(3.11)$, and the required budget and allocation of units follow as in Section 3.3.4. This is illustrated in the following example.

\subsubsection{An example (continued)}

Suppose that three levels of nesting are distinguished and that we want to test $\mathrm{H}_{0}: \beta_{1}=0$ versus $\mathrm{H}_{\mathrm{a}}: \beta_{1}>0$ at a significance level $\alpha=0.05$ and with power $1-\gamma=0.90$ at the value $\Delta=1$ for $\beta_{1}$. From $(3.11), \operatorname{Var}\left(\beta_{1}\right)=[1 /(1.645+1.282)]^{2}=0.1167$. Suppose also that $c_{1}=\$ 100, c_{2}=\$ 200, c_{3}=\$ 300, \sigma_{e}^{2}=16, \sigma_{u 0}^{2}=2$, and $\sigma_{w 0}^{2}=0.5$, which is the set of 
parameter values we used in Section 3.3.3. Thus we have a model with fixed slopes. If randomization is done at the pupil level a budget of $C=\$ 15100$ is needed for this test. If randomization is done at the class level, we need a budget $C=\$ 31400$, and if randomization is done at the school level a budget of $C=\$ 44700$ is needed.

\subsection{Discussion and conclusions}

The first step in designing experiments in multilevel populations is the establishment of the optimal level of randomization. The lowest level is the optimal level of randomization. However, there may be practical reasons for randomization at a higher level, resulting in a higher required budget to obtain a certain power of the test of treatment effect. Once the required budget is calculated using (3.11), the optimal allocation follows from Table 3.2 .

In experiments the effect of the treatment condition is generally of main interest, although covariates may be included in the multilevel regression model. Therefore the derivation of the optimal level of randomization and the optimal allocation of units was based on the regression coefficient of this treatment factor. When the covariates, which may be fixed or random, are also of interest, a $D$-optimal design would probably be mone appropriate.

It must be emphasized that in this study a balanced design and randomization into equal numbers of individuals per treatment group were assumed, while it is also useful to derive optimal designs for more general cases. Furthermore the variance components were assumed known, while it is also necessary to establish the effect of unknown variance components on $\operatorname{Var}\left(\hat{\beta}_{1}\right)$. Research on this issue is now in progress. First results show that the formulas in Table 3.1 and 3.4 still hold for unknown variance components if these are replaced by their estimates. The optimal sample sizes were derived for estimating the treatment effect as efficiently as possible when the variance components are known, and it has to be noted that these sample sizes are not necessarily optimal for estimating the variance components. For example, the optimal number of classes is equal to two for two levels of nesting, randomization at the pupil level and a treatment effect that is constant across classes. With such small a number of classes the class level variance component $\sigma_{u 0}^{2}$ cannot be estimated very well, but the variance of the treatment effect estimator does not depend on this variance component and so the formulas for the optimal sample sizes as given in Table 3.2 may be expected to perform well if the treatment effect is the only parameter of interest. A similar reasoning holds for models with three levels of nesting and a fixed slope and randomization at the pupil or class level. One might also wonder if such a small number of classes will represent a sample from the population of classes so that the multilevel model may be used for the data analysis and the results may be generalized to the population of classes. A rule of thumb is given by Snijders and Bosker (1999, p. 44), but more research on this topic is necessary.

Furthermore, it is also necessary to establish the effect of unknown variance 
components on the distribution of the test statistic for the test of no treatment effect. For estimation by Restricted Iterative Generalized Least Squares (RIGLS, Goldstein, 1989) this test statistic is $t$-distributed if the variance components are unknown, and the degrees of freedom can be found in ANOVA tables since RIGLS estimates are equivalent to Restricted Maximum Likelihood Estimates under the normal case (see Goldstein, 1989), which in their turn are equivalent to ANOVA estimates for balanced designs, whether normality is assumed or not (see for more details Searle, Casella, and McCulloch, 1992, Sections 4.8 and 6.6f).

\section{References}

Brylk, A. S., \& Raudenbush, S. W. (1992). Hierarchical linear madels. Newbury Park: Sage Publications.

Cochran, W. G. (1983). Planning and analysis of abservational studies. New York: Wiley.

Donner, A., Birkett, N., \& Buck, C. (1981). Randomization by cluster, sample size requirements and analysis. American Journal of Epidemiology, 114, 906-914.

Donner, A., Brown, K. S., \& Brasher, P. (1990). A methodological review of non-therapeutic intervention trials employing cluster randomization, 1979-1989. International Journal of Epidemiology, 19, 795-800.

Gail, M. H., Mark, S. D., Carroll, R. J., Green, S. B., \& Pee, D. (1996). On design considerations and randomization-based inference for community intervention triais. Statistics in Medicine, 15, 1069-1092.

Goldstein, H. (1989). Restricted iterative generalized least-squares estimation. Biometrika, 76, 622623.

Goldstein, H. (1995). Multilewel Statistical Models, 2nd edn. London: Edward Amold.

Hsieh, F. Y. (1988). Sample size formulae for intervention studies with the cluster as unit of randomization. Statistics in medicine, 8, 1195-1201.

Long ford, N. T. (1995), Random coefficient models. Oxford: Clarendon Press.

Moerbeek, M.y Van Breukelen, G. J. P.,\& Berger, M. P. F. (1999). Optimal experimental designs for linear multilevel models with covariates. Manuscript submitted for publication.

Mok, M. (1996). Sample size requirements for 2-level designs in educational research. Multilevel Modelling Newslefter, 7, 11-16.

Raudenbush S. W. (1997). Statistical analysis and optimal design for cluster randomized trials. Psychological Methods, 2, 173-185.

Searle, S. R., Casella, G., \& McCulloch, C. E. (1992). Variance components. New York: John Wiley \&. Sons.

Snijders, T. A. B. \& Bosker, R. J. (1993). Standard enrors and sample sizes for two-level research. Journal of Educational Statistics, 18, 237-259.

Snijders, T. A. B., \& Bosker, R. J. (1999). Muttilevel analysis: An introduction to basic and advanced multilevel modelling. London: Sage Publications. 


\section{Appendix}

\section{Calculation of the $\operatorname{Var}\left(\beta_{1}\right)$ for known variance components}

In this appendix we will restrict to two levels of nesting (i.e. pupils within classes) and models with interaction between treatment condition and class. The $\operatorname{Var}\left(\hat{\beta}_{1}\right)$ for models without interaction is obtained by setting $\sigma_{u 1}^{2}=0$. The derivation of the $\operatorname{Var}\left(\hat{\beta}_{1}\right)$ for three levels of nesting is likewise and not given here. We make use of the fact that the multilevel model and the aggregated data model give the same results if the variance components are known. In the models below $x_{i j}$ is coded +1 for the intervention group and -1 for the control group.

The model with interaction between treatment condition and class is given by

$$
y_{i j}=\beta_{0}+\beta_{1} x_{i j}+u_{0 j}+u_{1 j} x_{i j}+e_{i j}
$$

For pupil level randomization the difference between the average outcomes in the two treatment groups in class $j$ is then equal to

$$
\left(\bar{y}_{j i}-\bar{y}_{j l}\right)=\left(\left(\beta_{0}+\beta_{1}+u_{0 j}+u_{1 j}+\bar{e}_{j i}\right)-\left(\beta_{0}-\beta_{1}+u_{0 j}-u_{1 j}+\bar{e}_{j e}\right)\right)=2 \beta_{1}+2 u_{i j}+\left(\bar{e}_{j t}-\bar{e}_{j k}\right),
$$

where the subscripts $t$ and $c$ denote the intervention and control group respectively. So $\beta_{1}$ may be estimated by

$$
\hat{\beta}_{1}=\frac{1}{n_{2}} \sum_{j=1}^{n_{2}} \frac{1}{2}\left(\bar{y}_{j t}-\bar{y}_{j l}\right)=\frac{1}{n_{2}} \sum_{j=1}^{n_{2}}\left(\beta_{1}+u_{1 j}+\frac{1}{2}\left(\bar{e}_{j t}-\bar{e}_{j c}\right)\right),
$$

which has variance

$$
\operatorname{Var}\left(\hat{\beta}_{1}\right)=\operatorname{Var}\left(\frac{1}{n_{2}} \sum_{j=1}^{n_{2}} \frac{1}{2}\left(\bar{y}_{j l}-\bar{y}_{j l}\right)\right)=\frac{1}{n_{2}^{2}} \sum_{j=1}^{n_{2}} \operatorname{Var}\left(u_{\mathrm{ij}}+\frac{1}{2}\left(\bar{e}_{j t}-\bar{e}_{j j}\right)\right)=\frac{n_{1} \sigma_{u 1}^{2}+\sigma_{\varepsilon}^{2}}{n_{1} n_{2}} .
$$

For class level randomization we have

$$
\begin{aligned}
& \bar{y}_{j}=\beta_{0}+\beta_{1}+u_{0 j}+u_{1 j}+\bar{e}_{j} \text { if } x_{j}=+1 \\
& \bar{y}_{j}=\beta_{0}-\beta_{1}+u_{0 j}-u_{1 j}+\bar{e}_{j} \text { if } x_{j}=-1
\end{aligned},
$$

and so $\beta_{1}$ may be estimated by 


$$
\beta_{1}=\frac{1}{2}\left(\frac{\sum_{j=1}^{\frac{1}{2} n_{2}} \bar{y}_{j}}{\frac{1}{2} n_{2}}-\frac{\sum_{j=\frac{1}{2} n_{2}+1}^{n_{2}} \bar{y}_{j j}}{\frac{1}{2} n_{2}}\right)=\beta_{1}+\frac{1}{2}\left(\frac{\sum_{j=1}^{\frac{1}{n_{2} n_{2}}} u_{0 j}+u_{j i j}+\bar{e}_{j j}}{\frac{1}{2} n_{2}}-\frac{\sum_{j=\frac{1}{2} n_{2}+1}^{n_{2}} u_{0 j}-u_{j j}+\bar{e}_{j}}{\frac{1}{2} n_{2}}\right),
$$

if the first $\frac{1}{2} n_{2}$ classes are in the treatment group and the others are in the control group. This statistic has variance

$$
\begin{aligned}
\operatorname{Var}\left(\beta_{1}\right) & =\frac{1}{4} \operatorname{Var}\left(\frac{\sum_{j=1}^{\frac{1}{2} n_{2}} \bar{y}_{j}}{\frac{1}{2} n_{2}}-\frac{\sum_{j=\frac{1}{2} n_{2}+1}^{n_{2}} \bar{y}_{j}}{\frac{1}{2} n_{2}}\right)=\frac{1}{4} \operatorname{Var}\left(\frac{\sum_{j=1}^{\frac{1}{2} n_{2}} u_{0 j}+u_{1 j}+\bar{e}_{j}}{\frac{1}{2} n_{2}}-\frac{\sum_{j=\frac{1}{2} n_{2}+1}^{n_{2}} u_{0 j}-u_{1 j}+\bar{e}_{j}}{\frac{1}{2} n_{2}}\right) \\
& =\frac{1}{4}\left(\frac{\sigma_{u 0}^{2}+\sigma_{u 1}^{2}+2 \sigma_{u 01}+\frac{\sigma_{e}^{2}}{n_{1}}}{\frac{1}{2} n_{2}}+\frac{\sigma_{u 0}^{2}+\sigma_{u 1}^{2}-2 \sigma_{u 01}+\frac{\sigma_{e}^{2}}{n_{1}}}{\frac{1}{2} n_{2}}\right)=\frac{n_{1}\left(\sigma_{u 0}^{2}+\sigma_{u 1}^{2}\right)+\sigma_{e}^{2}}{n_{1} n_{2}} .
\end{aligned}
$$




\title{
Chapter 4
}

\section{Optimal experimental designs for linear multilevel models with covariates}

\begin{abstract}
In this paper optimal experimental designs for multilevel models with covariates and two levels of nesting are considered. Multilevel models are used to describe the relationship between an outcome variable and a treatment condition and covariate. It is assumed that the outcome variable is measured on a continuous scale. As optimality criteria $c$ - optimality, $D$ - optimality, and $A$ - optimality are chosen. It is shown that pre-stratification on the covariate leads to a more efficient design and that the person level is the optimal level of randomization. Furthermore, optimal sample sizes are given and it is shown that these do not depend on the optimallity criterion when randomization is done at the group level. During the design stage the true model. and parameter values, including the variance components, are given and the effect of design choices on the optimality criteria is established, whereas during the analysis stage the true model and parameter values are unknown and the effect of model change on the estimated variance components is established. The implication of this issue is also considered in this paper.
\end{abstract}

Key words: Optimality Criteria, Level of Randomization, Sample Sizes, Pre-stratification 


\subsection{Introduction}

The aim of experiments is often to estimate the effect of a newly developed intervention or active treatment on the behavior or health of individuals in certain populations. An important issue in planning an experiment is the establishment of the sample size in both treatment groups. These sample sizes are often chosen in such a way that the variance of the treatment effect estimator is minimized, resulting in largest power of the test of no treatment effect. For the case of uncorrelated observations sample size formulas are widely known (e.g., Cochran, 1983).

In reality, however, observations are generally correlated such as in, for examplle, repeated measures designs with successive measures nested within individuals (for optimal designs see Tan and Berger, 1999; Hedeker, Gibbons, and Waternaux, 1999). Correlated observations also occur in multilevel populations where individuals are nested within groups. For instance, in school-based smoking prevention interventions pupils are nested within schools, and in multi-center clinical trials patients are nested within clinics. When designing experiments in multilevel populations not only the totall sample size has to be established, but also the sample sizes at each level of the multilevel data structure. For two levels of nesting the sample size formula for the case where randomization to treatment conditions is done at the group level are given by Donner (1998), Donner, Birkett, and Buck (1981), Feng and Grizzle (1992), Hsieh (1988), Lee and Dubin (1994), Liu and Liang (1997), and Shih (1997). Randomization may also be done at the individual level and the design issue of the optimal level of randomization is addressed by Moerbeek, Van Breukelen, and Berger (in press, 1999) for two and three levels of nesting.

These papers are restricted to models without covariates. In practice, however, covariates are often included in the data analysis for several reasons, notably to increase statistical power (in experiments), or to correct for confounding (in observational studies). Optimal sample sizes are given by Lipsitz and Parzen (1995) for uncorrelated observations, and by Raudenbush (1997) for group-level randomization. Snijders and Bosker (1993) give formulas for the optimal sample sizes in cases where all predictor variables are at the group level and show how numerical results can be obtained for models including predictor variables at the individual level. The present paper extends the results in these papers by also considering randomization at the individual level and the optimal level of randomization, and by using several optimality criteria.

In this paper optimal experimental designs for models with continuous outcomes, covariates, and two levels of nesting will be given. The optimal level of randomization and the optimal sample sizes will be established. Furthermore, it will be shown that a more efficient design can be obtained by eliminating the correlation between treatment condition and the covariate by using pre-stratification on the covariate. In the papers cited above the variance of the treatment effect estimator was used as optimality criterion while in the present paper $D$ - optimal and $A$ - optimal designs are considered as well. In the following section the multilevel model including the treatment condition and one covariate and corresponding estimators are given, using the notation of Goldstein (1995). Next the optimality criteria and the 
resulting optimal designs will be presented. Finally some attention is paid to the effect of including covariates on the estimated variance components in the actual data analysis. This is done for models with a fixed or a random treatment effect.

\subsection{Models and estimators for a fixed treatment effect}

For the sake of concreteness a multi-center clinical trial, with patients nested within clinics, is used as an example throughout this paper. It is assumed that the outcome variable is blood pressure, measured on a continuous scale, and that the design is balanced: there are $i=1, \ldots, n_{1}$ patients per clinic and $j=1, \ldots, n_{2}$ clinics. The treatment effect is considered fixed, a random treatment effect will be considered in Section 4.6. Randomization to the two treatment conditions (the active treatment and the placebo) may be done at the clinic level or at the patient level. If randomization is done at the patient level then within each clinic $\frac{1}{2} n_{1}$ patients are randomized to the active treatment group and the others are randomized to the placebo group, so $n_{1}$ has to be even. Thus, for randomization at the patient level treatment and clinic are crossed. Similarly, if randomization is done at the clinic level, $\frac{1}{2} n_{2}$ clinics are randomized to the active treatment group and the others to the placebo group and thus $n_{2}$ has to be even. In this case all patients within the same clinic receive the same treatment and thus clinics are nested within treatments.

The multilevel models which are introduced in this section describe the relationship between the outcome $y_{i j}$ on the one side and the treatment condition $x_{i j}$ and one covariate $c_{i j}$ (e.g., pre-treatment blood pressure) on the other side. It is assumed that $x_{i j}$ is coded -1 for the placebo group and +1 for the active treatment group and that $c_{i j}$ is grand mean centered, that is $\bar{c}_{. .}=0$. The covariate may vary at both the clinic and patient level (e.g., pre-treatment blood pressure), or at just one level (e.g., clinic type). When the covariate varies at both levels, it may be split into a component $\bar{c}_{j}$ that varies only between clinics and a component $c_{i j}-\bar{c}_{j}$ that varies only within clinics (e.g., Neuhaus and Kalbfleisch, 1998). The multilevel model for patient $i$ within clinic $j$ and with randomization at the patient levell is given by

$$
y_{i j}=\beta_{0}+\beta_{1} x_{i j}+\beta_{2} \bar{c}_{i j}+\beta_{3}\left(c_{i j}-\bar{c}\right)+u_{0 j}+e_{i j},
$$

where the random effects $u_{0 j}$ and $e_{l j}$ at the clinic and patient level have zero mean and variance $\sigma_{w 0}^{2}$ and $\sigma_{e}^{2}$, respectively, and are assumed to be independently distributed of each other and the predictor variables. Note that $x_{i j}$ may be replaced by $x_{j}$ for randomization at the clinic level.

When the covariate varies only at the clinic level the term $c_{i j}-\bar{c}_{j}$ is equal to zero and then $\beta_{3}\left(c_{i j}-\bar{c}_{j}\right)$ may be removed from equation (4.1). Similarly, when the covariate varies only at the patient level the term $\beta_{2} \bar{c}_{j}$ may be removed.

Model (4.1) can be expressed in matrix notation:

$$
y=X \beta+u+e
$$


where $y$ is a $n_{1} n_{2} \times 1$ wector of responses with its elements ordered by clinic, $X$ is a $n_{1} n_{2} \times p$ matrix of predictor variables, $\beta$ is a $p \times 1$ vector of regression coefficients and $u$ and $e$ are $n_{1} n_{2} \times \mathbb{1}$ wectors with random terms at the clinic and patient level, respectively. Note that $p=3$ for a covariate which varies at either the patient or the clinic level and that $p=4$ for a covariate which varies at both levels.

The covariance matrix $V$ of the responses is equal to

$$
V=\sigma_{e}^{2} I_{n_{1} n_{2}}+\sigma_{w 0}^{2} I_{n_{2}} \otimes J_{n_{1}}
$$

where $I$ is the identity matrix, $J$ is a square matrix having every element unity, and $\otimes$ is the Knonecker product. For known variance components $\sigma_{e}^{2}$ and $\sigma_{u 0}^{2}$ the Generalized Least Squares (GLS) estimator for $\beta$ is

$$
\beta=\left(X^{\prime} V^{-1} X\right)^{-1} X^{\prime} V^{-1} y,
$$

with covariance matrix of the estimators

$$
\operatorname{Var}(\beta)=\boldsymbol{M}^{-1}\left(\boldsymbol{X}, \sigma^{2}\right)=\left(\boldsymbol{X}^{\prime} \boldsymbol{V}^{-1} \boldsymbol{X}\right)^{-1},
$$

where $M\left(X, \sigma^{2}\right)$ is the $p \times p$ information matrix, and the vector $\sigma^{2}=\left(\sigma_{e}^{2}, \sigma_{t 00}^{2}\right)^{\prime}$ contains the variance components. For randomization at the clinic level

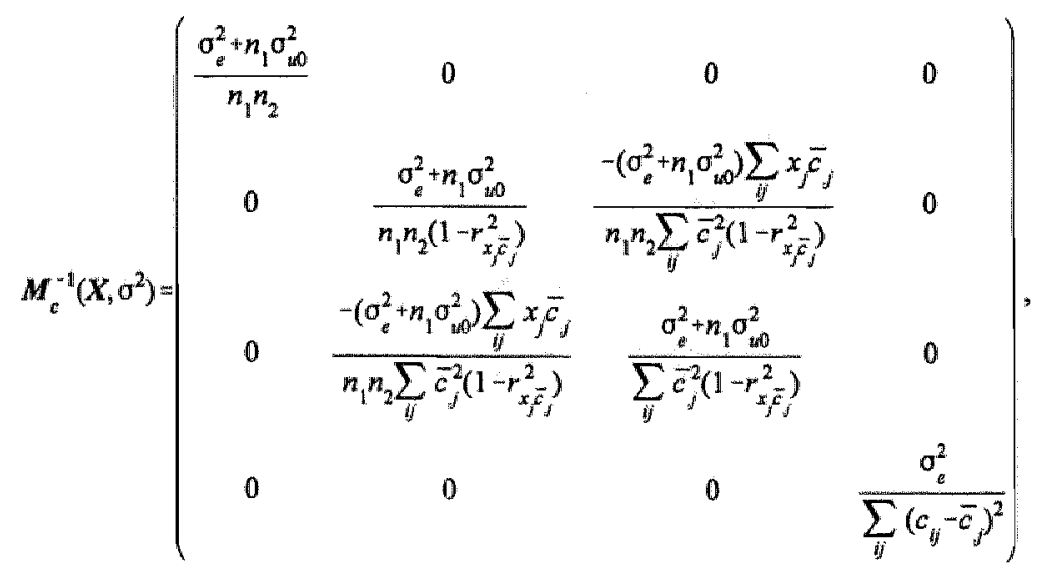


Whereas for randomization at the patient level

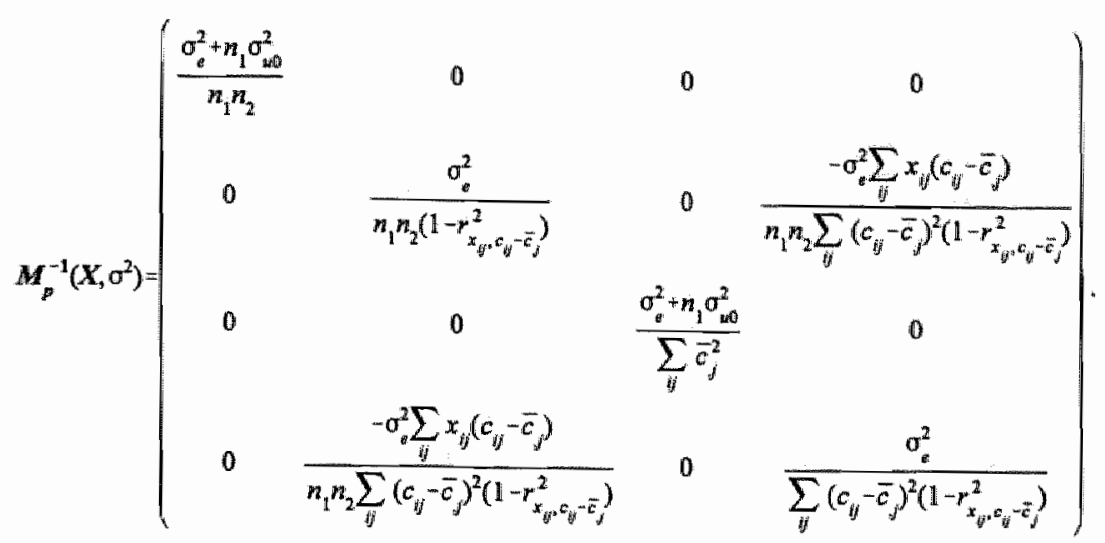

Note that in (4.6) and (4.7) the treatment condition is coded by -1 and +1 , implying that $\bar{x}=0$, and that the covariate is grand mean centered, i.e. $\vec{c} .=0$. The correlation between treatment condition and the covariate, henceforward shortly denoted by $r_{x c}^{2}$, is equal to $r_{x_{i}}^{2}$ for randomization at the clinic level and $r_{x_{i j} c_{i j}-\bar{c}_{j}}^{2}$ for randomization at the patient level. Note that for a covariate which varies only at the clinic level the fourth row and column may be erased from the covariance matrices. Similarly, the third row and column may be erased for a covariate which varies only at the patient level.

For unknown variance components iterative estimation procedures, such as Iterative Generalized Least Squares (IGLS, Goldstein, 1986) or Restricted Iterative Generalized Least Squares (RIGLS, Goldstein, 1989), have to be used. Then, (4.4) still holds and (4.5) asymptotically holds with $V$ replaced by its estimation.

\subsection{Design criteria}

In the above the notation $M\left(X, o^{2}\right)$ was used to stress that the information matrix depends on the design matrix $X$, and the vector of variance components $\sigma^{2}$. The elements of the design matrix $X$, in their turn, depend on the chosen design $\xi$ and the regression model $\eta$. Therefore, we will use the notation $\boldsymbol{M}\left(X(\xi, \eta), \sigma^{2}\right)$ rather than the short notation $\boldsymbol{M}(\xi)$, which is more common in optimal design literature, in the remainder of this chapter.

A design $\xi^{*}$ is said to be optimal if it is chosen such that a design criterion $\Psi\left(M\left(X(\xi, \eta), \sigma^{2}\right)\right)$ is minimized for all possible designs $\xi$ in the design space $\chi$. Note that a design criterion which does not depend on estimation bias was chosen, since GLS produces unbiased estimates. Various design criteria are available, of which three will be considered in this paper: $c$-optimality, $D$-optimality, and $A$ - optimality. With $c$-optimality a linear combination $c^{\prime} \beta$ of the regression coefficients is estimated with minimum variance: 


$$
\Psi\left(M\left(X(\xi, \eta), \sigma^{2}\right)\right)=c^{\prime} M^{-1}\left(X(\xi, \eta), \sigma^{2}\right) c .
$$

In the papers cited in the introduction, for example, the treatment effect was estimated with minimum variance. Thus $c=(0,1,0,0)^{\prime}$ where the last entry of $c$ may be erased if the covariate varies only at one level. This kind of $c$-optimality will also be used in this paper.

Two other frequently used design criteria are $D$ - optimality and $A$ - optimality in which the determinant and the trace of the inverse information matrix are minimized, respectively. As (4.6) and (4.7) show, the inverse information matrix depends on the variance of the covariate which is often an observational variable and not under experimental control. So we will restrict ourselves to the intercept $\beta_{0}$ and treatment effect $\beta_{1}$. The $D$-optimality criterion then reduces to a $D_{A}$ - optimality criterion (Atkinson and Donev, 1996, section 10.2) where

$$
\Psi\left(\boldsymbol{M}\left(\boldsymbol{X}(\xi, \eta), \sigma^{2}\right)\right)=\operatorname{det}\left(\boldsymbol{A}^{\prime} \boldsymbol{M}^{-1}\left(\boldsymbol{X}(\xi, \eta), \sigma^{2}\right) \boldsymbol{A}\right) \text {, where } \boldsymbol{A}^{\prime}=\left(\begin{array}{llll}
1 & 0 & 0 & 0 \\
0 & 1 & 0 & 0
\end{array}\right),
$$

and the $A$ - optimality criterion reduces to an $A_{A}$ - optimality criterion (which is referred to as $L$ - optimality criterion by Atkinson and Donev, 1996, section 10.6) where

$$
\Psi\left(\boldsymbol{M}\left(\boldsymbol{X}(\xi, \eta), \sigma^{2}\right)\right)=\operatorname{trace}\left(A^{\prime} \boldsymbol{M}^{-1}\left(\boldsymbol{X}(\xi, \eta), \sigma^{2}\right) A\right), \text { where } A^{\prime}=\left(\begin{array}{llll}
1 & 0 & 0 & 0 \\
0 & 1 & 0 & 0
\end{array}\right) .
$$

Note that the last row of the matrix $A$ may be erased if the covariate varies only at one level.

Another approach is to divide the regression coefficients into two subsets such that the first subset contains the $s$ regression coefficients of interest. The information matrix $M\left(X(\xi, \eta), \sigma^{2}\right)$ is then partitioned into

$$
\left(\begin{array}{ll}
M_{11}\left(X(\xi, \eta), \sigma^{2}\right) & M_{12}\left(X(\xi, \eta), \sigma^{2}\right) \\
M_{21}\left(X(\xi, \eta), \sigma^{2}\right) & M_{22}\left(X(\xi, \eta), \sigma^{2}\right)
\end{array}\right),
$$

where $M_{11}\left(X(\xi, \eta), \sigma^{2}\right)$ is the $s \times s$ submatrix corresponding to the regression coefficients of interest, and $M_{22}\left(X(\xi, \eta), \sigma^{2}\right)$ is the $(p-s) \times(p-s)$ submatrix corresponding to the remaining regression coefficients. The $D$ - optimality criterion then extends to a $D_{S}$ - optimality criterion (Atkinson and Donev, 1996, section 10.3) with

$$
\Psi\left(\boldsymbol{M}\left(\boldsymbol{X}(\xi, \eta), \sigma^{2}\right)\right)=\frac{\operatorname{det}\left(\boldsymbol{M}_{22}\left(\boldsymbol{X}(\xi, \eta), \sigma^{2}\right)\right)}{\operatorname{det}\left(\boldsymbol{M}\left(\boldsymbol{X}(\xi, \eta), \sigma^{2}\right)\right)} .
$$

This criterion is especially relevant when $M_{22}\left(X(\xi, \eta), \sigma^{2}\right)$ corresponds to the covariates which are not under experimental control. In this paper a design where $\beta_{1}$ is estimated as precisely as possible will be referred to as a $D_{S !}$ - optimal design, and a design where $\beta_{0}$ and $\beta_{1}$ are estimated as precisely as possible will be referred to as a $D_{S 2}$ - optimal design. 
Table 4.1. Design Criteria for Both Levels of Randomization and Models with a Fixed Treatment Effect

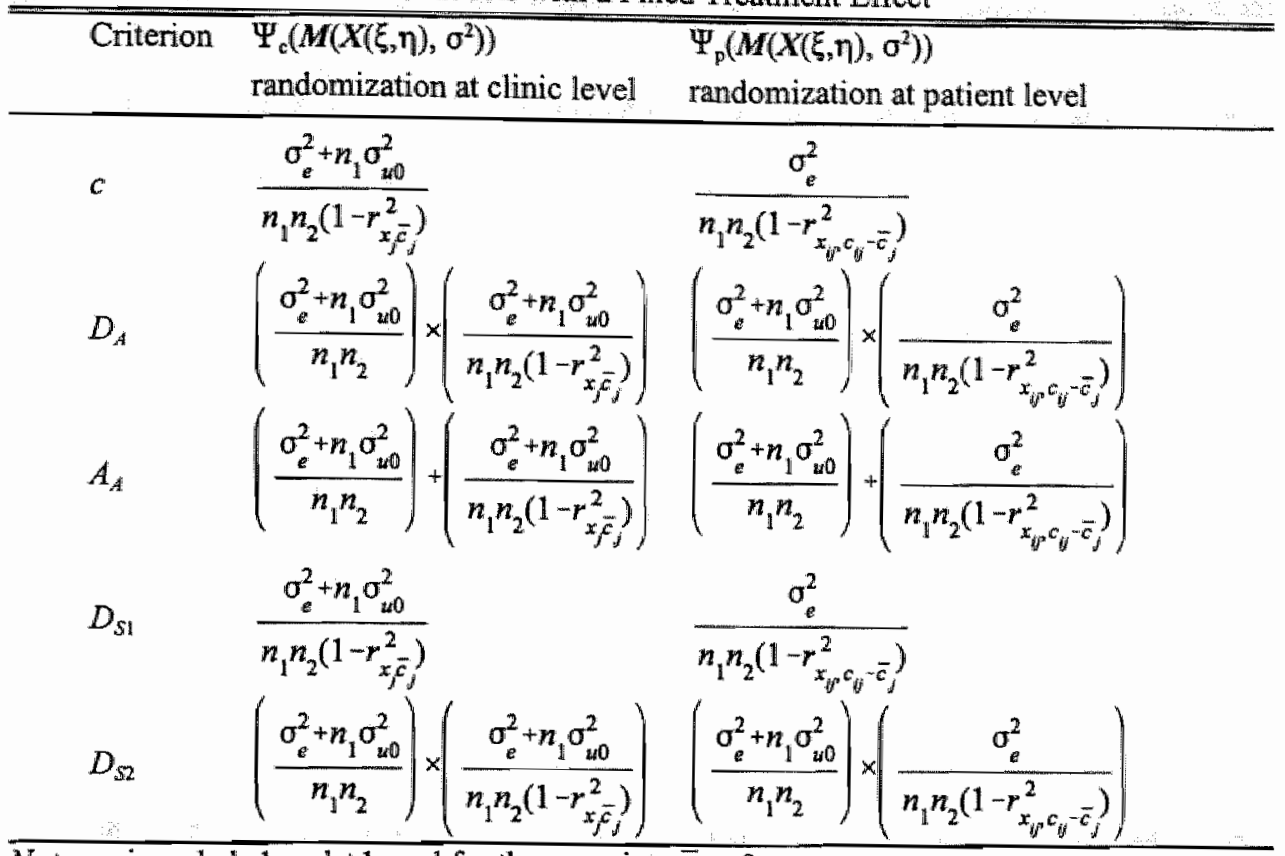

Note. $x_{i j}$ is coded -1 and +1 , and for the covariate $\bar{c}=0$.

\subsection{Optimal designs for models with a fixed treatment effect}

During the design stage it is assumed that model (4.1) is the true underlying model for the outcome variables and that the true regression coefficients $\beta_{0}, \beta_{1}, \beta_{2}$, and $\beta_{3}$, and true variance components $\sigma^{2}$ and $\sigma_{w 0}^{2}$ do not depend on the level of randomization or on the correlation between treatment condition and the covariate. Table 4.1 shows how the design criteria $\Psi\left(M\left(X(\xi, \eta), \sigma^{2}\right)\right)$ depend on the level of randomization, the variance components, the sample sizes at both levels, and the correlation between treatment condition and the covariate. As follows from Table 4.1 the $c-, D_{A}-, A_{A}-, D_{S 1}-$, or $D_{S 2}$ - efficiency of a diesign increases when the correlation $r_{x c}$ between treatment condition and outcome goes to zero, which can be approached by using large sample sizes. For normally and binary distributed covariates $r_{x c}$ is approximately normally distributed with variance $1 / n$ (Kendall and Stuart, 1979) and thus $r_{x c}{ }^{2} \in(0,4 / m)$ with $95 \%$ probability. Here, $n$ is equal to $n_{1} n_{2}$ or $n_{2}$ for randomization at the patient or clinic level, respectively, showing that the correlation $r_{\mathrm{xc}}$ will be small for large sample sizes, especially for randomization at the patient level. When large sample sizes are not achievable, a correlation $r_{x c}$ equal to zero can be achieved by pre-stratification on the covariate. When randonization to treatment conditions is done at the clinic level, $x_{j}$ and $c_{l j}-\bar{c}_{j}$ are automatically orthogonal, so prestratification has to be done on $\bar{c}_{j}$, which means that for each value of $\bar{c}_{j}$, half of the clinics are 
randomized to the intervention group whille the others are randomized to the control group. Similarly, when randomization to treatment conditions is done at the patient level, $x_{i j}$ and $\bar{c}_{j}$ are automatically orthogonal, so pre-stratification has to be done on $c_{i j}-\bar{c}_{j,}$. Note that a correlation equal to zero can be achieved exactly by pre-stratification when the covariate is binary, whereas for continuous covariates this is only approximately true. In two specific cases treatment condition and the covariate are always orthogonal and thus pre-stratification is not necessary: when randomization to treatment conditions is done at the clinic level and the covariate varies only at the patient level (i.e $\bar{c}_{j}=0, \forall j$ ), and when randomization to treatment conditions is done at the patient level and the covariate varies only at the clinic level (i.e. $c_{i j}-\bar{c}_{j}=0, \forall_{i j}$ ).

Furthermore it should be noted that $\Psi\left(\boldsymbol{M}\left(\boldsymbol{X}(\xi, \eta), \sigma^{2}\right)\right)$ for $D_{S 1}$ - optimality and for $D_{S 2}$ optimality is equal to $\Psi\left(M\left(X(\xi, \eta), \sigma^{2}\right)\right)$ for $c$ - optimality and for $D_{A}$ - optimality, respectively. For the $D_{A}$ - and the $D_{S z}$ - optimality criterion the variances of the random intercept and fixed slope are multiplied, whereas these variances are added for the $A_{A}$ - optimality criterion.

Table 4.1 also shows that the patient level is the optimal level of randomization given a certain value of $r_{x c}$, in particular given $r_{x c}=0$ (e.g., due to pre-stratification). In the remainder of this paper it is assumed that the correlation between treatment condition and the covariate is equal to zero, due to pre-stratification or large sample sizes.

In some cases randomization at the patient level is not possible and randomization has to be done at the clinic level. Reasons for randomization at the clinic level are political and administrative factors, ethical concerns, the need to reduce costs and treatment group contamination, which occurs when information may leak from the intervention group to the control group. In some cases the intervention cannot be implemented at the individual level, for example in class-based smoking prevention programs. Therefore, optimal sample sizes will be derived for both levels of randomization. To obtain the optimal sample sizes $\Psi\left(M\left(X(\xi, \eta), \sigma^{2}\right)\right)$ is minimized subject to the condition that the costs for measuring and sampling units may not exceed some fixed total budget $C$ for measuring and sampling units:

$$
c_{1} n_{1} n_{2}+c_{2} n_{2}=C \quad\left(c>0, n_{l} \geq 2 \text { for } l=1,2\right),
$$

and assuming that $r_{x c}=0$. Here $c_{2}$ and $c_{1}$ are the costs for measuring and sampling a clinic and a patient in an already sampled clinic, respectively. The optimal samples sizes can be derived by expressing $n_{2}$ in terms of $n_{1}, c_{1}, c_{2}$, and $C$ using (4.13), substituting this expression in $\Psi\left(M\left(X(\xi, \eta), \sigma^{2}\right)\right)$ as given in Table 4.1, and solving for $n_{1}$.

For both levels of randomization the optimal sample sizes are given in Table 4.2 and the value of the design criterion given the optimal sample sizes, $\Psi\left(\boldsymbol{M}\left(\left(\boldsymbol{X}\left(\xi^{*}, \eta\right), \sigma^{2}\right)\right)\right.$, is given in Table 4.3. From Table 4.2 it is clear that the optimal sample sizes do not depend on the design criterion if randomization is done at the clinic level. Furthermore, Table 4.2 shows that when $c$ - optimality is applied, increasing the budget $C$ leads to sampling more units only at the level at which randomization is done. For this optimality criterion it also holds that the optimal sample sizes do not depend on the variance components when randomization is done at the patient level. 
In that case the optimal number of clinics is equal to two. With such a small number of clinics the variance component at the clinic level can not be estimated very well, bat this parameter is not involved in the variance of the treatment effect estimator, see Table 4.1 and 4.3 . For randomization at the clinic level, and for models with a random treatment effect the wariance of the treatment effect estimator is a function of the variance component at the clinic level. But for these cases the optimal number of clinics is generally larger than two, and the variance component at the clinic level may be estimated better. Another objection is that such a small number of clinics cannot be considered a random sample from their population so that the multilevel model may not be used for the data analysis and the fixed effects model has to be used coding clinics with dummy variables. A rule of thumb for choosing between the multilevel model and the fixed effects model is given by Snijders and Bosker (1999, p. 44), but more research on this issue is necessary. If prior knowledge of the values of the variance components is not available but a preliminary guess of the size of the intra-clinic correlation coefficient $\rho_{c}=\sigma_{u 0}^{2} /\left(\sigma_{e}^{2}+\sigma_{u 0}^{2}\right)$ can be given, $\sigma_{e}^{2}$ and $\sigma_{u 0}^{2}$ may be replaced by $\left(1-\rho_{k}\right)$ and $\rho_{c}$, respectively, in the formulas for the optimal sample sizes in Table 4.2. Furthermore, it follows from Table 4.3 that a larger budget $C$ results in a more efficient design.

Table 4.2. Optimal Sample Sizes for Both Levels of Randomization and Models with a Fixed Treatment Effect

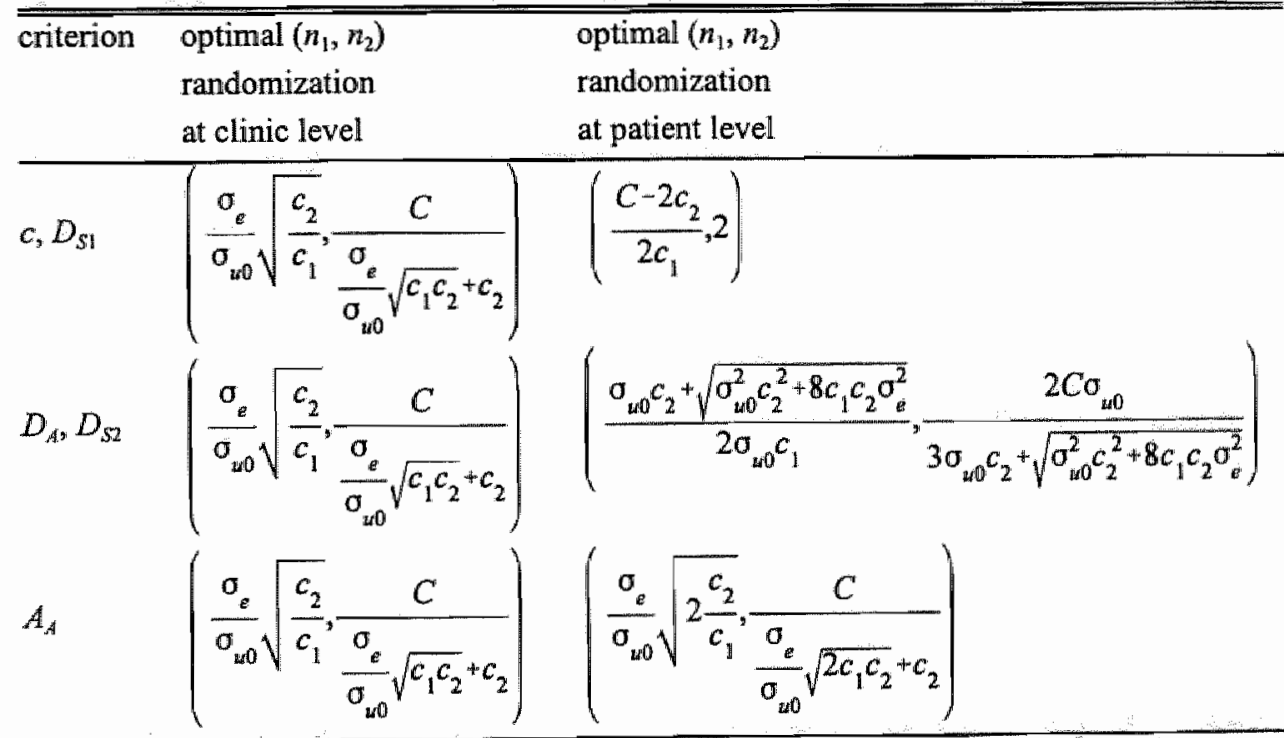

Note. $x_{i j}$ is coded -1 and +1 , and for the covariate $\bar{c}_{.}=0$. It is assumed that $r_{x c}{ }^{2}=0$ due to prestratification on the covariate or large sample sizes. 
Table 4.3. Optimal Values of the Design Criteria for Both Levels of Randomization and Models with a Fixed Treatment Effect

criterion $\Psi_{\mathrm{c}}\left(M\left(X\left(\xi^{*} ; \eta\right), \sigma^{2}\right)\right) \quad \Psi_{\mathrm{p}}\left(M\left(X\left(\xi^{*}, \eta\right), \sigma^{2}\right)\right)$

randomization at clinic level randomization at patient level

$c, D_{s i} \quad \frac{\left(\sigma_{e} \sqrt{c_{1}}+\sigma_{\mu \sigma 0} \sqrt{c_{2}}\right)^{2}}{C} \quad \frac{\sigma_{e}^{2} c_{1}}{C-2 c_{2}}$

$D_{A}, D_{S 2} \quad\left(\frac{\left(\sigma_{\left.s \sqrt{c_{1}}+\sigma_{k 0} \sqrt{c_{2}}\right)^{2}}\right.}{C}\right)^{2}$

$\sigma_{e}^{2}\left(\sigma_{e}^{2}+\frac{c_{2} \sigma_{u 0}+\sqrt{c_{2}^{2} \sigma_{u 0}^{2}+8 c_{1} c_{2} \sigma_{e}^{2}}}{2 \sigma_{u 0} c_{1}} \sigma_{u 0}^{2}\right)^{\times}$
$\left(\frac{c_{1}\left(3 c_{2} \sigma_{u 0}+\sqrt{c_{2}^{2} \sigma_{u 0}^{2}+8 c_{1} c_{2} \sigma_{e}^{2}}\right)}{C\left(c_{2} \sigma_{u 0}+\sqrt{c_{2}^{2} \sigma_{u 0}^{2}+8 c_{1} c_{2} \sigma_{e}^{2}}\right.}\right)^{2}$

$A_{A} \quad \frac{\left(\sigma_{e} \sqrt{2 c_{1}}+\sigma_{u 0} \sqrt{2 c_{2}}\right)^{2}}{C}$

$\frac{\left(\sigma_{e} \sqrt{2 c_{1}}+\sigma_{u v \sqrt{c_{2}}}\right)^{2}}{C}$

Note. $x_{i j}$ is coded -1 and +1 , and for the covariate $\bar{c}_{. s}=0$. It is assumed that $r_{x c}{ }^{2}=0$ due to prestratification on the covariate or large sample sizes.

For the $A_{A}$-, and $c$ - optimal design criterion the relative efficiency of randomization at the clinic level versus randomization at the patient level is given by

$$
\frac{\Psi_{c}\left(M\left(X\left(\xi^{*}, \eta\right), \sigma^{2}\right)\right)^{-1}}{\Psi_{p}\left(M\left(X\left(\xi^{*}, \eta\right), \sigma^{2}\right)\right)^{-1}}
$$

with $\Psi_{v}\left(\boldsymbol{M}\left(\boldsymbol{X}\left(\xi^{*}, \eta\right), \sigma^{2}\right)\right)$ and $\Psi_{\mathrm{p}}\left(\boldsymbol{M}\left(\boldsymbol{X}\left(\xi^{*}, \eta\right), \sigma^{2}\right)\right)$ as in Table 4.3 (i.e. assuming optimal sample sizes $n_{1}$ and $\left.n_{2}\right)$, and this ratio is always between zero and one. Its inverse gives the number of times the optimal design for randomization at the clinic level needs to be replicated to be as efficient as the optimal design for randomization at the patient level. To maintain this nice interpretation, the square root of the right-hand side of (4.14) has to be taken for $D_{A}$ - optimality. The relative efficiency for the $c$ - optimality criterion is given in Figure 4.1 as a function of the intra-clinic correlation coefficient $\rho_{c^{\prime}}$. This efficiency is given for several values of the cost ratio $c_{2} / c_{1}$, and for $C / c_{1}=5000$. For the $D_{A}$ - optimal design criterion and the $A_{A}$ - optimal design criterion the relative efficiency does not depend on $C / c_{1}$ and is given in Figures 4.2 and 4.3, respectively. From Figures $4.1,4.2$, and 4.3 it follows that randomization at the patient level is especially preferable for large intra-clinic correlation coefficients and large cost ratios $c_{2} / c_{11}$. 


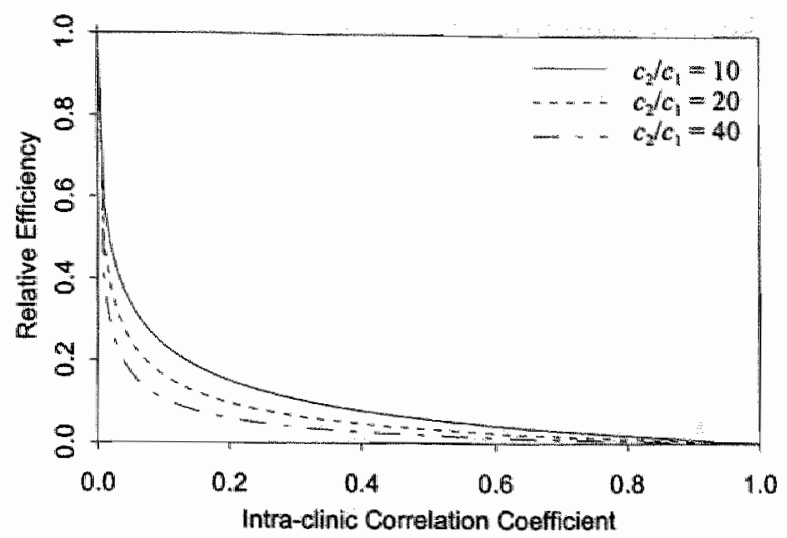

Figure 4.1. Relative efficiency of clinic versus patient randomization for $c$-optimal designs as a function of the intra-clinic conrelation coefficient and the cost ratio $c_{2} / c_{1}$, and for $C / c_{1}=5000$.

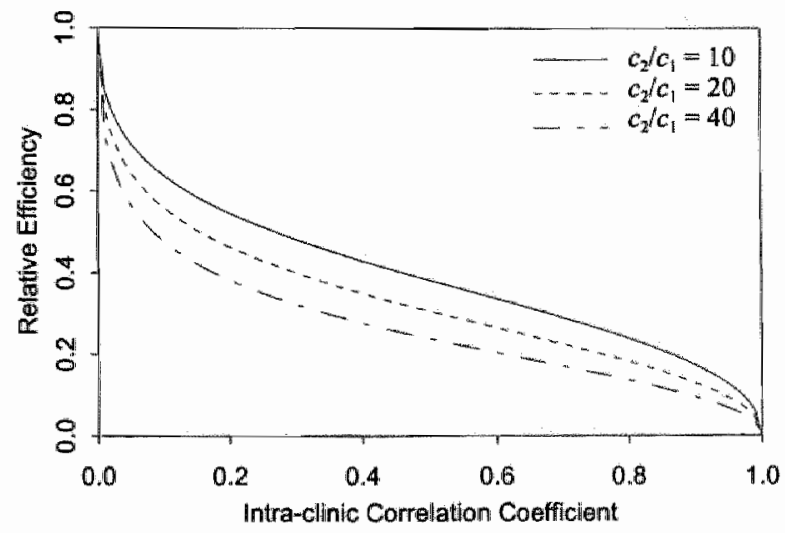

Figure 4.2. Relative efficiency of clinic versus patient randomization for $D_{A}$ - optimal designs as a function of the intra-clinic correlation coefficient and the cost ratio $c_{2} / c_{1}$. 


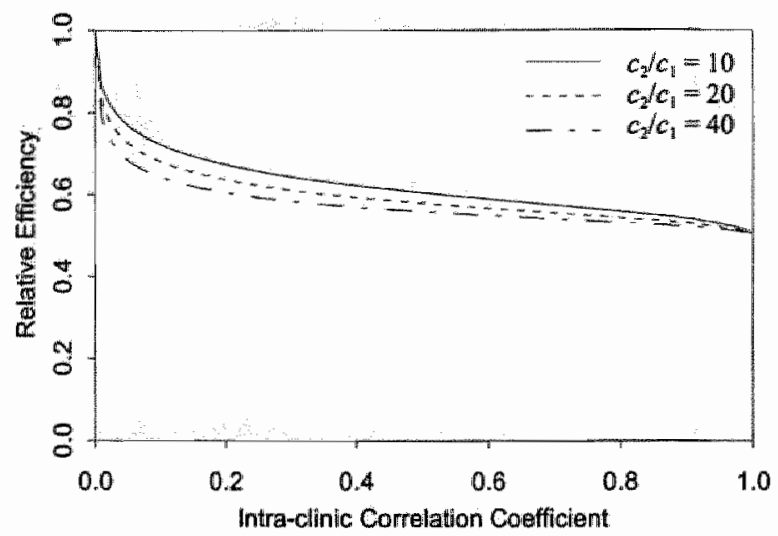

Figure 4.3. Relative efficiency of clinic versus patient randomization for $A_{A}$ - optimal designs as a function of the intra-clinic correlation coefficient and the cost ratio $c_{2} / c_{1}$.

\subsection{Effects on the estimated variance components of excluding covariates} from the model with a fixed treatment effect

Including covariates affects the optimal design and precision of the parameter estimates, not only through the possible correlation between treatment condition and the covariates, but also through the effect of including covariates on the variance components.

In the design stage it was assumed that model (4.1) is the true underlying model and that parameter values, including the variance components, are independent of the level of randomization and of possible pre-stratification on the covariate. Design choices affect the total observed outcome variance. During the analysis stage the reverse occurs: the total variation in the outcome $y_{i j}$ is given by the observed data and the estimated variance components change if covariates are added to or excluded from the model, i.e. if we no longer assume that the true model is known.

Suppose that both components $\bar{c}_{j}$ and $c_{i j}-\bar{c}_{j}$ of the covariate are excluded from the model (4.1), so that we obtain

$$
y_{i j}=\beta_{0}^{*}+\beta_{i}^{*} x_{i j}+u_{i j}^{*}+e_{i j}^{*}
$$

where the random effects $u_{j}^{*}$ and $e_{i j}{ }^{*}$ have variance $\sigma_{u 0^{*}}^{2}$ and $\sigma_{e}^{2}$, respectively. The change in the estimated variance components can be derived by assuming that the variance of the observed outcomes, $\operatorname{var}\left(y_{i j}\right)$, and the covariance of two outcomes within the same clinic, $\operatorname{cov}\left(\gamma_{i j} ; y_{i j}\right)$ are given by the data, and therefore equal for model (4.1) and model (4.15), i.e. 


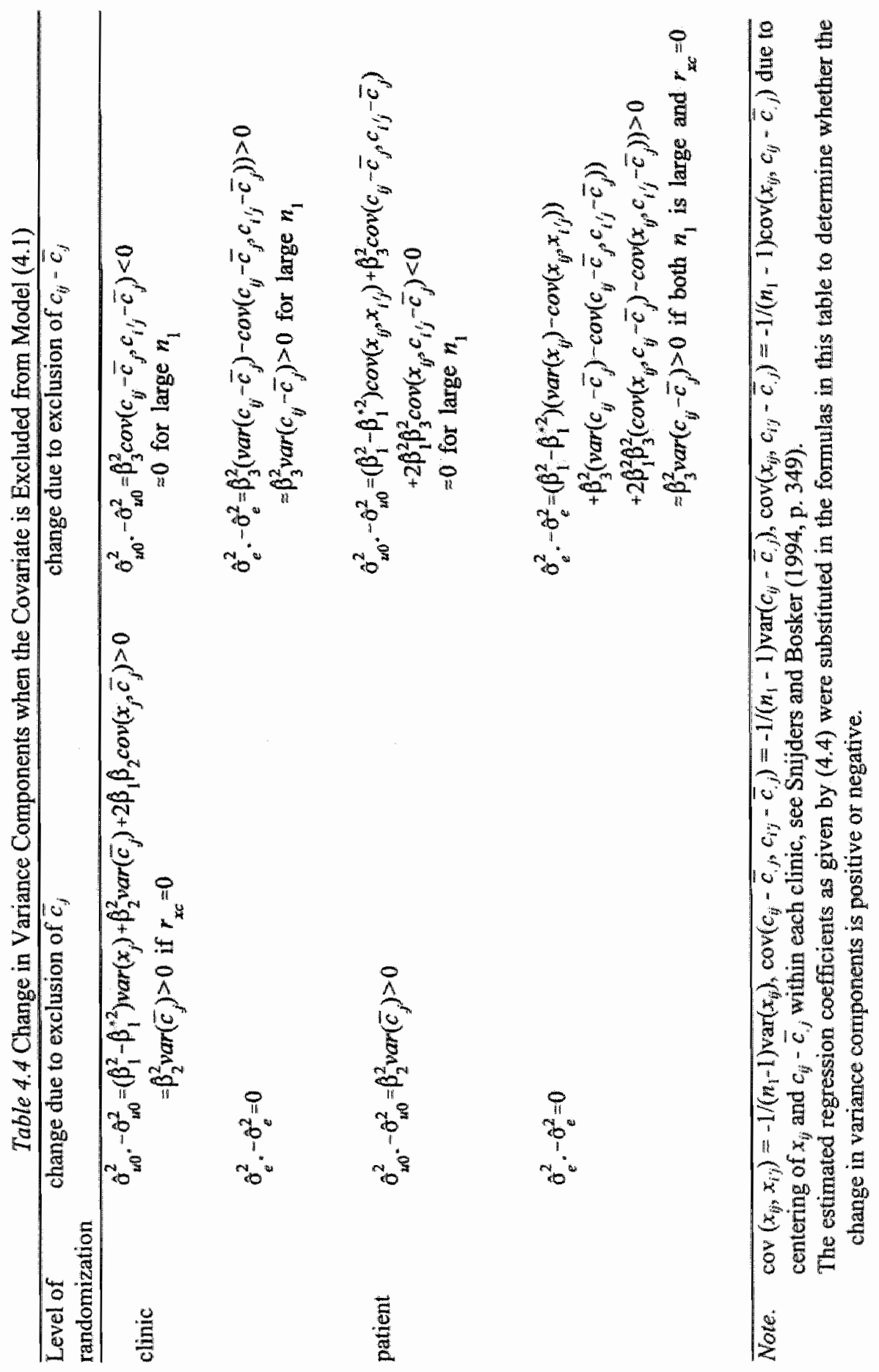




$$
\begin{aligned}
\operatorname{Var}\left(y_{i j}\right) & =\operatorname{Var}\left(\hat{\beta}_{1}^{*} x_{i j}+\hat{u}_{i j}^{*}+\hat{e}_{i j}^{*}\right) \\
& =\operatorname{Var}\left(\hat{\beta}_{1} x_{i j}+\hat{\beta}_{2} \bar{c}_{j}+\hat{\beta}_{3}\left(c_{i j}-\bar{c}_{j}\right)+\hat{u}_{0 j}+\hat{e}_{i j}\right),
\end{aligned}
$$

and

$$
\begin{aligned}
& \operatorname{Cov}\left(y_{i p^{\prime}} y_{i^{\prime}}\right)=\operatorname{Cov}\left(\beta_{1}^{*} x_{i j}+\hat{u}_{0 j^{\prime}}^{*} \beta_{1}^{*} x_{i^{\prime} j}+\hat{u}_{0, j^{*}}^{*}\right) \\
& =\operatorname{Cov}\left(\hat{\beta}_{1} x_{i j}+\hat{\beta}_{2} \bar{c}_{j}+\hat{\beta}_{3}\left(c_{i j}-\bar{c}_{j j}\right)+\hat{u}_{0 j^{\prime}} \hat{\beta}_{1} x_{i j}+\hat{\beta}_{2} \bar{c}_{j j}+\hat{\beta}_{3}\left(c_{f^{\prime} j}-\bar{c}_{j j}\right)+\hat{u}_{0 j}\right),
\end{aligned}
$$

where $x_{i j}$ may be replaced by $x_{j}$ if randomization is done at the clinic level. This method to establish the change in estimated variance components was also used by Snijders and Bosker (1994). For both levels of randomization the change in the estimated variance components due to exclusion of the separate components $\bar{c}_{j}$ or $c_{i j}-\bar{c}_{j}$ of the covariate is given in Table 4.4. The total change due to exclusion of both components $\bar{c}_{j}$ and $c_{i j}-\bar{c}_{j}$ of the covariate is equal to the sum of the change due to exclusion of the separate components.

From Table 4.4 it follows that the estimated variance component at the clinic level increases whereas the estimated variance component at the patient level remains constant when a covariate which varies at the clinic level is excluded from model (4.1). Consequently the $\operatorname{Var}\left(\beta_{1}\right)$ will increase if randomization is done at the clinic level and $r_{x c}=0$, and remain unchanged when randomization is done at the patient level. The estimated variance component at the patient level increases if a covariate which varies at the patient level is excluded from the model. The estimated variance component at the clinic level then decreases slightly due to the fact that $x_{i j}$ and $c_{i j}-\bar{c}_{j j}$ are group mean centered. The $\operatorname{Var}\left(\hat{\beta}_{1}\right)$ will increase if randomization is done at the patient level and $r_{x c}=0$, and remain unchanged for randomization at the clinic level since the increase in the patient level variance component is $n_{1}$ times as large as the decrease in the clinic level variance component.

\subsection{Optimal designs for models with a random treatment effect}

When clinic by treatment interaction is expected to be present a random slope has to be added to the multilevel model in (4.1) and we then get

$$
y_{i j}=\beta_{0}+\beta_{1} x_{i j}+\beta_{2} \bar{c}_{j}+\beta_{3}\left(c_{i j}-\bar{c}_{j}\right)+u_{0 j}+u_{i j} x_{i j}+e_{i j}
$$

where $u_{1 j} \sim \mathrm{N}\left(0, \sigma_{u 1}^{2}\right)$ is the clinic effect associated with the random treatment effect. This term may correlate with the random term $u_{0 j}$ and their covariance is denoted $\sigma_{u: 01}$. When randomization is done at the clinic level there is only one treatment condition per clinic, and consequently $\sigma_{u 0}^{2}$ and $\sigma_{u !}^{2}$ cannot be estimated separately. Instead their sum is estimated which will be denoted $\sigma_{u}^{2}$.

We will only present optimal designs for the case where treatment condition is 
uncorrelated with both components $c_{i j}-\bar{c}_{j}$ and $\bar{c}_{j}$ of the covariate. In that specific case only the variances and covariance of the estimated intercept and estimated treatment effect are needed to calculate the $C$-, the $D_{A}-$, and the $A_{A}$ - optimal designs, and are given by

$$
A^{\prime} M_{p}^{-1}\left(X(\xi, \eta), \sigma^{2}\right) A=\left(\begin{array}{cc}
\operatorname{Var}\left(\hat{\beta}_{0}\right) & \operatorname{Cov}\left(\hat{\beta}_{0^{0}}, \hat{\beta}_{1}\right) \\
\operatorname{Cov}\left(\hat{\beta}_{0}, \hat{\beta}_{1}\right) & \operatorname{Var}\left(\hat{\beta}_{1}\right)
\end{array}\right)=\left(\begin{array}{cc}
\frac{\sigma_{e}^{2}+n_{1} \sigma_{u 0}^{2}}{n_{1} n_{2}} & \frac{n_{1} \sigma_{u 01}}{n_{1} n_{2}} \\
\frac{n_{1} \sigma_{u 01}}{n_{1} n_{2}} & \frac{\sigma_{e}^{2}+n_{1} \sigma_{u 1}^{2}}{n_{1} n_{2}}
\end{array}\right),
$$

for randomization at the patient level, and by

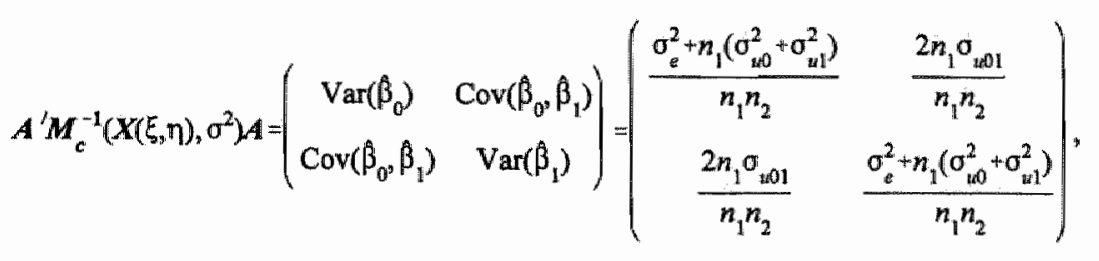

for randomization at the clinic level.

Table 4.5 shows the design criteria, the optimal allocation of units is given in Table 4.6, and the design criteria given the optimal allocation of units are given in Table 4.7. The formulae for the optimal sample sizes and the design criterion given the optimal sample sizes for the $D_{\mathrm{A}}$ - or $D_{S 2}$ - optimality criterion in case of patient level randomization are not given since they are too complex. Instead one may establish the optimal design numerically.

For the $c$ - or $D_{S 1}$ - and $A_{A}$ - optimality criterion the following two conclusions hold: (a) when $r_{x c}{ }^{2}=0$ the value of $\Psi\left(M\left(X(\xi, \eta), \sigma^{2}\right)\right)$ is higher for models with a random treatment effect than for models with a fixed treatment effect; (b) randomization at the patient level is more efficient than randomization at the clinic level also for models with a random treatment effect. For the $D_{A}$ - or $D_{S 2}$ - optimality criterion these two conclusions at least hold when $\sigma_{\mathrm{r} 01}=0$. 
Table 4.5 Design Criteria for both Levels of Randomization and Models with a Random Treatment Effect

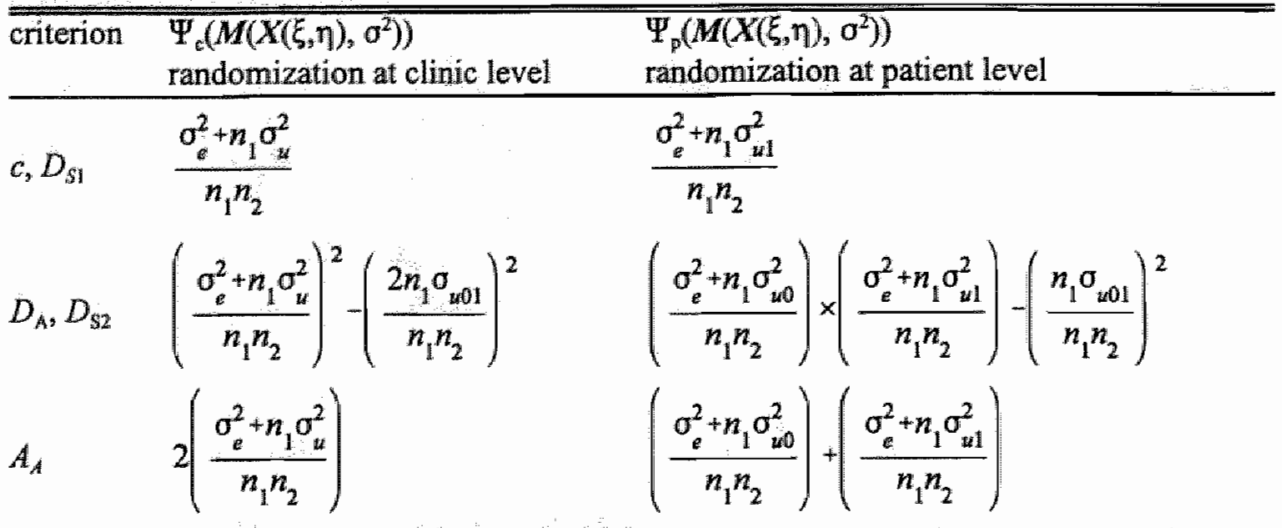

Note. $\quad x_{i j}$ is coded -1 and +1 , and for the covariate $\bar{c}=0$.

$\sigma_{u}^{2}=o_{u 0}^{2}+\sigma_{u 1}^{2}$

Table 4.6 Optimal Sample Sizes for both Levels of Randomization and Models with a Random Treatment Effect

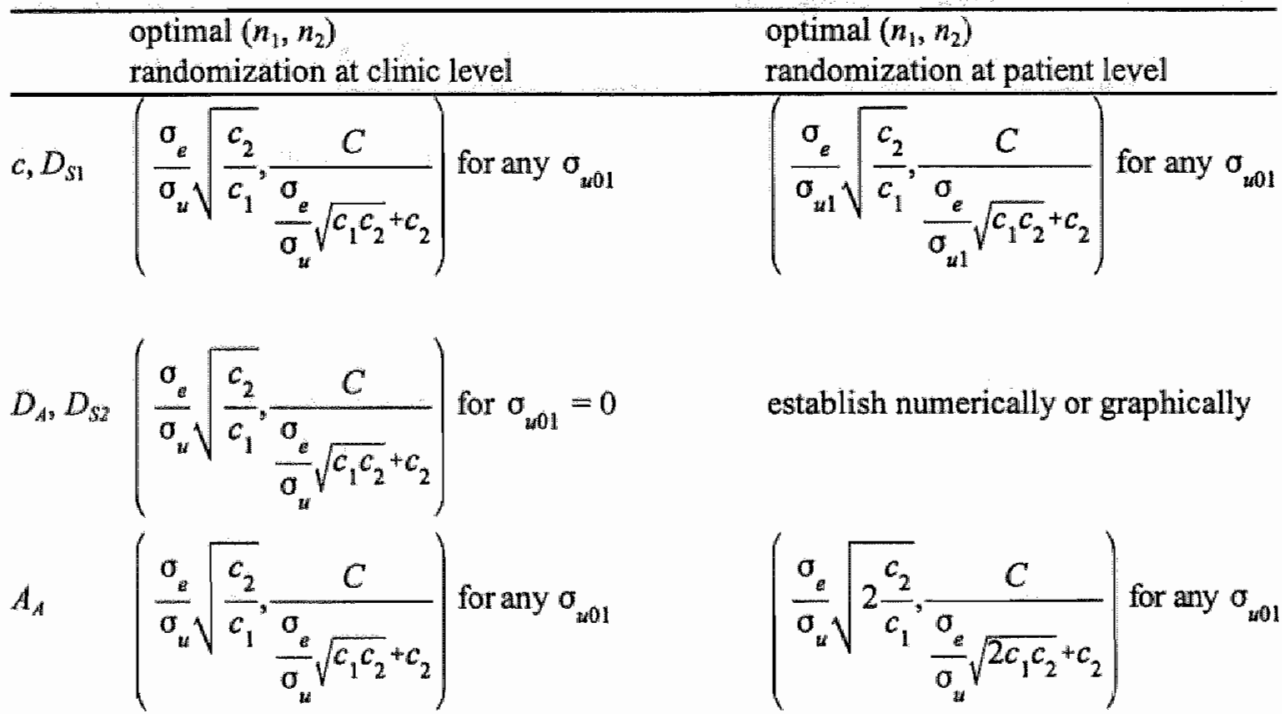

Note. $\quad x_{i j}$ is coded -1 and +1 , and for the covariate $\bar{c}_{\ldots}=0$.

$\sigma_{u}^{2}=\sigma_{u 0}^{2}+\sigma_{u 1}^{2}$

The formulae for the optimal sample sizes for the $D_{A}$ - or $D_{S 2}$ - optimality criterion with randomization the patient level, and for the $D_{A}$ - or $D_{S 2}$ - optimality criterion with $o_{u 01} \neq 0$ for randomization at the clinic level are too complex and not given here. For these cases the optimal design may be established numerically or graphically. 
Table 4.7 Optimal Values of the Design Criterion for both Levels of Randomization and Models with a Random Treatment Effect

\begin{tabular}{|c|c|c|}
\hline & $\begin{array}{l}\Psi_{\mathrm{c}}\left(M\left(\left(X\left(\xi^{*}, \eta\right), \sigma^{2}\right)\right)\right. \\
\text { randomization at clinic level }\end{array}$ & $\begin{array}{l}\Psi_{\mathrm{p}}\left(M\left(X\left(\xi^{*}, \eta\right), 0^{2}\right)\right) \\
\text { randomization at patient level }\end{array}$ \\
\hline$c, D_{51}$ & $\frac{\left(\sigma_{e} \sqrt{c_{1}}+\sigma_{w} \sqrt{c_{2}}\right)^{2}}{C}$ for any $\sigma_{w 01}$ & $\frac{\left(\sigma_{e} \sqrt{c_{1}}+\sigma_{u 1} \sqrt{c_{2}}\right)^{2}}{C}$ for any $\sigma_{* 01}$ \\
\hline$D_{A}, D_{s 2}$ & $\left(\frac{\left(\sigma_{e} \sqrt{c_{1}}+\sigma_{u \sqrt{c_{2}}}\right)^{2}}{C}\right)^{2}$ for $\sigma_{w 01}=0$ & establish numerically or graphically \\
\hline$A_{A}$ & $\frac{\left(\sigma_{e} \sqrt{2 c_{1}}+\sigma_{u} \sqrt{2 c_{2}}\right)^{2}}{C}$ for any $\sigma_{u 01}$ & $\frac{\left(\sigma_{e} \sqrt{2 c_{1}}+\sigma_{u} \sqrt{c_{2}}\right)^{2}}{C}$ for any $\sigma_{w 01}$ \\
\hline
\end{tabular}

Note $\quad x_{i j}$ is coded -1 and +1 , and for the covariate $\bar{c}_{. .}=0$.

$\sigma_{u}^{2}=\sigma_{w 0}^{2}+\sigma_{u !}^{2}$

The formulae for the design criterion given the optimal sample sizes for the $D_{A}$ - or $D_{52}$ optimality criterion with randomization the patient level, and for the $D_{A}-$ or $D_{S 2}$ optimality criterion with $\sigma_{u 01} \neq 0$ for randomization at the clinic level are too complex and not given here. For these cases the optimal design may be established numerically or graphically.

\subsection{Effects on the estimated variance components of excluding covariates from the model with a random treatment effect}

For randomization at the clinic level the change in variance components due to the exclusion of the covariate follows from table 4.4 with $\sigma_{u 0}^{2}$ replaced by $\sigma_{u}^{2}=\sigma_{u 0}^{2}+\sigma_{u \|}^{2}$. For randomization at the patient level the change in variance components due to the exclusion of the covariate can be derived as follows. When the clinic level component of the covariate, $\bar{c}_{m}$ is excluded from the multilevel model (4.18), the change in variance components can be calculated from the following three equations:

1. the variance of the outcomes (see formula (7.10) in Snijders and Bosker, 1999):

$$
\begin{aligned}
& \operatorname{Var}\left(y_{i j}\right)=\operatorname{Var}\left(\hat{\beta}_{1}^{*} x_{i j}+\hat{\beta}_{3}^{*}\left(c_{i j}-\bar{c}_{j}\right)+\hat{u}_{0 j}^{*}+\hat{u}_{1 j}^{*} x_{i j}+\hat{e}_{i j}^{*}\right) \\
& =\hat{\beta}_{1}^{2} \operatorname{var}\left(x_{i j}\right)+\hat{\beta}_{3}^{2} \operatorname{var}\left(c_{i j}-\bar{c}_{i j}\right)+\hat{\alpha}_{u 0^{*}}^{2}+\hat{\alpha}_{u 1}^{2} \cdot \operatorname{var}\left(x_{i j}\right)+\hat{\theta}_{e}^{2} . \\
& =\operatorname{Var}\left(\hat{\beta}_{1} x_{i j}+\hat{\beta}_{2} \bar{c}_{j}+\hat{\beta}_{3}^{*}\left(c_{i j}-\bar{c}_{j}\right)+\hat{u}_{0 j}+\hat{u}_{i j} x_{i j}+\hat{e}_{i j}\right) \\
& =\hat{\beta}_{1}^{2} \operatorname{var}\left(x_{i j}\right)+\hat{\beta}_{2}^{2} \operatorname{var}\left(\bar{c}_{j j}\right)+\hat{\beta}_{3}^{2} \operatorname{var}\left(c_{i j}-\bar{c}_{j}\right)+\hat{\sigma}_{u 0}^{2}+\hat{\sigma}_{u 1}^{2} \operatorname{var}\left(x_{i j}\right)+\hat{\sigma}_{e^{3}}^{2}
\end{aligned}
$$

where $+\sigma_{u 01}$ for $x_{i j}=+1$ cancels against $-\sigma_{u 01}$ for $x_{i j}=-1$. 
2. the difference scores of the average outcomes in the two treatment groups:

$$
\begin{aligned}
\operatorname{Var}\left(\frac{1}{2}\left(\bar{y}_{j t}-\bar{y}_{j l}\right)\right) & =\operatorname{Var}\left(\hat{\beta}_{1}^{*}+\hat{u}_{1 j}^{*}+\frac{1}{2}\left(\hat{\bar{e}}_{j t}-\hat{\bar{e}}_{j k}\right)\right)=\hat{\sigma}_{u 1}^{2}+\frac{\hat{\sigma}_{e}^{2}}{n_{1}} \\
& =\operatorname{Var}\left(\hat{\beta}_{1}+\hat{u}_{1 j}+\frac{1}{2}\left(\hat{\bar{e}}_{j t}-\hat{\bar{e}}_{j k}\right)\right)=\hat{0}_{u 1}^{2}+\frac{\hat{\sigma}_{e}^{2}}{n_{1}}
\end{aligned}
$$

3. the sum scores of the average outcomes in the two treatment groups:

$$
\begin{aligned}
\operatorname{Var}\left(\frac{1}{2}\left(\bar{y}_{j t}+\bar{y}_{j c}\right)\right) & =\operatorname{Var}\left(\hat{\beta}_{0}^{*}+\hat{u}_{0 j}^{*}+\frac{1}{2}\left(\hat{\bar{e}}_{j t}+\frac{\bar{e}_{j k}}{j}\right)=\hat{\sigma}_{w 0}^{2}+\frac{\partial_{e}^{2}}{n_{1}}\right. \\
& =\operatorname{Var}\left(\hat{\beta}_{0}+\hat{\beta}_{2} \bar{c}_{j}+\hat{u}_{0 j}+\frac{1}{2}\left(\hat{\bar{e}}_{j t}+\hat{\bar{e}}_{j c}\right)\right)=\hat{\beta}_{2}^{2} \operatorname{var}\left(\bar{c}_{j}\right)+\delta_{u 0}^{2}+\frac{\hat{\sigma}_{e}^{2}}{n_{1}},
\end{aligned}
$$

where the treatment and control groups are denoted by subscripts $t$ and $c$, respectively. From (4.21) - (4.23) it follows that $\sigma_{w 0^{\circ}}^{2}-\partial_{u 0}^{2}=\beta_{2}^{2} \operatorname{Var}\left(\bar{c}_{j}\right)>0$, and that $\sigma_{u 1}^{2}$ and $\sigma_{e}^{2}$ do not change. This is obvious since the exclusion of a between- clinic covariate cannot change within-clinic variation. Consequently, the variance of the treatment effect estimator as given by (4.19) will not change for patient level randomization when $\bar{c}_{j}$ is excluded from (4.18).

Aggregation to difference or sum scores cannot be used to establish the change in variance components due to the exclusion of the patient level component of the covariate, $c_{i j}-\bar{c}_{j, t}$ This is a consequence of the fact that $c_{i j}-\bar{c}_{j}$ is uncorrelated with $x_{i j,}$ and so has zero mean in each treatment group, in each clinic $j$. It can, however be argued, that only $\sigma_{e}^{2}$ will change due to the exclusion of $c_{i j}-\bar{c}_{j}$ from (4.18). This is due to the fact that a within-clinic covariate cannot change between-clinic variation, so $\sigma_{w 0}^{2}$ will remain unchanged. Furthermore, if $c_{i j}-\bar{c}_{j}$ is uncorrelated with $x_{i j}$ within each clinic $j$, these two predictor variables are orthogonal and so the slopes $\beta_{1 j}=\beta_{1}+w_{y}$ of the within-clinic regression lines relating $y_{i j}$ to $x_{i j}$, and consequently $\sigma_{u 1}^{2}$, will not change.

\subsection{Discussion and conclusions}

From this paper the following conclusions can be drawn for models with a fixed treatment effect. From Table 4.1 it is obvious that elimination of the correlation between treatment condition and covariate by using large sample sizes or by means of pre-stratification on the covariate leads to a more efficient design. Once this correlation is eliminated, the optimal level of randomization is the patient level, regardless of the specific design criterion $\Psi\left(M\left(X(\xi, \eta), \sigma^{2}\right)\right)$ within the set of criteria considered in this paper. Optimal sample sizes given a fixed budget $C$ for measuring and sampling are given in Table 4.2 for models with a fixed 
treatment effect, which show that the optimal sample sizes do not depend on the design criterion if randomization is done at the clinic level. Furthermore, for both levels of randomization a $c$ - optimal design corresponds to a $D_{S 1}$ - optimal design, and a $D_{*}$ - optimal design corresponds to a $D_{S 2}$ - optimal design. The efficiency of randomization at the clinic level versus randomization at the patient level is shown in Figures $4.1,4.2$, and 4.3, from which it follows that randomization at the patient level is especially preferable when the intra-clinic correlation coefficient $\rho_{c}$ or the cost ratio $c_{2} / c_{1}$ is large.

Models with a random treatment effect were discussed in Sections 4.6 and 4.7. For the $c$ - or $D_{S 1}$ - and the $A_{A}$ - optimality criterion the following two conclusions hold: (a) when $r_{x i}^{2}=0$ the value of $\Psi\left(M\left(X(\xi, \eta), \sigma^{2}\right)\right)$ is higher for models with a random treatment effect than for models with a fixed treatment effect; (b) randomization at the patient level is again more efficient than randomization at the clinic level for models with a random treatment effect. For the $D_{A}$ - or $D_{\Omega \Omega}$ - optimality criterion these two conclusions at least hold when $\sigma_{u 01}=0$, see Section 4.6 .

The results in this paper not only hold for models with one covariate, but also for models including more covariates. A more efficient design can be achieved by eliminating the multiple correlation between treatment and the covariates. This may be difficult to achieve by means of pre-stratification, but may be achieved by using large sample sizes (i.e. large $n_{\Downarrow} n_{2}$ for randomization at the patient level, and large $n_{2}$ for randomization at the clinic level).

Summarizing: the patient level is generally the optimal level of randomization for models with a fixed or random treatment effect. Furthermore, for this level of randomization a zero correlation between treatment condition and the covariate is easier to achieve since then this correlation is based on sample size $n_{1} n_{2}$, whereas for randomization at the clinic level it is based on sample size $n_{2}$. If randomization at the patient level is not possible, randomization has to be done at the clinic level which results in a larger budget to obtain a certain efficiency. For randomization at the clinic level the optimal sample sizes do not depend on the chosen design criterion.

\section{References}

Atkinson, A. C., \& Donev, A. N. (1996), Optimum Experimental Designs. Oxford: Clarendon Press. Cochran, W. G. (1983). Planning and Analysis of Observational Studies. New York: Willey.

Donner, A. (1998). Some aspects of the design and analysis of cluster randomization trials, Applied Statistics, 47, 95-113.

Donner, A., Birkett, N., \& Buck, C. (1981). Randomization by cluster. Sample size requirements and analysis, American Joumal of Epidemiology, 114,906-914.

Feng, Z., \& Grizzle, J. E. (1992). Correlated binomial variates: properties of estimator of intraclass correlation and its effect on sample size calculation, Statistics in Medicine, 11, 1607-1614.

Goldstein, H. (1986). Multilevel mixed linear model analysis using iterative generalized least squares. Biometrika, 73, 43-56.

Goldstein, H. (1989). Restricted unbiased iterative generalized least squares estimation. Biometrika 76 , $622-623$. 
Goldstein, H. (1995). Mullitevel Statistical Models (2nd ed.). London: Edward Amold.

Hedeker, D., Gibbons, R. D., \& Watemaux, C. (1999). Sample size estimation for longitudinal designs with attrition: comparing time-related contrasts between groups, Journal of Educational and Behavioral Statistics, 24. 70-93.

Hsieh, F. Y. (1988). Sample size fomulae for intervention studies with the cluster as unit of randomization, Statistics in Medicine, 8, 1195-1201.

Kendall, M., \& Stuart, A. (1979). The Advanced Theory of Slatistics. Volume 2: Inference and relationship (4th ed). London: Griffin.

Lee, E. W., \& Dubin, N. (1994). Estimation and sample size considerations for clustered binary responses, Statistics in Medicine, 13, 1241-1252.

Lipsitz, S. R., \& Parzen, M. (1995). Sample size calculations for non-randomized studies, The Statistician, 44, 81-90.

Liu, G., \& Liang, K.Y. (1997). Sample size calculations for studies with correlated observations, Biometrics, 53, 937-947.

Moerbeek, M., Van Breukelen, G. J. P. \& Berger, M. P. F. (in press). Design issues for experiments in multilevel populations. Journal of Educational and Behavioral Statistics.

Moerbeek, M., Van Breukelen, G. J. P., \& Berger, M. P. F. (1999). Optimal experimental designs for multilevel logistic models. Manuscript submitted for publication.

Neuhaus, J. M., \& Kalbfleisch, J. D. (1998), Between- and within-cluster covariate effects in the anallysis of clustered data, Biometrics, 54, 638-645.

Raudenbush, S. W. (1997). Statistical analysis and optimal design for cluster randomized trials, Psychological Methods, 2, 173-185.

Shih, W. J. (1997). Sample size and power calculations for periodontal and other studies with elustered samples using the method of generalized estimating equations, Biometrical Joumal, 39, 899908.

Snijders, T. A. B., \& Bosker, R. J. (1993). Standard errors and sample sizes for two-level research, Journal of Educational Statistics; 18, 237-259.

Snijiders, T. A. B., \& Bosker, R. J. (1994). Modeled variance in two-level models, Sociological Methods and Research, 22, 342-363.

Snijders, T. A. B., \& Bosker, R. J. (1999). Multilevel analysis: An introduction to basic and advanced multilevel modelling. London: Sage Publications.

Tan, F. E. S . \& Berger, M. P. F. (1999). Optimal allacation of time points for the random effects model, Communtications in Statistics A, 28, 517-540. 


\title{
Chapter 5
}

\section{A comparison of estimation methods for multilevel logistic models}

\begin{abstract}
In this paper a comparison between Penalized Quasi Likelihood (PQL) and estimation by numerical integration is made for the analysis of two-level experimental binary data with two treatment conditions. The comparison between the estimation methods is made for three situations: randomization to treatment conditions at the cluster level, randomization at the person level with treatment by cluster interaction, and randomization at the person level without such interaction. Criteria for comparison are convergence of the estimation process and improper estimates (i.e. unrealistic high point estimates), and criteria concerning the point estimation (bias, variance, and mean squared error) and testing (bias of the point estimates and of the variances as reported by the software) of the treatment effect. The results show that non-convergence occurs more often when estimation is done by numerical integration. This method may also lead to improper estimates. First. order PQL performs best in terms of point estimation of the treatment effect, but should not be used for testing. For the latter purpose second order $\mathrm{PQL}$ is more applicable. One may choose to use likelihood ratio tests instead of the Wald test in case of numerical integration.
\end{abstract}

Key words: Penalized Quasi Likelihood, numericall integration, experimental data, multilevel logistic model, test statistic

Acknowledgments: We wish to thank Donald Hedeker for adjusting his MLXOR program so that the maximum number of iterations can be specified. 


\subsection{Introduction}

During the last fifteen years much research has been done on the development of statistical models, estimation methods, and computer programs for the analysis of multilevel data. Most attention so far has been paid to the analysis of continuous responses, while in many studies these are measured on a binary scale. In clinical trials, for instance, a patient recovers or does not recover after a treatment has taken place, and in school based smoking prevention interventions a pupil smokes or does not after the intervention has been implemented. In this paper a comparison is made between two different widely used estimation methods for the analysis of multilevel binary data: estimation by numerical integration of the likelihood function which performs a numerical approximation to maximum likelihood estimation (Hedeker and Gibbons, 1994; Gibbons and Hedeker, 1996), and estimation by Penalized Quasi Likelihood. Quasi Likelihood methods, such as Marginal Quasi Likelihood (MQL, Breslow and Clayton, 1993; Goldstein, 1991) and Penalized Quasi Likelihood (PQL, Breslow and Clayton, 1993; Goldstein and Rasbash, 1996), are based on linearization of the non-linear logistic multilevel model. As has already been shown by Rodriguez and Goldman (1995), Goldstein (1995), and Goldstein and Rasbash (1996), MQL produces poor parameter estimates, especially when the variance components are sufficiently large to be interesting, and this method is beyond the scope of this paper. The research presented in the current paper extends the research presented in the papers by Rodriguez and Goldman (1995), Goldstein (1995), and Goldstein and Rasbash (1996), the paper by Zhou, Perkins, and Hui (1999), and the results for first order PQL presented by Breslow and Lin (1995). Data sets were simulated for various combinations of true values of the parameters and allocations of units, and estimated by PQL or numerical integration. Several criteria for assessing the effectiveness of the methods are used in this paper. The purpose of the paper is to show how the estimation methods perform in terms of parameter estimation and hypothesis testing. The comparison of the estimation methods is made in the context of experiments with two different treatment conditions, to which randomization may be done at either level of the multilevel data structure. For randomization at the person level, data on both treatment conditions are availlable per cluster, and so the treatment by cluster interaction can be estimated, which is not the case for randomization at the cluster level. So there are three different situations for which the effectiveness of the estimation methods will be assessed: (a) randomization to treatment conditions at the cluster lewel, (b) randomization at the person level with treatment by cluster interaction, (c) randomization at the person level without treament by cluster interaction. The contents of this paper are as follows. In the next section the multilevel logistic model and its estimation procedures are presented. In Section 5.3 the criteria for comparison are given, and in Section 5.4 some attention is paid to convergence and incorrect estimates. Thereafter the comparison is made for the three different situations. 


\subsection{The multilevel logistic model and its estimation}

In multilevel modeling regression equations are formulated for each level of the multilevel data structure, which are then combined into one single equation model. The individual level model, which relates the binary outcome $y_{i j}$ to treatment condition $x_{i j}$, is given by

$$
y_{i j}=\pi_{i j}+e_{i j}=\frac{1}{1+e^{-\left(\beta_{i j}+\beta_{1} x_{i j}\right)}}+e_{i j}
$$

where $\pi_{i j}$ is the probability of a response $y_{i j}=1$, and the random error term $e_{i j}$ has zero mean and variance $\pi_{i j}\left(1-\pi_{i j}\right)$ since the outcome $y_{i j}$ is Bemoulli distributed. The intercept $\beta_{0 j}$ and slope $\beta_{b j}$ may vary randomly and/or as a function of cluster level covariates. We will restrict to models without covariates, thus $\beta_{0 j}=\beta_{0}+u_{0, j}$ and $\beta_{1 j}=\beta_{1}+u_{1 j,}$ where $u_{0 j}$ and $u_{1 j}$ are normally distributed random terms having zero mean and variance $\sigma_{u 0}^{2}$ and $\sigma_{u 1}^{2}$, respectively, and covariance $\sigma_{w 01}$. Substituting into (5.1) yields the single equation model

$$
y_{i j}=\pi_{i j}+e_{i j}=\frac{1}{1+e^{-\left(\beta_{0}+\beta_{1} x_{i j}+u_{0 j}+\mu_{1 j} x_{i j}\right)}}+e_{i j}
$$

The random term $u_{1} x_{i j}$ may be omitted if treatment by cluster interaction is assumed to be absent. For randomization at the cluster level, there is just one treatment condition per cluster and the two variance components cannot be estimated separately.

The likelihood function, conditional upon the fixed and random effects is given by

$$
L(y \mid \beta, u)=\underset{i j}{\Pi}\left(\pi_{i j}\right)^{y^{i}}\left(1-\pi_{i j}\right)^{1-y_{i j}},
$$

where $y, \beta$, and $\boldsymbol{u}$ are vectors containing the responses, regression coefficients, and random terms, respectively. The conditional likelihood has to be multiplied by the density of $\boldsymbol{u}$ and the random effect $\boldsymbol{u}$ has to be integrated out to obtain the unconditional (marginal) likelihood:

$$
L\left(y \mid \beta, \sigma_{w 0^{2}}^{2}, \sigma_{u 1}^{2}\right)=\int L(y \mid \beta, u) f(\boldsymbol{u}) d \boldsymbol{u}
$$

where $f(u)$ is the density of $\boldsymbol{u}$, which in this paper is assumed to be multivariate normal.

The unconditional likelihood function is intractable, but can be approximated by mumerical integration (Hedeker and Gibbons, 1994; Gibbons and Hedeker, 1997) as is done in the computer program MIXOR (Hedeker and Gibbons, 1996). Estimation of the multilevel logistic model can also be done by linearization of the nonlinear probability $\pi_{i j}$ by using a Taylor series expansion around the current estimated regression coefficients $\tilde{\beta}$ and residuals (posterior means) $\hat{u}_{j}$ :

$$
\pi_{i j}=f\left(X_{i j} \beta+u_{j}\right) \approx f(H)+X_{i j}(\beta-\beta) f^{\prime}(H)+\left(u_{j}-u_{j}\right) f^{\prime}(H)+\left(u_{j}-a_{j}\right)^{2} f^{\prime \prime}(H) / 2
$$


where $H=X_{i j} \beta+\hat{u}_{j}$. This method has been referred to as Penalized Quasi Likelihood (PQL) by Breslow and Clayton (1993). For a first order approximation the last term in (5.5) can be omitted. The linearized model can be estimated using Iterative Generalized Least Squares (IGLS, Goldstein, 1986) or Restricted Iterative Generalized Least Squares (RIGLS, Goldstein, 1989). So there are four different PQL methods: first and second order PQL with IGLS or RIGLS. First and second order PQL is implemented in the computer program MLwiN (Goldstein et al, 1998), first order PQL is available in HLM as well (Bryk, Raudenbush, and Congdon, 1996).

\subsection{Criteria for comparison}

\subsubsection{Criteria concerning point estimation}

We will mainly restrict ourselves to the treatment effect $\beta_{1}$ since this is generally of main interest in experiments. The criteria include the bias, sampling variance, and mean squared error (MSE) of the treatment effect estimator. For each combination of parameter values and allocation of units 200 data sets were generated and estimated. The $\operatorname{Bias}\left(\hat{\beta}_{1}\right)$ is then estimated by

$$
\operatorname{Bias}\left(\hat{\beta}_{1}\right)=\left[\left(\sum_{j=1}^{200} \hat{\beta}_{1 j} / 200\right)-\beta_{1}\right]
$$

where $\beta_{1 j}$ is the estimation of the $\beta_{1}$ obtained from the $j$ th simulated data set. The Sampling Variance $\left(\beta_{1}\right)$ is estimated by the variance of the 200 estimated $\beta_{1 j}$ "s:

$$
\text { Sampling Variance }\left(\hat{\beta}_{1}\right)=\left\{\sum_{j=1}^{200}\left[\hat{\beta}_{1 j}-\left(\sum_{j=1}^{200} \hat{\beta}_{1 j} / 200\right)\right]^{2}\right\} / 199 \text {. }
$$

The $\operatorname{MSE}\left(\hat{\beta}_{1}\right)$ is the sum of the squared $\operatorname{Bias}\left(\hat{\beta}_{1}\right)$ and the Sampling Variance $\left(\hat{\beta}_{1}\right)$ and may be used as the most general criterion concerning point estimation. Ideally the $\operatorname{Bias}\left(\hat{\beta}_{1}\right)$, Sampling Variance $\left(\hat{\beta}_{1}\right)$, and $\operatorname{MSE}\left(\hat{\beta}_{1}\right)$ are as small as possible given the design and true parameter values.

\subsubsection{Criteria concerning testing of the treatment effect}

The null hypothesis $\mathrm{H}_{0}: \beta_{1}=\beta_{1,0}$ may be tested with the statistic

$$
t=\frac{\hat{\beta}_{1}-\beta_{1,0}}{\sqrt{\operatorname{Vâr}\left(\beta_{1}\right)}},
$$

where $\operatorname{Var}\left(\beta_{1}\right)$ is the estimated variance as reported by the software. It may be assumed that this test statistic has the standard normal distribution under the null hypothesis, and this 
assumption is implemented in the software programs MXOR and MLwiN. This assumption, however, only holds when the treatment effect and its variance as reported by the softwaire are estimated unbiasedly, and the $\operatorname{Bias}\left(\hat{\beta}_{1}\right)$ and Variance $\operatorname{Ratio}\left(\hat{\beta}_{1}\right)$ may be used for checking these conditions. The Variance $\operatorname{Ratio}\left(\hat{\beta}_{1}\right)$ is equal to the ratio of the Sampling Variance $\left(\beta_{1}\right)$ and the mean of the $\operatorname{Var}\left(\hat{\beta}_{1}\right)$ as reported by the software, and measures the unbiasedness of these reported $\operatorname{Var}\left(\hat{\beta}_{1}\right)$. Ideally the $\operatorname{Variance} \operatorname{Ratio}\left(\hat{\beta}_{1}\right)$ is equal to one.

\subsection{Convergence and behavior of the estimation procedure}

For the model with person level randomization (i.e. $\frac{1}{2} n_{1}$ persons per treatment condition per cluster) and a fixed slope and for cluster level randomization (i.e. $\frac{1}{2} n_{2}$ clusters per treatment condition) data sets were generated for the multilevel model in (5.2) without a random slope for the following true parameter values and number of allocated units: $\beta_{0}=0$, $\beta_{1}=0,0.5,1$, or $1.5, \sigma^{2}=0,0.25,0.5$, or 1 , and $\left(n_{1}, n_{2}\right)=(40,10),(20,20)$, and $(10,40)$. So there were 48 combinations of true parameter values and allocations of units, which will be called simulation conditions, and for each of these conditions 200 data sets were generated. For the model with person level randomization and a random slope the same allocations of units and the same parameter values for the intercept, slope, and intercept variance were used, combined with two different values for the slope variance: $\sigma_{u 1}^{2}=0$ or 0.25 , and a correlation between intercept and slope equal to $\sigma_{u 01}=0_{n}$ Larger values of $\sigma_{u 1}^{2}$ would be too large compared with $\sigma_{w 0}^{2}$ and were not considered in this simulation study. So there were 96 simulation conditions for which 200 data sets were generated. Given $\beta_{1}>0$ and $x_{b j}$ coded as -1 and +1 for the control and treatment group, respectively, the range of the probability $\pi_{i j}$ of a response $y_{i j}=\mathbb{1}$ is approximately

$$
\text { range } \pi_{i j}=\left(\left[1+\exp \left(-\beta_{0}+\beta_{1}+2 \sigma_{w 0}+2 \sigma_{w 1}\right)\right]^{-1},\left[1+\exp \left(-\beta_{0}-\beta_{1}-2 \sigma_{w 0}-2 \sigma_{w 1}\right)\right]^{-1}\right)
$$

and so a wide range for the probability $\pi_{i j}$ is covered by our simulation conditions. For example if $\beta_{0}=0, \beta_{1}=1.5, \sigma_{u 0}^{2}=1$, and $\sigma_{w \|}^{2}=0.25$ then the range for $\pi_{i j}=(0.01,0.99)$; and for $\beta_{0}=0, \beta_{1}=0, \sigma_{u 0}^{2}=0$, and $\sigma_{u \mathbb{I}}^{2}=0$ the range is $(0.5,0.5)$.

Estimation by numerical integration was done using MLXOR with a maximum of 1000 itterations, a convergence criterion of 0.001 for the absolute change in parameter estimates, and 10 quadrature points as was suggested by Hedeker and Gibbons (1996). MLwiN with a maximum of 100 iterations and a convergence criterion of 0.001 for the relative change in parameter estimates was used for PQL estimation.

Especially for small values of the variance components the estimation process did not always converge. For estimation by numerical integration non-convergence occurred for at most $60 \%$ of the generated data sets of a given simulation condition. For PQL estimation it occurred for up to $5 \%$ of the generated data sets for models with a fixed slope and mainly if 
$\sigma^{2}=0$. For models with a random slope first order PQL did not converge for all most $10 \%$ of the generated data sets of a given simulation condition, whereas second order PQL did not converge for at most $27.5 \%$ of the generated data sets and for this method the number of data sets for which non-convergence occurred increases if $\sigma_{w 0}^{2}$ increases and if $\sigma_{w 1}^{2}$ decreases. The estimates from the last iteration were taken when the estimation process did not converge, except when these were evidently incorrect.

For some data sets MIXOR did not produce output, or converged to an evidently incorrect solution, which is defined here as a solution for which one or both of the variance components are greater than 3, or fitted a model with one less random effect. For models with a fixed slope this especially occurred for small $\sigma_{w 0}^{2}$ but seldom for more than $10 \%$ of the generated data sets of a given simulation condition. The estimates of the model with one less random effect (i.e. the model with a fixed intercept) were used instead since fitting a model with one random effect less is the most logical thing to do in practice, and sometimes this is automatically done by MIXOR as well. On the other hand such problems occurred considerably more often for models with a random slope: for at most $90 \%$ of the generated. data sets of a given simulation condition when $\sigma_{u 1}^{2}=0$, and for at most $70 \%$ when $\sigma_{u I}^{2}=0.25$. Since these percentages were so large we only considered the generated data sets for which such estimation problems did not occur to make a fair comparison with $\mathrm{PQL}$ estimation.

\subsection{Randomization at person level and assuming no treatment by cluster interaction}

\subsubsection{Criteria concerning point estimation}

Bias. From Figure 5.1 it follows that the treatment effect is generally overestimated by second order PQL or numerical integration and generally underestimated by first order PQL, except when $\sigma_{w 0}^{2}=0$. The $\operatorname{Bias}\left(\beta_{1}\right)$ is smaller than $10 \%$ of the true $\beta_{1}\left(\right.$ for $\beta_{1}>0$ ) for first order $\mathrm{PQL}$. For second order $\mathrm{PQL}$ and estimation by numerical integration it is even smaller than $5 \%$, and generally the smallest $B i a s\left(\beta_{1}\right)$ is obtained by numerical integration. Furthermore, the absolute $\operatorname{Bias}\left(\beta_{1}\right)$ increases with $\beta_{1}$ (given $\beta_{0}=0$ ) and $\sigma_{w 0}^{2}$ for all estimation methods, and with $n_{2}$ (given $n_{1} n_{2}=400$ ) for first order PQL as well. The relative Bias $\left(\beta_{1}\right)$, however, does not depend on $\beta_{1}$.

Sampling Variance and MSE. Figure 5.1 shows that the Sampling Variance $\left(\hat{\beta}_{1}\right)$ increases with $\beta_{1}$ (given $\beta_{0}=0$ ), but not with $\sigma^{2}$ (see also Moerbeek, Van Breukelen, and Berger, 1999). Generally the smallest Sampling Variance $\left(\beta_{1}\right)$ is obtained by first order PQL. The $\operatorname{MSE}\left(\hat{\beta}_{1}\right)$ resembles the Variance $\operatorname{Ratio}\left(\hat{\beta}_{1}\right)$, except that there is less difference between the estimation methods since first order $P Q L$ has larger bias. The smallest $\operatorname{MSE}\left(\beta_{1}\right)$ is obtained by first order PQL and so this method performs best in terms of point estimation of $\beta_{1}$, although the difference in MSE among the estimation methods is not really large. 

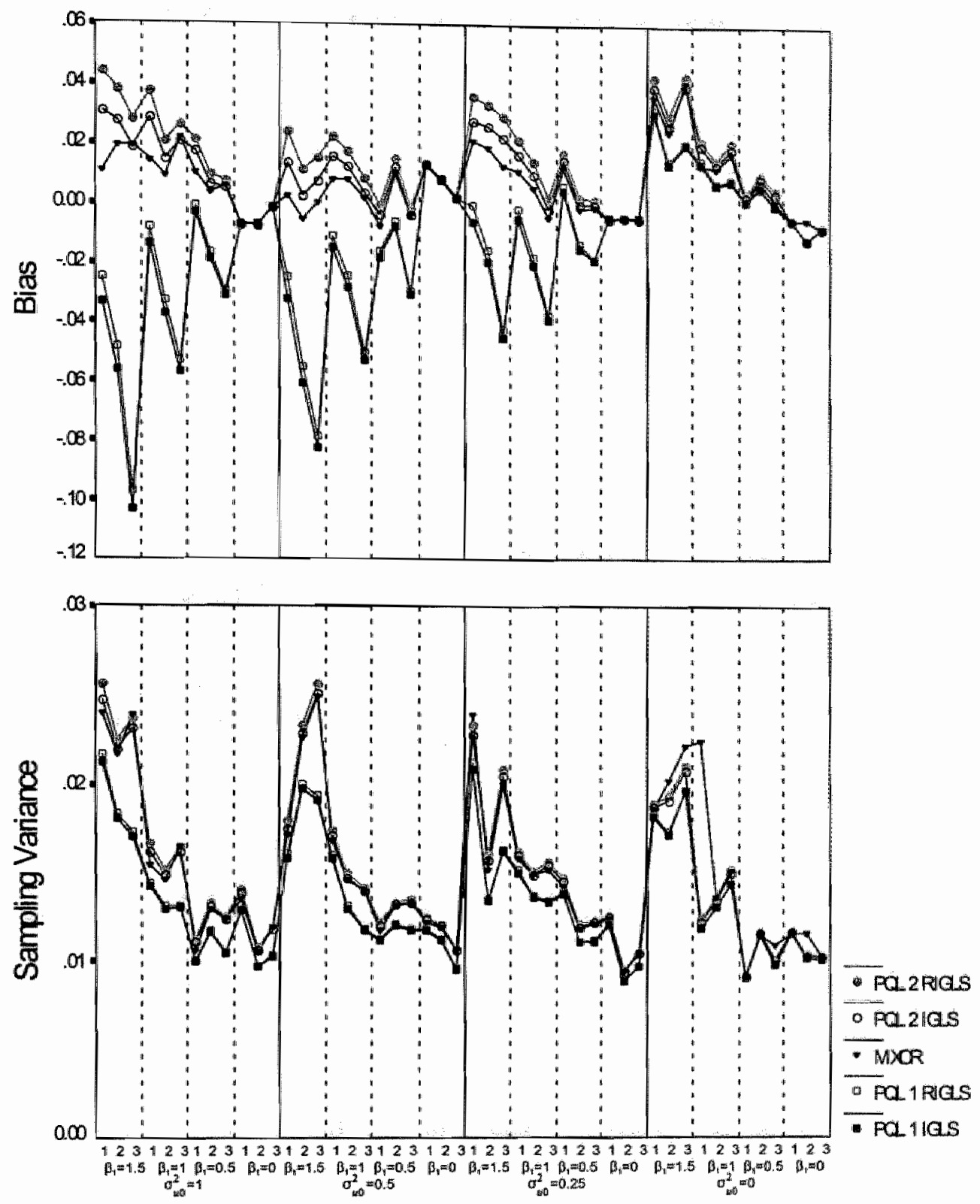

Figure 5.1. Bias $\left(\beta_{1}\right)$ and Sampling $\operatorname{Variance}\left(\beta_{1}\right)$ as a function of the simulation conditions for a model with randomization at the person level and a fixed slope. Allocations of units are denoted ' $^{\prime}=\left(n_{1}, n_{2}\right)=(40,10), 2^{\prime}=\left(n_{1}, n_{2}\right)=(20,20), 3^{\prime}=\left(n_{1}, n_{2}\right)=(10,40)$. 


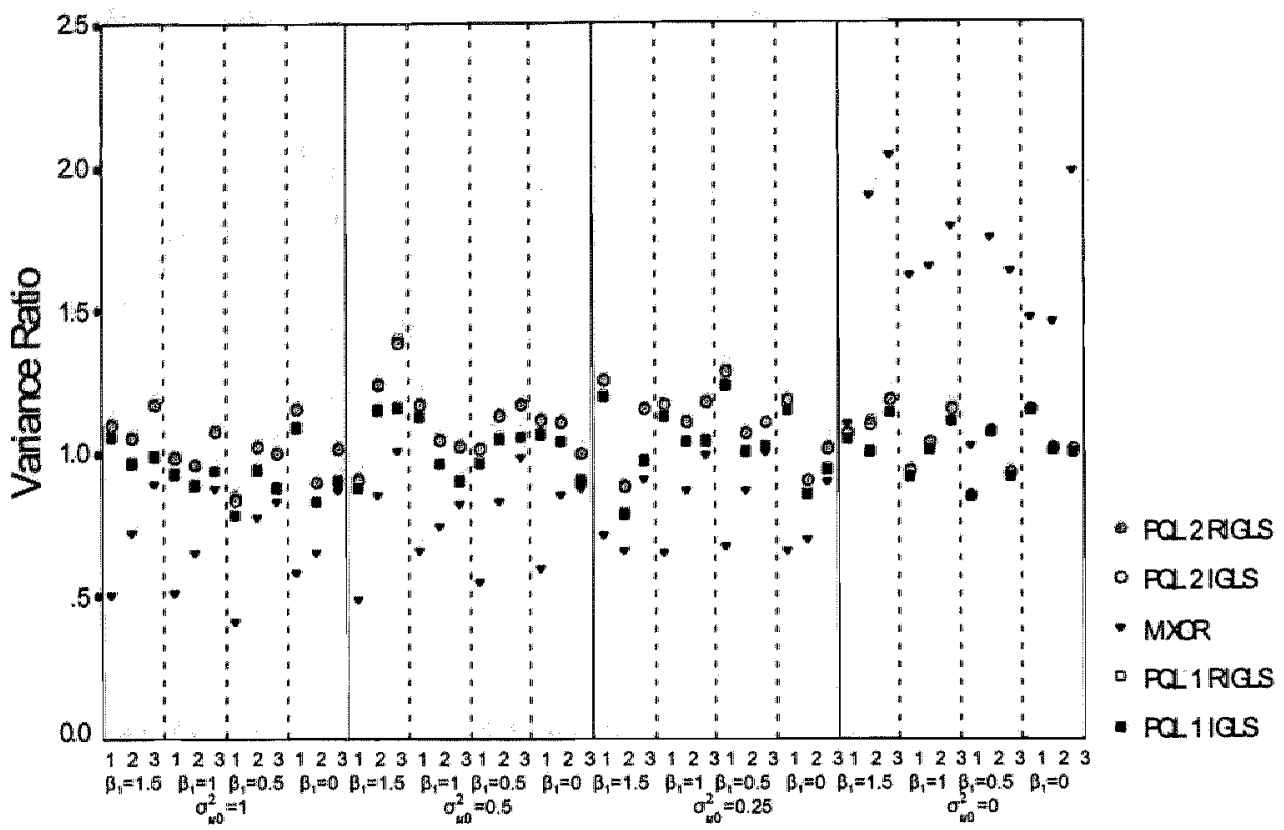

Figure 5.2. Variance Ratio $\left(\hat{\beta}_{1}\right)$ as a function of the simulation conditions for a model with randomization at the person level and a fixed slope. Allocations of units are denoted $' 1^{\prime}=\left(n_{1}, n_{2}\right)=(40,10), 2^{\prime}=\left(n_{1}, n_{2}\right)=(20,20), 3^{\prime}=\left(n_{1}, n_{2}\right)=(10,40)$.

Other parameters. The best estimates for the intercept $\beta_{0}$ in terms of MSE were produced by first order $\mathrm{PQL}$, although there was not much difference between the estimation methods, and for all methods the absolute bias was seldom larger then 0.02. First order PQL also produced the best estimates in terms of MSE for the intercept variance $\sigma_{u 0}^{2}$, but again there was not much difference between the methods. For all methods the relative bias did not depend on $\sigma_{u 0}^{2}$ and was seldom larger than $20 \%$ of the true $\sigma_{u 0}^{2}\left(\right.$ for $\left.\sigma_{w 0}^{2}>0\right)$.

\subsubsection{Criteria concerning testing of the treatment effect}

The Variance Ratio $\left(\beta_{1}\right)$, which is equal to the Sampling Variance $\left(\hat{\beta}_{1}\right)$ divided by $\operatorname{Var}\left(\hat{\beta}_{1}\right)$ as given by the software, is given in Figure 5.2, showing that the reported $\operatorname{Var}\left(\hat{\beta}_{1}\right)$ are almost unbiased when PQL estimation is used. For first order PQL estimation $\beta_{1}$ itself is biasedly estimated, and consequently the mean of the test statistic (5.8) is shifted from zero. This is shown for the simulation condition $\beta_{1}=1, \sigma_{u 0}^{2}=0.5,\left(n_{1}, n_{2}\right)=(20,20)$ by the probability plot in Figure 5.3, the deviation from the standard normal distribution will be more or less serious for small or large $n_{2}$, respectively. So the standard normal distribution 

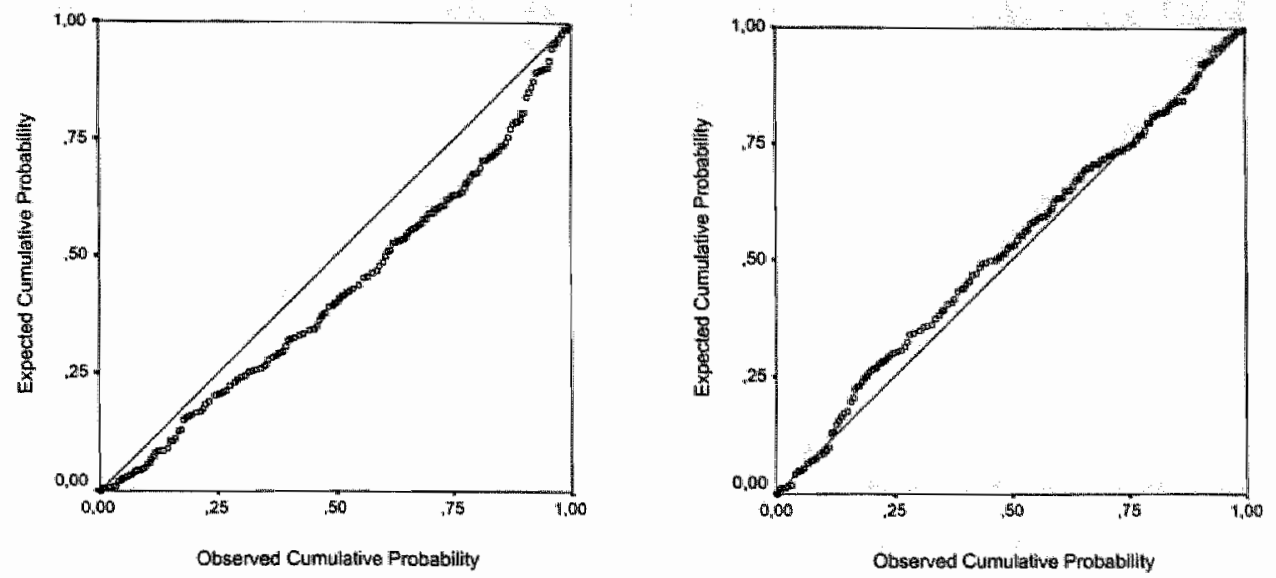

Figure 5.3. Standard normal probability plot for the test statistics produced by first order PQL with IGLS (left), and for second order PQL with IGLS (right) for the simulation condition $\beta_{1}=1, \sigma^{2}=0.5,\left(n_{1}, n_{2}\right)=(20,20)$
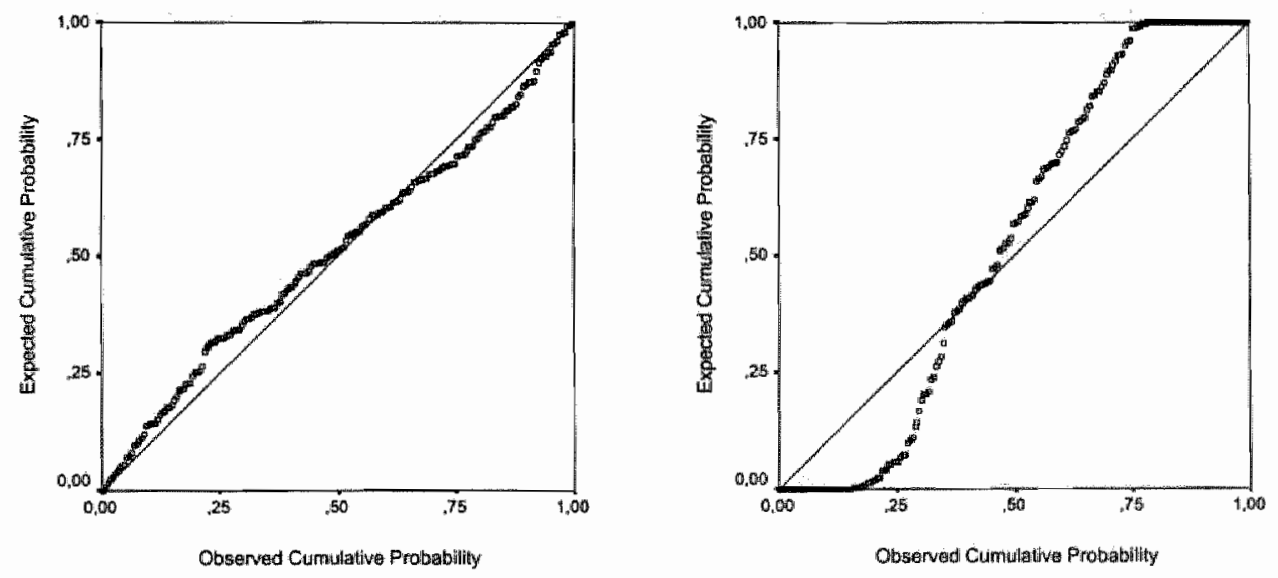

Figure 5.4. Standard normal probability plot for the test statistics produced by MIXOR for the simulation condition $\beta_{1}=1, \sigma^{2}=0.5,\left(n_{1}, n_{2}\right)=(20,20)$ (left), and for the simulation. condition $\beta_{1}=1, \sigma_{w 0}^{2}=0,\left(n_{1}, n_{2}\right)=(20,20)$ (right) 
should not be used as a reference distribution for testing the treatment effect for this estimation method. On the other hand the deviation from the standard normal distribution is small when second order PQL estimation is used since this method produces almost unbiasedly estimated treatment effects, as is also shown for the simulation condition $\beta_{1}=1$, $\sigma_{u t}^{2}=0.5,\left(n_{1}, n_{2}\right)=(20,20)$ by the probability plot in Figure 5.3. Almost unbiased estimates of the treatment effect are obtained by estimation by numerical integration as well, but this method overestimates $\operatorname{Var}\left(\hat{\beta}_{1}\right)$ for $\sigma_{u 0}^{2}>0$ since the $\operatorname{Variance} \operatorname{Ratio}\left(\hat{\beta}_{1}\right)<1$. So the test statistic (5.8) has a mean close to zero but too short tails which results in type II errors, and this especially occurs when $n_{2}=10$ and $n_{1}=40$. Figure 5.4 shows the probability plot for the simulation condition $\beta_{1}=1, \sigma_{u 0}^{2}=0.5,\left(n_{1}, n_{2}\right)=(20,20)$, the deviation from the standard normal distribution is larger for small $n_{2}$. On the other hand the $\operatorname{Var}\left(\hat{\beta}_{1}\right)$ is underestimated when $\sigma_{u 0}^{2}=0$ since then Variance Ratio $\left(\hat{\beta}_{1}\right)>1$, resulting in too long tails and consequently type I errors (see the probability plot in Figure 5.4).

\subsection{Randomization at cluster level}

\subsubsection{Criteria concerning point estimation}

Bias. The treatment effect is generally overestimated by second order PQL and numerical integration, and generally underestimated by first order PQL except when $\sigma_{w 0}^{2}=0$, see Figure 5.5. The Bias $\left(\hat{\beta}_{1}\right)$ was smaller than $10 \%$ of the true $\beta_{1}\left(\beta_{1}>0\right)$ for first order PQL, and even smaller than $5 \%$ of the true $\beta_{1}$ for second order PQL and numerical integration. Furthermore, the absolute $\operatorname{Bias}\left(\hat{\beta}_{1}\right)$ increases with $\beta_{1}$ and $\sigma_{v 0}^{2}$ for all estimation methods, but there is no strong relationship with $n_{2}$. The relative bias only increases with $\sigma_{w 0}^{2}$.

Sampling Variance and MSE. The Sampling Variance $\left(\hat{\beta}_{1}\right)$ not only increases with $\beta_{1}$ (given $\beta_{0}=0$ ), but also with $\sigma_{u 0}^{2}$ and $n_{1}$ (given $n_{1} n_{2}=400$ ) (see also Moerbeek, Van Breukelen and Berger, 1999). Largest Sampling Variance $\left(\hat{\beta}_{1}\right)$ is obtained by estimation by numerical integration, but for small values of $\sigma_{u 0}^{2}$ there is hardly any difference between the estimation methods. The squared $\operatorname{Bias}\left(\hat{\beta}_{1}\right)$ is small compared with the Sampling Variance $\left(\hat{\beta}_{1}\right)$, and therefore the $\operatorname{MSE}\left(\hat{\beta}_{1}\right)$ is almost equal to the latter. So in terms of point estimation first order PQL performs best, although the differences among the estimation methods are small.

Other Parameters. In terms of MSE first order PQL yielded the best estimates for the intercept $\beta_{0}$ and $\sigma_{w 0}^{2}$ as well, but again the differences between the estimation methods were small. The absolute bias for the intercept was seldom larger than 0.2 , whereas the relative bias for $\sigma_{u 0}^{2}$ may be as large as $40 \%$ of the true $\sigma_{u 0}^{2}\left(\right.$ for $\sigma_{u 0}^{2}>0$ ). 

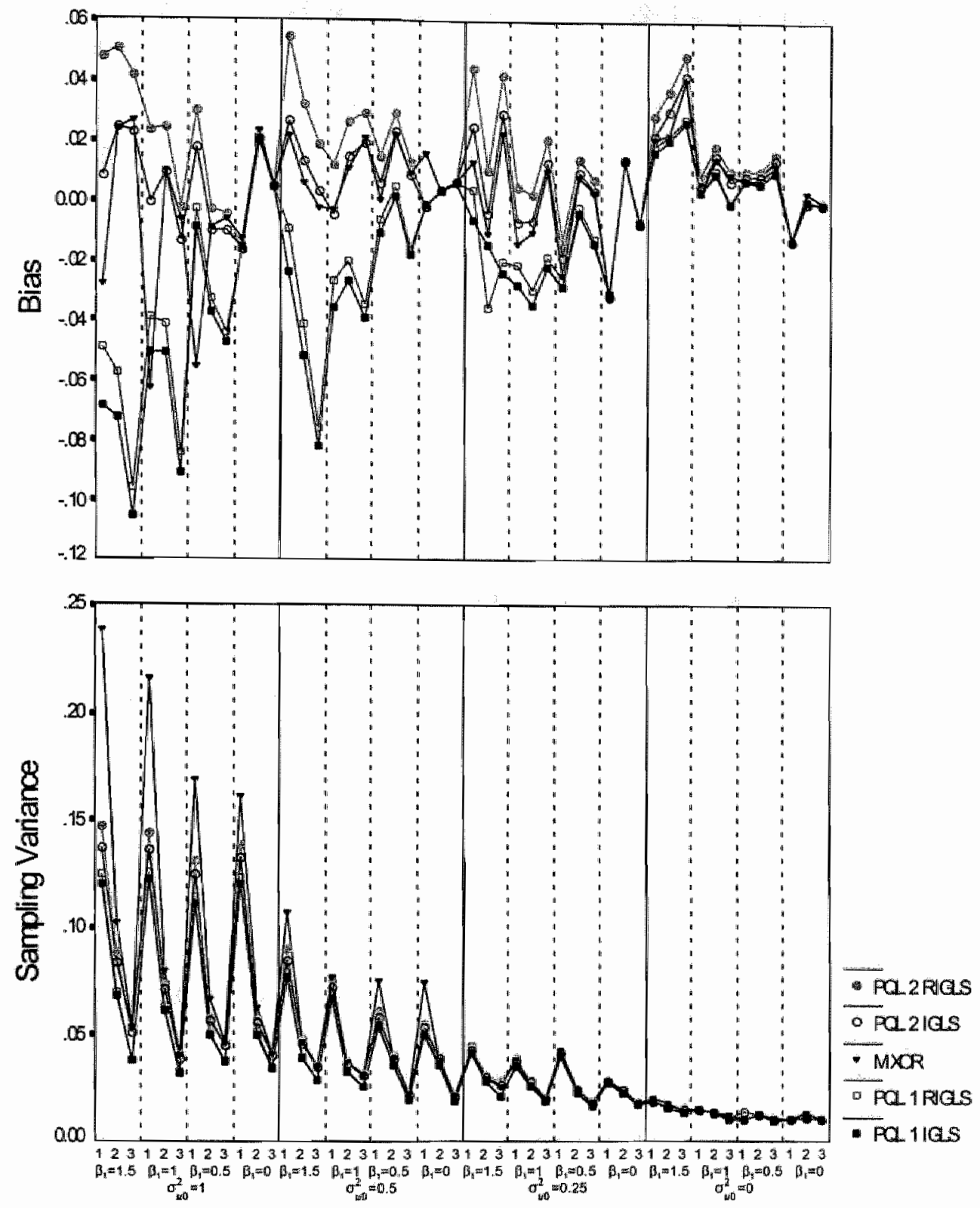

Figure 5.5. Bias $\left(\hat{\beta}_{1}\right)$ and Sampling Variance $\left(\hat{\beta}_{1}\right)$ as a function of the simulation conditions for a model with randomization at the cluster level. Allocations of units are denoted ${ }^{\prime} 1^{\prime}=\left(n_{1}, n_{2}\right)=(40,10), 2^{\prime}=\left(n_{1}, n_{2}\right)=(20,20), 3^{\prime}=\left(n_{1}, n_{2}\right)=(10,40)$. 


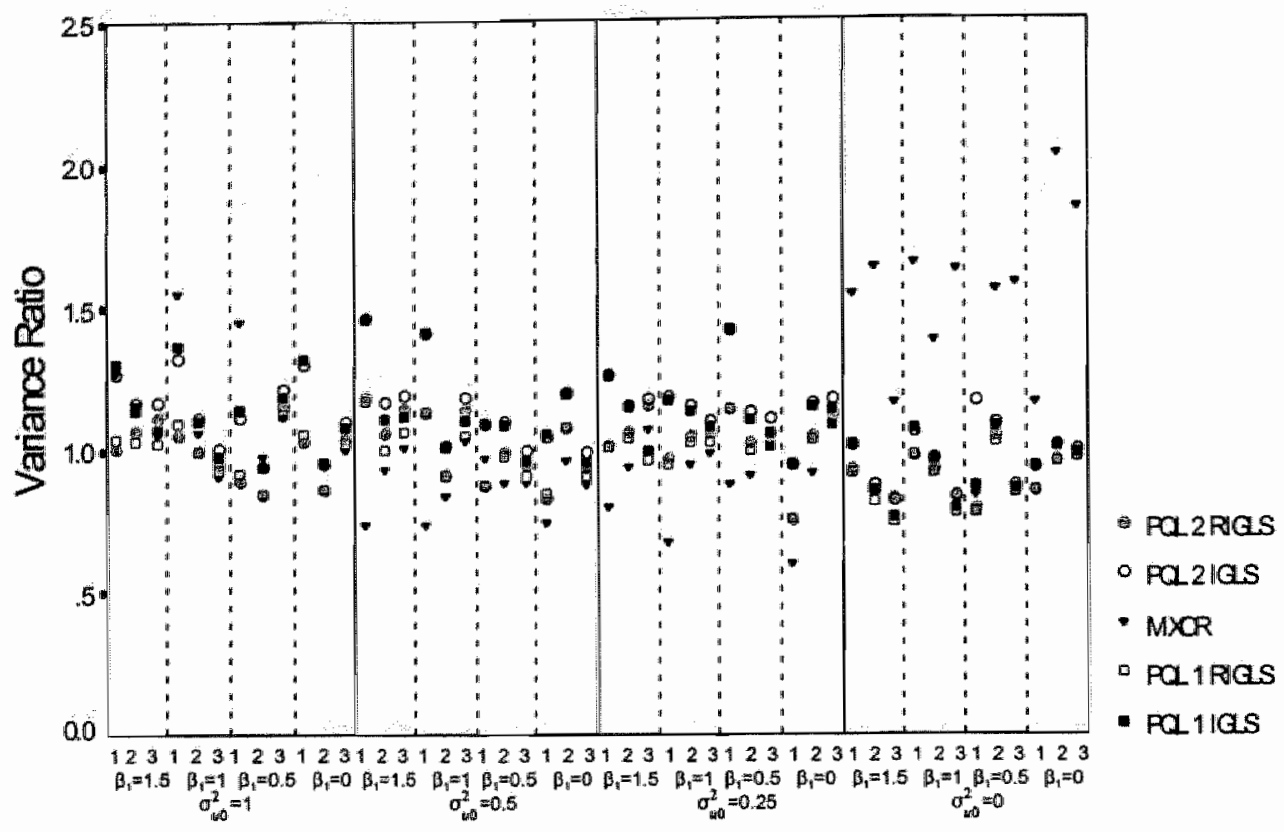

Figure 5.6. Variance Ratio $\left(\beta_{1}\right)$ as a function of the simulation conditions for a model with randomization at the cluster level. Allocations of units are denoted ' 1 ' $=\left(n_{1}, n_{2}\right)=(40,10)$, $2^{\prime}=\left(n_{1}, n_{2}\right)=(20,20), 3^{\prime}=\left(n_{1}, n_{2}\right)=(10,40)$.

\subsubsection{Criteria concerning testing of the treatment effect}

For estimation by numerical integration the $\operatorname{Bias}\left(\hat{\beta}_{1}\right)$ is very small, but as follows from Figure 5.6 the Variance Ratio $\left(\beta_{1}\right)$ is larger than one for $\sigma_{u 0}^{2}=0$ or 1. Consequently the $\operatorname{Var}\left(\beta_{1}\right)$ as reported by the software is too small resulting in too long tails for the distribution of the test statistic (5.8). On the other hand a too large $\operatorname{Var}\left(\hat{\beta}_{1}\right)$ and therefore too short tails are obtained for $\sigma_{u 0}^{2}=0.25$ or 0.5 , but the deviation from the standard normal distribution is not as serious as for randomization at the person level without treatment by cluster interaction. For second order $\mathrm{PQL}$ estimation both the treatment effect and its variance are estimated almost unbiasedly and the standard normal distribution may be used as a reference distribution for testing the treatment effect. For first order $\mathrm{PQL}$ estimation the mean of the test statistic (5.8) is shifted from zero since the treatment effect is biasedly estimated by this method. These conclusions also followed from the normal probability plots that we made for the 48 simulation conditions and the five estimation methods, these plots are similar to those given in Section 5.5 .2 for randomization at the pupil level and no treatment by cluster interaction. 


\subsection{Randomization at person level and assuming treatment by cluster interaction}

\subsubsection{Criteria concerning point estimation}

Bias. The underestimation for first order PQL is seldom larger than $10 \%$ of the true $\beta_{1}$ $\left(\beta_{1}>0\right)$, whereas second order PQL and estimation by numerical integration seldom produce an overestimation of more than $5 \%$. For first order PQL the absolute bias increases with $\beta_{1}$ (given $\beta_{0}=0$ ), $\sigma_{t 0}^{2}, \sigma_{u 1}^{2}$, and $n_{1}$ (given $n_{1} n_{2}=400$ ), whereas the relative bias does not depend on $\beta_{1}$. For second order PQL and estimation by numerical integration there was no strong relationship between the bias and parameter values or number of allocated units. See Figures 5.7 and 5.8 for more details.

Sampling Variance and MSE. For all estimation methods the Sampling Variance $\left(\hat{\beta}_{1}\right)$ increases with $\beta_{1}$ (given $\beta_{0}=0$ ) and $\sigma_{u 1}^{2}$. For $\sigma_{u 1}^{2}>0$ it increases with $n_{11}$ as well (given $n_{1} n_{2}=400$ ), see Figures 5.7 and 5.8 (and also Moerbeek, Van Breukelen, and Berger, 1999). Generally the smallest Sampling Variance $\left(\beta_{1}\right)$ is obtained by first order PQL estimation. The $\operatorname{MSE}\left(\hat{\beta}_{1}\right)$ resembles the Variance Ratio $\left(\hat{\beta}_{1}\right)$ except that the difference between the estimation methods is smaller since first order PQL has largest squared bias, but this method still has the lowest $\mathrm{MSE}\left(\beta_{1}\right)$.

Other parameters. The smallest MSE for estimating the variance components is obtained with first order PQL as well, but again there is not much difference between the estimation methods.

\subsubsection{Criteria concerning testing of the treatment effect}

For all estimation methods the Variance Ratio $\left(\beta_{1}\right)$ is given in Figures 5.9 and 5.10 . For first and second order PQL estimation the Variance Ratio $\left(\hat{\beta}_{1}\right)$ is close to one, but the mean of the distribution for the test statistic (5.8) is shifted from zero when first order PQL is used. For estimation by numerical integration the $\operatorname{Var}\left(\beta_{1}\right)$ as produced by the software is generally underestimated for $\sigma_{w 0}^{2}=0$, leading to too long tails for the distribution of the test statistic (5.8). On the other hand, too short tails occur when $\sigma_{w 0}^{2}>0$ and especially when $n_{2}=10$ and $n_{1}=40$, since then the $\operatorname{Var}\left(\hat{\beta}_{1}\right)$ as produced by the software is generally overestimated. These conclusions also followed from the normal probability plots we made for the 96 simulation conditions and five estimation methods, these plots are similar to those given in Section 5.5 .2 for randomization at the pupil level and no treatment by cluster interaction. 

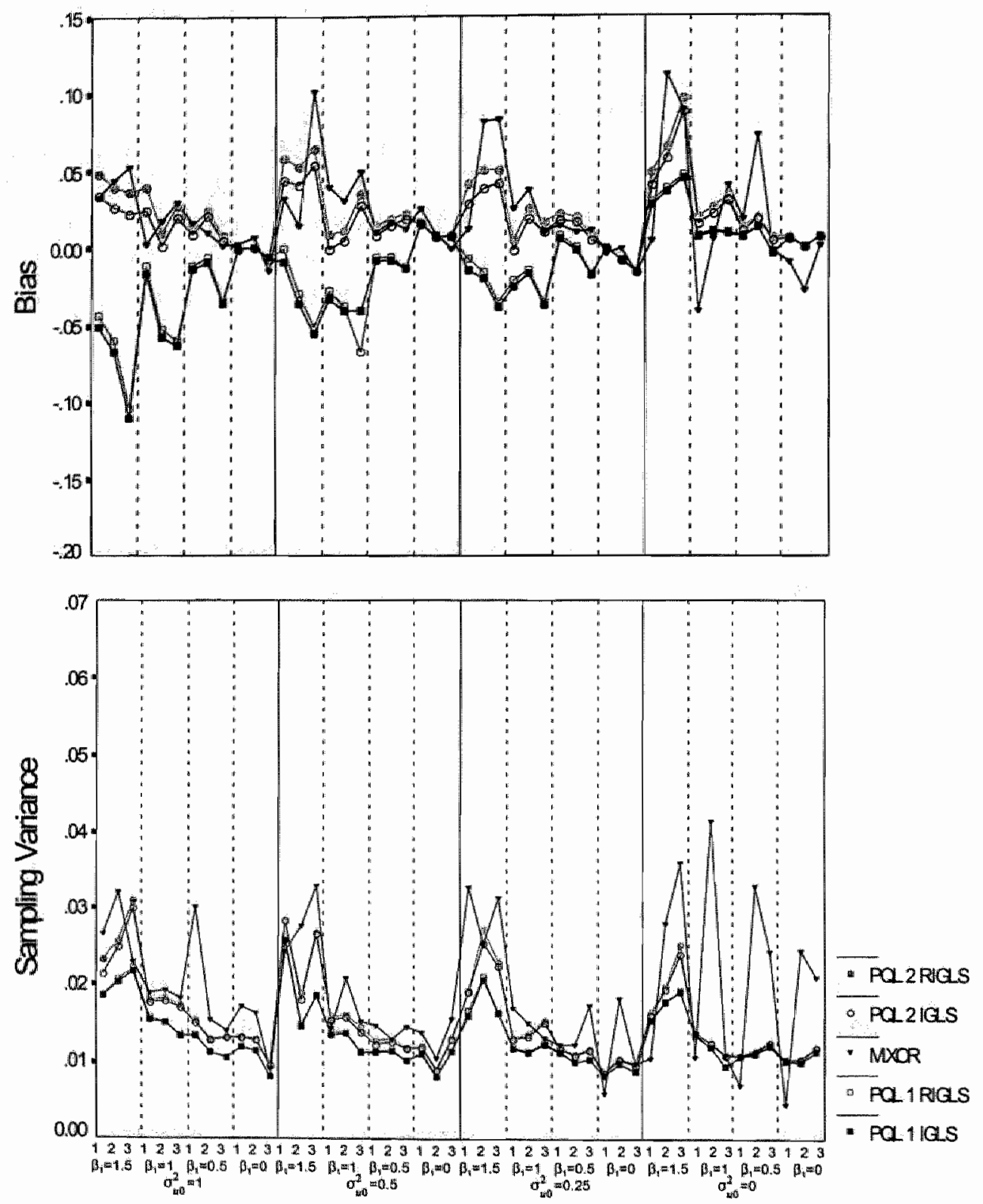

Figure 5.7. Bias $\left(\beta_{1}\right)$ and Sampling Variance $\left(\hat{\beta}_{1}\right)$ as a function of the simulation conditions for a model with randomization at the person level and a random slope with true $\sigma_{u 1}^{2}=0$. Allocations of units are denoted '1' $=\left(n_{1}, n_{2}\right)=(40,10), 2^{\prime}=\left(n_{1}, n_{2}\right)=(20,20), 3^{\prime}=\left(n_{1}, n_{2}\right)=$ $(10,40)$. 

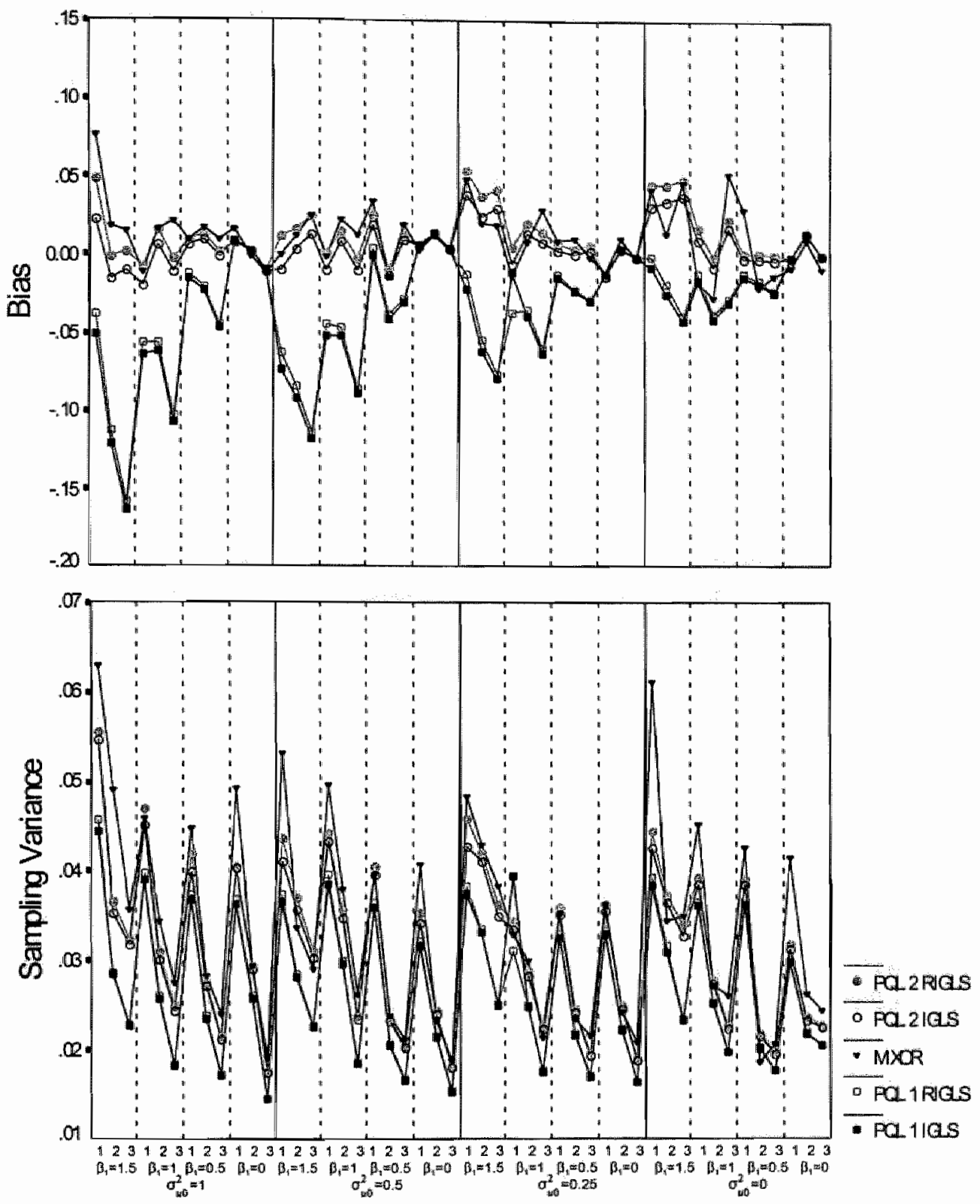

Figure 5.8. Bias $\left(\hat{\beta}_{1}\right)$ and Sampling Variance $\left(\hat{\beta}_{1}\right)$ as a function of the simulation conditions for a model with randomization at the person level and a random slope with $\sigma_{u 1}^{2}=0.25$. Allocations of units are denoted ' 1 ' $=\left(n_{1}, n_{2}\right)=(40,1.0), 2^{\prime}=\left(n_{1}, n_{2}\right)=(20,20), 3^{\prime}=\left(n_{1}, n_{2}\right)=$ $(10,40)$. 


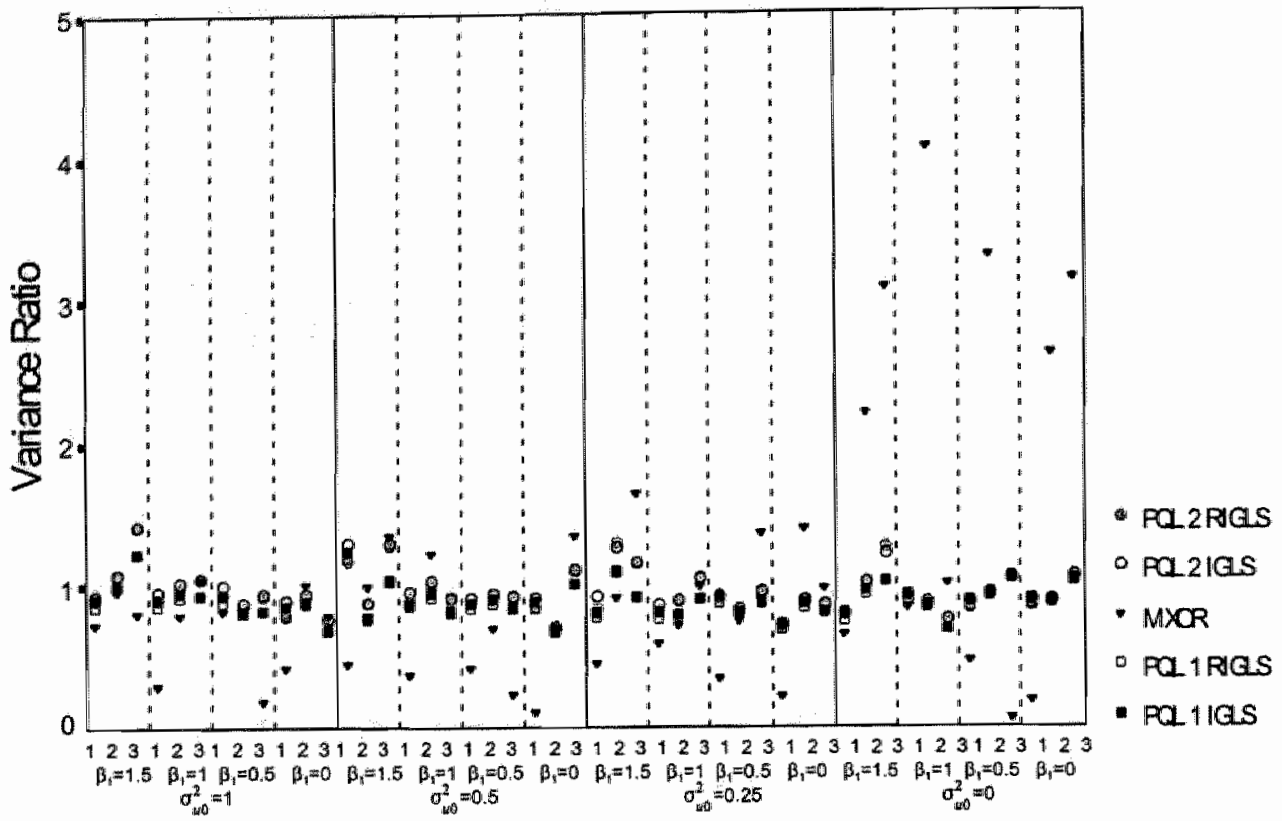

Figure 5.9. Variance Ratio $\left(\beta_{1}\right)$ as a function of the simulation conditions for a model with randomization at the person level and a random slope with true $\sigma_{w 1}^{2}=0$. Allocations of units are denoted ' $1 "=\left(n_{1}, n_{2}\right)=(40,10), 2^{\prime \prime}=\left(n_{1}, n_{2}\right)=(20,20), 3^{\prime}=\left(n_{1}, n_{2}\right)=(10,40)$.

\subsection{Discussion and conclusions}

In this study we compared first and second order PQL estimation and estimation by numerical integration for the analysis of multilevel binary data. The comparison was based on criteria concerning point estimation of parameters, and the distribution of the test statistics for testing the treatment effect. Since the estimation methods are iterative, the comparison could not be made analytically and so a simulation study was necessary. Three different allocations of units and a wide range of the probability $\pi_{i j}$ of a response $y_{i j}=1$ were covered by our simulation conditions.

It first has to be noted that estimation by numerical integration may lead to evidently incorrect estimates or no output files. In that case one may choose to fit a model with one less random effect, as we did for the models with a random intercept and fixed slope (Sections 5.5 and 5.6). In Section 5.7, in which the model with a random intercept and random slope was considered, the number of generated data sets for which estimation by numerical integration resulted in evidently incorrect estimates was very large (up to 90\%). Fitting the model with a fixed slope instead, would have resulted in a comparison between PQL for models with a 


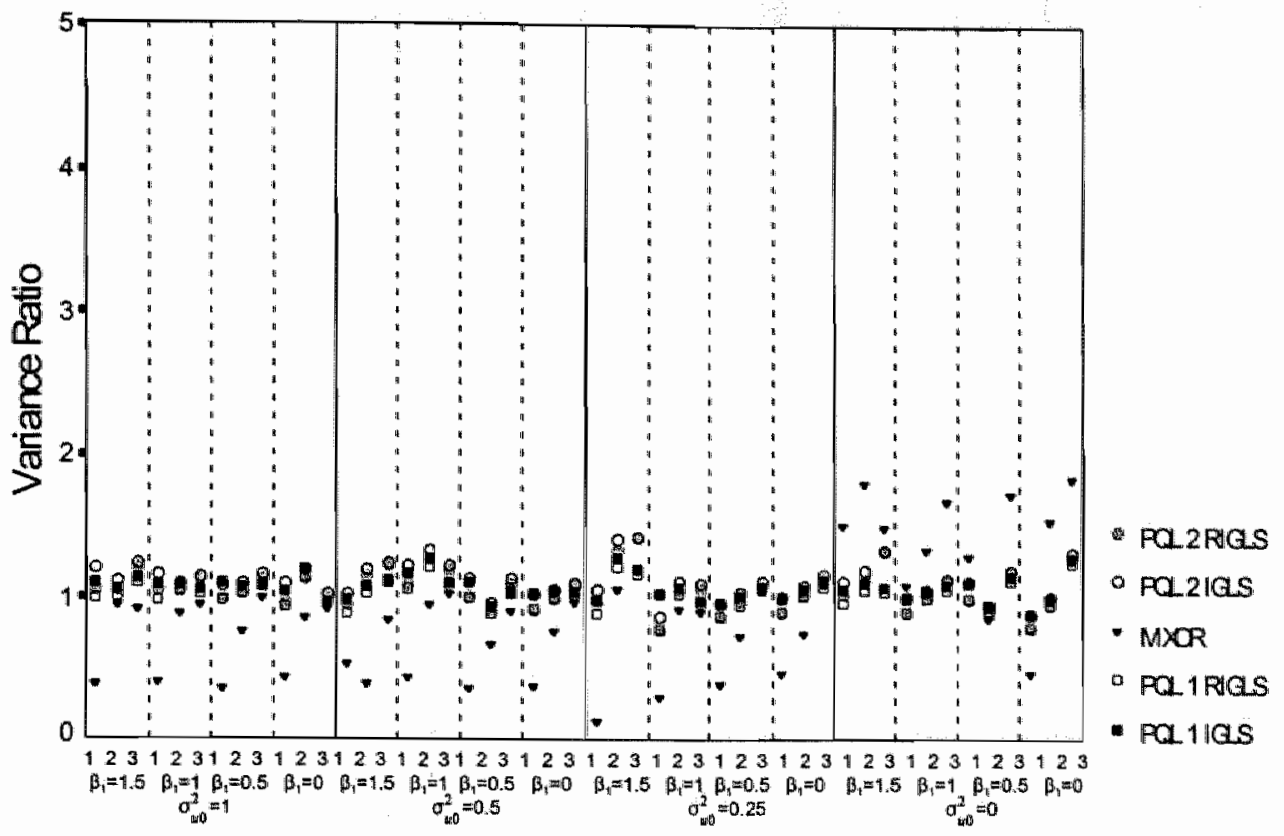

Figure 5.10. Variance Ratio $\left(\hat{\beta}_{1}\right)$ as a function of the simulation conditions for a model with randomization at the person level and a random slope with $\sigma_{u 1}^{2}=0.25$. Allocations of units are denoted ' $11^{\prime}=\left(n_{1,}, n_{2}\right)=(40,10), 2^{\prime}=\left(n_{1}, n_{2}\right)=(20,20), 3^{\prime}=\left(n_{1}, n_{2}\right)=(10,40)$.

random slope, and estimation by numerical integration for models with a fixed slope. We found this an unfair comparison and chose to consider only those data sets for which correct estimates were produced by estimation by numerical integration. Had we chosen to fit the model with a fixed slope, this would have resulted in an underestimation of the variance of the treatment effect estimator if the true $\sigma_{u 1}^{2}>0$.

Although the differences in MSE of the estimation methods were not very large, first order PQL estimation performed best in terms of point estimation of $\beta_{1}$ since it produces the smallest MSE. On the other hand, the test statistic for testing the treatment effect has not a standard normal distribution when first order PQL estimation is used, since this estimation method leads to downward biased estimates (with a relative bias of at most $10 \%$ of the true treatment effect) and consequently the mean of the distribution will shift from zero. For second order PQL estimation the treatment effect and its variance are almost unbiasedly estimated and as follows from normal probability plots the standard normal distribution may be used as reference distribution under the null hypothesis. For estimation by numerical integration this test statistic will have too long tails when $\sigma_{\mathrm{w} 0}^{2}=0$, and generally too short 
tails otherwise. For this estimation method one may choose to use test-based confidence intervals and likelihood ratio tests for fixed effects instead of the Wald test. This is not possible for $\mathrm{PQL}$ estimation since this method only calculates a quasi likelihood.

In conclusion, first order PQL has the smallest $\operatorname{MSE}\left(\beta_{1}\right)$, but due to the large bias in $\beta_{1}$ the test statistic for testing the treatment effect does not have a standard normal distribution under the null hypothesis. Second order PQL and estimation by numerical integration have a MSE just slightly larger than first order PQL. For second order PQL the standard normal distribution may be used as a reference distribution under the null hypothesis, which is not the case for estimation by numerical integration when the variance components are small. So for the cases covered by these simulation conditions we recommend second order PQL for the analysis of multilevel binary data.

\section{References}

Breslow, N. E., \& Clayton, D. G. (1993). Approximate inference in generalized linear models. Journal of the American Statistical Association, 88, 9-25.

Breslow, N. E., \& Lin, X. (1995). Bias correction in generalised linear mixed models with a single component of dispersion. Biometrika, 82, 81-91.

Bryk, A. S., Raudenbush, S. W., \& Congdon, R. T., Jr. (1996). HLM: Hierarchical Linear and Nonlinear Modeling with the HLM/2L and HLM/3L Programs. Chicago: Scientific Software International.

Gibbons, R. D., \& Hedeker, D. (1997). Random effects probit and logistic regression models for three-level data. Biometrics, 53, 1527-1537.

Goldstein, H. (1986). Multilevel mixed linear model analysis using iterative generalized least squares. Biametrika, 73, 43-56.

Goldstein, H. (1989). Restricted unbiased iterative generalized least squares estimation. Biometrika, 76, 622-623.

Goldstein, H. (1991). Nonlinear multilevel models, with an application to discrete response data. Biometrika, 78, 45-51.

Goldstein, H. (1995). Multilevel statistical models, 2nd ed. London: Edward Anold.

Goldstein, H., \& Rasbash, J. (1996). Improved approximations for multilevel models with binary responses. Journal of the Royal Statistical Society, Series A, 159, 505-513.

Goldstein, H, Rasbash, J, Plewis, I, Draper, D., Browne, W., Yang, M., Woodhouse, G., \& Healy, M. (1998). A user's guide to MLwiN, London: Institute of Education.

Hedeker, D., \& Gibbons, R. D. (1994). A random-effects ordinal regression model for multilevel analysis. Biometrics, 50, 933-944.

Hedeker, D., \& Gibbons, R. D. (1996). MIXOR: a computer program for mixed-effects ordinal regression analysis. Computer Methods and Programs in Biomedicine, 49, 157-176.

Moerbeek, M., Van Breukelen, G. J. P., \& Berger, M. P. F. (1999). Optimal experimental designs for multilevel logistic models. Manuscript submitted for publication.

Rodriguez, $G_{2}$ \& Goldman, N. (1995). An assessment of estimation procedures for multilevel models with binary data. Journal of the Royal Statistical Society Series A, 158, 73-89.

Zhou, X.-H., Perkins, A. K., \& Hui, S. L. (1999), Comparisons of software packages for generalized linear multilevel models. The American Statistician, 53, 282-290. 


\title{
Chapter 6
}

\section{Optimal experimental designs for multilevel logistic models}

\begin{abstract}
When designing experiments in multilevel populations the following three questions arise: what is the optimal level of randomization, what is the optimal allocation of units and what is the power of the test of no treatment effect? In this paper these questions will be dealt with for populations with two levels of nesting and binary outcomes. The multilevel logistic model, which is used to describe the relationship between treatment condition and outcome, is limearized. The variance of the regression coefficient associated with treatment condition in the linearized model is used to find the optimal level of randomization and the optimal allocation of units. An analytical expression for this variance can only be obtained for the first order MQL linearization method, which is known to be biased. A simulation study shows that $\mathrm{PQL}$ linearization and numerical integration of the likelihood lead to conclusions about the optimal design similar to those obtained by the analytical derivations for first order MQL.
\end{abstract}

Key words: multilevel logistic model, optimal experimental design, level of randomization, allocation of units, simulation study

Acknowledgments: We wish to thank Donald Hedeker for adjusting his MLXOR program so that the maximum number of iterations can be specified. 


\subsection{Introduction}

In many fields of science, experiments are carried out to assess the effect of different treatments on outcome variables measured at the levell of individuals. These individuals often come from multilevel populations, in which individuals are nested within clusters. For example, in the multi-center trial by Bach et al. (1995) on the effect of a new medication on the recovery from acute uncomplicated pyelonephritis, patients were nested within clinics. In the study by De Vries et al. (1994) on the effect of a smoking prevention program on smoking behavior of pupils, pupils were nested within schools. In the smoking cessation study by Hedeker, McMahon, Jason, and Salina (1994) employees were nested within worksites. Outcomes within the same cluster tend to be more alike than outcomes from different clusters. Models which take into account the nesting of individuals within clusters, and the dependency of outcomes within the same cluster, are referred to as multilevel models (Hox, 1994; Goldstein, 1995; Kreft and De Leeuw, 1998, Snijders and Bosker, 1999), hierarchical models (Bryk and Raudenbush, 1992), or random coefficient models (Longford, 1995).

Relatively little attention has so far been given to the planning of experiments in multilevel populations. Three design issues may arise when such experiments are planned. The first concerns the optimal level of randomization to treatment conditions. In principle, randomization can be done at each level of the multilevel data structure and we may wonder to what extent these differ in terms of the efficiency of the parameter estimators. The second design issue concerns the optimal allocation of units, given a fixed budget for sampling and measuring units and the level of randomization. Is it preferable to sample a few clusters with a lot of individuals per cluster, or to sample many clusters with only a few individuals per cluster? The third design issue deals with the power of the test of no treatment effect, i.e. the probability of rejecting the null hypothesis of no treatment effect when this hypothesis is not true.

For experiments in multilevel populations with continuous responses, optimal sample sizes have been studied analytically by Donner, Birkett and Buck (1981), Hsieh (1988), Shih (1997), Liu and Liang (1997), Snijders and Bosker (1993), Raudenbush (1997), and Moerbeek, Van Breukelen, and Berger (1999, in press). For binary responses research has been restricted to the test of the difference between two proportions, which is not easily extended with covariates. For cluster randomized studies, optimal sample sizes were presented by Donner, Birkett, and Buck(1981), Hsieh (1988), Feng and Grizzle (1992), Lee and Dubin (1994), Liu and Liang (1997), Shih (1997), and Donner (1998) under the condition that either the number of clusters or the number of individuals per cluster is fixed.

The remainder of this paper is as follows. In Section 6.2 the multilevel logistic model is introduced to describe the relationship between treatment condition and outcome. In this section two common estimation procedures for the multilevel logistic model will be discussed. The first is based on linearization of the model and appears in two different forms: Marginal Quasi Likelihood (MQL; Goldstein, 1991; Breslow and Clayton, 1993), and 
Penalized Quasi Likelihood (PQL; Goldstein and Rasbash, 1996; Breslow and Clayton, 1993). The second estimation procedure is based on numerical integration of the marginal likelihood (Hedeker and Gibbons, 1994; Gibbons and Hedeker, 1997).

The establishment of the optimal level of randomization and the optimal allocation of units for each level of randomization will be based on the efficiency of the estimator of the treatment effect. The multilevel logistic model is a non-linear model, and an analytical expression for the variance of this estimator can only be derived by linearization and using first order MQL. This method is known to produce downwardly biased parameter estimates (Rodriguez and Goldman, 1995; Goldstein and Rasbash, 1996), while PQL and numerical integration perform better. As optimal designs cannot be derived analytically for $\mathrm{PQL}$ and numerical integration, simulations will be reported to show how the variance of the treatment effect estimator is affected by using PQL or numerical integration instead of first order MQL. In this way, optimal experimental designs are given for models without and with random slopes in Sections 6.3 and 6.4 , respectively. In Section 6.5 , the power of the test of no treatment effect will be discussed. Finally, conclusions and suggestions for further research will be given in Section 6.6. In this section some attention will also be paid to optimal designs based on practical or ethical criteria, which may or may not be compatible with optimal designs based on statistical criteria.

\subsection{The multilevel logistic model and its estimation using Quasi Likelihood or numerical integration}

In this section the multilevel logistic model with a random intercept and a fixed slope is given. The generalization to a model with a random slope is straightforward and will be discussed in Section 6.4 As an example, a smoking prevention intervention, with pupills nested within schools, is used. Pupils and schools are the units at level one and two, respectively. The response variable smoking behavior is denoted by $y_{i j}$ for pupil $i$ within. school $j$, and measured on a binary scale, i.e. a pupil smokes or does not after the interwention has taken place. Treatment condition will be denoted by $x_{i j}$ with values -1 and +1 for the control and intervention group, respectively.

The optimal designs that will be derived are balanced, i.e. we have a random sample of $j=1, \ldots, n_{2}$ schools and $i=1, \ldots, n_{1}$ pupils per school. In principle, randomization to treatment conditions can be done at any level. If randomization is done at the pupil level, then $\frac{1}{2} n_{1}$ pupils within each school are randomized to the treatment group and $\frac{1}{2} n_{1}$ are randomized to the control group, and thus $n_{1}$ has to be even. Similarly, if randomization is done at the school level, $\frac{1}{2} n_{2}$, schools are randomized to the control group and $\frac{1}{2} n_{2}$ schools are randomized to the intervention group, and thus $n_{2}$ has to be even.

We will assume that, conditional on all fixed and random effects in the model, the responses $y_{i j}$ are independently and Bernoulli distributed with probabilities $\pi_{i j}$ " 
$y_{i j} \sim$ Bemoulli $\left(1, \pi_{i j}\right)$. These probabilities are related to the treatment condition $x_{i j}$ by the logit link function

$$
\operatorname{logit} \pi_{i j}=\ln \left(\frac{\pi_{i j}}{1-\pi_{i j}}\right)=\beta_{0}+\beta_{1} x_{i j}+u_{0, j^{*}}
$$

where logit $\pi_{i j}$ is the linear predictor consisting of a fixed part $\left(\beta_{0}+\beta_{1} x_{i j}\right)$ and a random part $u_{0 j}$. The parameter $u_{0 j}$ represents the random departure for school $j$ with $u_{0 j}-\mathrm{N}\left(0, \sigma_{u j}^{2}\right)$. This random term is assumed to be independently distributed and independent of the treatment condition.

Equation (6.1) can be rewritten as

$$
\pi_{i j}=f\left(\beta_{0}+\beta_{1} x_{i j}+u_{0 j}\right)=\left[1+\exp \left\{-\left(\beta_{0}+\beta_{1} x_{i j}+u_{0 j}\right)\right\}\right]^{-1} .
$$

The responses can now be written as the probability $\pi_{i j}$ plus a level one residual denoted by $e_{i j}$ :

$$
y_{i j}=\pi_{i j}+e_{i j}
$$

where $e_{i j}$ has zero mean and variance $\pi_{i j}\left(1-\pi_{i j}\right)$ since var $\left(e_{i j}\right)=\operatorname{var}\left(y_{i j}\right)$ and $y_{i j} \sim \operatorname{Bernoulli}\left(1, \pi_{i j}\right)$.

The likelihood function, conditional upon the fixed and random effects has the form:

$$
L\left(y \mid \beta, u_{0 j}\right)=\prod_{i j}\left(\pi_{i j}\right)^{y_{i j}}\left(1-\pi_{i j}\right)^{1-y_{i t}},
$$

where $y$ and $\beta$ are vectors containing the responses and the fixed effects, respectively. To obtain the unconditional (marginal) likelihood, we need to multiply the conditional likelihood by the density of $u_{0 j}$ and to integrate out the random effect:

$$
L\left(y \mid \beta, \sigma_{u 0}^{2}\right)=\int L\left(y \mid \beta, u_{0, j}\right) f\left(u_{0 j}\right) d u_{0 j ;}
$$

where $f\left(u_{0, j}\right)$ is the density function of $u_{0 j}$. This unconditional likelihood is intractable, but various approximations have been proposed of which two will be discussed in this section. The first is to approximate the marginal likelihood function by numerical integration and has been used by Hedeker and Gibbons (1994) and Gibbons and Hedeker (1997). For two levels of nesting this method is implemented in the computer program MLXOR (Hedeker and Gibbons, 1996). The second method is to linearize the nonlinear part using a Taylor series expansion around the current estimated regression coefficients $\bar{\beta}$ and around the current estimated residual (posterior mean) $\hat{u}_{0 j}$ (Goldstein and Rasbash, 1996):

$$
\pi_{i j}=f\left(X_{i j} \beta+u_{0 j}\right)=f(H)+X_{i j}(\beta-\beta) f^{\prime}(H)+\left(u_{0 j}-\hat{u}_{0 j}\right) f^{\prime}(H)+\left(u_{0 j}-\hat{u}_{0 j}\right)^{2} f^{\prime \prime}(H) / 2,
$$

in which $H=X_{i j} \beta+\hat{u}_{0 j}$. This method has been referred to as Penalized Quasi-Likelihood (PQL) 
by Breslow and Clayton (1993). Omitting the last term in (6.6) yields a first order approximation. Linearization can also be done around the fixed part of the linear predictor only, and then $\hat{u}_{0 j}$ in $H$ and equation (6.6) is set equal to zero (Goldstein, 1991). This method has been referred to as Marginal Quasi-Likelihood (MQL) by Breslow and Clayton (1993), and again a first order approximation is possible. The linearized model can be estimated using Iterative Generalized Least Squares (IGLS, Goldstein, 1986) or Restricted Iterative Generalized Least Squares (RIGLS, Goldstein, 1989). Both MQL and PQL are implemented in MLwiN (Goldstein, et al. 1998). First order PQL is implemented in the program HLM as well (Bryk, Raudenbush and Congdon, 1996).

\subsection{Optimal experimental designs for the model with random intercept and}

\section{fixed slope}

\subsubsection{Optimal experimental designs for first order MQL with known variance components}

The optimal level of randomization and allocation of units are chosen such that $\operatorname{Var}\left(\hat{\beta}_{1}\right)$ is minimized. An analytical expression for $\operatorname{Var}\left(\hat{\beta}_{1}\right)$ using first order MQL with known variance components can be derived as follows. Following Goldstein and Rasbash (1996), (6.6) with $\hat{u}_{0 j}=0$ and omitting the second order term is substituted into (6.3). Rearranging slightly and dividing by $f^{\prime}(H)=\tilde{\pi}_{i j}\left(1-\tilde{\pi}_{i j}\right)$, where $\tilde{\pi}_{i j}=f\left(X_{i j} \beta\right)$, gives

$$
y_{i j}^{*}=\boldsymbol{X}_{i j} \beta+u_{0 j}+e_{i j}^{*} \text {, }
$$

where the transformed response is

$$
y_{i j}^{*}=X_{i j} \beta+\left[\tilde{\pi}_{i j}\left(1-\tilde{\pi}_{i j}\right)\right]^{-1}\left(y_{i j}-\tilde{\pi}_{i j}\right),
$$

and the transformed error term is $e_{i j}^{*}=\left[\tilde{\pi}_{i j}\left(1-\tilde{t}_{i j}\right)\right]^{n} e_{i j}$. If $\operatorname{Var}\left(e_{i j}{ }^{*}\right)$ is approximated by $\left[\tilde{\pi}_{i j}\left(1-\tilde{\pi}_{i j}\right)\right]^{-1}$, we obtain

$$
\operatorname{Var}\left(y_{i j}^{*}\right) \approx \sigma_{u 0}^{2}+\left[\tilde{\pi}_{i j}\left(1-\tilde{\pi}_{i j}\right)\right]^{-1} .
$$

Once $\beta$ is specified or known from a preceding iteration, the linearized model in (6.7) can be estimated by standard estimating procedures from the linear multilevel model.

For the derivation of $\operatorname{Var}\left(\hat{\beta}_{1}\right)$ we assume that the variance component $\sigma_{w 0}^{2}$ is known. In this case, $\beta_{1}$ can be estimated by the generalized least squares estimator. Assuming that $x_{i j}$ is coded by -1 and $+1, \hat{\beta}_{1}$ will become

$$
\hat{\beta}_{1}=\frac{\bar{y}_{. t}^{*}-\bar{y}_{. c}^{*}}{2}
$$


where $\vec{y}^{*}$ and $\vec{y}^{*}{ }_{i,}$ are the mean transformed responses in the treatment and control group, respectively. An approximation for the expectation of $\beta_{1}$ using first order MQL can be calculated by using the normal approximation of the logistic function, which results in

$$
\begin{array}{r}
\mathrm{E}\left(\hat{\beta}_{1}\right)=\beta_{1}+\frac{1}{2}\left\{\left[1+\exp \left(\frac{-\beta_{0}-\beta_{1}}{c}\right)\right]^{-1}-\left[1+\exp \left(-\beta_{0}-\beta_{1}\right)\right]^{-1}\right\}\left[2+\exp \left(-\beta_{0}-\beta_{1}\right)+\exp \left(\beta_{0}+\beta_{1}\right)\right] \\
-\frac{1}{2}\left\{\left[1+\exp \left(\frac{-\beta_{0}+\beta_{1}}{c}\right)\right]^{1-1}-\left[1+\exp \left(-\beta_{0}+\beta_{1}\right)\right]^{-1}\right\}\left[2+\exp \left(-\beta_{0}+\beta_{1}\right)+\exp \left(\beta_{0}-\beta_{1}\right)\right],
\end{array}
$$

where $c=\sqrt{1+\sigma_{t 0}^{2} / 1.7^{2}}$. From this formula it can be concluded that if the design is balanced and first order MQL is used, $\mathrm{E}\left(\hat{\beta}_{1}\right)$ depends on $\sigma_{w 0}^{2}$ (if $\sigma_{w 0}^{2}$ is large, the bias is also large) and the regression coefficients, but not on the level of randomization or on the allocation of units.

The corresponding $\operatorname{Var}\left(\hat{\beta}_{1}\right)$ obtained with the generalized least squares estimator is given in the second column of Table 6.1 (see the appendix for the derivation), and is equal to that for the case of continuously distributed outcomes given by Moerbeek et al. (in press), except that the variance component at the pupil level in the linear case, $\sigma_{e}^{2}$, is now replaced by $\delta^{2}=\frac{1}{2}\left(4+e^{\beta_{0}+\beta_{1}}+e^{\beta_{0}-\beta_{1}}+e^{-\beta_{0}+\beta_{1}}+e^{-\beta_{0}-\beta_{1}}\right)$, and the same replacement holds for the optimal allocation of units and $\operatorname{Var}\left(\hat{\beta}_{1}\right)$ given the optimal allocation of units that will presented in this section. Furthermore this replacement may also be applied to the results for three levels of nesting as given by Moerbeek et al. (in press). Note that $\delta^{2} \geq 4 \forall \beta_{0}, \beta_{1}$. As the treatment conditions are coded -1 and $+1,2 \beta_{1}$ is equal to the log-odds ratio and $4 \delta^{2}$ is approximately equal to the expectation of the variance estimator of the log-odds ratio as proposed by Woolf (1955) for logistic models without random effects.

Table 6.l $\operatorname{Var}\left(\hat{\beta}_{1}\right)$, Optimal Allocation of Units, and $\operatorname{Var}\left(\hat{\beta}_{1}\right)$ given Optimal Allocation of Units First Order MQL with Known Variance Components Models with Random Intercepts and Fixed Slopes

\begin{tabular}{lllll}
\hline $\begin{array}{l}\text { Level of } \\
\text { randomization }\end{array}$ & $\operatorname{Var}\left(\hat{\beta}_{1}\right)$ & optimal $n_{1}$ & optimal $n_{2}$ & $\operatorname{Var}\left(\hat{\beta}_{1}\right)$ given optimal $n_{1}, n_{2}$ \\
\hline pupil & $\frac{\delta^{2}}{n_{1} n_{2}}$ & $\frac{C-2 c_{2}}{2 c_{1}}$ & 2 & $\frac{\delta^{2} c_{1}}{C-2 c_{2}}$ \\
school & $\frac{n_{1} \sigma_{u 0}^{2}+\delta^{2}}{n_{1} n_{2}}$ & $\frac{\delta}{\sigma_{u 0}} \sqrt{\frac{c_{2}}{c_{1}}}$ & $\frac{C}{\frac{\delta}{\sigma_{u 0}} \sqrt{c_{1} c_{2}}+c_{2}}$ & $\frac{\left(\sigma_{u 0} \sqrt{c_{2}}+\delta \sqrt{c_{1}}\right)^{2}}{C}$ \\
\end{tabular}

Note. Treatment conditions are coded -1 and +1 .

$\delta^{2}=\frac{1}{2}\left(4+e^{\beta_{0}+\beta_{1}}+e^{\beta_{0}-\beta_{1}}+e^{-\beta_{0}+\beta_{1}}+e^{-\beta_{0}-\beta_{1}}\right)$. 


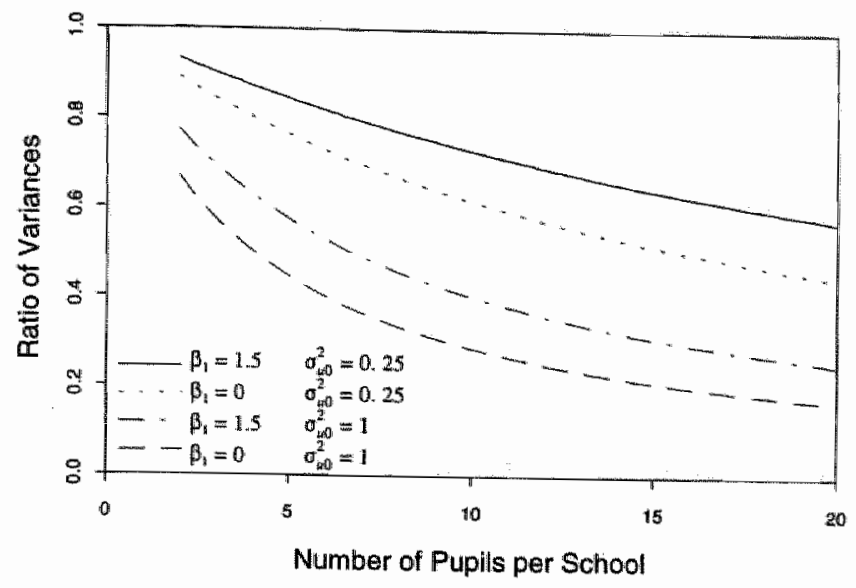

Figure 6.1. Ratio of the $\operatorname{Var}\left(\hat{\beta}_{1}\right)$ for randomization at the pupil level and the $\operatorname{Var}\left(\hat{\beta}_{1}\right)$ for randomization at the school level and first order MQL with known variance components.

From Table 6.1 it is clear that the pupil level is the optimal level of randomization because $\operatorname{Var}\left(\hat{\beta}_{1}\right)$ is the smallest for this level of randomization. Randomization at higher levels will lead to higher values for $\operatorname{Var}\left(\hat{\beta}_{1}\right)$. This is shown in Figure 6.1 , in which the ratio of the variances for randomization at the pupil level and randomization at the school level is plotted as a function of $n_{1}$ and for parameter values $\left(\beta_{0}, \beta_{1}, \sigma_{u 0}^{2}\right)=(0,0,1),(0,1.5,1)$, $(0,0,0.25)$, and $(0,1.5,0.25)$. Figure 6.1 shows that the ratio of the variances moves away from 1 when $n_{1}$ and $\sigma_{u 0}^{2}$ increase, and tends towards 1 when $\beta_{1}$ increases. From Figure 6.1 it is clear that for the displayed parameter values and $n_{1}$ randomization at the school level may become very inefficient when compared with randomization at the pupil level, at least when a fixed slope is assumed.

Since randomization at the pupil level may not always be feasible, the optimal allocation of units is derived for both levels of randomization. Optimal allocations will be derived under the condition that the total costs for sampling and measuring units do not exceed the budget for sampling and measuring units which is fixed to $C$. The costs for sampling and measuring pupils and schools are denoted $c_{1}, c_{2}$, respectively. When these costs differ across treatment conditions, the mean costs should be taken. Then we have the following optimization problem: 


$$
\text { minimize } \operatorname{Var}\left(\hat{\beta}_{1}\right)_{s}
$$

subject to the condition

$$
C=c_{1} n_{1} n_{2}+c_{2} n_{2},\left(c_{l}>0, n_{l} \geq 2 \text { for } l=1,2\right) .
$$

We assume $n_{1}$ and $n_{2}$ to be at least two in order to maintain a multilevel data structure and to be able to estimate the variance component $\sigma_{u 0}^{2}$. The optimal allocation of units can be obtained by expressing $n_{1}$ in terms of $n_{2}$ and the costs using (6.13), substituting this formula into the formula for $\operatorname{Var}\left(\beta_{1}\right)$ as given in the second column of Table 6.1, and solving for $n_{2}$. The obtained optimal allocations and the minimum $\operatorname{Var}\left(\hat{\beta}_{1}\right)$ for these optimal allocations are in columns three and four, and the fifth column of Table 6.1 , respectively. $A$ lower $\operatorname{Var}\left(\beta_{1}\right)$ can be obtained by using a higher budget $C$, which results in sampling more units only at the level at which randomization is done since the sample size at the other level is independent of C. Note that the optimal allocation of units for randomization at the pupil level and assuming no treatment by school interaction does not depend on the parameter values and is therefore globally optimal. For randomization at the school level, however, the design is locally optimal and the parameter values must be known or a reasonable guess must be available before the optimal allocation of units can be found. For pupil level randomization the optimal number of schools is equal to two. With such a small number of schools the school level variance component cannot be estimated well, but the variance of the treatment effect estimator does not depend on this parameter. Also just one school is needed to estimate the treatment effect if there is no treatment by school interaction. However, in general one might wonder whether such a small number of schools in a random sample may give enough information about their population so that the multilevel model may be used for the data analysis. The fixed effects model, in which the school effects are included using dummy variables, may perhaps be a more appropriate model for such a small number of schools, and a rule of thumb for choosing between the fixed effects model and the multilevel model is given in Snijders and Bosker (1999, p. 44).

\subsubsection{Optimal experimental designs for $\mathrm{PQL}$ and numerical integration}

In this section the results on optimal designs for first order MQL with known variance components are translated into results for PQL and numerical integration with unknown variance components. Four different $\mathrm{PQL}$ methods are available in the computer program MLwiN (Goldstein et al., 1998): first order PQL with IGLS or RIGLS, and second order PQL with IGLS or RIGLS. The maximum number of iterations was set equal to 100 , the convergence criterion was set equal to 0.001 for the relative change in parameter estimates. The computer program MIXOR (Hedeker and Gibbons, 1996) was used for estimation by 
means of numerical integration. We used ten quadrature points as was suggested by Hedaker and Gibbons (1996), and automatic starting values for the iterative estimation process for which the maximum number of iterations was set to 1000 , and a convergence criterion of 0.001 for the absolute change in parameter estimates. Especially for small variance $\sigma_{v 0}^{2}$ at the school level, estimation by numerica integration may yield an unrealistic high estimate $\partial_{u 0}^{2}$. For those cases for which $\hat{\sigma}_{w 0}^{2}>3$, the estimation of the model with a fixed intercept were used instead since fitting a model with a fixed slope is the most logical thing to do in practice, and sometimes this is automatically done by MIXOR as well. This mainly occurred for randomization at the school level, where the true $\sigma_{u f 0}^{2}$ was zero, and the number of schools was small $\left(n_{2}=10\right)$.

Data sets for the multilevel logistic model given by $(6.2)$ and $(6.3)$ were simulated. Three different allocations were considered, namely $\left(n_{1}=40, n_{2}=10\right),\left(n_{1}=20, n_{2}=20\right)$, and $\left(n_{1}=10, n_{2}=40\right)$. The parameter values were $\beta_{0}=0 ; \beta_{1}=1.5,1,0.5$, or $0 ; 0_{w 0}^{2}=1,0.5,0.25$, or 0 . Thus, there are 96 simulation combinations of level of randomization, allocation of units, and parameter values. For each of the 96 combinations, 200 data sets were generated.

Given $\beta_{1}>0$ and $x_{i j}$ coded as -1 and +1 , the range of the probability $\pi_{i j}$ of a positive response $\left(y_{i j}=1\right)$ is approximately:

$$
\text { range } \pi_{i j}=\left(\left[1+\exp \left(-\beta_{0}+\beta_{1}+2 \sigma_{u 0}\right)\right]^{-1},\left[1+\exp \left(-\beta_{0}-\beta_{1}-2 \sigma_{u i 0}\right)\right]^{-1}\right) \text {. }
$$

For the given parameter values the range of $\pi_{i j}$ is given in Table 6.2 , showing that a wide range for the probability $\pi_{i j}$ is covered by our simulation conditions.

Table 6.2 Ranges of the Probability $\pi_{\mathrm{ij}}$ for the Parameter Values

\begin{tabular}{lllll}
\multicolumn{5}{c}{ Models with Random Intercepts and Fixed Slopes } \\
\cline { 2 - 5 }$\sigma_{\text {*10 }}^{2}$ & 1.5 & 1 & \multicolumn{5}{c}{0.5} & 0 \\
\hline 1 & $0.03-0.97$ & $0.05-0.95$ & $0.08-0.92$ & $0.12-0.88$ \\
0.5 & $0.05-0.95$ & $0.08-0.92$ & $0.12-0.88$ & $0.20-0.80$ \\
0.25 & $0.08-0.92$ & $0.12-0.88$ & $0.18-0.82$ & $0.27-0.73$ \\
0 & $0.18-0.82$ & $0.27-0.73$ & $0.38-0.62$ & $0.50-0.50$ \\
\hline
\end{tabular}

The five estimation procedures were used for the simulated data, and for each of the 96 simulation combinations the Sampling Variance was estimated, where

$$
\text { Sampling Variance }\left(\hat{\beta}_{1}\right)=\left(\sum_{r=1}^{200}\left(\hat{\beta}_{1, r}-\sum_{r=1}^{200} \hat{\beta}_{1 / r} / 200\right)^{2}\right) / 199 \text {, }
$$


and $\beta_{1 r}$ is the estimate of $\beta_{1}$ from the $r$ th simulated data set. Furthermore, a Correction Factor is calculated, which is the ratio of the Sampling Variance $\left(\hat{\beta}_{1}\right)$ and the $\operatorname{Var}\left(\hat{\beta}_{1}\right)$ obtained from first order MQL with known variance components. This Correction Factor can be used as a correction for the $\operatorname{Var}\left(\beta_{1}\right)$ in Table 6.1 when $\mathrm{PQL}$ or numerical integration is used instead of first order MQL with known variance components. In Table 6.3 the mean values of the Sampling Variance $\left(\hat{\beta}_{1}\right)$ and Correction Factor are given, averaged across the levels of the other designs factors. Consider, for example, the 16 simulation combinations for which randomization is done at the pupil level and $n_{1}=10, n_{2}=40$. The reported results are the average across 16 simulation combinations for varying $\beta_{1}\left(\beta_{1}=0,0.5,1,1.5\right)$ and $\sigma_{u 0}^{2}$ $\left(\sigma_{u 0}^{2}=0,0.25,0.5,1\right)$. So Sampling Variance $\left(\beta_{1}\right)=0.013$ for first order $P Q L$ and is the mean Sampling Variance $\left(\beta_{1}\right)$ of those 16 simulation combinations for which randomization is done at the pupil level and $n_{1}=10, n_{2}=40$.

From Table 6.3 it follows that for randomization at the school level the Sampling Variance $\left(\beta_{1}\right)$ increases with $\beta_{1}, \sigma_{y 0}^{2}$, and the number of pupils per school, given the total sample size and given that $\beta_{0}=0$. For randomization at the pupil level the Sampling Variance $\left(\beta_{1}\right)$ only increases with $\beta_{1}$, given $\beta_{0}=0$. Furthermore, the Sampling Variance $\left(\beta_{1}\right)$ is larger for randomization at the school level, especially when $\sigma_{u 0}^{2}$ and/or the number of pupils per school is large. These conclusions apply to first order MQL with known variance components as well, see Table 6.1. The $\operatorname{Var}\left(\hat{\beta}_{1}\right)$ obtained with first order MQL with known variance components has to be multiplied by the Correction Factor, which is about 1.0 for first order $\mathrm{PQL}$, and about 1.2 for second order $\mathrm{PQL}$ and numerical integration. The Correction Factor is almost constant across the allocations of units. So, the Correction Factor for the optimal sample sizes obtained with the analytical formulae for first order MQL with known variance components will be almost equal to that for the non-optimal sample sizes. Therefore, the optimal sample sizes obtained with first order MQL with known variance components will generally also be optimal for estimation by $\mathrm{PQL}$ or numerical integration. of course, the $\operatorname{Var}\left(\beta_{1}\right)$ obtained with PQL or estimation by numerical integration will be larger than that for first order MQL since the Correction Factor is larger than one.

The simulation study further showed that $\beta_{1}$ was underestimated by at most $10 \%$ of the true $\beta_{1}$ by first order $\mathrm{PQL}$ and overestimated by at most $5 \%$ of the true $\beta_{1}$ by second order $\mathrm{PQL}$ and numerical integration. Generally the smallest bias is obtained by the latter method. Furthermore the ratio of the Sampling Variance $\left(\beta_{1}\right)$ and the Mean Variance $\left(\beta_{1}\right)$, which is the mean of the $200 \operatorname{Var}\left(\beta_{1}\right)$ as reported by the software, was calculated for each of the 96 simulation combinations. This ratio measures the unbiasedness of the reported $\operatorname{Var}\left(\hat{\beta}_{1}\right)$ and should be close to unity, as was the case for first and second order PQL. For numerical integration, however, this ratio may be as large as 2 for $\sigma_{z 0}^{2}=0$, and between 0.5 and 1.5 for $\sigma_{u t}^{2}>0$. 
Table 6.3 Sampling Variance $\left(\hat{\beta}_{1}\right)$ (Correction Factor) for PQL and Numerical Integration Models with Random Intercepts and Fixed Slopes

\begin{tabular}{|c|c|c|c|c|c|}
\hline $\begin{array}{l}\text { Parameter/ } \\
\text { Allocation }\end{array}$ & $\begin{array}{l}\text { PQL-1 } \\
\text { IGLS } \\
\end{array}$ & $\begin{array}{l}\text { PQL-2 } \\
\text { IGLS }\end{array}$ & $\begin{array}{l}\text { PQL-1 } \\
\text { RIGLS }\end{array}$ & $\begin{array}{l}\text { PQL-2 } \\
\text { RIGLS }\end{array}$ & $\begin{array}{l}\text { Numerical } \\
\text { integration }\end{array}$ \\
\hline \multicolumn{6}{|c|}{ Randomization at school level } \\
\hline$\sigma_{100}$ & $0.071(0.975)$ & $0.081(1.140)$ & $0.072(0.997)$ & $0.085(1.188)$ & $0.107(1.399)$ \\
\hline 0.5 & $0.041(0.959)$ & $0.045(1.077)$ & $0.041(0.975)$ & $0.047(1.113)$ & $0.051(1.167)$ \\
\hline 0.25 & $0.026(0.981)$ & $0.029(1.063)$ & $0.027(0.992)$ & $0.029(1.091)$ & $0.028(1.058)$ \\
\hline 0 & $0.013(1.022)$ & $0.013(1.072)$ & $0.013(1.023)$ & $0.013(1.059)$ & $0.014(1.095)$ \\
\hline \multicolumn{6}{|l|}{$\beta_{1}$} \\
\hline 1.5 & $0.043(0.984)$ & $0.049(1.125)$ & $0.044(1.000)$ & $0.052(1.174)$ & $0.061(1.259)$ \\
\hline 1 & $0.039(0.994)$ & $0.043(1.091)$ & $0.039(1.007)$ & $0.045(1.123)$ & $0.051(1.183)$ \\
\hline 0.5 & $0.035(0.986)$ & $0.039(1.096)$ & $0.036(0.998)$ & $0.040(1.094)$ & $0.045(1.154)$ \\
\hline 0 & $0.034(0.972)$ & $0.037(1.040)$ & $0.034(0.982)$ & $0.038(1.061)$ & $0.042(1.123)$ \\
\hline \multicolumn{6}{|l|}{$n_{1}, n_{2}$} \\
\hline 40,10 & $0.058(1.016)$ & $0.063(1.104)$ & $0.059(1.033)$ & $0.066(1.123)$ & $0.083(1.298)$ \\
\hline 20,20 & $0.033(0.989)$ & $0.037(1.077)$ & $0.034(1.002)$ & $0.038(1.106)$ & $0.040(1.137)$ \\
\hline 10,40 & $0.022(0.947)$ & $0.026(1.083)$ & $0.022(0.956)$ & $0.026(1.110)$ & $0.027(1.104)$ \\
\hline \multicolumn{6}{|c|}{ Randomization at pupil level } \\
\hline$\sigma_{u 0}^{2}$ & $0.013(1.068)$ & $0.016(1.248)$ & $0.014(1.082)$ & $0.016(1.272)$ & $0.016(1.233)$ \\
\hline 0.5 & $0.014(1.082)$ & $0.015(1.221)$ & $0.014(1.093)$ & $0.016(1.239)$ & $0.015(1.209)$ \\
\hline 0.25 & $0.013(1.063)$ & $0.015(1.171)$ & $0.013(1.071)$ & $0.015(1.187)$ & $0.015(1.164)$ \\
\hline 0 & $0.013(1.033)$ & $0.014(1.067)$ & $0.013(1.035)$ & $0.014(1.075)$ & $0.015(1.163)$ \\
\hline \multicolumn{6}{|c|}{$00100070)$} \\
\hline 1.5 & $0.018(1.076)$ & $0.021(1.258)$ & $0.018(1.089)$ & $0.022(1.286)$ & $0.021(1.266)$ \\
\hline 1 & $0.014(1.061)$ & $0.015(1.181)$ & $0.014(1.070)$ & $0.015(1.200)$ & $0.016(1.239)$ \\
\hline 0.5 & $0.011(1.046)$ & $0.012(1.133)$ & $0.011(1.053)$ & $0.012(1.145)$ & $0.012(1.129)$ \\
\hline 0 & $0.011(1.063)$ & $0.011(1.134)$ & $0.011(1.068)$ & $0.011(1.144)$ & $0.011(1.136)$ \\
\hline \multicolumn{6}{|c|}{ 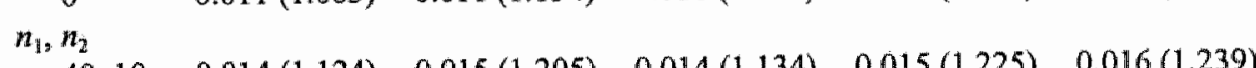 } \\
\hline 40,10 & $0.014(1.124)$ & $0.015(1.205)$ & $0.014(1.134)$ & $0.015(1.225)$ & $0.016(1.239)$ \\
\hline 20,20 & $0.013(1.034)$ & $0.014(1.141)$ & $0.013(1.043)$ & $0.015(1.156)$ & $0.014(1.142)$ \\
\hline 10,40 & $0.013(1.028)$ & $0.015(1.184)$ & $0.013(1.034)$ & $0.015(1.199)$ & $0.015(1.1 .96)$ \\
\hline
\end{tabular}




\subsubsection{An examplle: Planning of a school-based smoking prevention intervention}

We will use an example to illustrate the results from Sections 6.3 .1 and 6.3 .2 , and to illustrate possible discrepancies between optimal designs using statistical and practical criteria. Consider as an example a school-based smoking prevention intervention with pupils nested within schools in which a newly developed smoking prevention intervention will be compared to an old one. Suppose that the budget of this study is, say, $C=\$ 10000.00$, and the costs for measuring and sampling at the pupil and school level are $c_{1}=\$ 50.00$ and $c_{2}=\$ 1000.00$, respectively. Then the design can be optimized as follows.

Table 6.4 Example: Planning of a School-based Smoking Prevention Intervention

\begin{tabular}{lllllll}
\hline $\begin{array}{l}\text { Level of } \\
\text { randomization }\end{array}$ & $\begin{array}{l}\text { Constraint on } \\
\text { sample sizes }\end{array}$ & $\begin{array}{l}\text { Random } \\
\text { slope }\end{array}$ & $\begin{array}{l}\text { Optimal } \\
n_{1}\end{array}$ & $\begin{array}{l}\text { Optimal } \\
n_{2}\end{array}$ & $\begin{array}{l}\operatorname{Var}\left(\beta_{1}\right) \\
\text { PQL 2 }\end{array}$ & $\begin{array}{l}\text { Power } \\
(\Delta=0.5, \\
\alpha=0.05)\end{array}$ \\
\hline pupil & $n_{1} \geq 2, n_{2} \geq 2$ & no & 80 & 2 & 0.032 & 0.87 \\
pupil & $2 \leq n_{1} \leq 30, n_{2} \geq 2$ & no & 30 & 4 & 0.043 & 0.78 \\
school & $n_{1} \geq 2, n_{2} \geq 2$ & no & 13 & 6 & 0.115 & 0.43 \\
pupil & $n_{1} \geq 2, n_{2} \geq 2$ & yes & 30 & 4 & 0.072 & 0.59 \\
\hline
\end{tabular}

Randomization at pupil level. The optimal allacation of units for randomization at the pupil level for first order MQL with known variance components is $n_{1}=80, n_{2}=2$ (see Table 6.4). To calculate the $\operatorname{Var}\left(\hat{\beta}_{1}\right)$ for such an allocation of units, the researcher needs a reasonable estimate of the parameter values. Lets assume that there is a fixed treatment effect, and that the parameter values are $\beta_{0}=0, \beta_{1}=0.5, \sigma_{u 0}^{2}=0.25$, respectively. Then $\delta^{2}=4.255$ and $\operatorname{Var}\left(\beta_{1}\right)$ will be 0.0266 . Suppose now that the intervention aims at twelve year old pupils and that at most 30 of them can be sampled from each school. Then $n_{1}=30$ and $n_{2}=4$, and $\operatorname{Var}\left(\beta_{1}\right)$ increases to 0.0355 . For second order $P Q L$ the $\operatorname{Var}\left(\beta_{1}\right)$ will be somewhat higher as it has to be multiplied by a Correction Factor equal to 1.2. It should be noted that the variance component $\sigma_{u 0}^{2}$ cannot be estimated with minimum variance for these small values of $n_{2}$

Randomization at school level. The optimal allocation of units for randomization at the school level is $m_{1}=18.45, n_{2}=5.2$ for first order MQL with known variance components (see Table 6.1). As the number of schools has to be even, we round off to $n_{2}=6$ which enables us to sample $n_{1}=13$ pupils per school and leads to $\operatorname{Var}\left(\hat{\beta}_{1}\right)=0.0962$ (see Table 6.4). So $\operatorname{Var}\left(\beta_{1}\right)$ for randomization at the school level is almost three times as large as $\operatorname{Var}\left(\hat{\beta}_{1}\right)$ obtained with randomization at the pupil level. To obtain the same $\operatorname{Var}\left(\beta_{1}\right)$ as for pupil level randomization a budget of $\$ 26600.00$ would be needed and would result in optimal sample sizes $n_{1}=18, n_{2}=14$. Again, multiplication by the Correction Factor will lead to a higher $\operatorname{Var}\left(\beta_{1}\right)$ for second order $\mathrm{PQL}$. 


\subsection{Optimal experimental designs for the model with random intercept and random slope}

\subsubsection{Optimal experimental designs for first order MQL with known variance components}

In this section the treatment effect $\beta_{1}$ is assumed to be a randomly varying parameter. This means that there is interaction between treatment and school, and the multilevel Jogistic model becomes

$$
\operatorname{logit} \pi_{i j}=\ln \left(\frac{\pi_{i j}}{1-\pi_{i j}}\right)=\beta_{0}+\beta_{1} x_{i j}+u_{0 j}+u_{1 j} x_{i j}
$$

where $u_{0 j}$ and $u_{1 j}$ are random effects at the school level, and so corresponding to the intercept and the slope, respectively. These random effects have zero mean and variances $\sigma_{u 0}^{2}$ and $\sigma_{u 1}^{2}$ respectively, and covariance $\sigma_{u \mid 11}$. For randomization at the pupil level, there are two treatment conditions per school and both $\sigma_{u 0}^{2}$ and $\sigma_{u 1}^{2}$ can be estimated. There is only one treatment condition per school for randomization at the school level so $\sigma_{u 0}^{2}$ and $\sigma_{u 1}^{2}$ cannot be disentangled. In fact their sum is estimated which will be denoted by $\sigma_{u}^{2}$ in this section.

For first order MQL with known variance components the $\operatorname{Var}\left(\beta_{1}\right)$ and the optimal allocation of units, together with the $\operatorname{Var}\left(\beta_{1}\right)$ for the optimal allocation of units is given in Table 6.5 (see the appendix for the derivation for the $\operatorname{Var}\left(\hat{\beta}_{1}\right)$ ). This table, of course, shows that the pupil level is still the optimal level of randomization, but the $\operatorname{Var}\left(\hat{\beta}_{1}\right)$ is higher than that for models without school by treatment interaction and so the ratio of the $\operatorname{Var}\left(\beta_{1}\right)$ for randomization at the pupil level and the $\operatorname{Var}\left(\hat{\beta}_{1}\right)$ for randomization at the school level is higher than that for models without school by treatment interaction. Furthermore, for both levels of randomization the optimal allocation of units depends on the true parameter values which of course have to be known or for which a reasonable guess must be available. Again, a lower $\operatorname{Var}\left(\hat{\beta}_{1}\right)$ can be obtained by using a larger budget $C$, which results in sampling more schools. As in Section 6.3 the results are equal to those for linear multilevel models as given in Chapter 3 when $\sigma_{e}^{2}$ is replaced by $\delta^{2}=\frac{1}{2}\left(4+e^{\beta_{0}+\beta_{1}}+e^{\beta_{0}-\beta_{1}}+e^{-\beta_{0}+\beta_{1}}+e^{-\beta_{0}-\beta_{1}}\right)$, and the same substitution may be applied to the results for models with three levels as well. 
Table 6.5 Var( $\left.\hat{\beta}_{1}\right)$, Optimall Allocation of Units, and $\operatorname{Var}\left(\hat{\beta}_{1}\right)$ given Optimal Allocation of Units First Order MQL with Known Variance Components Models with Random Intercepts and Random Slopes.

\begin{tabular}{|c|c|c|c|c|c|}
\hline $\begin{array}{l}\text { Level of } \\
\text { randomization }\end{array}$ & $\operatorname{Var}\left(\beta_{1}\right)$ & \multicolumn{2}{|c|}{ optimal $n_{1}$} & optimal $n_{2}$ & $\operatorname{Var}\left(\beta_{1}\right)$ given optimal $n_{1}, n_{2}$ \\
\hline \multirow{2}{*}{ pupil } & $n_{1} \sigma_{u 1}^{2}+\delta^{2}$ & 8 & $c_{2}$ & $C$ & $\left(\sigma_{u \sqrt{c_{2}}}+\delta_{\sqrt{c_{1}}}\right)^{2}$ \\
\hline & $n_{1} n_{2}$ & $\sigma_{u 1}$ & $\overline{c_{1}}$ & $\frac{\delta}{\sigma_{u 1}} \sqrt{c_{1} c_{2}}+c_{2}$ & $C$ \\
\hline \multirow{2}{*}{ school } & $n_{1} \sigma_{w}^{2}+\delta^{2}$ & 8 & $c_{2}$ & $C$ & $\left(\sigma_{u} \sqrt{c_{2}}+\delta \sqrt{c_{1}}\right)^{2}$ \\
\hline & $n_{1} n_{2}$ & $\sigma_{u}$ & $\overline{c_{1}}$ & $\frac{\delta}{\sigma_{u}} \sqrt{c_{1} c_{2}}+c_{2}$ & $C$ \\
\hline
\end{tabular}

Note. Treatment conditions are coded -1 and $+1 . \sigma_{u}^{2}=\sigma_{u 0}^{2}+\sigma_{u 1}^{2}$ $\delta^{2}=\frac{1}{2}\left(4+e^{\beta_{0}+\beta_{1}}+e^{\beta_{0}-\beta_{1}}+e^{-\beta_{0}+\beta_{1}}+e^{-\beta_{0}-\beta_{1}}\right)$.

\subsubsection{Optimal experimental desigus for $\mathrm{PQL}$ and numerical integration}

For randomization at the pupil level a simulation study was done to calculate the Sampling Variance $\left(\beta_{1}\right)$ and the Correction Factor which is necessary to translate the variance of the treatment effect estimator for first order MQL into that for $\mathrm{PQL}$ or numerical integration. The computer program MLwiN (Goldstein et al., 1998) was used for PQL estimation, the maximum number of iterations was set equal to 100 , and the convergence criterion was set equal to 0.001. MIXOR (Hedeker and Gibbons, 1996) was used for numerical integration. Ten quadrature points, automatic starting values, a convergence criterion of 0.001 , and a maximum of 1000 iterations were used. For small values of the variance components, estimation by numerical integration may produce incorrectly high estimates of the variance components, and only those data sets for which $\hat{\sigma}_{u 0}^{2}<3$ and $\hat{\sigma}_{u 1}^{2}<3$ were used for calculating the Correction Factor.

The parameter values used in the simulation study were $\beta_{0}=0 ; \beta_{1}=0,0.5,1$, or 1.5 ; $\sigma_{w 0}^{2}=0,0.25,0.5$, or $1 ; \sigma_{w 1}^{2}=0$, or 0.25 . The sample sizes were $\left(n_{1}=10, n_{2}=40\right), \quad\left(n_{1}=20\right.$, $\left.n_{2}=20\right)$, and $\left(n_{1}=40, n_{2}=10\right)$. Thus, there were 96 combinations of parameter values and allocations of units.

The results of the simulation study are presented in Table 6.6 , showing that for each of the estimation methods the Sampling Variance $\left(\beta_{1}\right)$ increases with $\sigma_{u 1}^{2}, \beta_{1}$ (given $\beta_{0}=0$ ), and the number of pupils per school, as was also the case for first order MQL with known variance components. The Correction Factor is again close to unity for first order PQL, about 1.2 for second order PQL, and about 1.4 for numerical integration. 
Table 6.6 Sampling Variance $\left(\hat{\beta}_{1}\right)$ (Correction Factor) for $\mathrm{PQL}$ and Numerical Integration Models with Random Intercepts and Random Slopes

\begin{tabular}{|c|c|c|c|c|c|}
\hline $\begin{array}{l}\text { Parameter/ } \\
\text { Allocation }\end{array}$ & $\begin{array}{l}\text { PQL-1 } \\
\text { IGLS }\end{array}$ & $\begin{array}{l}\text { PQL-2 } \\
\text { IGLS }\end{array}$ & $\begin{array}{l}\text { PQL-1 } \\
\text { RIGLS }\end{array}$ & $\begin{array}{l}\text { PQL-2 } \\
\text { RIGLS }\end{array}$ & $\begin{array}{l}\text { Numerical } \\
\text { integration }\end{array}$ \\
\hline \multicolumn{6}{|l|}{$\overrightarrow{\sigma_{u 0}^{2}}$} \\
\hline 1 & $0.021(1.070)$ & $0.025(1.281)$ & $0.021(1.084)$ & $0.026(1.314)$ & $490)$ \\
\hline 0.5 & 0.020( & 0.023 & 0.020 & 0.024 & 0.02 \\
\hline 0.25 & 0.019( & 0.022 & 0.019 & 0.023 & 67) \\
\hline 0 & $0.020(1$ & $0.022(1.102)$ & $0.020(1$. & $0.022(1$ & $.426)$ \\
\hline \multicolumn{6}{|l|}{$\sigma^{2}$} \\
\hline 0.2 & 0.027 & $0.031(1.154)$ & $0.027(0.998)$ & $0.032(1.180)$ & 253) \\
\hline 0 & $0.013(1.044)$ & $0.015(1.193)$ & $0.013 i$ & 0.01601 & $0.019(1.520)$ \\
\hline \multicolumn{6}{|l|}{$\beta_{1}$} \\
\hline 1.5 & 0.025 & 0.031 & 0.02 & 0.032 & 25) \\
\hline 1 & 0.020 & 0.023 & 0.020 & 0.024 & 30) \\
\hline 0.5 & 0.0 & 0.02 & 0.0 & 0.0200 & 91) \\
\hline 0 & 0.0 & $0.019(1.098)$ & $0.017(1.007)$ & $0.019(1.113)$ & $0.022(1.300)$ \\
\hline \multicolumn{6}{|l|}{$n_{1}, n_{2}$} \\
\hline & & I) & 0.0 & 0.028 & \\
\hline 20,20 & $0.019(1.019)$ & $0.022(1.164)$ & $0.019(1.029)$ & $0.023(1.191)$ & $0.026(1.485)$ \\
\hline 10,40 & $0.016(1.011)$ & $0.020(1.240)$ & $0.016(1.018)$ & $0.020(1.265)$ & $0.022(1.428)$ \\
\hline
\end{tabular}

Furthermore, the results of the simulation study show that $\beta_{1}$ was underestimated by at most $10 \%$ of the true $\beta_{1}$ by first order PQL and overestimated by at most $5 \%$ of the true $\beta_{1}$ by second order $P Q L$ and numerical integration. The ratio of the Sampling Variance $\left(\beta_{1}\right)$ and the Mean Variance $\left(\beta_{1}\right)$ was close to unity for first and second order PQL. For numerical integration it may be as large as 5 for $\sigma_{u 0}^{2}=0$, and is generally below unity for $\sigma_{u 0}^{2}>0$.

\subsubsection{An example: planning of a school-based smoking prevention intervention (continued)}

We will now assume that there is a treatment by school interaction in the example from Section 6.3.3, and that a random slope has to be modeled. If the variances of the two random effects have to be estimated separately, then randomization has to be done at the pupil level. If $\beta_{0}=0, \beta_{1}=0.5, \sigma_{u 0}^{2}=0.15, \sigma_{u 1}^{2}=0.10$ then $\delta^{2}=4.255$ and the optimal allocation of units is given by $n_{1}=29.17, n_{2}=4$.07. Rounding off to $n_{1}=30, n_{2}=4$ gives $\operatorname{Var}\left(\beta_{1}\right)=0.060$ and the precondition of not sampling more than 30 pupils per school is satisfied. Multiplication of the $\operatorname{Var}\left(\beta_{1}\right)$ by 1.2 has to be done when second order PQL is used, see Table 6.4. So, assuming treatment by school interaction results in sampling more schools and a higher $\operatorname{Var}\left(\hat{\beta}_{1}\right)$ than when this interaction is assumed absent, but pupil level randomization is still superior to school level randomization. 


\subsection{Power of the test of no treatment effect}

By choosing the optimal level of randomization and optimal sample sizes as given in the previous two sections, the $\operatorname{Var}\left(\beta_{1}\right)$ can be minimized which will lead to maximal power of the test of no treatment effect. So the design issue on power of the test of no treatment effect is related to the design issues of optimal level of randomization and optimal sample sizes. In this section it is shown how the power can be calculated. The null hypothesis of no treatment effect may be tested using the test statistic $z=\hat{\beta}_{1} / \sqrt{\operatorname{Var}\left(\hat{\beta}_{1}\right)}$. The computer programs MIXOR (Hedeker and Gibbons, 1996) and MLWN (Goldstein et al., 1998) both assume that this test statistic has a standard normal distribution when the null hypothesis is true. Given this assumption, for two-sided tests with alternative hypothesis $\mathrm{H}_{\mathrm{a}}: \beta_{1} \neq 0$ and significance level $\alpha$, the power $\gamma$ can be calculated by (e.g., Cochran, 1983)

$$
\sqrt{\operatorname{Var}\left(\beta_{1}\right)}=\frac{|\Delta|}{z_{1-\alpha L / 2}^{+z_{1-\gamma}}}
$$

where $\Delta$ is the minimally rellevant deviation of $\beta_{1}$ from zero, and $z_{1-\alpha / 2}$ and $z_{1-\gamma}$ are the $100(1-\alpha / 2) \%$ and $100(1-\gamma) \%$ standard nomal deviates. For a one sided test $z_{1-\alpha / 2}$ has to be replaced by $z_{1-\alpha}$.

\subsubsection{An example: planning of a school-based smoking prevention intervention (continued)}

The power of the test of no treatment effect for second order PQL is given in Table 6.4 for the allocations of units as calculated in Sections 6.3.3 and 6.4.3. A one-sided alternative hypothesis $\left(\mathrm{H}_{2}: \beta_{1}>0\right)$ is used, the significance level $\alpha$ was taken equal to 0.05 , and the minimally relevant deviation of $\beta_{1}$ from zero was taken to be equal to $\Delta=0.5$. As is always the case, the power is inversely related to $\operatorname{Var}\left(\boldsymbol{\beta}_{1}\right)$. Reasonable power is obtained with randomization at the pupill level in case there is no treatment by school interaction.

\subsection{Discussion and conclusions}

In this study three design issues for experiments with binary outcomes in multilevel populations were dealt with: the optimal level of randomization, the optimal allocation of units given the costs and budget for sampling and measuring units, and the power of the test of no treatment effect given the level of randomization and a budget for measuring and sampling As optimality criterion the variance of the estimator of the regression coefficient of the treatment effect $\operatorname{Var}\left(\beta_{1}\right)$ is used since in experimental designs this regression coefficient is generally of main interest. Moreover, $\operatorname{Var}\left(\beta_{1}\right)$ is inversely related to the power of the test of no treatment effect, so minimum $\operatorname{Var}\left(\beta_{\mathbb{1}}\right)$ leads to maximum power assuming unbiased estimates of the treatment effect. This statistical optimality criterion may or may not conflict 
with other criteria in designing an optimal experiment. More practical criteria consist of political and logistical reasons, the need to reduce costs and administrative efforts, and the need to avoid treatment group contamination, which occurs when information leaks from the intervention to the control group (e.g., Donner, Brown, and Brasher, 1990; Raudenbush, 1997; Donner, 1998). Sometimes there is no altemative to cluster randomization. In community-based interventions, for example, the intervention will necessarily affect all members of a cluster. Ethical criteria for choosing a certain design may also be applied. In some circumstances, it may be unethical to treat certain individuals within a group while others are not treated. In this case randomization at the individual level will become impossible.

The analytical results for first order MQL with known variance components show that the lowest level is the optimal level of randomization. When treatment by school interaction (i.e. a random slope) is assumed to be absent, the optimal allocation of units is globally optimal when randomization is done at the pupil level, since it does not depend on the parameter vallues. A smaller $\operatorname{Var}\left(\beta_{1}\right)$ may be obtained by using a higher budget for sampling and measuring which results in sampling more units at the level at which randomization is done. When treatment by school interaction is present, a higher budget results in sampling more schools for both levels of randomization. The $\operatorname{Var}\left(\hat{\beta}_{1}\right)$ as calculated for first order MQL has to be multiplied by the Correction Factor equal to 1.2 for second order $\mathrm{PQL}$ and equal to 1.2 or 1.4 for numerical integration and a model without or with a random slope, respectively. $\operatorname{The} \operatorname{Var}\left(\beta_{1}\right)$ for first order $\mathrm{PQL}$ is lower than that for second order $\mathrm{PQL}$ or mumerical integration since it equals to that for first order MQL, but more biased estimates are obtained for first order PQL.

In this paper it was assumed that the budget for sampling and measuring is fixed. Given a level of randomization, the optimal allocation of units as well as the $\operatorname{Var}\left(\hat{\beta}_{1}\right)$ can then be established. The last step is then to calculate the power of the test of no treatment effect. Of course, one can also start by specifying the needed power of the test of no treatment effect. From this the $\operatorname{Var}\left(\hat{\beta}_{1}\right)$ as well as the needed budget for sampling and measuring can be calculated, given the level of randomization. Then the last step is to establish the optimal allocation of units.

It must be emphasized that in experiments the treatment effect is often of main interest and that the derivation of the optimal level of randomization and the optimal allocations of units were based on the variance of the treatment effect estimator. If covariates are also included into the model (to increase statistical power or to correct for confounding) the variance of the treatment effect estimator has to be multiplied by the variance inflation factor (VIF) in case of linear and logistic models without random effects. The VIF is equal to $1 /\left(1-R^{2}\right)$ where $R^{2}$ is equal to the squared multiple correlation coefficient between treatment effect and the covariates (Lipsitz and Parzen, 1995; Hsieh, Bloch and Larsen, 1998). For multilevel linear models with two levels of nesting and fixed slopes a similar 
result holds (Moerbeek et al, 1999). For multilevell logistic models more research is necessary. Furthermore, if covariates are included in the model $D$ - optimal and $A$ - optimal designs can be derived as well. Finally, in this study a balanced design and randomization into equal numbers of experimental and control schools and pupils were assumed. It will also be useful to derive optimal designs for more general cases.

\section{References}

Bach, D., Van Den Berg-Segers, A., Hübner, A., Van Breukelen, G., Cesana, M., \&Plétan, Y. (1995). Rufloxacin once daily versus ciprofloxacin twice daily in the treatment of patients with acute uncomplicated pyelonephritis. The Joumal of Urology, 154, 19-24.

Breslow, N. E., \& Clayton, D. G. (1993). Approximate inference in generalized hinear models. Journal of the American Statistical Association, 88, 9-25.

Bryk, A. S., \& Raudenbush, S. W. (1992). Hierarchical linear models. Newbury Park: Sage Publications.

Bryk, A. S., Raudenbush, S. W., \& Congdon, R. T., Jr. (1996). HLM: Hierarchical Linear and Nonlinear Modeling with the HLM/2L and HLM/3L Prograns. Chicago: Scientific Software International.

Cochran, W. G. (1983). Plamning and analysis of observational siudies. New York: Willey

De Vries, H., Backbier, E., Dijkstra, M., Van Breukelen, G., Parcel, G., \& Kok, G. (1994). A Dutch social influence smoking prevention approach for vocational school students. Health Education Research, 9, 365-374.

Donner, A. (1998). Some aspects of the design and analysis of cluster randomization trials. Applied Statistics, 47, 95-113.

Donner, A., Birkett, N., \& Buck, C. (1981). Randomization by cluster. Sample size requirements and analysis. American Journal of Epidemiology, 114, 906-914.

Donner, A., Brown, K. S., \& Brasher, P. (1990). A methodological review of non- therapeutic intervention trials employing cluster randomization, 1979-1989. International Journal of Epidemiology, 19, 795-800.

Feng, Z., \& Grizzle, J. E. (1992). Correlated binomial variates: properties of estimator of intraclass correlation and its effect on sample size calculation. Statistics in Medicine, 11, 1607-1614.

Gibbons, R. D., \& Hedeker, D. (1997). Random effects probit and logistic regression models for three-level data: Biometrics, 53, 1527-1537.

Goldstein, H. (1986). Multilevel mixed linear model analysis using iterative generalized least squares. Biometrika, 73, 43-56.

Goldstein, H. (1989). Restricted unbiased iterative generalized least squares estimation. Biometrika, $76,622-623$.

Goldstein, H. (1991). Nonlinear multilevel models, with an application to discrete response data. Biometrika, 78, 45-51.

Goldstein, H. (1995). Multilevel statistical models, 2nd ed. London: Edward Amold.

Goldstein, H., \& Rasbash, $J$ (1996). Improved approximations for multilevel models with binary responses. Journal of the Royal Siatistical Society" Series A, 159, 505-513.

Goldstein, H, Rasbash, J, Plewis, I, Draper, D., Browne, W., Yang, M., Woodhouse, G., \& Healy, M. (1998). A user"s guide to MLwiN. London: Institute of Education.

Hedeker, D. \& Gibbons, R. D. (1994). A random-effects ordinal regression model for multilevel analysis. Biometrics, 50, 933-944.

Hedeker, D. \& Gibbons, R. D. (1996). MIXOR: a computer program for mixed-effects ordinal regression analysis. Computer Methods and Programs in Biomedicine, 49, 157-176.

Hedeker, D., McMahon, S. D., Jason, L. A., \& Salina, D. (1994). Analysis of clustered data in 
community psychology: with an example from a worksite smoking cessation project. American Joumal of Community Psychology, 22, 595-61.5.

Hox, J. J. (1994), Applied multilevel analysis. Amsterdam: TT-Publikaties.

Hsieh, F. Y. (1988). Sample size formulae for intervention studies with the oluster as unit of randomization. Statistics in Medicine, 8, 1195-1201.

Hsieh, F. Y., Bloch, D. A., \& Larsen, M. D. (1998). A simple method of sample size calculation for linear and logistic regression. Statistics in Medicine, 17, 1623-1634.

Kreft, I., \& De Leeuw, J. (1998). Introducing multilevel modeling. London: Sage Publications.

Lee, E. W., \& Dubin, N. (1994). Estimation and sample size considerations for clustered binary data. Statistics in Medicine, 13, 1241-1252.

Lipsitz, S. R., \& Parzen, M. (1995). Sample size calculations for non-randomized studies. The Statistician, 44, $81-90$.

Liu, G., \& Liang, K.-Y. (1997). Sample size calculations for studies with correlated observations. Biometrics, 53, 937-947.

Longford, N. T. (1995). Random coefficient models. Oxford: Clarendon Press.

Moerbeek, M, Van Breukelen, G. J. P., \& Berger, M. P. F. (in press). Design issues for experiments in multilevel populations. Journal of Educational and Behavioral Statistics.

Maerbeek, M, Van Breukelen, G. J. P., \& Berger, M. P. F. (1999). Optimal experimental designs for linear multilevel models with covariates. Manuscript submitted for publication.

Raudenbush ${ }_{n}$ S. W. (1997) Statistical analysis and optinal design for cluster randomized trials. Psychological Methods, 2, 173-185.

Rodriguez, G., \& Goldman, N. (1995). An assessment of estimation procedures for multilevel models. with binary data. Journal of the Royal Statistical Society, Series A, 158, 73-89.

Shih., W. J. (1997). Sample size and power calculations for periodontal and other studies with clustered samples using the method of generalized estimating equations. Biometrical Journal, 39, 899-908.

Snijders, T. A. B., \& Bosker, R. J. (1993). Standard errors and sample sizes for two-level research. Journal of Educational Statistics, 18, 237-259.

Snijders, T. A. B., \& Bosker, R. J. (1999). Multilevel analysis: An Introduction to Basic and Advanced Multilevel Modeling. London: Sage Publications.

Woolf, B. (1955). On estimating the relation between blood group and disease. Annals of Human Genetics, 19, 251-253.

\section{Appendix}

\section{Calculation of the $\operatorname{Var}\left(\hat{\beta}_{\mathbb{1}}\right)$ for known variance components}

In this appendix $\operatorname{Var}\left(\beta_{1}\right)$ is derived for two levels of nesting (i.e. pupils within schools) and models with interaction between treatment condition and school. The $\operatorname{Var}\left(\beta_{1}\right)$ for models without interaction is obtained by setting $\sigma_{u \mathbb{1}}^{2}=0$. We make use of the fact that for the linearized model, the multilevel model and the aggregated data model give the same results if the variance components are known. In the models below $x_{i j}$ is coded +1 for the intervention group and -1 for the control group. 
If there is interaction between treatment condition and school, we have the transformed model

$$
y_{i j}^{*}=\beta_{0}+\beta_{1} x_{i j}+u_{0 j}+u_{1 j} x_{i j}+e_{i j}^{*}
$$

where $\operatorname{var}\left(\epsilon_{i j}^{*}\right)=\left[\pi_{i j}\left(1-\pi_{i j}\right)\right]^{-1}=2+e^{-\beta_{0}-\beta_{1}}+e^{\beta_{0}+\beta_{1}}$ if $x_{i j}=+1$, and $2+e^{-\beta_{0}+\beta_{1}}+e^{\beta_{0}-\beta_{1}}$ if $x_{i j}=-1$, and assuming $\hat{u}_{0 j}=\hat{u}_{1 j}=0$, since a first order MQL approximation is used.

For pupill level randomization the difference between the average outcomes in the two treatment groups in school $j$ is given by

$$
\left(\bar{y}_{j /}^{*}-\bar{y}_{j k}^{*}\right)=\left(\left(\beta_{0}+\beta_{1}+u_{0 j}+u_{1 j}+\bar{e}_{j k}^{*}\right)-\left(\beta_{0}-\beta_{1}+u_{0 j}-u_{1 j}+\bar{e}_{j i c}^{*}\right)\right)=2 \beta_{1}+2 u_{1 j}+\left(\bar{e}_{j i}^{*}-\bar{e}_{j k}^{*}\right)
$$

where the intervention and control group are denoted by subscripts $t$ and $c$, respectively. This difference has expectation $\beta_{1}$, and so $\beta_{1}$ may be estimated by this difference averaged across the schools:

$$
\beta_{1}=\frac{1}{n_{2}} \sum_{j=1}^{n_{2}} \frac{1}{2}\left(\bar{y}_{j l}^{*}-\bar{y}_{j l}^{*}\right)=\frac{1}{n_{2}} \sum_{j=1}^{n_{2}}\left(\beta_{1}+u_{1 j}+\frac{1}{2}\left(\bar{e}_{j t}^{*}-\bar{e}_{j l}^{*}\right)\right),
$$

which has variance

$$
\begin{aligned}
\operatorname{Var}\left(\hat{\beta}_{1}\right) & =\operatorname{Var}\left(\frac{1}{n_{2}} \sum_{j=1}^{n_{2}} \frac{1}{2}\left(\bar{y}_{j t}^{*}-\bar{y}_{j c}^{*}\right)\right)=\frac{1}{n_{2}^{2}} \sum_{j=1}^{n_{2}} \operatorname{Var}\left(u_{1 j}+\frac{1}{2}\left(\bar{e}_{j t}^{*}-\bar{e}_{j l}^{*}\right)\right) \\
& =\frac{n_{1} \sigma_{i 1}^{2}+\frac{1}{2}\left(4+e^{-\beta_{0}-\beta_{n}}+e^{\beta_{0}+\beta_{1}}+e^{\beta_{i j}-\beta_{1}}+e^{-\beta_{0}+\beta_{1}}\right)}{n_{1} n_{2}} .
\end{aligned}
$$

For school level randomization we have school means

$$
\begin{aligned}
& \bar{y}_{j}^{*}=\beta_{0}+\beta_{1}+u_{0 j}+u_{1 j}+\bar{e}_{j}^{*} \text { if } x_{j}=+1 \\
& \bar{y}_{j}^{*}=\beta_{0}-\beta_{1}+u_{0 j}-u_{1 j}+\bar{e}_{j}^{*} \text { if } x_{j}=-1
\end{aligned}
$$

and $\beta_{1}$ may be estimated by 


$$
\hat{\beta}_{1}=\frac{1}{2}\left(\frac{\sum_{j=1}^{\frac{1}{3} n_{2}} \bar{y}_{j}^{*}}{\frac{1}{2} n_{2}}-\frac{\sum_{j=\frac{1}{2} n_{2}+1}^{n_{2}} \bar{y}_{j}^{*}}{\frac{1}{2} n_{2}}\right)=\beta_{1}+\frac{1}{2}\left(\frac{\sum_{j=1}^{\frac{1}{2} n_{2}} u_{0 j}+u_{1 j}+\bar{e}_{j}}{\frac{1}{2} n_{2}}-\frac{\left.\sum_{j=\frac{1}{2} n_{2}+1}^{n_{2}} u_{0 j}-u_{j j}+\bar{e}_{j j}^{*}\right)}{\frac{1}{2} n_{2}}\right),
$$

if the first $\frac{1}{2} n_{2}$ schools are in the treatment group and the others are in the control group. This statistic has variance

$$
\begin{aligned}
\operatorname{Var}\left(\hat{\beta}_{1}\right) & =\frac{1}{4} \operatorname{Var}\left(\frac{\sum_{j=1}^{\frac{1}{2} n_{2}} \bar{y}_{j}^{*}}{\frac{1}{2} n_{2}}-\frac{\sum_{j=\frac{1}{2} n_{2}+1}^{n_{2}} \bar{y}_{j}^{*}}{\frac{1}{2} n_{2}}\right)=\frac{1}{4} \operatorname{Var}\left(\frac{\sum_{j=1}^{\frac{1}{n_{2}} n_{2}} u_{0 j}+u_{1 j}+\bar{e}_{j}^{*}}{\frac{1}{2} n_{2}}-\frac{\left.\sum_{j=\frac{1}{2} n_{2}+1}^{n_{2}} u_{0 j}-u_{v j}+\bar{e}_{j}^{*}\right)}{\frac{1}{2} n_{2}}\right) \\
& =\frac{1}{4}\left(\frac{\sigma_{u 0}^{2}+\sigma_{u 1}^{2}+2 \sigma_{u 01}+\frac{2+e^{-\beta_{0}-\beta_{1}}+e^{\beta_{0}+\beta_{u 1}}}{n_{1}}}{\frac{1}{2} n_{2}}+\frac{\sigma_{u 0}^{2}+\sigma_{u 1}^{2}-2 \sigma_{u 01}+\frac{2+e^{-\beta_{0}+\beta_{1}}+e^{\beta_{0}-\beta_{1}}}{n_{1}}}{\frac{1}{2} n_{2}}\right) \\
& =\frac{n_{1}\left(\sigma_{u 0}^{2}+\sigma_{u 1}^{2}\right)+\frac{1}{2}\left(4+e^{-\beta_{0}-\beta_{11}}+e^{\beta_{0}+\beta_{1}}+e^{\beta_{0}-\beta_{1}}+e^{-\beta_{0}+\beta_{1}}\right)}{n_{1} n_{2}} .
\end{aligned}
$$




\title{
Chapter 7
}

\section{Optimal sample sizes for health education intervention studies}

\begin{abstract}
This paper deals with optimal sample sizes for health education intervention studies with individuals nested within clusters. As an example a Dutch school-based smoking prevention intervention, with pupils nested within schools, will be considered. Optimal sample sizes will be given for models with continuous or binary outcomes, and for one or two treatment factors. Because pupils are nested within schools $s_{p}$ not only the total number of pupils needed to obtain a specified power of the test of treatment effect has to be established, but also the number of schools and the number of pupils per school. It will be shown that different outcome variables will lead to different optimal sample sizes. Furthermore, the statistically optimal design is not allways feasible in practice because of certain limitations, and it will be shown how to calculate the optimal design for limited sample sizes at both the school and pupil level.
\end{abstract}

Key words: optimal sample sizes, standard errors , power, smoking prevention intervention

We wish to thank Marlein Ausems, Ilse Mesters and Hein De Vries for their permission to use the data set and the cost calculation. Their study is part of an European project, called the Octopus project, which was supported by financial grants from the European Commission (soc96 200568 05FO2/soc 97202491 05F02). 


\subsection{Introduction}

Large-scalle health education interventions, such as school-based smoking prevention interventions involving several schools, generally demand a high amount of time, labor, and money. Furthermore, the willingness of schools, teachers, and pupils to participate in an intervention study which is not part of the standard curriculum is necessary. These efforts would be somewhat wasted if the study were not able to detect an existing treatment effect. It is therefore necessary to plan such intervention studies as optimally as possible, which means to establish the optimal sample sizes at both the school and pupil level in advance. Sample size formulae are available for continuous outcome variables under the assumption that either the number of schools or the number of individuals per school is fixed (Donner, Birkett, and Buck, 1981; Hsieh, 1988; Siddiqui, Hedeker, Flay, and Hu, 1996). It is, however, often more realistic to calculate optimal sample sizes under the restriction that there is a fixed budget for sampling and measuring units, as has been done for continuous outcome variables by Raudenbush (1997), and Snijders and Bosker (1993). This budget may be calculated such that a certain statistical power is achieved for the test of the treatment effect.

There is a problem however. The costs for the enrolment of schools and pupils, as well as the values of the parameters in the statistical model that will be used to analyze the data have to be known in advance in order to calculate the optimal number of schools and pupils per school. This means that intervention studies are developed and implemented to get some knowledge of the parameter values, notably the intervention effect, but these values have to be known in advance in order to plan the study as optimally as possible. To solve this problem a reasonable prior estimate of these parameter values may be used instead of the true parameter values. Such an estimate may be obtained from a pilot study, from results of comparable intervention studies, and from theoretical opinions about the minimally relevant treatment effect.

In this paper the process of the optimal planning of intervention studies will be illustrated with the results obtained from the study by Ausems, Mesters, De Vries, and Van Breukelen (1999), and it will be shown how an optimal design may be found given the study budget. Ausems et al. (1999) investigated the effects of a Dutch school-based smoking prevention intervention. This intervention aimed at preventing and delaying the onset of smoking, and therefore targeted elementary school pupils aged 11 and 12 years old (the highest group of the Dutch elementary schools), since research indicated that the smoking Dutch youth had smoked their first cigarette at that age. The study evaluated the effects of an existing in-school prevention program in which pupils received information on different aspects of smoking (e.g. smoking and health, costs of smoking, advertisements) during lessons. An out-of-school intervention, in which pupils received personalized tailored letters on smoking, was evaluated simultaneously, since research indicated that the in-school intervention would function more effectively when it was supplemented with an out-of-school intervention. So there were two treatment factors with two levels each, which resulted in a total of four treatment groups: a group receiving the in-school intervention ( 34 schools), a group receiving the out-of-school intervention ( 36 schools), a group 
receiving both ( 36 schools), and a control group ( 34 schools). Randomization to treatment groups was done at the school level. A total of 3349 pupils participated in the study, the number of pupils per school ranged from 3 till 53 with a mean of 23.9 , and $79 \%$ of the school sizes ranged between 15 and 35. Questionnaires measured the attitude toward smoking, sociall influence, selfefficacy expectations, intention to smoke, smoking behavior, and social-demographic variables like age and gender at pre- and several post-tests. We will restrict ourselves to onlly two of the outcome measures of the questionnaire at the first post-test to illustrate the results on optimal designs in our paper. For a more extensive data analysis and discussion of the results we refer to the paper by Ausems et al. (1999), and forthcoming papers by the same authors.

The contents of the current paper are as follows. In the next section the data are analyzed to get some knowledge of the values of the parameters. Formula for optimal sample sizes for continuous and binary outcomes will be given in Section 7.3, and it will be shown how the power of detecting a treatment effect can be calculated. The results in this section are based on the papers on optimal designs mentioned above, and on Moerbeek, Van Breukelen, and Berger (in press, 1999a, 1999b). Optimal designs for the study by Ausems et al. (1999) will be derived in Section 7.4 using the parameter estimates from Section 7.2. Optimal designs will be derived for a continuous outcome variable (attitude towards the disadvantages of smoking), and a binary one (smoking behavior).

\subsection{Analysis of the data from the Dutch smoking prevention intervention}

In the Dutch smoking prevention intervention study pupils were nested within schools, and it is reasonable to assume that smoking behavior of pupils within the same schools is correlated due to mutual influence and school policy towards smoking, and similarly for other outcomes such like attitude. This means that the traditional regression model, which ignores nesting of pupils within schools and depiendency of outcomes within a school, will] incorrectly estimate standard errors of predictor variables, and this may lead to incorrect conclusions on the effect of an intervention. The data analysis should take into account the nesting of pupils within schools and the dependency of outcomes within the same school, and this may be done by using the multilevel model (Bryk and Raudenbush, 1992; Goldstein, 1995; Hox, 1994; Kreft and De Leeuw, 1998; Longford, 1995; Snijders and Bosker, 1999). Multilevel models may be used when the schools in the intervention study can be looked upon as a random sample from a population of schools. For a review of multilevel and more traditional models for mested data we refer to Moerbeek, Van Breukelen, and Berger (1999c).

The basic idea of multilevel modeling is that regression models are formulated at each level of the multilevel data structure, and that the models may be combined into one single model. In our example, the pupil level model rellates the outcome ATTITUDE (attitude lowards the disadvantages of smoking at post-test) to relevant cowariates at that level: PRE ATTITUDE (attitude towards the disadvantages of smoking at pre-test), AGE, and GENDER. The model for pupil $i$ within school $j$ is then 
ATTITUDE $_{i j}=\beta_{0 j}+\beta_{1 j}$ PRE_ATTITUDE $_{i j}+\beta_{2 j}$ AGE $_{i j}+\beta_{3 j}$ GENDER $_{i j}+e_{i j}$

The random error terms $e_{i j}$ are independently and normally distributed with zero mean and variance $a_{e}^{x}$. The pre-test measurement on attitude was included into the model to correct for pretest differences in attitude, thereby reducing unexplained variance and increasing statistical power. Age and gender were included since it was thought that these are related with the attitude towards smoking. Multilevel models differ from traditional regression models since they allow each school to have its own intercept $\beta_{0 j}$ and slopes $\beta_{1 j}, \beta_{2 j}$, and $\beta_{3 j}$, which may vary as a function of school level covariates and a random school effect. We will assume that the slopes do not vary (which was confirmed by a preliminary data analysis), and that the intercept varies as a function of the dummy variables associated with the treatment conditions and a random school level term. The school level model is then

$$
\begin{aligned}
& \beta_{0 j}=\beta_{0}+\beta_{4} \mathbb{N}_{j}+\beta_{5} \text { OUT }_{j}+\beta_{6} \mathrm{IN} * \text { OUT } \\
& \beta_{1 j}=\alpha_{1} \\
& \beta_{2 j}=\beta_{2} \\
& \beta_{3 j}=\beta_{3},
\end{aligned}
$$

where $\mathbb{I N}=+1$ for a school participating in the in-school intervention and -1 otherwise, so that this variable is centered around zero and $\beta_{4}$ measures $h a l f$ the effect of the in-school intervention averaged across both levels of the OUT factor. The variable OUT for the out-of-school intervention is coded likewise, and the interaction term $\mathbb{N N}^{*}$ OUT is calculated as the product of the two, and so has values -1 and +1 as well. The random terms $u_{0 j}$ are independently and normally distributed with zero mean and variances $\sigma_{u 0}^{2}$. Moreover, the $u_{0 j}$ are also independently distributed of $e_{i j}$. Substitution of the school level model into the pupil level model gives the single equation model

$$
\begin{gathered}
\text { ATTITUDE }_{i j}=\beta_{0}+\beta_{3} \text { PRE_ATTITUDE } \\
+\beta_{4} \mathrm{IN}_{j}+\beta_{5} \mathrm{\beta}_{2} \mathrm{AGE}_{j}+\beta_{3} \mathrm{OUENDER}_{i j}+\beta_{6} \mathrm{IN} * \mathrm{OUT}_{j}+u_{0 j}+e_{i j^{*}}
\end{gathered}
$$

Table 7.1 shows the coding scheme and the average outcomes for the four treatment groups. As follows from Table $7.1, \beta_{4}$ is equal to half the average difference in outcomes between groups 3 and 4 on the one hand and groups 1 and 2 on the other. Likewise, $\beta_{5}$ is equal to half the average difference in outcomes between groups 2 and 4 on the one hand and groups $\mathbb{1}$ and 3 on the other, and $\beta_{6}$ is equal to half the average difference in outcomes between groups 1 and 4 on the one hand and groups 2 and 3 on the other. The residual or unexplained variance of the outcomes is equal to $\sigma_{u 0}^{2}+\sigma_{a}^{2}$, whereas the residual covariance of outcomes within the same school is equal to $\sigma_{t 0}^{2}$. The intra-school correlation coefficient $\rho$ measures the relative amount of variance at the school level and is calculated as $\rho=\sigma_{w 0}^{2} /\left(\sigma_{w 0}^{2}+\sigma_{e}^{2}\right)$. 
Table 7.1 Coding Scheme and Average Outcomes for the four Intervention Groups

\begin{tabular}{|c|c|c|c|c|c|}
\hline Group & Intervention & $\mathbb{N N}$ & OUT & $\mathrm{IN*}$ OUT & Average outcome \\
\hline 1 & none & -1 & -1 & +1 & $\begin{array}{c}\beta_{0}+\beta_{1} \text { PRE_ATTITUDE }+\beta_{2} \text { AGE }+ \\
\beta_{3} \text { GENDER }-\beta_{4}-\beta_{5}+\beta_{6}\end{array}$ \\
\hline 2 & out-of-school & -1 & +1 & -1 & $\begin{array}{c}\beta_{0}+\beta_{1} \text { PRE_ATTITUDE }+\beta_{2} \text { AGE }+ \\
\beta_{3} \text { GENDER }-\beta_{4}+\beta_{5}-\beta_{6}\end{array}$ \\
\hline 3 & in-school & +1 & -1 & -1 & $\begin{array}{c}\beta_{0}+\beta_{1} \text { PRE_ATTITUDE }+\beta_{2} \text { AGE }+ \\
\beta_{3} \text { GENDER }+\beta_{4}-\beta_{5}-\beta_{6}\end{array}$ \\
\hline 4 & both & +1 & +1 & +1 & $\begin{array}{c}\beta_{0}+\beta_{1} \text { PRE_ATTITUDE }+\beta_{2} \text { AGE }+ \\
\beta_{3} \text { GENDER }+\beta_{4}+\beta_{5}+\beta_{6}\end{array}$ \\
\hline
\end{tabular}

Table 7.2 Predictors of Attitude towards Disadvantages of Smoking

\begin{tabular}{|c|c|c|c|c|}
\hline Variable & Estimate & Standard Error & $\bar{Z}$ & $p$-value \\
\hline Intercept & $\beta_{0}=24.198$ & 2.646 & 9.144 & 0.000 \\
\hline Pre-treatment attitude & $\hat{\beta}_{1}=0.564$ & 0.016 & 35.344 & 0.000 \\
\hline Age & $\hat{\beta}_{2}=-0.610$ & 0.217 & -2.809 & 0.005 \\
\hline \multicolumn{5}{|l|}{ Condition } \\
\hline program & $\hat{\beta}_{4}=0.783$ & 0.198 & 3.963 & 0.000 \\
\hline (OUT) & $\hat{\beta}_{5}=1.198$ & 0.197 & 6.070 & 0.000 \\
\hline interaction (IN*OUT) & $\hat{\beta}_{6}^{2}=-0.630$ & 0.197 & -3.190 & 0.001 \\
\hline Random school effect & $\hat{\sigma}_{t+0}^{2}=3.351$ & 0.642 & & \\
\hline Random pupil effect & $\partial_{a}^{2}=44.949$ & 1.122 & & \\
\hline
\end{tabular}

Note. $\quad$ Log Likelihood $=-11193.886$

The dummy variable $\mathrm{IN}$ is coded +1 for a pupil receiving the in-school intervention and -1 , otherwise. The dummy variable OUT is coded likewise, and the dummy variable $\mathbb{I N}^{*}$ OUT is the product of the two. 
The computer program MLXREG (Hedeker and Gibbons, 1996) was used for the data analysis. The results of the analysis with the model in (7.3) are given in Table 7.2 , and the reported $p$-walues are two-tailed. The regression coefficient $\beta_{3}$ associated with the variable GENDER was found not significant at the $5 \%$ level and excluded from the model. The results in Table 7.2 show that the post-test attitude increases when the pre-test attitude increases, but decreases when the age increases. Participation in the in-school or out-of-school intervention results in a higher attitude, but participation in both interwentions adds a negative effect to the effects of the in-school and out-of-school interventions. Since the design is almost balanced at the school level and the dummy variables $\mathbb{N}$ and OUT are coded -1 and +1 , these dummy variables are uncorrelated with each other and with the $\mathbb{N N}^{*}$ OUT interaction term. Therefore, $\beta_{4}$ and $\beta_{5}$ may be interpreted as in a model without an interaction term, see Table 7.1. The estimated intra-school correlation is equal to $3.351 /(3.351+44.949)=0.069$ and significantly differs from zero.

The multilevel model for the binary outcome variable SMOKE differs from the linear model in that the dependent variable is equal to $\log \left(\pi_{i j} /\left(1-\pi_{i j}\right)\right)$ where $\pi_{i j}$ is the probability of being a smoker (i.e. SMOKE $=1$ ):

$$
\begin{aligned}
\log \left(\frac{\pi_{i j}}{1-\pi_{i j}}\right) & =\beta_{0}+\beta_{1} \text { PRE_SMOKE }_{i j}+\beta_{2} \mathrm{AGE}_{i j}+\beta_{3} \mathrm{GENDER}_{i j} \\
& +\beta_{4} \mathrm{IN}+\beta_{5} \mathrm{OUT}_{j}+\beta_{6} \mathrm{IN}^{*} \mathrm{OUT}_{j}+u_{0 j}
\end{aligned}
$$

where PRE SMOKE is the smoking behavior at pre-test. The results of the data analysis with the second order Penalized Quasi Likelihood estimator (which is available in the computer program MLwiN by Goldstein et al., 1998) for the dependent variable SMOKE are given in Table 7.3, and the reported $p$-values are two-tailed. The variables GENDER and IN*OUT were excluded from the model since their effects were found non-significant. Although the variable IN was non-significant, it was maintained in the model since it is one of the variables of primary interest. Participation in the out-of-school intervention leads to a lower probability of being a smoker at post-test. A higher age or being a smoker at pre-test also leads to a higher probability of being a smoker at post-test. In Table 7.3 the results from the model where either $\mathbb{N}$ or OUT is the only explanatory variable are also given since these are necessary for calculating the optimal design as is shown in Section 7.3. 
Table 7.3 Predictors of Smoking Behavior

\begin{tabular}{|c|c|c|c|c|}
\hline Variable & Estimate & Standard Enror & $\overline{\mathrm{Z}}$ & $p$-value \\
\hline \multicolumn{5}{|c|}{ All predictor variables } \\
\hline Constant & $\hat{B}_{0}=-9.639$ & 1.557 & -6.190 & 0.000 \\
\hline Pre-treatment smoking behavior & $\beta_{1}=2.922$ & 0.166 & 17.628 & 0.000 \\
\hline Age & $\hat{\beta}_{3}=0.556$ & 0.132 & 4.219 & 0.000 \\
\hline \multicolumn{5}{|l|}{ Condition } \\
\hline program $(\mathbb{N})$ & $\hat{\beta}_{4}=-0.042$ & 0.102 & -0.408 & 0.683 \\
\hline tailor (OUT) & $\beta_{5}=-0.241$ & 0.102 & -2.362 & 0.018 \\
\hline Random school effect & $\hat{\sigma}_{z 0}^{\frac{3}{2}}=0.580$ & 0.163 & & \\
\hline \multicolumn{5}{|c|}{$\mathbb{N}$ is only predictor variable } \\
\hline Constant & $\beta_{0}=-2.637$ & 0.102 & -25.726 & 0.000 \\
\hline \multicolumn{5}{|l|}{ Condition } \\
\hline program $(\mathbb{N})$ & $\beta_{4}=-0.022$ & 0.102 & -0.213 & \\
\hline Random school effect & $\hat{\sigma}_{w 0}^{2}=0.683$ & 0.167 & & \\
\hline \multicolumn{5}{|c|}{ OUT is only predictor variable } \\
\hline Constant & $\hat{\beta}_{0}=-2.639$ & 0.102 & -25.884 & 0.000 \\
\hline $\begin{array}{l}\text { Condition } \\
\text { tailor }\end{array}$ & & & & \\
\hline $\begin{array}{c}\text { tailor (OUT) } \\
\text { Random school effect }\end{array}$ & $\begin{array}{l}\hat{\beta}_{5}=-0.247 \\
\hat{\sigma}_{w 0}^{2}=0.662\end{array}$ & $\begin{array}{l}0.102 \\
0.165\end{array}$ & -2.427 & \\
\hline
\end{tabular}

Note. The dummy variable $\mathrm{IN}$ is coded +1 for a pupil receiving the in-school intervention and -1 , otherwise. The dummy variable OUT is coded likewise.

\subsection{Optimal sample sizes}

Crucial to the planning of a design is the selection of a model. For the school-based smoking prevention intervention in this study the model given by (7.3), without the covariate GENDER, will be used, and the optimal design for this model will be described in this section. Furthermore, an optimality criterion is needed to construct the optimal design. Several statistical optimality criteria are available, and different optimality criteria may lead to different designs. In the school-based smoking prevention intervention the treatment effect estimators $\beta_{4}, \beta_{5}$, and $\beta_{6}$ are of primary interest, and should be estimated with minimal standard error, since this will lead to smallest confidence intervals for these regression coefficients and to highest power of the test for no treatment effect. These standard errors may be calculated as a function of the number of schools $\left(n_{2}\right)$, the number of pupils per school $\left(n_{1}\right)$ and the variances $\sigma_{u 0}^{2}$ and $\sigma_{e}^{2}$, or the intraschool correlation coefficient $\rho$ :

$$
\operatorname{SE}\left(\hat{\beta}_{4}\right)=\operatorname{SE}\left(\hat{\beta}_{5}\right)=\operatorname{SE}\left(\hat{\beta}_{6}\right)=\sqrt{\frac{n_{1} \sigma_{u t}^{2}+\sigma_{e}^{2}}{n_{1} n_{2}}}=\sqrt{\frac{\left(\sigma_{u 0}^{2}+\sigma_{e}^{2}\right)\left[\left(n_{1}-1\right) \rho+1\right]}{n_{1} n_{2}}} .
$$


Formula (7.5) holds when the dummy variables $\mathrm{N}$, OUT, and $\mathrm{NN}^{*} \mathrm{OUT}$ (which are coded -1 and +1) are uncorrelated with each other, with the interaction term, and with covariates. This may be achieved by using equal numbers of schools per treatment group and equal numbers of pupils per school, and using large sample sizes or pre-stratification on the covariates. When the number of schools per treatment group or the number of pupils per school vary, (7.5) only holds approximately. As follows from (7.5) a larger intra-school correlation leads to larger standard errors, and this occurs even for small intra-school correlation since the intra-school correlation coefficient is multiplied by the number of pupils per school minus one. Traditional formulas do not take into account intra-school correlation and lead to too small standard errors and consequently overestimation of the actual power and a higher type I error rate.

Given the total sample size $n_{1} n_{2}$ the standard errors in (7.5) are minimized by taking the number of pupils per school $n_{1}$ as small as possible and the number of schools $n_{2}$ as large as possible. The number of schools, however, is limited, since enrolling more schools in the intervention study will result in higher costs, and these costs may not exceed the budget that is available for the study. Furthermore, the budget is not only spent on enrolling schools, but also on the participation of pupils in the intervention study. It is therefore necessary to derive the optimal number of schools $\left(n_{2}\right)$, and the optimal number of pupils per school $\left(n_{1}\right)$ such that the cosits for the intervention study will not exceed the maximum budget $C$ that is available for sampling and measuring. The costs may be calculated by multiplying the total number of pupils $n_{1} n_{2}$ by the costs $c_{1}$ per pupil, and adding the total costs at the school level, which is calculated by multiplying the number of schools $n_{2}$ by the costs $c_{2}$ per school. We then have the following pre-condition for minimizing the standard errors in (7.5):

$$
c_{1} n_{1} n_{2}+c_{2} n_{2} \leq C
$$

The optimal sample sizes can then be shown to be equal to:

$$
n_{1}=\sqrt{\frac{\sigma_{c}^{2} c_{2}}{\sigma_{u 0}^{2} c_{1}}}=\sqrt{\frac{(1-\rho) c_{2}}{\rho c_{1}}}, \text { and } n_{2}=\frac{c}{c_{2}+\sqrt{\frac{\sigma_{e}^{2}}{\sigma_{* 0}^{2} c_{1} c_{2}}}}=\frac{c}{c_{2}+\sqrt{\frac{1-\rho}{\rho} c_{1} c_{2}}}
$$

and the standard errors given the optimal sample sizes in (7.7) are equal to:

$$
\operatorname{SE}\left(\beta_{4}\right)=\operatorname{SE}\left(\beta_{5}\right)=\operatorname{SE}\left(\beta_{6}\right)=\frac{\sqrt{\sigma_{u 0}^{2} c_{2}}+\sqrt{\sigma_{e}^{2} c_{1}}}{\sqrt{C}}
$$

See Moerbeek et al. (in press). It follows from (7.7) and (7.8) that a higher budget $C$ leads to enrolling a higher number of schools, $n_{2}$, in the study and to lower standard errors, whereas the 
optimal number of pupils per school, $n_{1}$, does not depend on the budget. In general the optimal sample sizes calculated from (7.7) are real values and have to be rounded off to integer values such that the budget is not exceeded, and the number of schools in this study has to be a multiple of four since there are four treatment groups which have to consist of equal numbers of schools. Rounding off to integer values may lead to a somewhat higher standard error than calculated from (7.8).

In some studies a limited number of possible schools may be available for the intervention study. Then, it can be shown that the optimal number of schools as given by (7.7) should be used if this limited number is larger than the optimal number of schools, and the optimal number of pupils per school and standard error can be calculated from (7.7) and (7.8), respectively. The limited number of schools should be used if this number is smaller than the optimal number of schools given by (7.7), and then the optimal number of pupils per school and the standard error can be calculated from (7.6) and (7.5), respectively. A similar method can be applied when the number of pupils per school is bounded to a maximum.

Optimall designs for multilevel models with binary responses, one treatment factor and no covariates are given by Moerbeek et al. (1999b), and it was shown that for estimation with second order Penalized Quasi Likelihood estimation the optimal samples sizes are equal to those for the linear case as given in (7.7) with $\sigma_{e}^{2}$ replaced by the following term: $\delta^{2}=\frac{1}{2}\left(4+e^{\beta_{0}+\beta_{4}}+e^{\beta_{0}-\beta_{4}}+e^{-\beta_{0}+\beta_{4}}+e^{-\beta_{0}-\beta_{4}}\right)$, which can be shown to be equal to at least four. Here $\beta_{0}$ is the intercept and $\beta_{4}$ is the regression coefficient associated with the dummy variable $\mathbb{I N}$, and their values, as well as the value of $\sigma_{u 0}^{2}$, have to be known to calculate the optimal design. Remember that for the linear multilevel model only the values of the variance components were needed to calculate the optimal design. For the dummy variable OUT, $\beta_{4}$ has to be replaced by $\beta_{5}$. The study by Moerbeek et al. (1999b) showed that for the best present estimation method for binary responses, i.e. second order Penalized Quasi Likelihood, (7.5) and (7.8) must be multiplied by a factor $\sqrt{1.2}$ and that $\sigma_{e}^{2}$ has to be replaced by $\delta^{2}$. The formula for $\delta^{2}$ is more complicated for models with two treatment conditions and covariates, but the optimal design may then be approximated by using parameter estimates from the model in which the variable of interest (IN or OUT) is the only explanatory variable. This approximation has already been shown to work well when this variable is uncorrelated with the other explanatory variables and when the variance $\sigma_{u 0}^{2}$ is equal to zero (Hsieh, Bloch, and Larsen, 1998).

Until now the budget was given and the optimal sample sizes and standard error were calculated. It is, however, also possible to start with a maximum allowable standard error, since the standard error determines the power of the test of the treatment effect and the width of the confidence interval for the treatment effect. The necessary budget can then be calculated from (7.8) and the optimal sample sizes follow from (7.7).

Once the optimal sample sizes and corresponding standard error have been calculated, the power $\gamma$ on the test of no treatment effect for two sided tests can be calculated from 


$$
\operatorname{SE}\left(\hat{\beta}_{4}\right)=\operatorname{SE}\left(\hat{\beta}_{5}\right)=\operatorname{SE}\left(\hat{\beta}_{6}\right)=\frac{|\Delta|}{z_{1-\infty / 2}+z_{1-\gamma}} .
$$

Here $\alpha$ is the significance level of the test, $\Delta$ is the minimal relevant deviation of $\beta_{4}, \beta_{5}$, or $\beta_{6}$ from zero, and $z_{1-\alpha / 2}$ and $z_{1-\gamma}$ are the $100(1-\alpha / 2) \%$ and $100(1-\gamma) \%$ standard normal deviates. For a one sided test $z_{1-\alpha}$, of course, has to be replaced by $z_{1-\alpha .}$. In calculating the power it is assumed that the test statistic for the treatment effect has a standard normal distribution under the null hypothesis, and this assumption seems valid for continuous outcomes and also for binary outcomes if second order Penalized Quasi Likelihood is used for the data analysis (Moerbeek, Van Breukelen, and Berger, 1999d).

\subsection{Optimal sample sizes for the Dutch smoking prevention intervention}

The following costs were calculated for the smoking prevention intervention by Ausems et al. (1999). The costs are given in Dutch guilders, which are denoted $f$. Two Dutch guilders is approximately equal to one US dollar. The costs for a pupil in the control group were $f 4.25$, the additional costs for a pupil who received the tailor intervention were $f 11.5$. Since the optimal design is calculated such that half of the pupils receive the out-of-school intervention, the mean costs at the pupil level may be used for calculating the optimal design and these mean costs are calculated as $c_{1}=f 4.25+\frac{1}{2}(f 11.5)=f 10$. The costs for a school in the control group were equal to $f 94$, the additional costs for a school that participated in the out-of-school intervention were $f 2$, those for a school in the in-school intervention were $f 334$, and the mean costs were $c_{2}=f 94+\frac{1}{2}(f 2+f 334)=f 262$. The total costs of the intervention study were

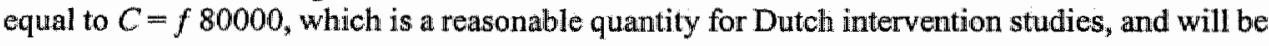
used as budget $C$ for calculating the optimal design. Figure 7.1 shows the maximum number of pupils per school that can be enrolled in the study as a function of the number of schools as was calcuiated from (7.6) using the given costs and budget. As is obvious the number of pupils per school decreases if the number of schools increases.

The cost calculation is based on an initial sample of 160 schools and an average of 25 pupils per school. The questionnaire was completed at both pre- and post-test by 140 schools with an average of 23.9 pupils per school, and so the drop-out rate was $12.5 \%$ at the school level and $4 \%$ at the pupil level. Although data on the dropped-out schools and pupils are not available at both pre- and post- test, they do demand part of the budget and therefore this drop-out has to be taken into account when calculating optimal designs. 


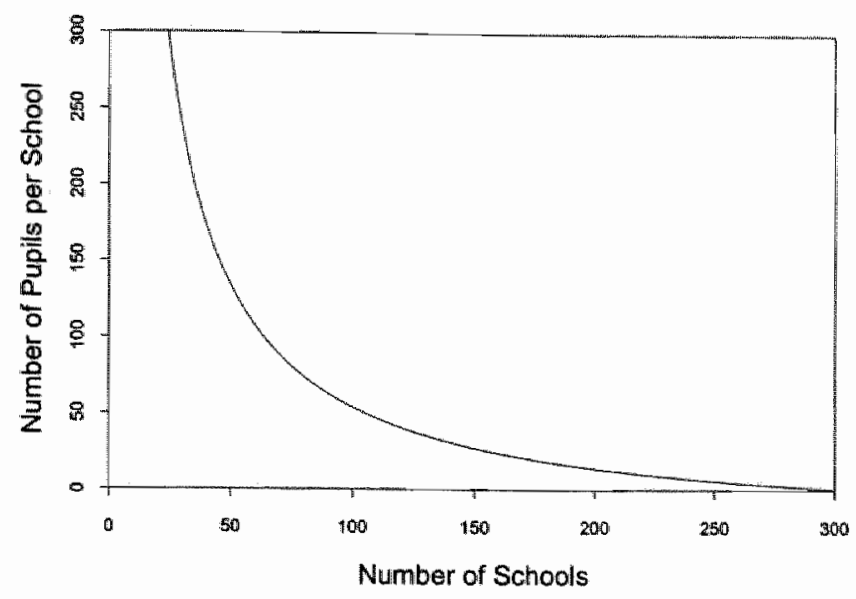

Figure 7.l Number of pupils per school as a function of the number of schools, given $C$ and formula (7.6).

\subsubsection{Optimal sample sizes for the outcome variable attitude}

The optimal design for the outcomes variable ATTITUDE was calculated for $\sigma_{u 0}^{2}=3.351, \sigma_{e}^{2}=44.949, c_{1}=f 10, c_{2}=f 262, C=f 80000$. The optimal designs for the inschool intervention, the out-of-school intervention, and the interaction effect are equal because there are equal numbers of schools in each treatment group and the true (population) values of the regression coefficients $\beta_{4}, \beta_{5}$, and $\beta_{6}$ are not necessary for calculating the optimal design, see (7.5) and (7.8). In calculating the optimal design we used the fact that the dummy variables are uncorrelated with each other and the covariates, due to the $(-1,+1)$ dummy coding scheme that was used and the randomization procedure. For details see Sections 7.2 and 7.3 .

Figure 7.2 shows the standard error of the treatment effects as a function of the number of pupils per school (above) and the number of schools (below), given the budget $C$ and the restriction on $n_{1}$ and $n_{2}$ as given by (7.6) and shown in Figure 7.1. As follows from this plot the standard error hardly changes if the number of schools varies between 150 and 200 . The optimal sample sizes can be calculated from (7.7): $n_{1}=18.7$ and $n_{2}=178.0$. Rounding off to integer values, such that the budget is not exceeded, and taking $n_{2}$ a multiple of four (since there are four treatment groups which have to consist of equal numbers of schools) results in $n_{1}=19, n_{2}=176$. Due to the drop-out the $n_{1}$ and $n_{2}$ decrease by $4 \%$ and $12.5 \%$, respectively, and the standard error increases to 0.194. See Design 1 in Table 7.4.

Design 2 is the design which has $n_{2}=160$ schools, as in the study by Ausems et al. (1999). The standard error for Design 2 is almost equal to that for the optimal design since the number of schools of both designs are between 150 and 200, which is the flat part in the lower 
plot of Figure 7.2 .

The number of schools for Design 1 and 2 is very large. Suppose that only $n_{2}=40$ schools are willing to participate in the intervention study. Then according to (7.6) and Figure $7.1, n_{1}=173$ pupils per school can be enrolled and the standard error will be equal to 0.322 if the drop-out is taken into account (see Design 3a), which is substantially larger than for Designs 1 and 2 . This number of pupils per school, however, is unrealistically high since the intervention aims at 11 and 12 year old pupils and such a large number of pupils is seldom avallable in Dutch elementary schools. Design $3 \mathrm{~b}$ is a design for which $n_{1}=25$ pupils per school and $n_{2}=40$ schools are available and the standard error is equal to 0.386 , which is twice as large as for the optimal design. The corresponding budget is of course smaller.

Design $4 \mathrm{a}$ and $4 \mathrm{~b}$ are the designs for which the number of schools is even lower $\left(n_{2}=20\right)$, and the available number of pupils per school is unlimited or limited to 25 , respectively. It can be seen that the standard errors of these designs are substantially higher than those for Designs 1,2 , and 3 .

Design 5 is the optimal design if one aims at a standard error equal to 0.217 , taking into account the drop-out. Then the costs for the schools and pupils that do not drop-out is equal to $f 54872$ as calculated from (7.8), and $n_{1}=18.7$ and $n_{2}=122.1$ as follows from (7.7). Due to the drop-out rate of $4 \%$ at the pupil level the needed number of pupils per school is equal to $n_{1}=18.7 /(1-0.04)=19.47$, and due to the drop-out rate of $12.5 \%$ at the school level the needed number of schools is equal to $n_{2}=121.5 /(1-0.125)=139.54$. Rounding off to integers, so that $n_{2}$ is a multiple of four, gives $n_{1}=19$ and $n_{2}=140$. The total costs are then equal to $19 \times 140 \times f 10+140 \times f 262=f 63280$. In Figure 7.3 the power for two-sided tests with significance level $\alpha=0.05$ is plotted as a function of the treatment effect. Remember that the treatment effect is equal to twice the value of the regression coefficients $\beta_{4}, \beta_{5}$, or $\beta_{6}$ due to the fact that treatment conditions are coded -1 and +1 . Figure 7.3 shows that almost equal power is obtained by Design 1 and 2, and a somewhat lower power by the less expensive Design 5 . The powers for Designs 3 and 4, however, are much lower. 
Table 7.4 Sample Sizes and Standard Error for Several Designs

\begin{tabular}{llllll}
\hline \hline Design Requirements & \multicolumn{1}{c}{$n_{1}$} & $n_{2}$ & Costs & Stand \\
\hline \multicolumn{5}{c}{ Linear multilevel model } & with outcome variable ATTITU \\
1. & Optimal & 19 & 176 & $f 79552$ & 0.194 \\
2. $\quad n_{2}=160$ & 23 & 160 & $f 78720$ & 0.196 \\
3a. $\quad n_{2}=40$ & 173 & 40 & $f 79680$ & 0.322 \\
3b. $\quad n_{2}=40, n_{1}=25$ & 25 & 40 & $f 20480$ & 0.386 \\
4a. $\quad n_{2}=20$ & 373 & 20 & $f 79840$ & 0.446 \\
4b. $\quad n_{2}=20, n_{1}=25$ & 25 & 20 & $f 10240$ & 0.546 \\
5. & $\mathrm{SE}=0.217$ & 19 & 140 & $f 63280$ & 0.218
\end{tabular}

Multilevel logistic model with outcome variable SMOKE and predictor variable IN

1. Optimal

22

164

f $79048 \quad 0.110$

2. $n_{2}=160$

23

160

f $78720 \quad 0.110$

3a. $n_{2}=40$

173

40

$f 79680$

0.163

3b. $n_{2}=40, n_{1}=25$

25

4a. $n_{2}=20$

40

f 20480

0.215

4b. $n_{2}=20, n_{1}=25$

25

20

f 79840

0.223

5. $\mathrm{SE}=0.123$

26

20

$f 10240 \quad 0.304$

Note. The standard errors are calculated for a drop-out rate of $4 \%$ at the pupil level and $12.5 \%$ at the school level. The dummy variable $\mathrm{IN}$ is coded +1 for a pupil receiving the inschool intervention and -1 , otherwise. The dummy variable OUT is coded likewise, and the dummy variable $\mathbb{N}^{*}$ OUT is the product of the two. 

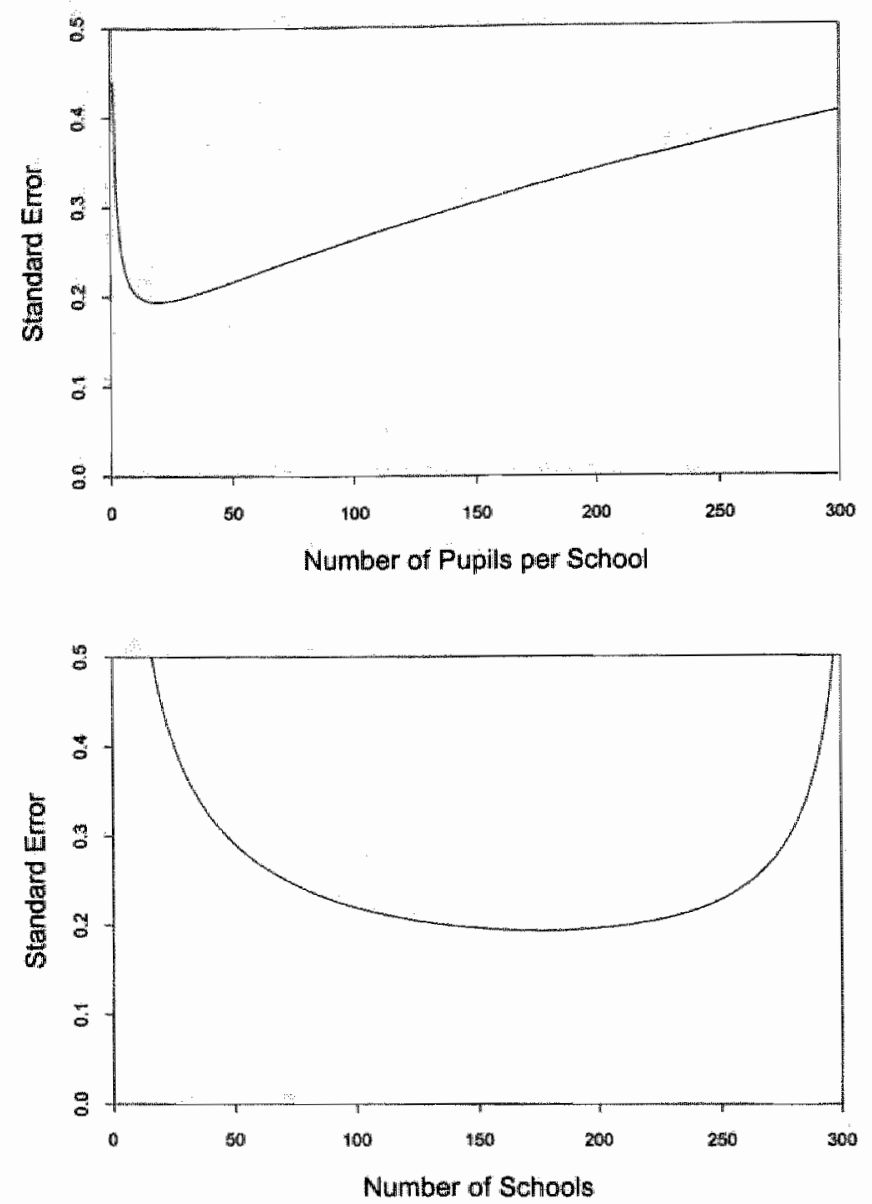

Figure 7.2 Standard error of the treatment effect as a function of the number of pupils per schools (top), and the number of schools (bottom) for the continuous outcome variable ATTITUDE, given the restriction in Figure 7.1. 


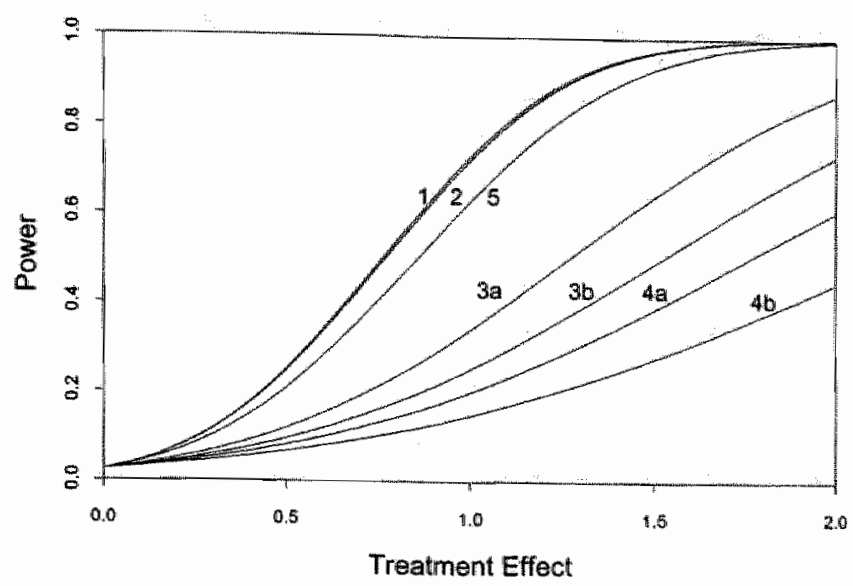

Figure 7.3 Power of two-sided tests as a function of the treatment effect for the designs of Table 7.4 for the continuous outcome variable ATTITUDE. The designs are indicated by their numbers.

\subsubsection{Optimal sample sizes for the outcome variable smoking behavior}

To calculate optimal designs for the outcome variable SMOKE and the dummy variable $\mathrm{N}$, which is associated with the in-school intervention, the multilevel logistic model was fitted with this dummy variable as the only explanatory variable, because sample size formulas have not yet been derived for multilevell logistic models with covariates. Using second order Penalized Quasi Likelihood estimation the following parameter estimates were obtained: $\beta_{0}=-2.637$, $\beta_{1}=-0.044, \delta^{2}=16.042$, and $\sigma_{w 0}^{2}=0.683$, see Table 7.3. The standard error is plotted as a function of the number of pupils per school and the number of schools in Figure 7.4. The standard error hardly changes if the number of schools varies between 125 and 175. The optimal design for integer values of the number of pupils per school and the number of pupils per school gives $n_{1}=22, n_{2}=164$, see Table 7.4. Note that this optimal design differs from that for the continuous outcome variable ATTITUDE, but for both outcome variables the plot of the standard error as a function of the number of schools is very flat when the number of schools is near 160 and designs that yield almost minimal standard error for both outcome variables are therefore available.

The standard error abtained with the optimal design, Design 1, is equal to that of Design 2 which has $n_{2}=160$ schools, as in the study by Ausems et al, (1999). The standard error for Design 2 is equal to that for the optimal design since the number of schools of both designs are between 125 and 175 which is the flat part in the lower plot of Figure 7.4. The standard error for the study by Ausems et al, (1999) as given in Table 7.4 is somewhat swaller than for the optimal 
design since this optimal design was calculated with the use of an approximative method, which may somewhat underestimate or overestimate the standard error, for details see Moerbeek, Van Breukelen, and Berger (1999b).

The standard errors for Design 3 and 4 , which were also used for the continuous outcome variable ATTITUDE, are of course higher than those for Designs 1 and 2.

Suppose the standard error hass to be equal to 0.123 , then the costs for the schools and pupils that do not drop out are equal to $f 53795$, as calculated from (7.8), and the optimal sample sizes are $n_{1}=24.8$ and $n_{2}=105.5$ see (7.7). Due to a drop-out rate of $4 \%$ at the pupil level the needed number of pupils is equal to $24.8 /(1-0.04)=25.8$ which may be rounded off to 26 . The drop-out rate at the school level is $12.5 \%$, so the needed number of schools is $105.5 /(1-0.125)$ $=120.5$ which is rounded off to 120 . The total costs are then $26 \times 120 \times f 10+120 \times f 262=$ $f$ 62640. This is Design 5 in Table 7.4. Figure 7.5 gives the power of two-sided tests for the designs given in Table 7.4 as a function of the treatment effect. Again, remember that the treatment effect is equal to twice the value of the regression coefficients $\beta_{4}$ due to the fact that the predictor variable IN is coded -1 and +1 . The power of the optimal design is equal to that of Design 2. A reasonable power is obtained with the less expensive Design 5. The power for Designs 3 and 4 is much lower.

Finally, it should be noted that for the dummy variable OUT, which is associated with the out-of-school intervention the results are almost equal to that for the dummy variable $\mathrm{IN}$ and not given here. 

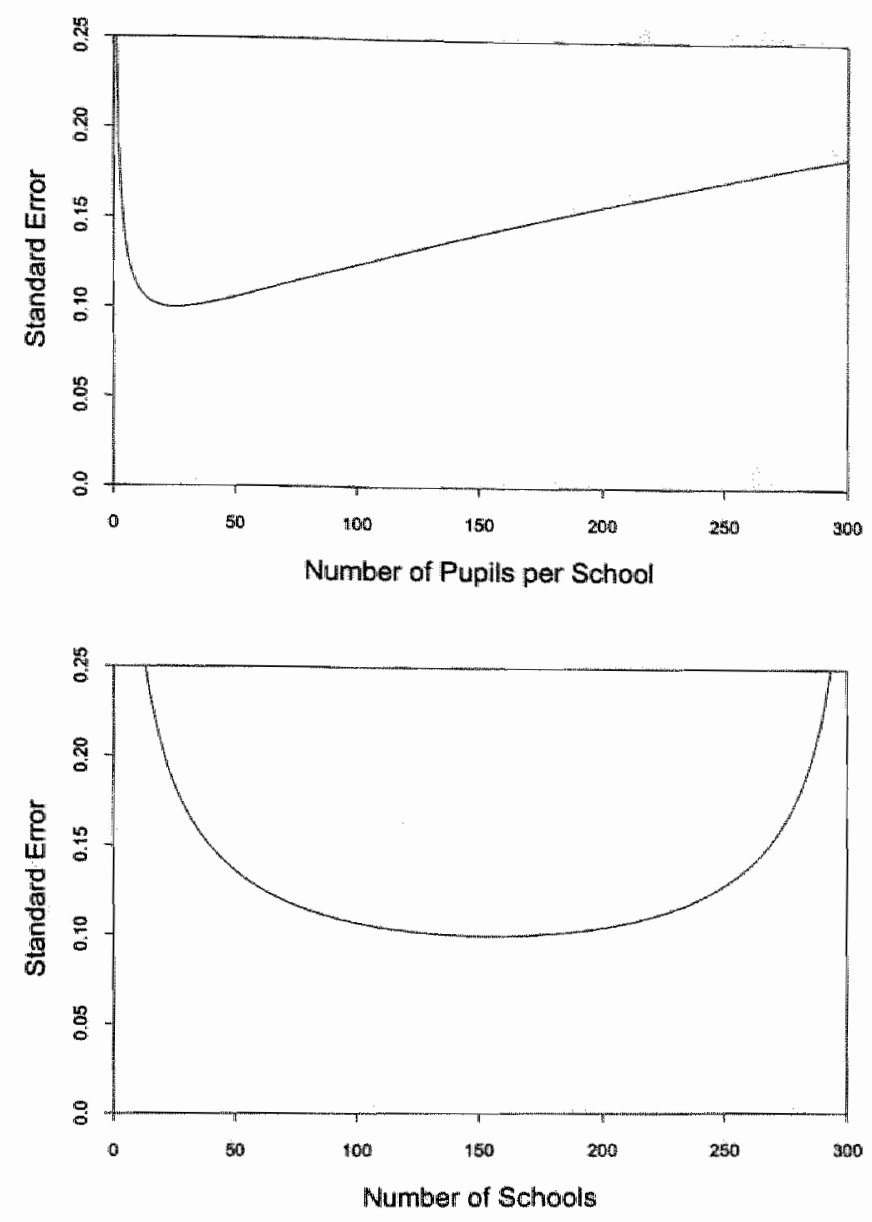

Figure 7.4 Standard error of the treatment effect as a function of the number of pupils per schools (top), and the number of schools (bottom) for the binary outcome variable SMOKE, given the restriction in Figure 7.1. 


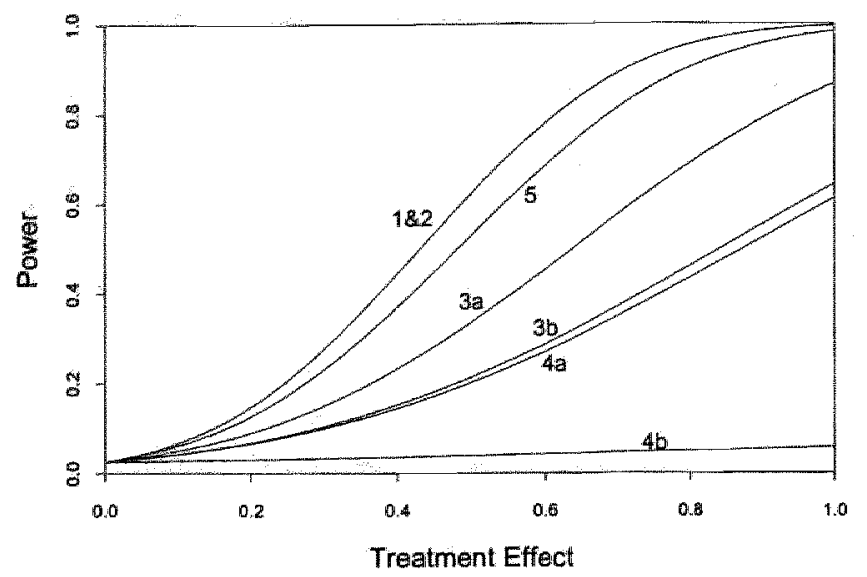

Figure 7.5 Power of two-sided tests as a function of the treatment effect for the designs of Table 7.4 for the binary outcome variable SMOKE. The designs are indicated by their numbers.

\subsection{Discussion and conclusions}

This paper gives optimal sample sizes for health education intervention studies, but the results may also be used for other studies, for instance multi-center clinical trials. Optimal sample sizes are given for models with two levels of nesting, continuous or binary outcomes and two treatment factors for the case where randomization to treatment groups was done at the cluster level. A crucial step in planning an intervention study is the selection of the statistical model for the data analysis. The linear multilevel model can be used for continuous outcomes, whereas binary outcome variables can be analyzed with the multilevel logistic model. Relevant covariates must be selected beforehand and included into the model as well.

A next step is to obtain reasonable prior estimates of the model parameters, the costs at both levels and the budget, and the drop-out rates at both the pupil and school level. These estimates may be obtained from a pilot study, from similar intervention studies, or from theoretical opinions about the minimally relevant treatment effect. Note that for linear multilevel models only the variances at the pupil and school level have to be known to calculate the optimal design, whereas for multilevel logistic models a prior estimate of the regression coefficients has to be given as well. The final step is to calculate the optimal sample sizes at both levels and the corresponding standard error which in turn determines the power on the test of no treatment effect. The optimal sample sizes may be restricted by the actual number of schools and number of pupils per school and these restrictions may be used as pre-conditions when calculating the sample sizes. Furthermore, the standard error will increase due to drop-out at both the pupil and 
school-level, and so drop-out rates should be taken into account when planning the experimental design and budget. It also has to be noted that different outcome variables may lead to different optimal designs, so one should establish the optimal design for the outcome variable of primary interest.

It is also possible to start with a specified power given a minimal relevant deviation from zero of the treatment effect. The standard error of the treatment effect then follows from (7.9), and the needed budget and sample sizes can be calculated from (7.8) and (7.7), respectively. This approach may lead to an almost optimal design with considerably lower costs, and should be considered when planning intervention studies.

The optimal designs presented in this paper are for randomization at the school level. As follows from Moerbeek et al. (in press, 1999a, 1999b) a more efficient design can be achieved by randomization at the pupil level, especially when the intra-school correlation and the number of pupils per school are large. In the school-based smoking prevention intervention, which was used as an example in this paper, this was not possible because the in-school intervention consisted of lessons on different aspects of smoking and had to be delivered class-wise. The outof-school intervention, which consisted of personalized tailored letters, was delivered at the school level as well to avoid treatment group contamination. In other studies, however, randomization at the person level is feasible, for example in double-blind multi-center clinical trials. Formulas for calculating the optimal sample sizes at both levels, given person randomization, are very similar to those presented in this paper and can be found in Moerbeek et al. (in press, 1999a, 1999b).

\section{References}

Ausems, M., Mesters, I., de Vries, H., \& Van Breukelen, G. (1999). Short terms effects of in- and out-ofschool smoking prevention interventions aimed at Dutch elementary schoolchildren. Manuscript in preparation. Maastricht Uniwersity. Department of Health Education and Health Promotion.

Bryk, A. S. \& Raudenbush, S. W. (1992). Hierarchical linear models. Sage Publications, Newbury Park.

Donner, A., Birkett, N., \& Buck, C. (1981). Randomization by cluster. Sample size requirements and analysis. American Joumal of Epidemiology, 114, 906-914.

Goldstein, H. (1995). Multilevel statistical models (2nd ed.). Edward Arnold, London.

Goldstein, H., Rasbash, J., Plewis, I., Draper, D., Browne, W., Yang, M., Woodhouse, G., \& Healy, M. (1998). A user's guide to MLwiN. London: Institute of Education.

Hedeker, D., \& Gibbons, R. D. (1996). MLXREG: a computer program for mixed-effects regression analysis with autocorrelated errors. Computer Methods and Programs in Bionedicine, 49,229 . 252.

Hox, J. J. (1994). Applied multilevel analysis. TT-Publikaties, Amsterdam.

Hsieh, F. Y. (1988). Sample size formulae for intervention studies with the cluster as unit of randomization. Statistics in Medicine, 8, 1195-1201.

Hsieh, F., Bloch, D., \& Larsen, M. (1998). A simple method of sample size calculation for linear and logistic regression. Statistics in Medicine, 17, 1623-1634.

Kreft, I., \& De Leeuw, J. (1998). Intraducing multilevel modelling. Sage Publications, London.

Longford, N. T. (1995). Random coefficient models. Clarendon Press, Oxford. 
Moerbeek, M. Van Breukelen, G. J. P., \& Berger, M. P.F. (in press). Design issues for experiments in multilevel populations. Journal of Educational and Behawioral Statistics,

Moerbeek, M., Van Breukelen, G. J. P., \& Berger, M. P. F. (1999a) Optimal experimental designs for multilevel models with cowariates. Mamuscript submitted for publication. Maastricht University.

Moerbeek, M., Van Breukelen, G. J. P., \& Berger, M. P. F. (1999b). Optimal experimental designs for multilevel logistic models. Manuscript submitted for publication. Maastricht University.

Moerbeek, M. Van Breukelen, G. J.P., \& Berger, M.P.F. (1999c). A review of four different estimation merhods for the analysis of multi-centre trials. Manuscript submitted for publication. Maastricht University.

Moerbeek, M., Van Breukelen, G. J. P., \& Berger, M. P. F. (1999d). A review of estimation methods for multilevel logistic models. Manuscript in submitted for publication. Maastricht University.

Raudenbush, S. W. (1997) Statistical analysis and optimal design for cluster randomized trials. Psychological Methods, 2, 173-185.

Siddiqui, O., Hedeker, D., Flay, B. R., \& Hu, F. B. (1996). Intraclass correlation estimates in a schoolbased smoking prevention study, American Journal of Epidemiology, 144, 425-433.

Snijders, T. A. B., \& Bosker, R. J. (1993). Standard errors and sample sizes for two-level research. Journal of Educational Statistics, 18, 237-259.

Snijders, T. A. B., \& Bosker, R. J. (1999). Multilevel analysis. An introduction to basic and advanced multilevel modelling. Sage Publications, London. 


\section{Epilogue}

Since the 1980 s much attention has been paid to the analysis of multilevel data (i.e. data structures with individuals nested within clusters where the clusters in the sample represent a random sample from a larger population of clusters) in the medical, educational, behavioral and social sciences. This resulted in the development of estimation methods for the multilevel model, and their implementation in computer programs. Although research mainly focused on the analysis of observational data, the multilevel model may be used for experimental data as well. This thesis provides guidelines for the design of experiments when a multilevel data structure is involved, and reviews and compares methods for the analysis of data obtained from such experiments.

Results obtained with the multilevel model may differ from those obtained with more traditional regression models like the fixed effects model, the disaggregated data model and the aggregated data model, and this already happens for quite low intra-cluster correlation coefficients, say $\rho=0.05$. It is therefore necessary to promote the use of multilevel analysis in medical, educational, behavioral, and social studies with a nested design when the clusters in the study are a random sample from a large number of clusters to which the results of the study have to be generalized, which in turn calls for the development of user-friendly computer programs. Special attention has to be paid to the improvement of estimation methods for binary and categorical outcomes since the current estimation methods do not always perform well in terms of parameter convergence of the estimation procedure, parameter estimates, and the distribution under the null hypothesis of the test statistics.

Previous research on sample size calculations for multilevel intervention studies were mainly restricted to two levels of nesting and cluster randomization, and it was mostly assumed that either the number of clusters or the number of individuals per eluster is fixed. These results were extended in this thesis by considering both two and three levels of nesting and randomization at either level of the data structure. Linear and logistic models, with or without cluster by treatment interaction were considered. A cost function, which considered both the costs at the individual and cluster level, was used as a pre-condition. Optimal sample sizes obtained with the formulae in this thesis differ from those obtained with the sample size formulae for the two sample $t$-test for quantitative outcomes or the $\chi^{2}$ contingency test for binary outcomes ignoring the nested data structure, especially when the intra-cluster correlation is high. Since 
incorrectly calculated optimal sample sizes may result in too low power for the test of no treatment effect, it is necessary to promote the use of correct formulae to researchers in the design phase of their studies.

It is expected that in the near future referees of scientific journals will demand that authors of papers use the multilevel model for the analysis of their nested data. Moreover, the rising costs of experiments demand that scientists design their experiments as efficiently as possible. The classical formulae for optimal sample size calculations do not apply to multilevel data, and the formulae presented in this thesis are more appropriate for this purpose. The use of these formulae will lead to more efforts in the design phase, because the costs and budget of the experiment; as well as the values of several parameters in the multilevel model and the minimal relevant treatment effect that needs to be detected have to be specified beforehand. These efforts, however, are repaid since a carefully designed experiment leads to higher statistical power and more efficient use of time, money and labor.

In this thesis, optimal sample sizes were calculated for 50:50 randomization to both treatment groups so that the mean of the costs in both treatment groups may be used in the cost function. Future research may focus on unequal sample sizes in both treatment groups and more complicated cost functions. Also, more research needs to be done on the effect of covariates and unequal cluster sizes on the optimal sample sizes. 


\section{Summary}

In this thesis it was shown that the use of the multilevel model is necessary for the design and analysis of experiments when individuals are nested in clusters and the results from the study have to be generalizable to a population of clusters. Examples of such experiments are schoolbased smoking prevention interventions with pupils nested within schools, and multi-center clinical trials with patients nested within clinics. The use of traditional statistical techniques may lead to incorrect results of statistical tests of and confidence intervals for treatment effects, and to non-optimally designed experiments. Multilevel models are, however, more complicated than more traditional regression models and specialized software is necessary for data analysis when using multilevel models. Therefore a comparison was made in Chapter 2 between the multilevel model and three traditional models for continuous outcomes to show when and how these traditional models fail. The traditional models are the disaggregated data model, the aggregated data model, and the fixed effects model, which considers the clusters (i.e. schools, clinics) to be fixed. The results obtained with the fixed effects model only apply to the clusters in the study. The multilevel model, however, assumes that the clusters represent a random sample from some population, and so the resuits may be generalized to this population. The comparison in Chapter 2 was made for two levels of nesting (pupils within classes), continuous outcomes, and three different situations: randomization at the class level, and randomization at the pupil level with a treatment effect that is constant or that varies across classes, which correspond to a multilevel model with a fixed or random slope, respectively. Criteria for comparison were the estimator of the treatment effect and its variance. The results in this chapter show that traditional models generally lead to an under- or overestimation of the variance of the treatment effect estimator, which may result in type I or type II errors, respectively. Such errors especially occur when the dependency of outcomes within a class and/or the number of pupils per class is large. For balanced designs with a continuous outcome and no covariates several altematives to the multilevel model are available: (a) the fixed effects model may be used when randomization is done at the pupil level and the treatment effect does not vary across classes, (b) the aggregated data model may be used when randomization is done at the pupil level and the treatment effect varies across classes, (c) or when randomization is done at the class level. When covariates are included in the model or the design is unbalanced, however, the multillevel model is 
recommended as analysis model.

Two methods for the analysis of multilevel binary data were compared in Chapter 5: estimation by numerical integration, and Penalized Quasi Likelihood (PQL). $\mathrm{PQL}$ is based on linearization of the non-linear model using a Taylor series expansion, and appears in a first and a second order. The comparison was made for two levels of nesting, models without covariates, and the three situations that were also considered in Chapter 2. The methods were compared on the basis of their point estimator of the treatment effect, and the distribution of the test statistic for testing the treatment effect. The results show that first order PQL performs best in terms of Mean Squared Error of the point estimator of the treatment effect, although there is not much difference with the other methods. For this method, however, the distribution of the test statistic for testing the treatment effect does not have a standard normal distribution under the null hypothesis due to bias in the point estimator. The standard normal distribution may be used as a reference distribution under the null hypothesis for second order PQL. One may choose to use likelihood ratio tests instead of Wald tests when estimation by numerical integration is used. The latter estimation method at least as implemented in the current version of MIXOR has the disadvantage that non-convergence may occur quite often, or that the estimation process may converge to an evidently incorrect estimate. Both second order PQL and estimation by numerical integration perform well in terms of estimating the treatment effect since the relative bias is $5 \%$ or less. The bias may, however, be much higher for variance components estimation, especially with numerical integration with MIXOR, and therefore the development of other estimation methods for multilevel logistic models is necessary.

Chapters 3,4 , and 6 deal with the optimal design of experiments in a multilevel setting, for example school-based smoking prevention interventions with pupils nested within schools. When designing such experiments the optimal level of randomization, and the optimal sample sizes at each level of the data structure have to be established. This not only means determining the total number of pupils, but also the number of schools and the number of pupils per school. The number of schools and the number of pupils per school are restricted by financial limitations since the costs for enrolling schools and pupils may not exceed the budget. The costs are calculated as the sum of the total costs at the school level, which are equal to the costs per school multiplied by the number of schools; and the total costs at the pupil level, which are equal to the costs at the pupil level multiplied by the number of pupils. This means that the number of pupils per school decreases when the number of schools in the experiment increases and vice versa, and the optimal sample sizes at both levels have to be established. Furthermore the optimal sample sizes may be restricted by the actual number of schools or the actual number of pupils per school.

An optimality criterion is necessary for calculating the optimal design. Generally the treatment effect is the parameter of most interest in experiments, and assuming (almost) unbiased point estimation, the variance of its estimator has to be minimized to obtain smallest confidence intervals for the treatment effect and maximal power of the test on treatment effect. This optimality criterion, together with the precondition that the costs may not exceed the budget, 
were used in Chapters 3 and 6 , in which optimal designs were established for linear and logistic multilevel models with two or three levels of nesting. The results in these chapters show that the pupil level is the optimal level of randomization, and randomization at this level is especially preferable when the variance components corresponding to the intercept at higher levells are relatively large and/or when the number of pupils per school is very large. The optimal sample sizes and the variance of the treatment effect estimator given the optimal sample sizes were derived, and of course this variance decreases when the budget increases. The optimal sample sizes at higher levels are equal to two when randomization is done at the pupil level and the treatment effect is constant across higher level units. One might wonder if such a small number of units in a random sample may give enough information about their population. A rule of thumb for selecting between the multilevel and fixed effect model is therefore necessary. Furthermore one may wonder if the variance components at higher levels can be estimated well with such small sample sizes, but these estimated variance components, however, are not necessary for calculating the variance of the treatment effect estimator. This problem, however, generally does not occur when the model contains a randiom slope since then the optimal numbers of higher level units are generally larger than two. The optimal design for multilevel logistic models can only be derived analytically for first order Marginal Quasi Likelihood (MQL), which is known to produce biased estimates. Therefore a simulation study was performed to 'translate' the analytical results on optimal designs obtained with first order MQL into results for PQL estimation or estimation by numerical integration.

Chapter 4 builds upon the results of Chapter 3 by including relevant covariates into the model, a multi-center clinical trial with patients nested within clinics was used as an example. In this chapter not only the variance of the treatment effect estimator was used as optimality criterion, $D$ - and $A$ - optimal designs were derived as well. For these three optimality criteria the patient level was again the optimal level of randomization. The optimal sample sizes did not depend on the optimality criterion for randomization at the clinic level. Furthermore, the effect of including or excluding covariates on the variance components and optimal designs was established in this chapter as well. It was shown that the variance of the treatment effect estimator generally increases when covariates are excluded from the model, at least assuming that the treatment factor is uncorrelated with the covariates due to the randomization procedure.

Chapter 7 illustrates the results on optimal designs from Chapters 3,4, and 6 . In this chapter a school-based smoking prevention intervention was optimally planned using results from a recent study. To plan the intervention as efficient as possible, the costs at both the pupil and school level and the budget have to be known in advance, as well as the drop-out rate at both levels, and the values of the model parameters. This leads to the following problem: intervention studies are developed and implemented to gain some knowledge about the values of model parameters, in particular the treatment effect, but the values of these parameters have to be known to plan an intervention study optimally. To solve this problem a prior estimate may be used, which may be obtained from theoretical knowledge about the smallest relevant treatment 
effect, a pilot study, or a similar intervention study in which the same outcome variables were analyzed. In Chapter 7 several designs were compared and it was shown how to deal with limited school sizes and a limited number of schools. It was shown that an almost optinal design could be achieved with a considerably lower budget.

It has to be noted that this thesis mainly focused on the design and analysis of multilevel experimental data with one treatment factor with two levels. Future research may focus on designing optimal experimental designs for models with more than one treatment factor which may be randomized at either level, for models with one treatment factor with more than two levels, or for models with quantitative treatments. It is also necessary to develop optimal designs for multilevel logistic models with covariates. 


\section{Samenvatting in het Nederlands (Dutch summary)}

Uit dit proefschrift volgt dat het gebruik van het multi-niveau model noodzakelijk is voor het ontwerp en analyse van experimenten waarbij indiwiduen genesteld zijn binnen clusters en wanneer de resultaten van het experiment generaliseerbaar moeten zijn naar een populatie van clusters. Voorbeeldien van zulke experimenten zijn rook-preventie interventies met leerlingen genesteld binnen scholen, en multi-centre klimisch onderzoek met patiënten genesteld binnen klinieken. Het gebruik van traditionele statistische technieken kan leiden tot incorrecte resultaten van statistische toetsen op en betrouwbaarheidsintervallen voor het behandelingseffect, en tot niet-optimaal ontworpen experimenten. Multi-niveau modellen zijn echter complexer dan meer traditionele regressie modellen en specialistische computerprogrammatuur is nodig voor de data analyse wanneer het multi-niveau model gebruikt wordt. Daarom werd in Hoofdstuk 2 een vergelijking gemaakt tussen het multi-niveau model en drie traditionele modellen om aan te tonen wanneer en hoe de traditionele modellen tot incorrecte resultaten leiden. De traditionele modellen zijn het aggregatie model, het disaggregatie model, en het vaste effecten model, welke de clusters (bijvoorbeeld scholen of klinieken) als vaste effecten verondersteld. De resultaten van het vaste effecten model zijn alleen geldig voor die clusters die in het experiment betrokken zijn. Het multi-niveau model beschouwd de clusters echter als een toevallige steekproef uit een populatie, en de resultaten van het onderzoek mogen dan gegeneraliseerd worden naar deze populatie. De vergelijking in Hoofstuk 2 werd gemalkt voor twee niveaus van nesteling (leerlingen in klassen), continue uitkomsten, en drie verschillende situaties: randomisatie naar behandelingscondities op het klas niveau, randomisatie op het leerling niveau met een behandelingseffect dat constant is of dat varieert over de klassen. Dit correspondeert met een multi-niveau model met een vast respectievelijk een random hellingshoek voor het behandelingseffect. Criteria voor de vergelijking waren de schatter van het behandelingseffect en de variantie van deze schatter. De resultaten in dit hoofdstuk laten zien dat traditionele modellen in het algemeen tot een onder- of overschatting van deze variantie leiden. Dit kan resulteren in type I, respectievelijk type II fouten, en dit soort fouten komt vooral voor wanneer de afhankelijkheid van witkomsten binnen eenzelfde klas en/of wanneer het aantall leerlingen per klas groot is. Voor gebalanceerde proefopzetten en modellen zonder covariaten zijn verschillende alternatieven voor het multi-niveau model beschikbaar: (a) het vaste effecten model mag gebruikt 
worden wanneer op het leerling niveau gerandomiseerd wordt en het behandelingseffect varieent over klassen, (b) het aggregatie modiel mag gebuikt worden wanneer op het leerling niveau gerandomiseerd wordt en het behandelingseffect varieert over klassen, (c) of wanneer randomisatie is gedaan op het klas niveau. Het multi-niveau wordt echter aangeraden wanneer covariaten zijn opgenomen in het model of wanneer de proefopzet ongebalanceerd is.

Twee methoden voor de analyse van multi-niveau binaire data werden vergeleken in Hoofdstuk 5: schatting door middel van numeriek integratie, en Penalized Quasi Likelihood (PQL). PQL is gebaseerd op linearisatie van het niet-lineaire model door middel wan een Taylor reeks, waarbij een eerste of tweede orde benadering wordt gebruikt. De vergelijking werd gemaakt voor twee niveaus van nesteling, modellen zonder covariaten, en de drie situaties die ook in Hoofdstuk 2 werden behandeld. De methoden werden vergeleken op basis van hun puntschatter van het behandelingseffect, en de verdeling van de toetsingsgrootheid voor de toets op het behandelingseffect. De resultaten laten zien dat eerste orde PQL de beste parameter schattingen geeft aangezien deze methode de kleinste Mean Squared Error oplevert, alhoewel er weinig verschil is met de andere methoden. Voor eerste orde PQL is de toetsingsgrootheid voor het toetsen van het behandelingseffect niet standaard normaal verdeeld onder de nul hypothese, wat een gevolg is van de onzuiverheid in de puntschatter. De standaard normale verdeling mag echter wel gebruikt worden als referentieverdeling onder de nul hypothese voor tweede orde PQL. Men kan ervoor kiezen likelihood ratio toetsen te gebruiken in plaats van Wald toetsen wanneer schatting door middel van numerieke integratie wordt gedaan. Deze methode heeft echter het nadeel dat de schattingsprocedure vaak niet convergeert, of dat convergentie naar een duidelijk incorrecte schatting optreedt. Zowel tweede orde $\mathrm{PQL}$ als schatting via numerieke integratie schatten het behandelingseffect vrij goed aangezien de relatieve onzuiverheid $5 \%$ of minder is. De onzuiverheid kan echter veel hoger zijn voor het schatten van variantiecomponenten, en de ontwikkeling van andere schattingsmethoden voor multi-niveau logistische modellen is daarom noodzakelijk.

De Hoofdstukken 3,4 , en 6 behandelen het optimaal ontwerpen van experimenten in multi-niveau situaties, bijvoorbeeld rook-preventie interventies met leerlingen genesteld binnen scholen. Bij het ontwerpen van dit soort experimenten moeten het optimale niveau van randomisatie, en de optimale steekproefgrootte op elk niveau bepaald worden. Dit betekent dat niet alleen het totaal aantal leerlingen, maar ook het aantal scholen en het aantal leerlingen per school bepaald moet worden. Het aantal scholen en het aantal leerlingen per school wordt beperkt door tinancielle restricties aangezien de kosten voor het opnemen van leerlingen en scholen in het onderzoek het budget dat daarvoor aanwezig is niet mogen overschrijden. De kosten van het onderzoek worden berekend als de som van de totale kosten op het school niveau, welke gelijk zijn aan de kosten per school vermenigvuldigd met het aantal scholen, en de totale kosten op het leerling niveau, welke gelijk zijn aan de kosten per leerling vermenigvuldigd met het aantal leerlingen. Dit betekent dat het aantal leerlingen per school afneemt wanneer het aantal scholen in het experiment toeneemt en vice versa, en de optimale steekproefgroottes op beide niveaus 
moeten bepaald worden. Deze optimale steekproefgroottes kunnen niet groter zijn dan het werkelijke aantal schollen en het aantal leerlingen per school.

Een optimaliteitscriterium is noodzakelijk voor de berekening van de optimale proefopzet. Het behandelingseffect is over het algemeen de parameter waarin men het meest geinteresseerd is, en de variantie van de schatter van deze parameter moet geminimaliseerd worden om zo smal mogelijke betrouwbaarheidsintervallen voor het behandelingseffect te verkrijgen, en een maximaal onderscheidingsvermogen voor de toets op het behandelingseffect. Dit optimaliteitscriterium, in combinatie met de randvoorwaarde dat het budget niet oversohreden mag worden door de kosten, werd gebuikt in Hoofdstukken 3 and 6 , waarin optimale proefopzetten werden afgeleid voor lineaire en logistische multi-niveau modellen met twee of drie niveaus van nesteling. De resultaten in deze hoofdstukken laten zien dat hel leerling niveau het optimale niveau van randomisatie is, en dat randomisatie op dit niveau vooral de voorkeur heeft wanneer de variantiecomponenten op hogere niveaus relatief groot zijn en/of wanneer het aantal leerlingen per school groot is. De optimale steekproefgroottes en de variantie van de schatter van het behandelingseffect onder de optimale steekproefgroottes werden gegeven, en deze variantie neemt vanzelisprekend af wanneer het budget toe neemt. Voor multi-niveau logistische modellen kunnen de optimale steekproefgroottes alleen analytisch afgeleid worden voor eerste orde Marginal Quasi Likelihood (MQL), welke onzuivere schattingen oplevert. Daarom werd een simulatie studie uitgevoerd om de analytische resultaten voor eerste orde MQL te vertalen in resultaten voor $\mathrm{PQL}$ of schattingen via numerieke integratie. De optimale steekproefgroottes op hogere niveaus zijn gelijk aan twee wanneer randomisatie is ged aan op het leerling niveau en het behandelingseffect constant is over hogere orde eenheden. Men kan zich afvragen of zo'n klein aantal eenheden in een toevallige steekproef genoeg informatie geeft over hun populatie. Een vuistregel voor het kiezen tussen het vaste en het random effecten model is daarom noodzakelijk. Tevens kan men zich afvragen of de variantie componenten op hogere niveaus wel goed geschat kunnen worden met zulke kleine steekproefgroottes. Dit probleem doet zich over het algemeen niet voor wanneer het model een random intercept bevat omdat dan de optimale steekproefgroottes op hogere niveaus over het algemeen groter zijn dan twee.

Hoofdstuk 4 bouwt voort op Hoofdstuk 3 door relevante covariaten in het model op te nemen, een multi-center klinisch onderzoek met patiënten genesteld binnen klinieken werd gebruikt als voorbeeld. In dit hoofdstuk werd niet alleen de variantie van de schatter van het behandelingseffect als optimaliteitscriterium gebruikt, $D$ - en $A$ - optimale proefopzetten werden ook afgeleid. Voor deze drie criteria was het patiềnt niveau het optimalle niveau van randomisatie. De optimale steekproefgroottes hingen niet af van het optimaliteitscriterium wanneer randomisatie werd gedaan op het kliniek niveau. Tevens werd het effect van toewoegen of verwijderen van covariaten op optimale proefopzetten bepaald. De variantie van de schatter van het behandelingseffect neemt ower het algemeen toe wanneer covariaten uit het model verwijderd worden, maar in sommige situaties blijft deze variantie constant wanneer covariaten worden verwijderd of toegevoegd. 
Hoofdstuk 7 illustreert de resultaten over optimale proefopzetten uit de Hoofdstukken 3 , 4, en 6. In dit hoofdstuk werd een rook-preventie interventie optimaal gepland waarbij gebruik werd gemaakt van resultaten van een recent onderzoek. Om de interventie zo optimaal mogelijk te kunnen plannen moeten de kosten op zowel het leerling als het school niveau en het budget van te voren bekend zijn, evenals de uitvalpercentages op beide niveaus, en de waarden van de parameters in het model. Dit leidt tot het volgende probleem: interventie studies worden ontwikkeld en uitgevoerd om enig idee te krijgen over de waarden van de model parameters, in het bijzonder het behandelingseffect, maar de waarden van deze parameters moeten van te voren bekend zijn om een unterventie onderzoek zo optimaal mogelijk te kunnen plannen. Om dit probleem op te lossen kan een prior schatting gebruikt worden, welke verkregen kan worden uit theoretische kennis over het kleinste relevante behandelingseffect, een vooronderzoek, of een vergelijkbare interventie studie waarin dezelfde uitkomstmaten werden geanalyseerd. In Hoofdstuk 7 werden verschillende proefopzetten vergeleken en het werd uitgelegd hoe men om moet gaan met beperkte schoolgroottes en een beperkt aantal scholen. Er werd ook getoond dat een vrijwel optimale proefopzet kan worden bereikt men een aanzienlijk lager budget.

Het moet opgemerkt worden dat in dit proefschrift voornamelijk aandacht besteed werd aan multi-niveau experimentele data met één behandelingsfactor met twee niveaus. Toekomstig onderzoek kan zich richten op het ontwerp van optimale proefopzetten voor modellen met meer dan één behandelingsfactor die op elk niveau gerandomiseerd kunnen worden, voor modellen met één behandelingsfactor met meer dan twee niveaus, of voor modellen met kwantitatieve behandelingsfactoren. Het is ook noodzakelijk om optimale proefopzetten te ontwikkelen voor multi-niveau logistische modellen met covariaten. 


\section{Curriculum Vitae}

Mirjam Moerbeek was born on February 13, 1973 in Westervoort, the Netherlands. From 1985 to 1991 she attended pre-university education at the Van Lingen College in Arnhem. Subsequently she started studying Agricultural Systems Sciences at Wageningen Agricultural University and specialized in Biometrics. In 1994 she wrote a master's thesis in mathematical biology, "Insect-pathogen dynamics: Stage-specific susceptibility and insect density dependence". The research for her master's thesis in statistics, "The prediction of the physical structure of polyethylene terephthalate yarns from wavelet coefficients of Ramani spectra", was done during an internship at Akzo-Nobel in Arnhem in 1995. In 1996 she was a teaching assistant in statistics. In the same year she graduated cum laude and started her work as a $\mathrm{Ph} . \mathrm{D}$. student at the department of Methodology and Statistics at Maastricht University. Her Ph.D. thesis is on "The design and analysis of multilevel intervention studies", and most chapters have been submitted for publication. In 2000 she stayed for three months as a visiting scholar at the department of Biostatistics of the University of California at Los Angeles. Since June 2000 she has been employed as a biostatistician at the Laboratory for Health Effects Research at the National Institute of Public Health and the Environment in Bilthoven. 